This item was submitted to Loughborough's Research Repository by the author.

Items in Figshare are protected by copyright, with all rights reserved, unless otherwise indicated.

\title{
Influence of carbohydrate-electrolyte drinks on muscle metabolism and endurance running performance in man
}

PLEASE CITE THE PUBLISHED VERSION

PUBLISHER

(C) O.K. Tsintzas

LICENCE

CC BY-NC-ND 4.0

\section{REPOSITORY RECORD}

Tsintzas, Orestis-Konstantinos. 2019. "Influence of Carbohydrate-electrolyte Drinks on Muscle Metabolism and Endurance Running Performance in Man”. figshare. https://hdl.handle.net/2134/11739. 


\section{Loughborough University}

This item was submitted to Loughborough University as a PhD thesis by the author and is made available in the Institutional Repository

(https://dspace.lboro.ac.uk/) under the following Creative Commons Licence conditions.

\section{cc) creative}

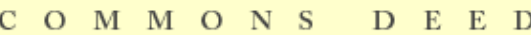

Attribution-NonCommercial-NoDerivs 2.5

You are free:

- to copy, distribute, display, and perform the work

Under the following conditions:

Attribution. You must attribute the work in the manner specified by the author or licensor.

Noncommercial. You may not use this work for commercial purposes.

No Derivative Works. You may not alter, transform, or build upon this work.

- For any reuse or distribution, you must make clear to others the license terms of this work.

- Any of these conditions can be waived if you get permission from the copyright holder.

Your fair use and other rights are in no way affected by the above.

This is a human-readable summary of the Leqal Code (the full license).

Disclaimer 민

For the full text of this licence, please go to: http://creativecommons.org/licenses/by-nc-nd/2.5/ 


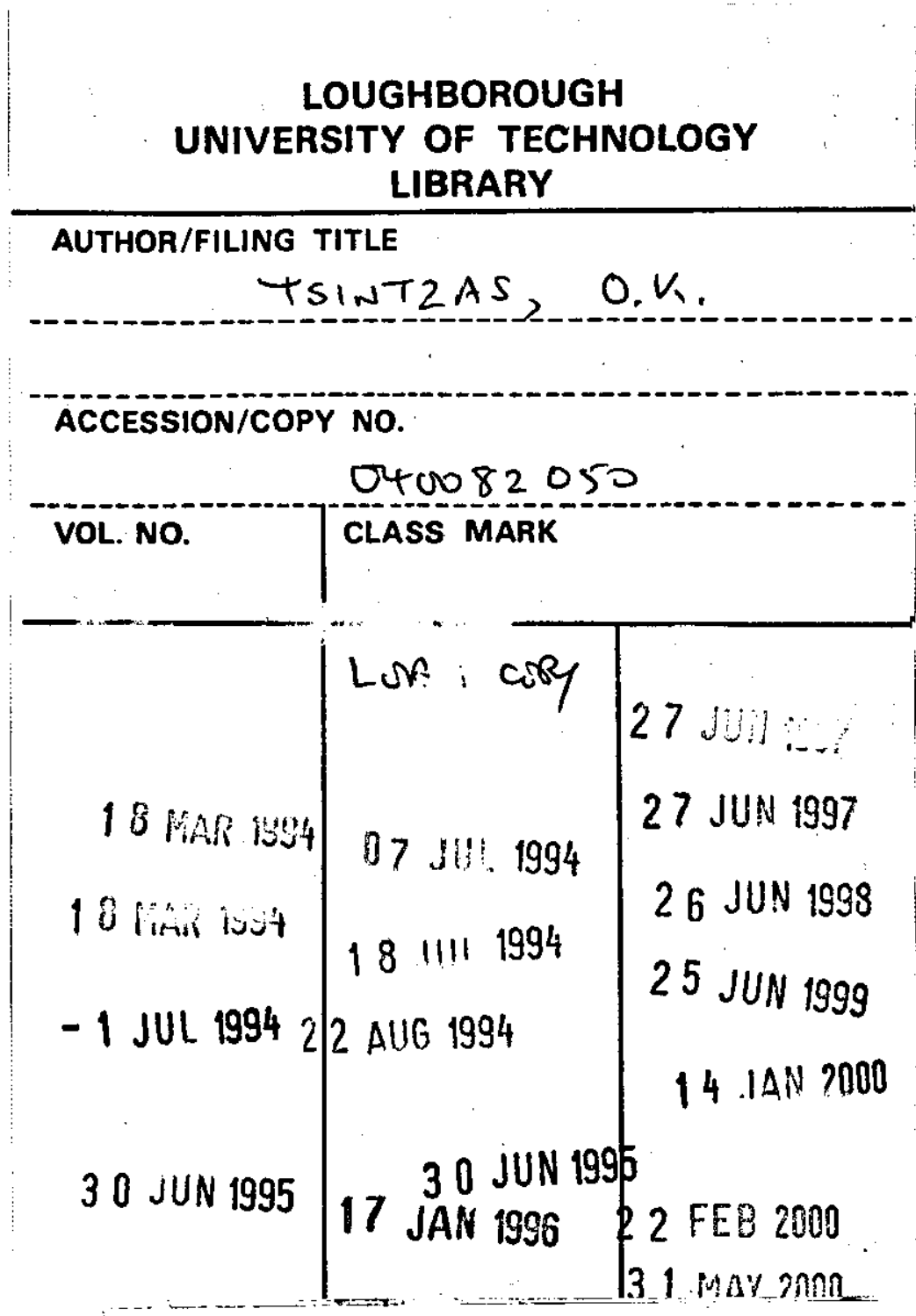

Please note that fines are charged on ALL overdue items.

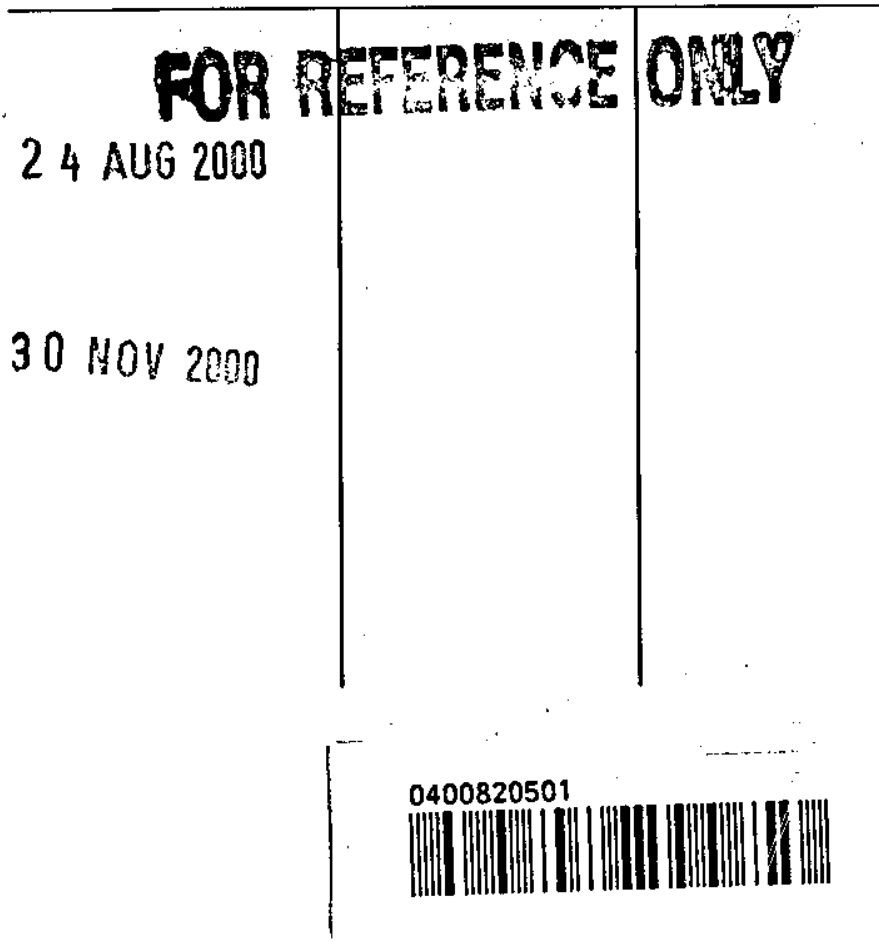





\title{
INFLUENCE OF CARBOHYDRATE-ELECTROLYTE DRINKS ON MUSCLE METABOLISM AND ENDURANCE RUNNING PERFORMANCE IN MAN
}

\author{
by \\ Orestis-Konstantinos Tsintzas
}

\begin{abstract}
A Doctoral Thesis
Submitted in partial fulfilment of the requirements for the award of Doctor of Philosophy of the Loughborough University of Technology
\end{abstract}

October 1993

() by O. K. Tsintzas (1993) 


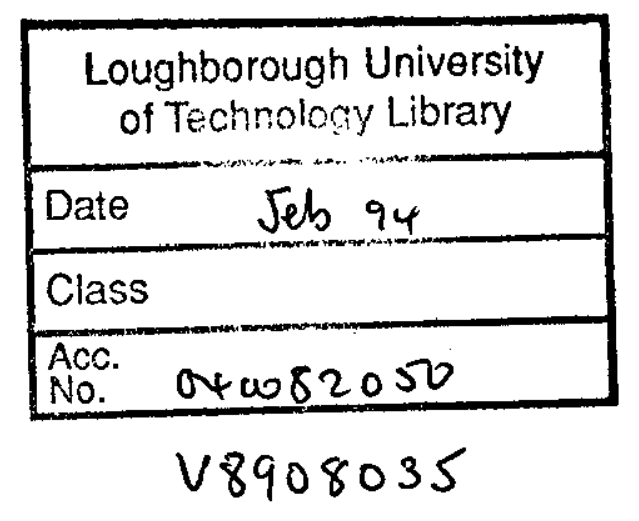




\begin{abstract}
Prolonged running is an activity associated with a wide range of sports and improvement in running endurance is therefore important for those involved. The aims of the first and second studies were to investigate the effect of carbohydrate ingestion on endurance running capacity and performance.
\end{abstract}

In the first study (Chapter 4), seven experienced endurance runners ingested either water $(\mathrm{W})$, a $6.9 \%$ carbohydrate $(\mathrm{CHO})$ solution $(\mathrm{B})$ or a $5.5 \% \mathrm{CHO}$ solution (A) during 3 randomly assigned $42.2-\mathrm{km}$ treadmill runs, 4 weeks apart. Running times for $W, B$ and $A$ trials were $193.9 \pm 5.0 \mathrm{~min}, 192.4 \pm 3.3$ min and $190.0 \pm 3.9 \mathrm{~min}$, respectively. Performance time for the A trial was faster $(p<0.05)$ compared with that of the $W$ trial.

In the second study (Chapter 5), eleven recreational runners completed 3 randomly assigned treadmill runs at $70 \% \mathrm{VO}_{2} \max$ to exhaustion, one week apart. On the first and second occasions a $5.5 \%$ (A) and $6.9 \%$ (C) $\mathrm{CHO}$ solutions were ingested for the first hour of exercise, water was then ingested until exhaustion. On the third occasion water (W) was ingested throughout the run. Performance times for the $W, A$ and $C$ trials were $109.6 \pm 9.6 \mathrm{~min}$, $124.5 \pm 8.4 \mathrm{~min}$ and $121.4 \pm 9.4 \mathrm{~min}$, respectively. Running time to exhaustion for the A trial was significantly longer $(p<0.05)$ compared with the $W$ trial. The results of these studies suggested that the ergogenic effect of the $5.5 \%$ CHO solution was not associated with the prevention of a declining blood glucose concentration and $\mathrm{CHO}$ oxidation rate.

In the third study (Chapter 6), the possibility that sparing of muscle glycogen could be the mechanism by which $\mathrm{CHO}$ ingestion exerts its ergogenic effect was investigated. Seven subjects consumed either a $5.5 \%$ carbohydrate solution (CHO) or water (W) during two 60 -min treadmill runs at $70 \% \mathrm{VO}_{2}$ max a week apart. Biopsy samples from the vastus lateralis muscle were obtained at rest and $60 \mathrm{~min}$ for glycogen determination. A $28 \%$ reduction in glycogen utilization was observed as a result of $\mathrm{CHO}$ ingestion when compared with $W$ ingestion $\left(108.7 \pm 16.3 \mathrm{mmol} . \mathrm{kg}\right.$ dry muscle $(\mathrm{dm})^{-1}$ vs $150.9 \pm 19.9 \mathrm{mmol}_{\mathrm{kg} \mathrm{dm}}-1$ respectively, $\mathrm{p}<0.01$ ). In order to investigate the site of glycogen sparing, individual fibres were dissected and analysed for glycogen. The ingestion of the $\mathrm{CHO}$ solution resulted in sparing of glycogen in Type I (slow twitch) fibres only (38.1\% degradation of glycogen as opposed to $66.2 \%$ during the $W$ trial, $p=0.01$ ).

This glycogen sparing in Type I fibres would only explain the delay in the onset of fatigue observed in the first two studies if glycogen depletion in these fibres was associated with fatigue during prolonged running. In the fourth study (Chapter 7), eight subjects consumed either a 5.5\% carbohydrate solution (CHO) or placebo (PL) during two treadmill runs at $75 \% \mathrm{VO}_{2} \max$ to exhaustion performed one week apart. Biopsy samples from the vastus 
lateralis muscle were obtained at rest and exhaustion in the PL trial and at rest, the point coinciding with PL exhaustion and exhaustion in the $\mathrm{CHO}$ trial. Running times to exhaustion in the PL and CHO trials were $104.3 \pm 8.6$ $\min$ and $132.4 \pm 12.3 \mathrm{~min}(\mathrm{p}<0.01)$. Higher glycogen concentration was observed at the point coinciding with PL exhaustion in the $\mathrm{CHO}$ trial when compared with the value measured at the point of exhaustion in the PL trial (125.6 \pm 22.3 mmol. $\mathrm{kg} \mathrm{d} \mathrm{m}^{-1}$ vs $59.8 \pm 7.9$ mmol. $\mathrm{kg} \mathrm{dm}^{-1} \mathrm{p}<0.05$, respectively). This sparing of muscle glycogen was almost totally restricted to Type I fibres ( $87.1 \pm 18 \mathrm{mmol}^{\mathrm{kg} \mathrm{dm}-1}$ in the CHO trial vs $31.6 \pm 10.3 \mathrm{mmol} . \mathrm{kg}$ $\mathrm{dm}^{-1}$ in the PL trial, $\mathrm{p}<0.01$, respectively). In both the CHO and PL trials, fatigue coincided with glycogen depletion in Type I fibres $(28.1 \pm 7.1 \mathrm{mmol} . \mathrm{kg}$ $\mathrm{dm}^{-1}$ and $31.6 \pm 10.3 \mathrm{mmol}_{\mathrm{kg} \mathrm{dm}}-1$, respectively).

It was suggested, that the spared glycogen became available during the latter stages of the run and, therefore, could account for the improved endurance capacity and performance observed in studies 1,2 and 4 when the $5.5 \% \mathrm{CHO}$ solution was ingested. 
Part of the work on which this thesis is based has been published in the following scientific journals:

Tsintzas, O.K., Williams, C. and Wilson, W. (1993): Influence of carbohydrate ingestion on muscle glycogen utilization during prolonged running in man. J. Physiol. 467: 72P.

Tsintzas, O.K., Williams, C., Boobis, L. and Wilson, W. (1993): Carbohydrate ingestion and endurance running capacity. Int. J. Sports Med. 14 (5): 295. 
This thesis is dedicated to my parents, Maria and Nikos 


\section{ACKNOWLEDGEMENTS}

I would like to give special thanks to the following people for their invaluable help during my $\mathrm{PhD}$ :

My supervisor Professor Clyde Williams for his guidance, understanding and encouragement throughout these years.

My director of research Dr Andrianne Hardman for her support over the last three years.

Gregory Bogdanis for his collaboration in the analysis of muscle metabolites. His friendship throughout difficult times is very much appreciated.

Wendy Wilson for her assistance in the completion of the experimental work and analysis of plasma catecholamines.

Dr Paul Greenhaff, Dr Dimitru Constantin-Teodosiu and Anna Casey (Department of Physiology and Pharmacology, Medical School, Nottingham Unibersity) for their assistance in the determination of muscle glycogen in single fibres.

Dr Leslie Boobis (Sunderland Hospital) for performing the muscle biopsies required for these studies.

My sponsors SmithKline Beecham for their financial support throughout these years.

All the members of the Sports Science research Group at Loughborough University for their assistance and support in the completion of the experimental work.

All the individuals who volunteered to participate in these experiments.

Karen Rodkin for proof reading of the chapters of this thesis.

Without the help of those enlisted above this thesis would not have been completed. 


\section{LIST OF CONTENTS}

Page No.

\section{CHAPTER 1 INTRODUCTION}

CHAPTER 2 REVIEW OF LITERATURE

2.1 Introduction

4

2.2 Regulation of carbohydrate ( $\mathrm{CHO}$ ) metabolism

2.3 Blood glucose utilization during prolonged exercise

2.4 Fatigue during prolonged exercise

2.5 Influence of $\mathrm{CHO}$ ingestion during exercise on endurance capacity and performance

2.6 Factors affecting gastric emptying rate and intestinal absorption of $\mathrm{CHO}$ solutions

2.7 Influence of dehydration and fluid ingestion on temperature regulation

2.8 Summary

3.1 Introduction

3.2 Experimental testing procedures

3.3 Collection, treatment, storage and analysis of blood samples

3.4 Collection, treatment, storage and analysis of muscle samples

CHAPTER 4 INFLUENCE OF CHO-ELECTROLYTE DRINKS ON MARATHON RUNNING PERFORMANCE

4.1 Introduction 50

4.2 Methods 52

4.3 Results 55

4.4 Discussion 66 
CHAPTER 5 INFLUENCE OF CHO INGESTION ON RUNNING ENDURANCE CAPACITY

5.1 Introduction

70

$\begin{array}{lll}5.2 & \text { Methods } & 71\end{array}$

$\begin{array}{lll}5.3 & \text { Results } & 74\end{array}$

5.4 Discussion $\quad 83$

CHAPTER 6 EFFECT OF CHO INGESTION ON MUSCLE GLYCOGEN UTILIZATION IN TYPE I AND TYPE II FIBRES

$\begin{array}{lll}6.1 & \text { Introduction } & 88\end{array}$

6.2 Methods 89

6.3 Results 93

$\begin{array}{lll}6.4 & \text { Discussion } & 100\end{array}$

CHAPTER 7 CHO INGESTION AND MUSCLE GLYCOGEN DEPLETION PATTERN IN DIFFERENT FIBRE TYPES

$\begin{array}{lll}7.1 & \text { Introduction } & 106\end{array}$

$\begin{array}{lll}7.2 & \text { Methods } & 108\end{array}$

$\begin{array}{lll}7.3 & \text { Results } & 112\end{array}$

7.4 Discussion . 124

$\begin{array}{lll}\text { CHAPTER } 8 & \text { GENERAL SUMMARY }\end{array}$

$\begin{array}{ll}\text { REFERENCES } & 138\end{array}$

$\begin{array}{ll}\text { APPENDICES } & 163\end{array}$

A Mixed muscle metabolite assays $\quad 163$

B Spectrophotometric assays for validation of standards . 175

C Single muscle fibre glycogen assay $\quad 181$ 


\section{LIST OF FIGURES}

Figure

Page No.

4.1 Schematic illustration of the protocol of study 1

54

4.2 Running speeds for water (W), $6.9 \% \mathrm{CHO}$ (B) and $5.5 \% \mathrm{CHO}(\mathrm{A})$ trials during the $42.2-\mathrm{km}$ run

61

4.3 Blood glucose concentrations for W, B and A trials during the $42.2-\mathrm{km}$ run

4.4 Blood lactate concentrations for W, B and A trials during the $42.2-\mathrm{km}$ run

4.5 Plasma ammonia concentrations for W, B and A trials during the $42.2-\mathrm{km}$ run

4.6 Plasma Free Fatty Acids (FFA) concentrations

for $\mathrm{W}, \mathrm{B}$ and $\mathrm{A}$ trials during the $42.2 \mathrm{-km}$ run

4.7 Plasma glycerol concentrations for W, B and A trials during the $42.2-\mathrm{km}$ run

4.8 Plasma creatine kinase concentrations for

$\mathrm{W}, \mathrm{B}$ and $\mathrm{A}$ trials during the $42.2-\mathrm{km}$ run

5.1 Schematic illustration of the protocol of study 2

5.2 Times to exhaustion for the water (W), $6.9 \%$

$\mathrm{CHO}(\mathrm{C})$ and $5.5 \% \mathrm{CHO}(\mathrm{A})$ trials

5.3 Blood glucose concentrations for the W, A and

$C$ trials during the run to exhaustion

5.4 Blood lactate concentrations for the W, A and

$\mathrm{C}$ trials during the run to exhaustion 
5.5 Plasma FFA concentrations for the W, A and C trials during the run to exhaustion

5.6 Plasma glycerol concentrations for the W, A and $C$ trials during the run to exhaustion

6.1 Schematic illustration of the protocol of study 3

6.2 Blood glucose concentrations during the water (W) and $\mathrm{CHO}$ trials

6.3 Plasma insulin concentrations during the $\mathrm{W}$ and CHO trials

6.4 Blood lactate concentrations during the $\mathrm{W}$ and $\mathrm{CHO}$ trials

6.5 Plasma FFA concentrations during the $\mathrm{W}$ and $\mathrm{CHO}$ trials

6.6 Plasma glycerol concentrations during the $\mathrm{W}$ and $\mathrm{CHO}$ trials

7.1 Schematic illustration of the protocol of study 4

7.2 Blood glucose concentrations during the placebo (PL) and $\mathrm{CHO}$ trials

7.3 Serum insulin concentrations during the PL and $\mathrm{CHO}$ trials

7.4 Plasma FFA concentrations during the PL and $\mathrm{CHO}$ trials

7.5 Plasma glycerol concentrations during the PL and $\mathrm{CHO}$ trials 
7.6 Muscle glycogen concentrations at rest and exhaustion in the PL trial and at rest, the point coinciding with PL exhaustion and exhaustion in the $\mathrm{CHO}$ trial

7.7 Glycogen utilization rates in Type I and Type II fibres during prolonged running exercise without $\mathrm{CHO}$ ingestion

7.8 Glycogen utilization rates in Type I and Type II fibres during prolonged running exercise with $\mathrm{CHO}$ ingestion

8.1 Physiological and metabolic responses to prolonged running without $\mathrm{CHO}$ ingestion

8.2 Physiological and metabolic responses to prolonged running with $\mathrm{CHO}$ ingestion 


\section{LIST OF TABLES}

Table

Page No.

2.1 The effect of $\mathrm{CHO}$ ingestion during prolonged

cycling on endurance capacity

2.2 The effect of $\mathrm{CHO}$ ingestion during continuous or intermittent prolonged cycling on subsequent ability to perform a brief bout of of high intensity exercise

2.3 The effect of $\mathrm{CHO}$ ingestion during prolonged running on endurance capacity

2.4 The effect of $\mathrm{CHO}$ ingestion during prolonged running on endurance performance

3.1 CHO composition of the solutions used in the studies of this thesis

3.2 Coefficient of variation of blood, plasma and serum metabolite assays

3.3 Coefficient of variation of muscle metabolite assays

4.1 Performance times for the water (W), 6.9\% $\mathrm{CHO}(\mathrm{B})$ and $5.5 \% \mathrm{CHO}(\mathrm{A})$ trials during the $42.2-\mathrm{km}$ run

4.2 Oxygen uptake ( $\left.\mathrm{VO}_{2}\right), \% \mathrm{VO}_{2}$ max, respiratory exchange ratio (RER) and heart rate (HR) for the $W, B$ and $A$ trials during the $42.2-\mathrm{km}$ run

4.3 Plasma insulin, cortisol, growth hormone, adrenaline and nor-adrenaline concentrations in the $W, B$ and A trials during the $42.2-\mathrm{km}$ run 
5.1 $\mathrm{VO}_{2}, \% \mathrm{VO}_{2}$ max, RER, $\mathrm{CHO}$ oxidation rates and $\mathrm{HR}$ for the water $(\mathrm{W}), 6.9 \% \mathrm{CHO}(\mathrm{C})$ and $5.5 \% \mathrm{CHO}(\mathrm{A})$ trials during the run to exhaustion

5.2 Plasma ammonia, serum insulin, cortisol and growth hormone, and plasma catecholamine concentrations for the $\mathrm{W}, \mathrm{A}$ and $\mathrm{C}$ trials during the run to exhaustion

5.3 Plasma sodium and potassium concentrations for the $\mathrm{W}, \mathrm{A}$ and $\mathrm{C}$ trials during the run to exhaustion

6.1 \% VO 2 max, RER, $\mathrm{CHO}$ oxidation rates and heart rate during the water $(\mathrm{W})$ and $\mathrm{CHO}$ trials

6.2 Muscle metabolite concentrations before and after a 60 -min run in the $\mathrm{W}$ and $\mathrm{CHO}$ trials

6.3 Muscle glycogen concentrations in Type I and Type II fibres before and after a 60-min run in the $\mathrm{W}$ and $\mathrm{CHO}$ trials

$7.1 \mathrm{VO}_{2}, \% \mathrm{VO}_{2}$ max, $\mathrm{RER}, \mathrm{CHO}$ and fat oxidation rates, and $\mathrm{HR}$ for the placebo (PL) and 5.5\% $\mathrm{CHO}$ trials during the run to exhaustion

7.2 Blood lactate, serum sodium and potassium concentrations for the PL and CHO trials during the run to exhaustion

7.3 Muscle glycogen concentrations in Type I and Type II fibres at rest and exhaustion in the PL trial, and at rest, the time coinciding with PL exhaustion and exhaustion in the $\mathrm{CHO}$ trial

7.4 Muscle metabolite concentrations at rest and exhaustion in the PL trial, and at rest, the time coinciding with PL exhaustion and exhaustion in the $\mathrm{CHO}$ trial 


\section{CHAPTER 1}

\section{INTRODUCTION}

Prolonged running is an activity associated with a wide range of sports. Since any movement by the human body is brought about by converting chemical energy into mechanical energy, it follows that the ability to sustain a given level of effort for prolonged period of time, i.e. marathon running, is met by a continuous supply of chemical energy to the working muscles. This continuous supply of energy, in turn, would depend on the body's availability of certain energy substrates.

During submaximal exercise, carbohydrate and fat are the main energy substrates. Since the maximum rate of energy production from fat is almost half of that of carbohydrate (Jones and Round, 1992), carbohydrate is the main fuel during prolonged intense exercise. Although, body carbohydrate stores are limited, factors such as diet could increase these stores and training preserve their utilization. There is a limit, however, to ability to sustain a given level of effort for a prolonged period of time. Eventually, fatigue i.e. the failure to maintain the required energy output, will occur. Fatigue is a complex phenomenon which could be either central or peripheral or both in origin. Most of the experimental research has been focused on the peripheral aspect of fatigue. Considering the limited carbohydrate stores in the human body, it is not surprising that fatigue during prolonged heavy exercise has been associated with glycogen depletion in working muscles (Hermansen et al. 1967). Over the last decade, carbohydrate ingestion during exercise has become a very popular dietary practice aiming at the supplementation of the body's limited carbohydrate stores (Coyle et al. 1986; Maughan et al. 1989; Sasaki et al. 1987a; Williams et al. 1990).

Prolonged heavy exercise not only presents a challenge to the adequacy of the body's limited glycogen stores but also severely tests capacity for temperature regulation. Sweating plays a central role in thermoregulation but, during prolonged exercise, it is inevitably accompanied by dehydration. Dehydration decreases the ability of the individual to cope with exerciseinduced heat production because skin blood flow decreases and hence the 
capacity for heat loss is reduced (Coyle and Montain, 1992a). As a result core temperature increases and the person experiences intolerable discomfort which leads to a decrease in exercise capacity which may occur before muscle glycogen is reduced to critically low concentrations.

Drinking carbohydrate-electrolyte beverages during exercise has the advantage that they not only provide a way of supplementing the body's limited carbohydrate stores but they also help off-set fluid lost from sweating and so delay the onset of severe dehydration (Coyle and Montain, 1992b). The performance benefits of drinking carbohydrate-electrolyte solutions during prolonged submaximal cycling have been clearly confirmed in several studies (Coyle et al. 1983; 1986; Maughan et al. 1989). A wide range of concentrations of carbohydrate solutions have been shown to be effective in delaying fatigue and increasing endurance capacity (Lamb and Brodowicz, 1986).

However, relatively few studies examined the influences of drinking carbohydrate-electrolyte solutions on running endurance. Conflicting results have been obtained from these studies. The capacity for fluid ingestion is less during running than cycling because of the abdominal discomfort which occurs when the stomach is full. This is probably the main reason why runners tend to drink only small amounts of fluid during prolonged exercise (Bebb et al. 1984). Furthermore, the mechanism underlying the ergogenic effect of ingesting carbohydrate during exercise is not very clear.

The aim of these studies was to investigate the performance, physiological and metabolic responses to carbohydrate ingestion during prolonged treadmill running, in order to examine the relationship between carbohydrate ingestion and fatigue during this type of exercise.

This thesis is presented in seven main chapters. The review of literature (Chapter 2) deals with the effect of carbohydrate ingestion on endurance capacity and performance, metabolism and temperature regulation during prolonged exercise. The factors associated with fatigue and the availability of the ingested carbohydrate are also examined. 
The General methods chapter (Chapter 3 ) describes the equipment and the testing procedures used during the administration of the experimental tests, and the procedures associated with collection and analysis of blood and muscle samples.

The main aims of the first and second studies (Chapters 4 and 5) were (i) to investigate the influence of carbohydrate ingestion on a marathon running performance and a treadmill run at $70 \% \mathrm{VO}_{2} \max$ to exhaustion, respectively, and (ii) to describe some of the physiological and metabolic changes occuring during prolonged running with or without carbohydrate ingestion.

The purpose of the third study (Chapter 6) was to examine the effect of carbohydrate ingestion on glycogen utilization in different fibre types during a non-fatiguing exercise bout in order to establish whether or not sparing of muscle glycogen is one of the mechanisms by which $\mathrm{CHO}$ ingestion exerts its ergogenic effect.

The main aim of the last study (Chapter 7) was to examine whether or not glycogen sparing occurs during prolonged running to exhaustion and not simply early in exercise. An additional purpose was to compare the metabolic environment within the working muscles at the point of fatigue during running exercise without carbohydrate ingestion (PL) and at the point which coincided with PL exhaustion, and at exhaustion during running exercise with carbohydrate ingestion.

The last chapter of this thesis (Chapter 8) summarises the findings of all studies and addresses some of the questions on which future research should focus. 


\section{CHAPTER 2}

\section{REVIEW OF LITERATURE}

\subsection{INTRODUCTION}

The main aim of this chapter was to draw together some of the findings from several studies conducted over the last few decades to present a framework of our knowledge regarding the relationship between carbohydrate ingestion, ability to perform prolonged exercise and fatigue during this type of exercise.

This review of literature has been divided into six main sections. The first section (section 2.2) examines the regulatory mechanisms of carbohydrate (CHO) metabolism. Section 2.3 deals with blood glucose utilization during prolonged exercise, whereas section 2.4 examines the possible causes of fatigue during this type of exercise. The influence of $\mathrm{CHO}$ ingestion during exercise on endurance capacity and performance is reviewed in section 2.5. The next section of this review (section 2.6) examines the factors affecting gastric emptying rate and intestinal absorption of orally ingested $\mathrm{CHO}$ solutions. Finally, section 2.7 deals with the influence of dehydration and fluid ingestion on temperature regulation during prolonged exercise.

The terms endurance capacity and endurance performance have been used interchangeably in the literature. In this thesis, however, the two terms have been distinguished. Endurance capacity is referred to the time taken to reach exhaustion while exercising at a constant speed or work load, whereas endurance performance is the time taken to complete a pre-determined task (i.e. a $30-\mathrm{km}$ run). In this chapter, concentrations of muscle metabolites are expressed as mmol.kg dry muscle $(\mathrm{dm})^{-1}$. 


\subsection{REGULATION OF CARBOHYDRATE METABOLISM}

The successful completion of any human physical movement demands the transformation of chemical energy to mechanical energy in skeletal muscles at rates appropriate to their needs. The source of this chemical energy is the hydrolysis of adrenosine-5'-triphosphate (ATP). The amount of ATP stored in the muscle is limited and would only last for a few seconds of exercise (Newsholme and Start, 1973). Therefore, if the work rate is to be maintained for prolonged period of time then ATP must be regenerated continuously at the same rate as it is broken down. This continuous supply of energy would place a great demand on the human body's energy substrates for muscle contraction. During prolonged submaximal exercise glucose, glycogen and fatty acids stored as triglycerides are the main energy substrates. Of these, glycogen is the most important fuel for exercise requiring an oxygen uptake between 60 $100 \%$ of maximum (Saltin and Karlsson, 1971).

In this review, glycogenolysis is referred to as the metabolic pathway which involves the breakdown of glycogen to glucose-6-phosphate (G6P), whereas glycolysis involves the breakdown of G6P to pyruvate formation. The degradation of glucose to pyruvate is called glucolysis.

Regulation of glycogenolysis: Muscle glycogen breakdown during exercise is regulated by internally (within the muscle) and externally situated mechanisms. Glycogen degradation is catalyzed by the glycogen phosphorylase enzyme which exists in two forms, $\underline{a}$ (active) and $\underline{b}$ (inactive). Activation of phosphorylase is regulated by another enzyme, namely phosphorylase-bkinase. During muscle contraction, phosphorylase-b-kinase is activated by calcium released from the sarcoplasmic reticulum (Danforth, 1965). In addition to calcium, increased intramuscular concentrations of adenosine monophosphate (AMP), inosine monophosphate (IMP) and inorganic phosphate $(\mathrm{Pi})$ during exercise exert allosteric effects on phosphorylase activity (Aragon et al. 1980; Chasiotis et al. 1982). It has been observed that IMP and Pi increase even during prolonged exercise at $60-70 \%$ of $\mathrm{VO}_{2} \max$ (Broberg and Sahlin, 1989). Recently, however, it has been suggested that factors other than the extent of transformation of phosphorylase $\underline{b}$ to $\mathbf{a}$ and the effect of Pi on the activity of the enzyme might be involved in the regulation of the rate of glycogen breakdown during exercise (Ren and Hultman, 1989). 
The rate of muscle glycogen breakdown is also under hormonal regulation. Adrenaline seems to play an important role in muscle glycogenolysis during exercise. Its effect on the activation of phosphorylase is mediated through betaadrenergic receptor stimulation (Dietz et al. 1980). Infusion of adrenaline has been shown to enhance muscle glycogen breakdown during exercise in man (Jansson et al. 1986). It seems, however, that during intense muscular contraction the adrenaline effect on glycogen breakdown is more important in Type I (slow twitch) rather than Type II (fast twitch) fibres (Greenhaff et al. 1991). On the other hand, at rest or during non-intense muscular contraction adrenaline seems to be more important in Type II rather than Type I fibres (Chasiotis, 1985; Richter et al. 1982). It has been suggested that contractioninduced glycogen breakdown is more important during the early stages of exercise, whereas adrenaline-induced glycogen breakdown is more important during prolonged contractile activity (Hargreaves and Richter, 1988). It has also been shown that although the a form of phosphorylase quickly reverts to the $b$ form, glycogen degradation continues to occur (Conlee et al. 1979). This could be very important for prolonged submaximal exercise. During this type of exercise a progressive increase in plasma adrenaline concentration has been observed (Galbo et al. 1975; Kjaer et al. 1985). Furthermore, Type I fibres are the main fibres recruited during this type of exercise. As the exercise continues and depletion of glycogen in Type I fibres occurs, the elevated circulating adrenaline may stimulate an increased glycogen breakdown in fast-twitch muscle fibres to maintain a high rate of glycogenolysis (Vollestad et al. 1984).

Since insulin promotes glycogen synthesis, it is not known to what extent the decrease in plasma insulin concentration observed during prolonged heavy exercise could affect the rate of glycogen utilization (Hargreaves and Richter, 1988). The rate of muscle glycogen utilization during prolonged exercise has also been shown to be directly related to the pre-exercise muscle glycogen levels in some (Gollnick et al. 1972; Richter and Galbo, 1986) but not all cases (Saltin et al. 1992), and to reduced plasma FFA availability (Bergstrom et al. 1969). On the other hand, an increase in the plasma FFA levels (Costill et al. 1977; Hickson et al. 1977) or intravenous infusion of glucose (Bergstrom and Hultman, 1967) resulted in a decreased rate of muscle glycogen utilization.

Regulation of glucolysis: Glucose uptake and its phosphorylation to G6P are the main regulatory steps in exogenous glucose utilization. Glucose uptake: Muscle contractility and circulating levels of insulin stimulate glucose 
transport across the sarcolemma. The effects of these factors are additive (Nesher et al. 1985) and are possibly brought about independently. It has been shown that insulin is not required for contraction-induced glucose uptake (Ploug et al. 1984). Both contractile activity and insulin promote the translocation of GLUT-4 glucose transporters from an intracellular storage site to the plasma membrane (Douen et al. 1990). It has also been suggested that contraction and insulin translocate these transporters from two different pools (Douen et al. 1989; 1990). Both factors could also alter the affinity and activity of these transporters (Bonen et al. 1990). In rats it has been shown that Type I fibres possess a higher content of GLUT-4 compared with Type II fibres (Henriksen et al. 1990). As a result, during contraction slow twitch muscles exhibit higher glucose uptake compared with fast twitch muscles (Bonen et al. 1990).

Glucose phosphorylation: The hexokinase enzyme catalyzes the phosphorylation of glucose as it enters the muscle cell. It has been shown that glucose transporters and hexokinase are closely associated at the plasma membrane of many cells; this coupling appears so efficient that $80 \%$ of glucose entering the cell is phosphorylated to G6P before entering the cytosol (Carruthers, 1990). Recent evidence suggests that G6P concentration is the major inhibitor of hexokinase in human skeletal muscle (Katz et al. 1991). A negative correlation exists between blood glucose uptake by the working muscles and muscle G6P concentrations; this relationship is linear for concentrations of G6P between 0.9 and 3.5 mmol.kg dm-1 (Gollnick et al. 1981). It has been shown that during the early phase of prolonged exercise the limiting step for blood glucose utilization is glucose phosphorylation to G6P since the high concentration of G6P observed during that period (as a result of rapid glycogenolysis) would inhibit hexokinase. During the latter phase of exercise, however, the limiting step for glucose utilization shifts from phosporylation to glucose transport (Katz et al. 1991).

Regulation of glycolysis: The activity of phosphofructokinase (PFK) dictates the overall rate of glycolytic flux to pyruvate. The enzyme activity is low in resting muscle and is inhibited by ATP, $\mathrm{PCr}, \mathrm{Mg}^{2+}, \mathrm{H}^{+}$, citrate and some of the glycolytic 3-carbon intermediates, but activated by AMP, ADP, $\mathrm{Pi}$, cyclic AMP, $\mathrm{NH}_{4}{ }^{+}, \mathrm{K}^{+}, \mathrm{F}-6-\mathrm{P}$ and F-1,6-diphosphate (Hultman et al. 1987). Phosphofructokinase is very important in coupling glycolysis to a number of metabolic pathways, namely: $i$ / the rate of lactate accumulation; ii/ function of 
the tricarboxylic acid acid cycle (TCA); iii/ ATP production by mitochondrial oxidative phosphorylation; iv/ amino acid metabolism; $v$ / buffering of the mitochondrial redox state to prevent loss of reducing equivalents from the mitochondrion; and vi/ integration of the local muscle metabolism to the whole body metabolism by controlling hormonal signals or signals indirectly related to substrate availability (Stanley and Connett, 1991).

Regulation of hepatic glucose production: The liver is assumed to be the only significant source of blood glucose both at rest and during exercise. In general, circulating hormones (insulin, glucagon and catecholamines) and autonomic nerve impulses in liver tissue have been suggested as potential regulators of hepatic glucose production during exercise (Hultman and Harris, 1988). Recently, the role of the neural input to the liver and adrenaline stimulation in regulating hepatic glucose production has been questioned (Wasserman et al. 1990; Kjaer et al. 1990). Glucagon concentration in the portal vein (Wasserman et al. 1989) and, in particular, the glucagon-to-insulin molar ratio have been suggested as important regulators of hepatic glucose production during exercise (Wasserman et al. 1984; Miles et al. 1992). It seems, however, that catecholamine-to-insulin molar ratio might also be important during the early phase of prolonged exercise (Miles et al. 1992). 


\subsection{BLOOD GLUCOSE UTILIZATION DURING PROLONGED EXERCISE}

Blood glucose utilization in the fasted state is mainly a function of the intensity and duration of exercise (Wahren, 1971; Katz et al. 1986; Ahlborg and Felig, 1982). Blood glucose utilization has a positive curvilinear relationship with exercise intensity (Coggan, 1991). During the early stages (up to $40 \mathrm{~min}$ ) of prolonged exercise at about $70 \% \mathrm{VO}_{2}$ max glucose utilization by the working muscles averages 0.5 g.min ${ }^{-1}$ (Broberg and Sahlin, 1989). As blood glucose utilization also increases with the duration of exercise (Ahlborg and Felig, 1982), it has been shown that towards the latter stages of exercise at about $70 \%$ $\mathrm{VO}_{2}$ max it might reach values of $1.2 \mathrm{~g}_{\mathrm{min}} \mathrm{m}^{-1}$ (Coggan and Coyle, 1987; Coggan et al. 1991). At a time when glycogen is very low, this could account for up to $75 \%$ of total $\mathrm{CHO}$ oxidation rate. These findings emphasize the importance of blood glucose as an energy substrate during prolonged exercise.

Apart from intensity and duration of exercise other factors could also affect the rate of blood glucose oxidation during exercise. Briefly, glucose uptake is inversely related to the muscle mass involved (Richter et al. 1988) and to the muscle glycogen concentration (Richter and Galbo, 1986). There is some evidence that a low $\mathrm{CHO}$ diet would lower the blood glucose utilization, whereas a high $\mathrm{CHO}$ diet might increase it (Galbo et al. 1979). Endurance training has also been shown to decrease blood glucose utilization (Coggan et al. 1990).

The effect of $\mathrm{CHO}$ ingestion during exercise on blood glucose utilization has been studied extensively using ${ }^{14} \mathrm{C}$ or ${ }^{13} \mathrm{C}$-labeled exogenous $\mathrm{CHO}$. The amount of this $\mathrm{CHO}$ oxidised can be computed from the ratio of the tracer released in the form of ${ }^{13} \mathrm{CO}_{2}$ or ${ }^{14} \mathrm{CO}_{2}$ to the tracer administered; the amount of tracer trapped within the body and the amount of tracer released in the form of $\mathrm{CO}_{2}$ but which comes from the oxidation of endogenous substrates (background emmision of tracer) should also be taken into account (Peronnet et al. 1992).

Early studies using ${ }^{14} \mathrm{C}$-labeled exogenous $\mathrm{CHO}$ showed that a very small fraction of the ingested $\mathrm{CHO}$ was actually oxidised during exercise between 50 and $70 \% \mathrm{VO}_{2}$ max; values in the range of 0.05 and 0.11 g.min-1 were reported accounting for only $5 \%$ of total CHO oxidation (Costill et al. 1973; Van Handel et al. 1980). It has been suggested that this is probably an underestimation of 
the exogenous $\mathrm{CHO}$ oxidation resulted from either a technical problem associated with the labeling of the exogenous $\mathrm{CHO}$ (Peronnet et al. 1992) or the slow rate of passage of ${ }^{14} \mathrm{CO}_{2}$ through the body's bicarbonate pool (Coggan and Coyle, 1991). Recent studies, however, using ${ }^{14} \mathrm{C}$-labeled exogenous $\mathrm{CHO}$ showed that during cycling at $70 \% \mathrm{VO}_{2}$ max exogenous $\mathrm{CHO}$ was oxidised at a much higher rate (peak $1.0 \mathrm{~g} \cdot \mathrm{min}^{-1}$ ) and accounted for almost $20 \%$ of the total CHO oxidation (Hawley et al. 1992). In another study a peak rate of exogenous CHO oxidation of 1.8 g.min ${ }^{-1}$ was reported following the ingestion of an insoluble starch suspension enriched with a ${ }^{14} \mathrm{C}$-labeled soluble starch (Hawley et al. 1991). These latter values were extremely high and might have been over-estimated. Indeed, it has been suggested recently that the addition of soluble tracer into a solution containing insoluble type of $\mathrm{CHO}$ might lead to over-estimation of exogenous oxidation rates (Jeukendrup et al. 1993).

Far more studies, however, have been conducted using ${ }^{13} \mathrm{C}$-labeled exogenous CHO (Pirnay et al. 1977a; 1977b; 1982; Krzentowski et al. 1984; Pallicarakis et al. 1986; Massicotte et al. 1986; 1989). In these studies the rates of oxidation of the exogenous $\mathrm{CHO}$ ranged between 0.18 and $0.80 \mathrm{~g} \cdot \mathrm{min}^{-1}$ accounting for $13-87.5 \%$ of the total $\mathrm{CHO}$ metabolism. The exercise intensity (at least in the range of 22 to $65 \% \mathrm{VO}_{2}$ max) and the total amount of $\mathrm{CHO}$ ingested appear to be the most important factors affecting the rate of oxidation (Peronnet et al. 1992; Pallicarakis et al. 1986). However, these results should be interpreted with caution because these studies did not take into account changes in the background emmision of tracer due to modification of the composition of the mixture of endogenous substrates; as a result they have greatly over-estimated the actual exogenous CHO oxidation (Peronnet et al. 1990; 1992). In order to solve this problem a new computation procedure, which takes into account changes in the background emmision of expired $\mathrm{CO}_{2}$ by using either two levels of low enrichment or one very high level of enrichment of the glucose ingested, was developed (Peronnet et al. 1990). Using this procedure and conditions similar to those employed by Costill et al. (1973), exogenous $\mathrm{CHO}$ utilization during cycling at $67-68 \% \mathrm{VO}_{2}$ max averaged $0.23 \mathrm{~g} \cdot \mathrm{min}^{-1}$ which represented $43-50 \%$ of total glucose load and contributed 6-9\% of total energy metabolism (Peronnet et al. 1992; 1993).

Other studies have used the glycaemic clamp technique to quantify the rate of exogenous glucose utilization; the assumption being that the exogenous glucose is oxidised at the infusion rate required to maintain blood glucose at a 
certain level (Coggan and Coyle, 1987; Coyle et al. 1991). The results from these studies revealed that towards the end of prolonged exercise maintaining blood glucose at euglycaemic levels $\left(5 \mathrm{mmol} . \mathrm{I}^{-1}\right)$ would require the infusion of glucose at a rate of $1.1 \mathrm{~g} \cdot \mathrm{min}^{-1}$, whereas maintaining it at $10 \mathrm{mmol.1}-1$ would require the infusion at a rate of $2.6 \mathrm{~g} \cdot \mathrm{min}^{-1}$. These results also showed the great capacity of the working muscles to oxidise blood glucose at a time when muscle glycogen becomes depleted and thus further emphasised the importance of $\mathrm{CHO}$ ingestion for endurance capacity during prolonged exercise. 


\subsection{FATIGUE DURING PROLONGED EXERCISE}

The re-introduction of the Duchene needle biopsy technique (Bergstrom, 1962) initiated a series of investigations aiming at the direct assessment of the changes in muscle glycogen content during prolonged exercise. The results of those studies suggested that during cycling to exhaustion at relatively high exercise intensities (70-80\% $\mathrm{VO}_{2} \mathrm{max}$ ) the depletion of glycogen stores in the human quadriceps muscle is limiting the performance of prolonged strenuous work (Ahlborg et al. 1967; Hermansen et al. 1967). Furthermore, Saltin and Karlsson (1971) observed that during cycling exercise lasting 45-200 minutes (89-65\% of $\mathrm{VO}_{2}$ max) exhaustion coincided with depletion of glycogen in active skeletal muscles.

In some studies a significant amount of muscle glycogen remained at the end of prolonged exhaustive exercise (Costill et al. 1973a; Sherman et al. 1983; Coyle et al. 1986). Histochemical determination of glycogen in different fibre types revealed that Type I (slow twitch) but not Type II (fast twitch) fibres were glycogen depleted. On the basis of these results, a selective depletion of the glycogen stores of those muscle fibres recruited during exercise has been suggested as a possible cause of fatigue (Conlee, 1987). Consequently, determination of total muscle glycogen does not always reflect the glycogen utilization or the metabolic profile of the fibres recruited to sustain a given exercise task (Costill et al. 1973a).

On the basis of histochemically determined changes in the glycogen concentration of individual fibres from the working muscles, Gollnick et al. (1973) demonstrated a preferential pattern of fibre use in human quadriceps muscle during cycling to exhaustion at $65 \%$ of $\mathrm{VO}_{2}$ max. The Type I fibres were the first to become depleted of their glycogen and subsequently a sequential glycogen loss from Type Il fibres to a marked extent was observed.

In a recent study (Vollestad et al. 1984), the glycogen depletion patterns in Type I and in the subgroups of Type II fibres (IIA, IIAB and IIC) were histochemically assessed during intermittent cycling exercise to exhaustion at $75 \%$ of $\mathrm{VO}_{2}$ max. It was suggested that the Type I and Type IIA fibres were recruited from the start of exercise as indicated by the same glycogen depletion rate found between the two fibre groups. At the later stages of the exercise Type IIAB and IIB fibres were recruited suggesting that the threshold for activation of those fibres 
decreased as the exercise proceded and Type I and IIA fibres became depleted of their glycogen stores (Vollestad et al. 1984).

However, it appears that differences exist between prolonged running and cycling exercise (Costill et al. 1973a). After $30 \mathrm{Km} \mathrm{(2.5} \mathrm{h)} \mathrm{of} \mathrm{road} \mathrm{running} \mathrm{at}$ $83 \%$ of $\mathrm{VO}_{2}$ max, considerable glycogen remained in the quadriceps muscle when the subjects were near exhaustion (Costill et al. 1973a). In that study, however, a marked reduction in the glycogen stores was evident only in Type I fibres. In contrast to prolonged cycling (Gollnick et al. 1973) a minor reduction in muscle glycogen occurred in Type II fibres. Even after a marathon road race the glycogen content in the gastrocnemius muscle in 10 runners was well above zero (108 $\mathrm{mmol} \cdot \mathrm{kg} \mathrm{dm}^{-1}$; Sherman et al. 1983). Similarly, a significant amount of glycogen in the gastrocnemius muscle was observed at the end of exhaustive treadmill running; neither Type I nor Type II fibres were found depleted (Madsen et al. 1990).

The mechanism, however, which links glycogen depletion and failure to sustain the contractile tension needed for aerobic work performance is not fully understood. Recently, it has been demonstrated (Broberg and Sahlin, 1989) that cycling to exhaustion at $70 \%$ of $\mathrm{VO}_{2}$ max results in a thirty two-fold increase in muscle inosine monophosphate (IMP) and seven-fold increase in ammonia $\left(\mathrm{NH}_{3}\right)$. Norman and his colleagues (1988) observed that this increase in the IMP content was restricted to the glycogen depleted fibres. Indeed, an inverse relationship seems to exist between IMP formation and glycogen depletion (Spencer et al. 1991). These findings indicated that low glycogen levels could increase the rate of adenine nucleotide degradation during prolonged exercise by enhancing the rate of AMP deamination. It was hypothesized, therefore, that failure of the glycolysis to rephosphorylate adenosine diphosphate (ADP) at a sufficient rate results in impairment of the . contraction process (Broberg and Sahlin, 1989).

Recently, it was shown that during exhaustive cycling at $75 \% \mathrm{VO}_{2}$ max muscle acetyl-CoA concentration, the substrate for tricarboxylic acid cycle, was constant throughout exercise and at exhaustion it was not different to the value observed during the first ferv minutes (Constantin-Teodosiu, 1992; Sahlin et al. 1990). It has also been shown that the increased acetyl-CoA/CoASH and $\mathrm{NADH} / \mathrm{NAD}^{+}$ratios during exercise do not inhibit the activation of pyruvate dehydrogenase complex (PDC) which catalyzes the oxidation of pyruvate to 
acetyl-CoA (Constantin-Teodosiu, 1992). Thus, acetyl-CoA formation does not seem to limit TCA cycle function and, hence, aerobic energy production. It was further observed that glycogen depletion may impair aerobic function by reducing the level of tricarboxylic acid cycle intermediates (TCAI) (Sahlin et al. 1990). Recently, it has been shown that oxidation of branched-chain amino acids (BCAA) in the working muscles is inversely related to the muscle glycogen content (Wagenmakers et al. 1991). Thus, an increased BCAA oxidation would be expected at the end of prolonged exercise when the muscle glycogen is low. It was hypothesised that an excessive activation of BCAA oxidation could lead to a decreased level of TCA intermediates and thus reduced aerobic energy production by draining the TCA cycle at the level of BCAA aminotransferase reaction in which 2-oxoglutarate (one of the TCAI) is used as an amino group aceptor (Wagenmakers et al. 1990; 1991).

It has been suggested, however, that glycogen depletion might not be the cause of fatigue in highly trained athletes during exhaustive treadmill running (Madsen et al. 1990). Loss of intracellular potassium and its effect on excitationcontraction process through decreased depolarization of muscle membrane has also been proposed as a mechanism of fatigue during prolonged exercise (Sjogaard et al. 1985; Sjogaard, 1986). Force generation could also be reduced during prolonged low-frequency stimulation by an insufficient calcium release from the sarcoplasmic reticulum (SR) due to depletion of phosphatidylinositol 4,5-biphosphate ( $\left.\mathrm{PIP}_{2}\right)$. During excitation of the sarcolemma the development of action potential leads to depolarization of transverse tubule (TT) and as a result $\mathrm{PIP}_{2}$, located at $\mathrm{TT}$, is hydrolysed to inositol triphosphate (InsP3) which is believed to be the signal for calcium release from SR (Donaldson, 1990).

The central assumption behind all these postulated mechanisms of fatigue during prolonged exercise is that inadequate force development is the result of a disfunction in excitation-contraction process within the muscle itself (peripheral fatigue). However, fatigue could also be central in origin (inadequate activation of the muscle by the central nervous system (CNS)). Alternatively, a feedback mechanism from the muscle could also alter motoneuron excitability and hence reduce central motor drive (Asmussen, 1979). Christensen and Hansen (1939) have suggested that hypoglycemia (blood glucose $<2.5 \mathrm{mM}$ ) could be the cause of fatigue during prolonged exercise at $60-65 \% \mathrm{VO}_{2}$ max by affecting the CNS. Contrary to the above 
suggestion, Felig et al. (1982) have shown that cycling exercise to exhaustion at $60-65 \% \mathrm{VO}_{2} \max$ can be continued in the presence of hypoglycemia.

Recently, it was hypothesized that an increased synthesis of the neurotransmitter serotonin (5-HT) in the brain could result in central fatigue during prolonged submaximal exercise (Newsholme et al. 1987). Brain serotonin has been associated with pain and arousal (Young, 1986). During prolonged exercise an increase in the plasma tryptophan to branched chain amino acid ratio is observed. Since both compete for the same carrier to cross the blood-brain barrier an increase in that ratio would lead to an increased amount of tryptophan crossing the blood-brain barrier; as tryptophan is a precursor for serotonin (5-HT) synthesis, an increased synthesis of 5-HT might occur (Newsholme et al. 1987), which could deteriorate the ability to sustain prolonged exhaustive exercise (Blomstrand et al. 1991).

It has also been suggested that prolonged heavy exercise could affect cardiac function leading to cardiac fatigue. Indeed, a reduced fractional shortening of the left ventricular wall and a reduced left ventricular diastolic dimension were observed immediately after a triathlon despite an unchanged blood pressure and wall stress (Douglas et al. 1987). There is also some evidence that during exercise of extreme duration the heart starts to use endogenous energy stores as opposed to rest or short-term exhaustive exercise where supply of blood-borne metabolites is probably adequate to meet the demands of the myocardium; it is not known, however, to what extent this could affect cardiac function (Kaijser, 1992).

Prolonged exercise performed in the heat could also lead to fatigue well before glycogen in the working muscles becomes depleted (Fink et al. 1975). Although early studies suggested that glycogen breakdown is accelerated in a hot compared with cold environment (Fink et al. 1975; Edwards et al. 1972), recent evidence has shown that glycogen utilization and other aspects of energy metabolism (i.e. plasma FFA and glucose uptake) as well as blood flow to the working muscles were similar both in the cold and hot environment (Nielsen et al. 1990). It was suggested, therefore, that fatigue during prolonged exercise at $60-70 \% \mathrm{VO}_{2}$. max performed in the heat is the result of impaired motor centre function or mental drive for motor performance due to high core temperature (Nielsen et al. 1990). 
In conclusion, although fatigue during prolonged exercise performed in a neutral environment could be either central or peripheral in origin, there is strong evidence accumulated over the last 25 years to suggest that fatigue. during this type of exercise coincides with depletion of glycogen in the working skeletal muscles. Although the exact link between glycogen depletion and fatigue is not fully understood, some recent evidence suggests that glycogen depletion impairs the muscle contraction process by reducing the level of tricarboxylic acid cycle intermediates (TCAI) and hence the rate of ATP resynthesis. 


\subsection{INFLUENCE OF CARBOHYDRATE INGESTION DURING EXERCISE ON ENDURANCE CAPACITY AND PERFORMANCE}

Carbohydrate supplementation during prolonged exercise is a widespread dietary practice among both recreational runners and endurance athletes. As discussed in the previous section, depletion of the limited stores of glycogen in the exercising skeletal muscle and/or hypoglycemia have been shown to coincide with fatigue during this type of exercise. Therefore, the rationale under-pinning the ingestion of any $\mathrm{CHO}$ solution during exercise is that in preventing hypoglycemia and providing an alternative fuel source which is immediately usable by the working muscles, the onset of fatigue could be delayed. Although, different exercise models (i.e. laboratory versus field protocols, intermittent versus continuous exercise) have been used to investigate the effect of $\mathrm{CHO}$ ingestion during exercise on endurance capacity and performance, most of the studies have used cycling as the mode of exercise. Despite the popularity of sports associated with prolonged running (i.e. marathon, ultramarathon, triathlon), it is only during the last decade the responses to $\mathrm{CHO}$ ingestion during running have been studied.

Cycling studies: As early as in 1939 Christensen and Hansen had reported that $200 \mathrm{~g}$ of glucose ingested late in exercise resulted in a reversal of hypoglycemic symptoms and an ability to exercise for an additional hour during prolonged exercise at $60-65 \% \mathrm{VO}_{2}$ max. This improvement in endurance capacity was attributed to disappearance of Central Nervous System (CNS) hypoglycemia since respiratory exchange ratio (RER) did not increase after glucose ingestion. More recently, it has been shown that cycling exercise to exhaustion at $60-65 \%$ $\mathrm{VO}_{2}$ max can be continued in the presence of hypoglycemia (blood glucose < $2.5 \mathrm{mM}$ ) and that although glucose ingestion during exercise prevents hypoglycemia, it does not consistently delay exhaustion (Felig et al. 1982).

With the exception of the above study (i.e. Felig et al. 1982) CHO ingestion during cycling exercise has been shown to significantly improve endurance capacity compared with water or placebo ingestion (Bjorkman et al. 1984; Coyle et al. 1983; 1986; Coggan and Coyle, 1989; Maughan et al. 1989; Wright et al. 1991). In these studies glucose, glucose polymer and glucose polymer-fructose solutions between 4 and $50 \%$ have been used (rate of $\mathrm{CHO}$ intake in the range of 22-111 g.h ${ }^{-1}$ ); the improvement in endurance capacity ranged between $17 \%$ 
and 33\% (Table 2.1). A dose-response relationship between $\mathrm{CHO}$ intake and improvement in capacity does not appear to exist.

When the time to complete a bout of high-intensity exercise following a prolonged continuous or intermittent cycling exercise was employed as a measure of endurance performance (Table 2.2), similar results have been obtained (Davis et al. 1988a; Fielding et al. 1985; Hargreaves et al. 1984; Murray et al. 1991; Mitchell et al. 1989a). These studies suggested that there is no doseresponse relationship between $\mathrm{CHO}$ ingestion rates and performance. It seems that $\mathrm{CHO}$ feeding at a rate of $25 \mathrm{~g} \cdot \mathrm{h}^{-1}$ is required to elicit an improvement in performance under these conditions.

When the measure of endurance performance has been defined as the total power output achieved in a certain amount of time, $\mathrm{CHO}$ ingestion $(90 \mathrm{~g}$ of maltodextrin and 90 or $45 \mathrm{~g}$ of maltodextrin-fructose solutions) failed to increase the total power output during $2 \mathrm{~h}$ of cycling exercise (Ivy et al. 1979; Flynn et al. 1987).

Walking and running studies: Ivy et al. (1983) have shown an increased endurance capacity during exhaustive walking exercise at $45 \% \mathrm{VO}_{2}$ max when $120 \mathrm{~g}$ of glucose polymer, as opposed to placebo, was ingested during exercise (299 $\mathrm{min}$ vs $268 \mathrm{~min}$ respectively, $12 \%$ increase).

Studies using running as the mode of exercise have produced conflicting results regarding the effect of $\mathrm{CHO}$ ingestion compared with water on endurance capacity (Table 2.3). Sasaki et al. (1987a) have observed an improved time to exhaustion at $80 \% \mathrm{VO}_{2} \max$ as a result of ingesting $90 \mathrm{~g}$ of sucrose compared with placebo. When a 5\% (3\% glucose polymers and $2 \%$ fructose) solution was given during a run to exhaustion at $85 \%$ of $\mathrm{HR}$ max an improved time was reported compared with either no fluid or water ingestion (Macaraeg, 1983). Recently, a 29\% improvement in the time to exhaustion was observed when a $7 \% \mathrm{CHO}$ solution was ingested during treadmill running at $80 \% \mathrm{VO}_{2}$ $\max$ (Wilber and Moffatt, 1992).

In contrast to the above results, Fruth and Gisolfi (1983) have shown no difference in running time to exhaustion at $70 \% \mathrm{VO} 2$ max when placebo, $20 \mathrm{~g}$ of glucose or $20 \mathrm{~g}$ of fructose (in a $10 \%$ solution) were ingested every $20 \mathrm{~min}$ of the run. However, there was a tendency for a shorter running time in the 
fructose trial compared with the other two trials. Riley et al. (1988) also failed to demonstrate an improved time to exhaustion at $70 \% \mathrm{VO}_{2}$ max following a 21-h fast, when a $7 \%$ CHO solution (5\% glucose polymer and $2 \%$ fructose) was ingested every $20 \mathrm{~min}$ of the run as opposed to placebo intake.

An experimental model which has been developed recently enables the subject to adjust the speed of the treadmill during the run (Table 2.4). Using this model (Williams et al. 1988; 1990), it has been observed that $50 \mathrm{~g}$ of $\mathrm{CHO}$ ingested either during a $30 \mathrm{~km}$ performance test or during a $2 \mathrm{~h}$ test did not result in significantly faster time or greater total distance covered respectively, compared with water ingestion. There was a tendency however for a greater running speed during the last $30 \mathrm{~min}$ and $10 \mathrm{~km}$ of the tests, respectively, when $\mathrm{CHO}$ was ingested.

Field studies: In a study conducted under actual cycling conditions (55.2 miles) (Edwards et al. 1984) 8 out of 9 world-class cyclists performed better when they ingested either glucose polymer or glucose polymer/fructose solutions every 25 min during each ride in comparison with water ingestion. Recently, Millard-Stafford et al. (1992) have observed an improvement in performance over a $40 \mathrm{~km}$ outdoor run when a $7 \% \mathrm{CHO}$ solution, as opposed to water, was ingested during the run. In a more recent study (Tsintzas et al. 1993) the ingestion of a $5 \%$ CHO solution every $5 \mathrm{~km}$ during a $30-\mathrm{km}$ outdoor race resulted also in an improved time compared with water (128 $\pm 19.9 \mathrm{~min}$ vs 131 \pm 18.7. $\mathrm{min}$, respectively) (Table 2.4 ). 
Table 2.1 : The effect of $\mathrm{CHO}$ ingestion during prolonged cycling exercise at $60-75 \% \mathrm{VO}_{2}$ max on endurance capacity

\begin{tabular}{lcccc}
\hline Study & n & $\begin{array}{c}\text { Type of CHO } \\
\text { ingested }\end{array}$ & $\begin{array}{c}\text { Total amount } \\
\text { of CHO ingested }\end{array}$ & Improvement \\
\hline Felig et al. 1982 & 19 & G & $110-220 \mathrm{~g}$ & ns \\
Coyle et al. 1983 & 10 & GP & $121 \mathrm{~g}$ & $17 \%$ \\
Bjorkman et al. 1984 & 8 & G & $105 \mathrm{~g}$ & $18 \%$ \\
Coyle et al. 1986 & 7 & GP & $406 \mathrm{~g}$ & $33 \%$ \\
Coggan \& Coyle, 1989 & 6 & GP+S & $211 \mathrm{~g}$ & $21 \%$ \\
Maughan et al. 1989 & 6 & G & $36 \mathrm{~g}$ & $19 \%$ \\
Wright et al. 1991 & 9 & GP+F & $175 \mathrm{~g}$ & $32 \%$ \\
\hline
\end{tabular}

$G=$ glucose,$G P=$ glucose polymer,$S=$ sucrose, $F=$ fructose, $n s=$ not significant

Table 2.2: The effect of $\mathrm{CHO}$ ingestion during continuous $(1,2,3)$ or intermittent $(4,5,6)$ prolonged cycling exercise at $50-75 \% \mathrm{VO}_{2}$ max on subsequent ability to perform a brief bout of high intensity exercise

\begin{tabular}{lcccc}
\hline Study & $\mathrm{n}$ & $\begin{array}{c}\text { Type of CHO } \\
\text { ingested }\end{array}$ & $\begin{array}{c}\text { Amount of } \\
\text { CHO ingested }\end{array}$ & Improvement \\
\hline (1) Davis et al. 1988a & 19 & S+G & $149 \mathrm{~g}$ & $9 \%$ \\
(2) Mitchell et al. 1989a & 10 & GP+F & $148 \mathrm{~g}$ & $13 \%$ \\
(3) Kingwell et al. 1989 & 9 & GP & $160 \mathrm{~g}$ & $\mathrm{~ns}$ \\
& 10 & $\mathrm{~S}$ & $172 \mathrm{~g}$ & $45 \%$ \\
(4) Hargreaves et al. 1984 & 9 & $\mathrm{~S}$ & $86 \mathrm{~g}$ & $49 \%$ \\
(5) Fielding et al. 1985 & 10 & G $+\mathrm{GP}$ & $52 \mathrm{~g}, 156 \mathrm{~g}$ & $6 \%, 7 \%$ \\
(6) Murray et al. 1991 & & & & \\
\hline
\end{tabular}

$G=$ glucose,$G P=$ glucose polymer,$S=$ sucrose, $F=$ fructose, $n s=$ not significant 
Table 2.3 : The effect of $\mathrm{CHO}$ ingestion during prolonged running exercise at $70-80 \% \mathrm{VO}_{2}$ max on endurance capacity

\begin{tabular}{lcccc}
\hline Study & $\mathbf{n}$ & $\begin{array}{c}\text { Type of CHO } \\
\text { ingested }\end{array}$ & $\begin{array}{c}\text { Amount of } \\
\text { CHO ingested }\end{array}$ & Improvement \\
\hline Macaraeg, 1983 & 12 & GP+F & $60 \mathrm{~g}$ & $31 \%$ \\
Sasaki et al. 1987a & 5 & $\mathrm{~S}$ & $90 \mathrm{~g}$ & $48 \%$ \\
Wilber and Moffatt, 1992 & 10 & $\mathrm{GP}+\mathrm{S}$ & $91 \mathrm{~g}$ & $29 \%$ \\
Fruth and Gisolfi, 1983 & 7 & $\mathrm{G}, \mathrm{F}$ & $140 \mathrm{~g}$ & $\mathrm{~ns}$ \\
Riley et al. 1988 & 9 & GP+F & $98 \mathrm{~g}$ & $\mathrm{~ns}$ \\
\hline
\end{tabular}

$\mathrm{G}=$ glucose, $\mathrm{GP}=$ glucose polymer $\mathrm{S= \text {sucrose }} \mathrm{F}=$ fructose, ns=not significant

Table 2.4 : The effect of $\mathrm{CHO}$ ingestion during prolonged running exercise on endurance performance (1,2=laboratory studies and 3,4=field studies)

\begin{tabular}{lccccc}
\hline Study & $\mathrm{n}$ & Test & $\begin{array}{c}\text { Type of CHO } \\
\text { ingested }\end{array}$ & $\begin{array}{c}\text { Amount of } \\
\text { CHO ingested }\end{array}$ & $\begin{array}{c}\text { Improve- } \\
\text { ment }\end{array}$ \\
\hline (1) Williams et al. 1988 & 15 & $2 \mathrm{~h}$ & $\mathrm{GP}+\mathrm{S}$ & $50 \mathrm{~g}$ & $\mathrm{~ns}$ \\
(2) Williams et al. 1990 & 12 & $30 \mathrm{~km}$ & $\mathrm{GP}+\mathrm{G}, \mathrm{GP}+\mathrm{F}$ & $50 \mathrm{~g}$ & $\mathrm{~ns}$ \\
& & & & & \\
(3) Millard-Stafford et al. 1992 & 8 & $40 \mathrm{~km}$ & $\mathrm{GP}+\mathrm{F}$ & $150 \mathrm{~g}$ & $10 \%$ \\
(4) Tsintzas et al. 1993 & 7 & $30 \mathrm{~km}$ & $\mathrm{GP}+\mathrm{F}$ & $50 \mathrm{~g}$ & $2.5 \%$ \\
\hline
\end{tabular}

$G=$ glucose,$G P=$ glucose polymer,$S=$ sucrose, $F=$ fructose, $n s=$ not significant 
Mechanism(s) by which CHO ingestion exerts its ergogenic effect: In most of the cycling studies, blood glucose concentration and the rate of total $\mathrm{CHO}$ oxidation gradually decrease during exercise performed without $\mathrm{CHO}$ ingestion, whereas they are maintained at higher levels when $\mathrm{CHO}$ is ingested during exercise (Coyle et al. 1983; 1986; 1991; Coggan and Coyle, 1989; Bjorkman et al. 1984; Burgess et al. 1991; Wright et al. 1991). It has been suggested that only the subjects who demonstrate a fall in blood glucose during the control trial are able to improve their endurance capacity during the $\mathrm{CHO}$ trial (Coyle et al. 1983; Bjorkman et al. 1984).

In some cycling studies, however, $\mathrm{CHO}$ ingestion during exercise improved endurance capacity and performance despite a maintained blood glucose concentration and total $\mathrm{CHO}$ oxidation rate in the control trial (Maughan et al. 1989; Murray et al. 1991). Moreover, $\mathrm{CHO}$ ingestion did not consistently increase total $\mathrm{CHO}$ oxidation rates (Erickson et al. 1987; Maughan et al. 1989). In the previous section (section 2.2) it was suggested that glycogen depletion may impair aerobic function by reducing the level of tricarboxylic acid cycle intermediates (TCAI) (Sahlin et al. 1990). Recently, it has been shown that $\mathrm{CHO}$ ingestion during cycling at $70 \% \mathrm{VO}_{2} \max$ to exhaustion delayed the onset of fatigue by enhancing the availabilty of hexose monophosphates for glycolysis which in turn attenuated the decrease in TCAI and the increase in IMP (Spencer et al. 1991).

Since similar glycogen utilization rates were observed with or without $\mathrm{CHO}$ ingestion (Coyle et al. 1986; 1991; Mitchell et al. 1989a; Hargreaves and Briggs, 1988; Fielding et al. 1985; Flyn et al. 1987), it has been suggested that $\mathrm{CHO}$ ingestion delays the onset of fatigue by maintaining euglycaemia (i.e. normal blood glucose concentration) and oxidation of blood glucose at high rates late in exercise (Coggan and Coyle, 1991).

In contrast, other studies have observed a decreased rate of muscle glycogen utilization (i.e. glycogen 'sparing' effect) as a result of $\mathrm{CHO}$ ingestion compared with water ingestion (Hargreaves et al. 1984; Bjorkman et al. 1984; Erickson et al. 1987). Glycogen sparing occurred either in the presence (Hargreaves et al. 1984; Bjorkman et al. 1984) or the absence (Erickson et al. 1987) of a declining blood glucose concentration and an increased $\mathrm{CHO}$ oxidation rate. It has been argued that the glycogen sparing effect observed in those studies could have been the effect of a higher glycogen concentration prior to the control trial 
compared with the $\mathrm{CHO}$ trial (Hargreaves et al. 1984; Erickson et al. 1987) and the longer exercise time in the $\mathrm{CHO}$ trial (Bjorkman et al. 1984) rather than a true effect of the ingested $\mathrm{CHO}$ on the glycogen utilization rate (Coggan and Coyle, 1991).

Although this might be true, it has to be pointed out that glycogenolysis follows an exponential decay pattern being very rapid during the first 15-20 min of prolonged exercise (Hermansen et al. 1967). This could be the most effective time to spare muscle glycogen; indeed, caffeine ingestion has been shown to spare muscle glycogen during the first 15 min of cycling exercise at $80 \% \mathrm{VO}_{2} \max$ (Spriet at al. 1992). If $\mathrm{CHO}$ ingestion exerts a similar effect, then apart from the pre-exercise nutritional status and the amount of the $\mathrm{CHO}$ ingested, the timing of $\mathrm{CHO}$ ingestion might play a very important role in determining the effect of $\mathrm{CHO}$ ingestion on muscle glycogen utilization.

Indeed, a close examination of the studies which did not demonstrate a glycogen sparing effect reveals that $\mathrm{CHO}$ feeding started at least 10-30 min after the initiation of exercise (Coyle et al. 1986; 1991; Mitchell et al. 1989a; Slentz et al. 1990). In the study by Fielding et al. (1985), although $\mathrm{CHO}$ ingestion started at the beginning of exercise, the amount might have been too small (10.75 g). On the other hand, in a study using a similar protocol, the ingestion of $43 \mathrm{~g}$ of $\mathrm{CHO}$ at the start of exercise resulted in glycogen sparing (Hargreaves et al. 1984). Furthermore, in most of the studies the subjects were glycogen supercompensated prior to the trials (Flynn et al. 1987; Coyle et al. 1986). It is not known to what extent high compared with normal resting glycogen concentrations could affect the utilization of glycogen when $\mathrm{CHO}$ solutions are ingested. Moreover, the type of $\mathrm{CHO}$ ingested might also be important. Bjorkman et al. (1984) observed that, unlike glucose, fructose ingestion failed to affect muscle glycogen utilization compared with water. In addition, there was no significant effect on the exercise time to exhaustion between the two treatments. Similar results were obtained by Fenn et al. (1983) during cycling to exhaustion at $70 \% \mathrm{VO}_{2}$ max.

During prolonged running exercise without $\mathrm{CHO}$ ingestion, however, blood glucose concentration does not appear to decrease to the extent it decreases during cycling exercise (Williams et al. 1990; 1992; Sasaki et al. 1987b). In fact, in 
most running studies blood glucose does not even decline (Tsintzas et al. 1993; Madsen et al. 1990; Sasaki et al. 1987a; Riley et al. 1988; Fruth and Gisolfi, 1983; Macaraeg, 1983; Wilber and Moffatt, 1992; Millard-Stafford, 1992; Wells et al. 1985; Seidman et al. 1991; Owen et al. 1986; Giles and Maclaren, 1984). In most of these studies, $\mathrm{CHO}$ ingestion resulted in increased blood glucose compared with placebo. When water or placebo is ingested $\mathrm{CHO}$ oxidation rates are maintained throughout exercise in most (Williams et al. 1990; Madsen et al. 1990; Riley et al. 1988; Fruth and Gisolfi, 1983; Wilber and Moffatt, 1992; Millard-Stafford, 1992; Sasaki et al. 1987a) but not all (Sasaki et al. 1987b; Giles and Maclaren, 1984; Owen et al. 1986) studies. Moreover, when $\mathrm{CHO}$ is ingested CHO oxidation rates are either similar (Williams et al. 1990; Fruth and Gisolfi, 1983; Riley et al. 1988; Millard-Stafford, 1992; Owen et al. 1986) or higher (Sasaki et al. 1987a; 1987b; Wilber and Moffatt, 1992; Giles and Maclaren, 1984) compared with the control. Carbohydrate ingestion has been shown to improve endurance capacity and performance in most (Macareag et al. 1983; Sasaki et al 1987a; Wilber and Moffatt, 1992; Millard-Stafford, 1992; Tsintzas et al. 1993) but not all (Riley et al. 1988; Fruth and Gisolfi, 1983; Williams et al. 1990) studies in the absence of declining blood glucose and CHO oxidation rates when placebo was ingested.

In summary, it would appear that during prolonged cycling the ingestion of solutions containing glucose, glucose polymer or sucrose, rather than fructose, would exert a positive influence on both endurance capacity and performance. There is conflicting evidence, however, with regard to running. There is also little and conflicting information regarding the exact mechanism responsible for this improvement in endurance capacity and performance. The experimental evidence suggests three possibilities: i) restoration of normal blood glucose concentration and disappearance of CNS distress which as a result of hypoglycemia could cause fatigue, ii) increased blood glucose oxidation late in exercise at a time when muscle glycogen contribution to energy metabolism is diminished, and iii) decreased rate of muscle glycogen utilization which would delay its depletion and hence the point of fatigue. 


\subsection{FACTORS AFFECTING GASTRIC EMPTYING RATE AND INTESTINAL ABSORPTION OF CHO SOLUTIONS}

As discussed in the previous section, there is a general consensus regarding the positive effect of $\mathrm{CHO}$ ingestion during exercise on endurance capacity and performance. It was also shown that oxidation of exogenous blood glucose is very important for energy metabolism during prolonged exercise, especially towards the latter stages. However, the fate of the ingested $\mathrm{CHO}$ depends on how quickly following the ingestion it empties from the stomach and is absorbed from the small intestine into the circulation before being oxidised. This section will focus on the factors affecting gastric emptying rate and intestinal absorption of ingested fluids.

Factors affecting gastric emptying rate

Many factors have been shown to influence the gastric emptying rate of a solution, namely: volume, energy content, osmolality, $\mathrm{pH}$, environmental temperature, fluid temperature, circulating hormones, time of day, stress, anger, fear and type and/or intensity of exercise (Murray, 1987; Maughan, 1991). Of these, volume and characteristics of the fluid ingested (i.e. energy content and osmolality) and type/intensity of exercise appear to be the main regulators of gastric empying rate (Noakes et al. 1991b).

Early studies (Fordtran and Saltin, 1967; Costill and Saltin, 1974) have shown that cycling exercise up to $75 \%$ of $\mathrm{VO}_{2}$ max has no significant influence on gastric emptying rate of $\mathrm{CHO}$ and electrolyte solutions compared with rest. In contrast, higher gastric emptying rates have been reported for water and various $\mathrm{CHO}$ solutions during running up to $70 \% \mathrm{VO}_{2}$ max than at rest (Neufer et al. 1986; 1989). This increase can be accounted for by runninginduced vibrations occurring in some gastro-intestinal regions (Rehrer et al. 1989). At higher ( $>70-75 \% \mathrm{VO}_{2}$ max) exercise intensities, however, a decreased rate of gastric emptying during both cycling (Costill and Saltin, 1974; Rehrer et al. 1989) and running (Sole and Noakes, 1989) compared with rest has been attributed to increased sympathetic tone and catecholamine release (Galbo et al. 1975; 1977). Recently, no difference was observed between running and cycling in the total volume of water or a $7 \% \mathrm{CHO}$ solution emptied from the stomach following repeated ingestion during a $60 \mathrm{~min}$ period (Houmard et al. 1991). Similar reșults were obtained for a $7.2 \%$ CHO solution during $80 \mathrm{~min}$ of 
either cycling or running at $70 \% \mathrm{VO}_{2}$ max; however, calculation of gastric emptying rate over 20 min periods revealed that the $7.2 \% \mathrm{CHO}$ solution emptied at a faster rate between 40 and $80 \mathrm{~min}$ of cycling compared with running (Rehrer et al. 1990).

The osmolality of the ingested solution has long been thought to be the primary determinant of gastric emptying. Costill and Saltin (1974) demonstrated that the higher the osmolality of ingested $\mathrm{CHO}$ solutions the slower the rate at which these solutions leave the stomach. The inverse relationship between the osmolality of an ingested fluid and its gastric emptying rate has also been demonstrated by Coyle et al. (1978).

Since glucose solutions have much higher osmolality than glucose polymer solutions at equal $\mathrm{CHO}$ concentrations (i.e. same energy density), Foster et al. (1980) compared the gastric emptying characteristics of glucose and glucose polymer solutions of different concentrations $(5,10,20$ and $40 \%$ ) at rest. They found that at low $\mathrm{CHO}$ concentrations ( $\leq 5 \%)$, the glucose polymer produced a significantly faster gastric emptying rate. However, no significant differences in gastric emptying were found for the more concentrated solutions (10, 20 and 40\%). More recent studies, however, have shown that osmolality may not be as important in regulating gastric emptying rate as previously thought. Indeed, little, if any physiologically meaningful, difference exists in the gastric emptying rate of isoenergetic solutions of different osmolality (i.e. glucose polymer vs glucose solutions) (Naveri et al. 1989; Rehrer et al. 1990; Owen et al. 1986; Vist and Maughan et al. 1993). This is especially true at $\mathrm{CHO}$ concentrations up to $10 \%$. At higher concentrations, however, glucose polymer solutions tend to empty faster than glucose solutions (Vist and Maughan, 1993; Sole and Noakes, 1989; Rehrer et al. 1990).

It has also been shown that as the energy content of an ingested solution increases, the gastric emptying rate decreases (Costill and Saltin, 1974; Hunt and Stubbs, 1975). Water has been shown to produce faster gastric emptying rates than $\mathrm{CHO}$ solutions at concentrations above 2.5\% (Costill and Saltin, 1974; Neufer et al. 1986; Vist and Maughan, 1993). As a consequence, it has been recommended that fluids used for replacement during exercise should contain no more than $2.5 \% \mathrm{CHO}$ (Costill and Miller, 1980). The finding that isoenergetic solutions of different osmolality empty from the stomach at the same rate coupled with the observation that isoosmotic solutions of different energy content empty at a different rate (i.e. the higher the energy density the 
slower the emtying rate) (Hunt and Stubbs, 1975; Vist and Maughan, 1993) has led to the suggestion that the energy density of a $\mathrm{CHO}$ solution is the prime determinant of gastric emptying rate (Vist and Maughan, 1993; Murray, 1987). It was also pointed out that the overriding influence of the ingested solution's energy content is not apparent because it is often proportional to fluid's osmolality (Murray,1987).

Most of the studies, however, have measured gastric content at the end of an exercise bout, usually after $1-2 \mathrm{~h}$, and thus it was possible to determine average but not instantaneous rates of gastric emptying rate. A modification of the double sampling technique of George (Beckers et al. 1988) enables multiple measurement of net gastric emptying over a certain period of time (Rehrer et al. 1989; 1990). Using this technique it was shown that gastric emptying follows an exponential time course with the fastest rate being immediately after the ingestion of the fluid. This was true following either the ingestion of a single bolus or repeated drinking of fluid during the test period (Rehrer et al. 1989; 1990). However, gastric emptying rate was faster when the test fluid was ingested repeatedly. It was suggested therefore that gastric volume is a very important regulator of gastric emptying rate (Noakes et al. 1991b). Indeed, a polynomial relationship has been shown to exist between the volume of fluid ingested and the volume of fluid emptied from the stomach (Mitchell and Voss, 1991). It would appear that the effect of volume on gastric emptying may be related to pressure exerted by a large fluid volume against the stomach's wall (Rehrer et al. 1989) and thus it is of transient nature.

A repeated drinking pattern, however, would maintain a higher average volume of fluid in the stomach and, in that way, would alter the gastric emptying characteristics of the fluids ingested. Indeed, solutions containing even up to $8 \%$ CHO could be used during exercise without impairing gastric emptying rate compared with water when administered in serial feedings every 15-20 minutes (Mitchell et al. 1988; 1989b; Ryan et al. 1989; Rehrer et al. 1990; Houmard et al. 1991). When the volume of these serial feedings is kept medium (100-200 $\mathrm{ml}$ ) then the average gastric emptying rate of water or $\mathrm{CHO}$ solutions ingested has been shown to range between $6.6-10.9 \mathrm{ml} . \mathrm{min}^{-1}$ (Fordtran and Saltin, 1967; Owen et al. 1986; Mitchell et al. 1988; 1989b; Houmard et al. 1991). On the other hand, higher average gastric emptying rates (17-19 ml.min $\left.{ }^{-1}\right)$ were reported following ingestion of multiple feedings of higher volume (350-400 ml) (Ryan et al. 1989; Mitchell and Voss, 1991). Even 
when smaller volumes of fluid $(150-200 \mathrm{ml})$ were ingested following an initial large bolus (600-750 ml), an average gastric emptying rate between 14.2-30.1 ml.min ${ }^{-1}$ reported for $\mathrm{CHO}$ solutions of $6-10 \%$ concentration (Rehrer et al. 1990; Duchman et al. 1990).

\section{Factors affecting intestinal absorption}

Although, gastric emptying rate has been considered as the main limiting step in the oxidation of exogenous glucose by the working muscles (Costill and Saltin, 1974), recent evidence suggests that it might not necessarily be the sole determinant of the actual rate at which the $\mathrm{CHO}$ content of any fluid enters the circulation. The more concentrated solutions will empty slower but the amount of glucose delivered to the small intestine will be greater than that of less concentrated solutions (Rehrer et al. 1992; Vist and Maughan, 1993).

As the amount of $\mathrm{CHO}$ delivered to the small intestine appears to be more than the amount of exogenous glucose oxidised by the muscles (Rehrer et al. 1992), it has been suggested that its absorption rather than gastric emptying rate determines its delivery to the muscles (Hawley et al. 1992). However, absorption rate of glucose has been shown to increase with increasing delivery of glucose into small intestine (Rehrer et al. 1992) suggesting that intestinal absorption might not be a limiting factor in determining the rate of exogenous glucose oxidation. Indeed, it was shown that the latter was relatively the same after ingesting a $4.5 \%$ and a $17 \%$ CHO solutions despite a 3.3 times faster absorption rate of $\mathrm{CHO}$ from the latter compared with former solution (Rehrer et al. 1992). Thus, although $\mathrm{CHO}$ solutions may have slower gastric emptying rate than plain water, their absorption in the small intestine is higher due to the stimulating effect of glucose and sodium (Murray, 1987).

Absorption of carbohydrate, electrolytes and water occurs mainly in the duodenum and jejunum sections of the small intestine. Absorption of water is a passive process which occurs as a result of osmotic gradient developed between the intestinal lumen and the interstitial space by the movement of glucose, sodium and other nutrients. Glucose and galactose compete for the same transport. They are both transported actively by a means of a sodiumdependent protein carrier located in the luminal and lateral borders of the small intestine epithelium cells. Some glucose, water and sodium can also cross the brush border passively via a paracellular pathway (between the cells 
as opposed to through the cells), especially when luminal glucose concentrations are above those that saturate the membrane carriers (Pappenheimer, 1990). Fructose is transported by a different protein-carrier which is not sodium-dependent, and is less rapidly absorbed than glucose (Fordtran, 1975). As a result fructose ingestion leads to smaller absorption of water and sodium, and when ingested in large amounts produces gastrointestinal distress. Sucrose is rapidly hydrolysed to glucose and fructose and it has been shown that it is equally effective in stimulating glucose absorption (Jones et al. 1987). However, there is some evidence that sucrose solutions might stimulate less water absorption than free glucose solutions and water (Wheeler and Banwell, 1986). It has also been shown that glucose absorption is greater from maltose, maltotriose and 1-4, but not 1-6, linked glucose oligosaccharides (less than 10 units) solutions compared with free glucose (Jones et al. 1983; 1987).

In general, slightly hypotonic solutions compared with plasma stimulate higher intestinal fluid absorption and similar electrolyte absorption compared with isotonic or hypertonic solutions (Hunt et al. 1988; Leiper and Maughan, 1986a; 1986b). Hypertonic solutions will result in secretion of water into the small intestine due to an increased osmolality which will create a favorable osmotic gradient for water secretion (Maughan, 1991).

It is important, however, to distinguish between the gastric emptying rate and intestinal absorption of $\mathrm{CHO}$ and of fluid (Noakes et al. 1991b). Concentrated $\mathrm{CHO}$ solutions might result in greater absorption of $\mathrm{CHO}$ into the bloodstream and increased availability of glucose for the working muscles at the expense of sufficient fluid delivery. Segmental perfusion of the small intestine (Cooper et al. 1966) and accumulation in the plasma of isotopic tracers like deuterium oxide $\left(\mathrm{D}_{2} \mathrm{O}\right)$ after ingestion of $\mathrm{D}_{2} \mathrm{O}$-labeled solutions (Davis et al. 1987) have been used as direct indexes of entry of the fluid ingested into the general circulation. Using the latter technique a reduced fluid absorption by the blood was shown during exercise of different intensities compared with rest (Maughan et al. 1990). In contrast, using segmental perfusion of the small intestine no difference was observed in intestinal absorption of water or $6 \% \mathrm{CHO}$ solution between rest and exercise (Fordtran and Saltin, 1967). 
This is not the only disagreement between the two techniques; the use of the $\mathrm{D}_{2} \mathrm{O}$ technique during exercise resulted in similar amount of fluid absorbed into the circulation from water and $\mathrm{CHO}$ solutions of $6 \%, 8 \%$ and $10 \%$ containing simple sugars or maltodextrins (Davis et al. 1990); a 12\%, 15\% and $40 \%$ glucose solution, however, resulted in significantly less fluid absorption compared with water (Davis et al. 1987; 1988b). In contrast, Gisolfi et al. (1990) studied the rate of fluid appearance in the plasma following consumption of water, a $6 \%$ and $10 \%$ CHO solution using both techniques (i.e. segmental perfusion and accumulation of $\mathrm{D}_{2} \mathrm{O}$ in plasma). They found that water and the $6 \% \mathrm{CHO}$ solution produced net fluid absorption whereas the $10 \% \mathrm{CHO}$ solution produced net fluid secretion; the $6 \%$ solution was more effective than water. In contrast, the $\mathrm{D}_{2} \mathrm{O}$ accumulation in the plasma was similar for all three solutions. It was concluded that the latter technique reflects the unidirectional movement of fluid and therefore is not a valid measure of net fluid movement across the intestinal wall (Gisolfi et al. 1990).

Nevertheless, taken together these results seems to suggest that the ingestion of $\mathrm{CHO}$ solutions of low concentrations ... would provide the muscles with the neccessary energy in the form of glucose without compromising the availability of fluid. This is very important since the aims of ingesting a $\mathrm{CHO}$ solution during prolonged exercise are to provide an alternative source of energy to the muscles and offset dehydration and the associated decrease in performance. 


\subsection{INFLUENCE OF DEHYDRATION AND FLUID INGESTION ON TEMPERATURE REGULATION}

During prolonged intense exercise the increased energy requirements are met by an increased metabolic rate which results in increased heat production. Vaporization of sweat is the main mechanism for heat dissipation during prolonged exercise in thermoneutral and hot environments. During prolonged physical activity sweat rates between 1.0 and $3.71 . \mathrm{h}^{-1}$ have been reported (Murray, 1987; Armstrong et al. 1986; Herbert, 1983; Sawka, 1992). The higher sweat rates were achieved in hot environments. As a result of such a marked dehydration body weight may be reduced by up to $8 \%$ during a marathon race (Costill and Miller, 1980). It has been shown that a decrease in body weight of more than $2 \%$ by exercise-induced sweating impairs cardiovascular and thermoregulatory functions which may compromise endurance performance (Costill and Miller, 1980; Saltin et al. 1972). Recently, it was suggested that fatigue during prolonged exercise at $60-70 \% \mathrm{VO}_{2} \max$ performed in the heat is the result of impaired motor centre function or mental drive for motor performance due to high core temperature (Nielsen et al. 1990).

Severe losses of intracellular and extracellular fluids during prolonged heavy exercise are associated with an increase in the threshold core temperature for initiation of sweating and, thus, decreased sweat rate (Fortney et al. 1984). In fact, a systematic reduction of sweat rate has been observed for a given core temperature with increased levels of dehydration (Sawka et al. 1985). This will minimize heat loss and raise core temperature (Claremont et al. 1976) towards high levels which will cause an early heat fatigue during prolonged exercise (Saltin et al. 1972). Although a linear relationship seems to exist between elevation in core temperature and degree of dehydration (Sawka, 1992), it has been suggested that metabolic rate rather than dehydration is the most important factor determining the rise in rectal temperature during prolonged exercise (Noakes et al. 1991a; Maughan et al. 1985).

It has been suggested that a higher plasma osmolality as a result of dehydration is a very important stimulus for the reduced sweat rate with increasing core temperature (Sawka, 1992). A reduced blood volume (i.e. hypovolaemia) accompanied by a normal osmolality has also been shown to increase core temperature and reduce skin blood flow (Fortney et al. 1981). 
Consequently, it has been suggested that losses of intracellular and extracellular fluids rather than decreased plasma volume per se could be the actual cause of many of the adverse effects of dehydration on the ability to perform prolonged exercise (Lamb and Brodowicz, 1986). It has also been shown that severe dehydration is associated with decreased stroke volume (Murray, 1987) and increased heart rate (Claremont et al. 1976). More recent evidence has shown that blood flow to the working muscles was not reduced in the presence of high core and muscle temperatures (Nielsen et al. 1990). Taken together these findings would suggest that during exercise-induced dehydration, sweat rate and skin blood flow are reduced in order to conserve blood volume and maintain blood flow to the working muscles (Sawka, 1992).

Therefore, the loss of substantial intracellular and extracellular fluid volumes that normally results from prolonged heavy sweating, will increase the stress placed on the cardiovascular system's responsibilities of oxygen and heat transport. Unless a substantial portion of sweat loss is replaced by drinking fluids during prolonged exercise, dehydration and the associated reduction in heat loss responses will have detrimental effects on the ability to perform such an exercise (Murray, 1987).

Indeed, fluid replacement during prolonged exercise has been shown to prevent the decrease in stroke volume and cardiac output and attenuate the decrease in plasma volume and the increase in rectal temperature compared with no fluid consumption (Hamilton et al. 1991; Barr et al. 1991). It seems unlikely, however, that this effect of fluid ingestion is mediated by the attenuated decrease in blood volume since expansion of blood volume to the level observed during exercise with fluid consumption does not prevent the increase in rectal temperature (Montain and Coyle, 1992). Moreover, compared with fluid replacement, blood volume expansion failed to attenuate the decrease in skin blood flow observed with no fluid ingestion. It was suggested that fluid ingestion reduces hyperthermia by increasing skin blood flow and limiting an increase in serum osmolality (Montain and Coyle, 1992).

It has been suggested that in order to prevent dehydration and its effects on aerobic capacity fluid replacement during exercise should be equal to the body weight loss or in other words match the rate of sweat loss (Coyle and 
Montain, 1992a). On the other hand, it has been proposed that the rate of fluid intake should be less than the rate of sweat loss due to the fact that metabolic water and water stored with glycogen is released during exercise, and thus should not be taken into account when estimating the rate of fluid intake. On that basis, it has been recommended that an average fluid intake of $0.51 . \mathrm{h}^{-1}$ is an adequate rate of fluid replacement during prolonged exercise (Noakes et al. 1988). This rate of fluid intake appears to be the ad libitum fluid intake during prolonged exercise such as marathon running.

During exercise, thirst does not seem to be a sufficient stimulus for maintaining body water. Therefore, drinking ad libitum will give rise to the so called phenomenon of 'involuntary dehydration' (Greenleaf, 1992). Recently, it was shown that core temperature, heart rate and cardiac output were directly related to the rate of fluid ingestion; the higher the rate of fluid intake the greater the attenuation in the increase in core temperature and heart rate and in the decrease in cardiac output (Coyle and Montain, 1992b). Therefore, it seems that at least in theory the rate of fluid replacement during exercise should match the rate of body weight loss. However, factors such as the mode and intensity of exercise, the physiological and biochemical characteristics of the athlete and the occurrence of gastrointestinal discomfort should also be taken into account. 


\subsection{SUMMARY}

Carbohydrate is the most important energy source during prolonged heavy exercise. Although fatigue during this type of exercise could be either central or peripheral in origin, there is strong evidence accumulated over the last 25 years to suggest that fatigue coincides with depletion of glycogen in the working skeletal muscles. Under certain conditions, however, prolonged exercise could lead to fatigue well before glycogen in the working muscles becomes depleted due to dehydration and hyperthermia. Therefore, the aim of ingesting any $\mathrm{CHO}$ solution during exercise is to delay the onset of fatigue by providing an alternative fuel source which is immediately usable by the working muscles and replacing the body fluid lost through sweating. The experimental evidence suggests that during prolonged cycling the ingestion of solutions containing glucose, glucose polymer or sucrose, rather than fructose, would exert a positive influence on both endurance capacity and performance. There is conflicting evidence, however, with regard to running. Fluid ingestion has also been shown to attenuate the dehydration and hyperthermia associated with prolonged exercise. In general, it appears that the ingestion of $\mathrm{CHO}$ solutions of concentrations up to $10 \%$ would provide the working muscles with the neccessary energy in the form of glucose without compromising the availability of fluid. 


\section{CHAPTER 3}

\section{GENERAL METHODS}

\subsection{INTRODUCTION}

In this thesis, two different experimental models were used to investigate the effect of carbohydrate ingestion on endurance capacity and endurance performance during treadmill running exercise. Endurance capacity was assessed by the time taken to reach exhaustion while running at a constant pace (Studies 2 and 4). Endurance performance was also assessed by the time taken to complete a certain pre-determined distance (Study 1). In study 3, a slightly different model was used. Since the main aim of that study was to investigate the metabolic responses to carbohydrate and water ingestion during exercise of equal duration, a non-fatiguing bout of running exercise (i.e. $60 \mathrm{~min})$ at constant pace $\left(70 \% \mathrm{VO}_{2} \mathrm{max}\right)$ was employed.

This chapter has been divided into three sections. The first section describes the equipment and testing procedures used during the administration of all the preliminary and main experimental exercise tests. The second and third sections deal with the procedures associated with collection, treatment, storage and analysis of blood and muscle specimens, respectively. 


\subsection{EXPERIMENTAL TESTING PROCEDURES}

\section{Equipment}

All experimental tests administered in this thesis were conducted in the laboratory under neutral environmental conditions (18-22 $\left.{ }^{\circ} \mathrm{C}\right)$. Environmental dry and wet bulb air temperatures were measured and adjusted accordingly throughout the tests using a whirling hydrometer (Brannan). Dry and wet temperatures were subsequently used to calculate the relative humidity by an appropriate conversion scale.

A motorised treadmill (Woodway, model ELG 2) was used in all tests. Running speed and distance covered were recorded continuously throughout the runs by a microcomputer ( $B B C$ Master Series) interfaced with the treadmill.

The collection of the expired air samples was made using the Douglas bag method. The subjects breathed into a low resistance respiratory valve which was connected to a 150 litre Douglas bag by lightweight smooth bore tubing (Falconia $\mathrm{Ltd}$ ). Oxygen $\left(\mathrm{O}_{2}\right)$ and carbon dioxide $\left(\mathrm{CO}_{2}\right)$ percentages of those samples were determined using a paramagnetic oxygen analyser (Sybron Taylor, model 570A) and an infrared carbon dioxide analyser (Lira, model 303), respectively. A dry gas meter (Harvard Ltd) and a thermometer (Edale Instruments Ltd, model $\mathrm{C}$ ) have been used to measure the volume and the temperature of each expired air sample, respectively. Using the temperature of the expired air, the barometric pressure obtained from a barometer (Griffen and George Ltd), and the Haldane transformation formula, all the gas volumes obtained were corrected to STPD. This allowed the calculation of oxygen uptake $\left(\mathrm{VO}_{2}\right)$, carbon dioxide $\left(\mathrm{VCO}_{2}\right)$ production, minute ventilation (VE), and respiratory exchange ratio (RER).

In studies 1 and 2, heart rate was continuously recorded from four 'Red dot' silver chloride self-adhesive chest electrodes connected to a heart rate monitor (Rigel 302). In studies 3 and 4, heart rate was monitored throughout the tests by short range telemetry (Polar Electro sports testers PE 3000).

In studies 1 and 2, skin and rectal temperatures were recorded throughout the main experimental tests. To do this, small temperature-sensitive pads were 
placed on the subject's calf, thigh, arm and chest, and a rectal probe was inserted to a depth of $10 \mathrm{~cm}$. The 4 thermistors and rectal probe were connected to a thermometer (Edale Instruments Ltd, model C) for continuous monitoring and recording of skin and rectal temperatures respectively. Mean skin temperature (Tsk) was calculated using the following equation: $T s k=[0.3$ $*($ Tarm + Tchest $)+0.2 *($ Tcalf + Tthigh $)]$.

In all studies nude body weight $(\mathrm{kg})$ was obtained using a balance accurate to two decimal places (Avery $3306 \mathrm{ABV}$ ). Body weight measurements were performed at rest, after the subject emptied his bladder, and immediately following the completion of the test, after the removal of the sweat from the skin. Body height of all subjects was obtained using a stadiometer (Holtain Ltd).

\section{Preliminary measurements}

Familiarization: All subjects who volunteered to take part in these studies were familiarised with testing procedures. This consisted of a number of practice sessions until subjects became fully confident of running on a motorised treadmill. The subjects also became familiarised with collection of expired air samples and ingestion of fluid while running on the treadmill.

In all studies, a series of preliminary tests was conducted prior to the main experimental trials. In studies 1 and 2, three preliminary tests were carried out in order to determine: $i$ / the relationship between oxygen uptake and running speed (running economy), ii/ the maximum oxygen uptake ( $\mathrm{VO}_{2}$ max) and iii/ the running speeds equivalent to blood lactate concentrations [La] of 2 and 4 mmol.1-1 for each individual. In studies 3 and 4 , the first two preliminary tests were conducted only.

Determination of running economy: The duration of this continuous submaximal test was 16 minutes. The initial speed was set between 2.5 and 3.5 $\mathrm{m} . \mathrm{s}^{-1}$ depending on the subject's training status. The speed increased every four minutes by $0.5 \mathrm{~m} . \mathrm{s}^{-1}$. Treadmill speed and distance covered, were monitored and recorded precisely by a microcomputer (BBC Master Series) connected with the treadmill. The inclination of the treadmill was kept at zero throughout the test. Expired air samples were collected during the last minute of each four-minute period. Heart rate was also monitored throughout the 
test. Using the data derived from this test a regression analysis relating running speed and oxygen cost was produced for each subject.

Determination of maximal oxygen uptake ( $\mathrm{VO}_{2}$ max): The maximal oxygen uptake of each subject was determined during uphill treadmill running using a continuous incremental test to exhaustion (Taylor et al. 1955). The test was divided into three-minute periods. The treadmill speed was kept constant throughout the test and the inclination of the treadmill increased by $2.5 \%$ every three minutes from an initial 3.5\%. Prior to the test a self selected warmup was undertaken by all subjects. Expired air samples were taken between 1:45-2:45 minutes of every three-minute period. The final collection of expired air was made during the last minute of the test after the subject indicated by raising his hand that he was able to continue for only one more minute. Continuous verbal encouragement was given to each subject during the test. Heart rate was monitored throughout the test and perceived rate of exertion was recorded every three minutes. The $\mathrm{VO}_{2}$ value obtained during the last expired air sample was taken as the $\mathrm{VO}_{2}$ max value of this individual.

Determination of running speeds equivalent to [La] of 2 and 4 mmol.1-1 : Based on the $\mathrm{VO}_{2}$ max value as determined by the previous test, $60 \%, 70 \%$, $80 \%$, and $90 \%$ of $\mathrm{VO}_{2}$ max were then calculated. Using this data and the regression equation derived from the first test, four submaximal running speeds corresponding to $60 \%, 70 \%, 80 \%$, and $90 \%$ of $\mathrm{VO}_{2}$ max were predicted. During this test the subjects ran continuously on a level treadmill at these submaximal speeds four minutes at each speed. Expired air samples were taken between 2:45 and 3:45 minute of each four-minute period. Heart rate was also monitored throughout the test. In order to determine blood lactate concentrations, duplicate capillary blood samples were obtained from the subject's thumb immediately after each expired air collection. A resting preexercise capillary blood sample was also taken from a pre-warmed hand. The data obtained from this test enabled the running speed equivalent to blood lactate concentrations of 2 and $4 \mathrm{mmol} \cdot \mathrm{l}^{-1}$ to be calculated.

In all studies, following the completion of the preliminary tests, a treadmill run (60-90 min) at $70 \% \mathrm{VO}_{2}$ max was also performed by each subject a week before the first experimental run. This run was undertaken to ensure that the subjects were fully familiarized with prolonged treadmill running and the procedures which were to be used. 
Nutritional control: The experimental trials in all studies took place following a 12-16 hour overnight fast to ensure an empty stomach and minimize the effect of a previous meal on the gastric emptying rate of the test solutions. Also, to control the effect of nutritional status on the results of each study, the subjects consumed a normal standardised diet for two days (Studies 1 and 2) and three days (Studies 3 and 4 ) before each trial.

In studies 1 and 2, each subject's normal food intake was assessed a few weeks before the first experimental run by a means of a 7-day weighed food intake analysis (Paul and Southgate, 1978). In study 2, all the meals for the two days prior to each trial were prepared and consumed in the laboratory. In studies 3 and 4 , the subjects recorded their normal diet for three days preceding the first trial. They replicated the same diet for the same period of time before the second trial.

In all studies, the subjects followed the same training schedule for one week before each trial. The runners refrained from heavy exercise for two days preceding each trial.

\section{Experimental protocol}

In study 1 , endurance performance was measured as the time taken to complete a full marathon distance $(42.2 \mathrm{~km})$. The subjects were required to cover the distance as quickly as possible. In order to ensure a maximum endurance performance they were able to adjust their running pace using a hand-held micro-switch. Running speed and distance covered were recorded continuously throughout the runs by a microcomputer (BBC Master Series) interfaced with the treadmill. In studies 2 and 4 , endurance capacity was measured as the time taken to reach exhaustion during running at a constant pace which would elicit $70 \%$ of their respective $\mathrm{VO}_{2}$ max. Exhaustion was defined as that point in time when the subjects were unable to maintain the pace of the treadmill. In study 3, a $60 \mathrm{~min}$ run at $70 \% \mathrm{VO}_{2}$ max was employed to study metabolic and physiological responses to $\mathrm{CHO}$ and water ingestion during exercise of equal duration.

In studies 1 and 2, the subjects were required to complete 3 experimental trials. In one of the trials water was ingested during exercise (control trial), whereas a 
$5.5 \%$ and a $6.9 \%$ carbohydrate-electrolyte solutions were ingested during the other two trials (these are the concentration values supplied by the manufacturers). In studies 3 and 4, the subjects completed two trials; on one occasion water (study 3) or placebo (study 4) was ingested (control trial), whereas on the other occasion a 5.5\% carbohydrate-electrolyte solution was given. The same $5.5 \% \mathrm{CHO}$ drink (A) was used in all studies. It should be noted, however, that the composition of the $6.9 \% \mathrm{CHO}$ solution (C) used in study 2 was slightly different to the one used in study 1 (B). The composition of all the carbohydrate-electrolyte solutions used in the studies of this thesis is shown in Table 3.1. The main reason for using these particular $\mathrm{CHO}$ solutions was that in taste trials on several beverages they were chosen as the most palatable at the end of several training sessions by a large group of athletes. In addition to this, the $6.9 \% \mathrm{CHO}$ solution was chosen because it is a commercially available sports drink (Lucozade Sport, SmithKline Beecham) widely used in the UK for fluid replacement during exercise.

The drinking pattern employed in each study is described in the individual chapters. Plastic volumetric syringes were used by the runners for fluid ingestion to avoid any spillage and ensure that the right amount was consumed. Fluid ingestion did not interrupt running. Following the completion of each trial, the subject completed a questionnaire about the taste and palatability of the ingested solution, the occurrence of gastrointestinal disturbances, hypoglycaemic symptoms and muscle cramps, pain and soreness.

Table 3.1: Composition of the solutions used in the studies of this thesis ( $A=5.5 \%$ CHO solution; $B=6.9 \%$ CHO solution used in study $1 ; \mathrm{C}=6.9 \% \mathrm{CHO}$ solution used in study 2)

$\begin{array}{lrrr} & \text { A } & \text { B } & \text { C } \\ \text { Dextrose (g.l-1) } & 1.7 & 1.9 & 2.2 \\ \text { Fructose (g.l-1) } & 1.1 & 1.9 & 0.5 \\ \text { Maltose (g.l-1) } & 0.6 & 0.5 & 0.5 \\ \text { Higher saccharides (g.l-1) } & 1.9 & 2.3 & 3.1 \\ \text { Sodium (mg.100-1 ml) } & 61 & 55 & 55 \\ \text { Potassium (mg.100-1 ml) } & 10 & 20 & 20 \\ \text { Osmolality (mOsm.kg-1) } & 280 & 260 & 260 \\ \text { Energy value (KJ.100-1 ml) } & 90 & 110 & 110\end{array}$




\section{SAMPLES}

Sample collection: In studies 1 and 2, venous blood samples were obtained from a forearm vein using an indwelling catheter (butterfly, $21 \mathrm{G}$ ), which was inserted without the application of local anaesthetic. In studies 3 and 4 , venous blood samples were obtained from an ante-cubital vein using an indwelling catheter (venflon, 16-18 G), which was inserted under local anaesthetic $(0.5 \mathrm{ml}$ of $1 \%$ lignocaine). In both cases, the catheter was inserted following $15-20 \mathrm{~min}$ of passive rest on an examination couch, and was kept patent by infusion of sterile heparinised saline (10 units per $\mathrm{ml}$ ). A $10 \mathrm{ml}$ resting venous blood sample was obtained either when the subject was still lying on the couch (study 1) or following 15-20 min in the standing position (studies 2, 3 and 4). During exercise, $5 \mathrm{ml}$ venous blood samples were taken at regular intervals without interrupting running. The post-exercise blood sample $(10 \mathrm{ml})$ was obtained either immediately after the run (study 1 and 2 ) or during the last min of the run (studies 3 and 4).

Methodological considerations on estimating changes in plasma volume: In all studies, changes in plasma volume were estimated using the changes in haemoglobin concentrations and haematocrit values according to the formula by Dill and Costill (1974). These changes in plasma volume were measured in order to assess, in conjunction with changes in heart rate response, the effects of dehydration and/or rehydration on cardiovascular function. In studies 3 and 4 , the $10 \mathrm{~min}$ into exercise blood sample rather than the resting sample was taken as the baseline for the calculation of the change in plasma volume during the run.

The reason for this is that a change in the plasma volume occurs as a result of the transition from rest to exercise. This change is not related to body water loss observed following prolonged exercise. Depending on whether this change in plasma volume is haemoconcentration (which is usually the case) or haemodilution, taking the resting sample as a baseline would either overestimate or underestimate the change in plasma volume due to body water loss. Indeed, on average a $5.4 \pm 1.9 \%$ (mean $\pm S E M, n=15$ ) decrease in plasma volume (haemoconcentration) was observed during the first $10 \mathrm{~min}$ of exercise in studies 3 and 4 . Therefore, to minimize the effects of factors like transition from rest to exercise and postural changes on plasma volume 
change, the $10 \mathrm{~min}$ blood sample and the blood sample obtained during the last minute of the run were taken as the baseline and the end-point, respectively, for the calculation of plasma volume changes in these studies.

Treatment, storage and analysis of blood samples: In all studies, venous blood samples were collected into lithium-heparinised tubes except for $2 \mathrm{ml}$ of blood which was left to clot to obtain serum (studies 2, 3 and 4), and $1 \mathrm{ml}$ of blood which was immediatelly placed into calcium-heparinised tubes (50 units per tube) and centrifuged for $5 \mathrm{~min}$ at $12000 \mathrm{rpm}$ (studies 1 and 2). The plasma obtained was stored at $-70^{\circ} \mathrm{C}$ before being analysed, at a later date, for sodium and potassium concentrations by flame photometry (Corning, model 435). However, part of this plasma was analysed on the same day of collection for ammonia concentration using a commercially available kit (Boeringher Mannheim). A pilot study on the effect of freezing and storage conditions on ammonia concentration of plasma specimens showed that plasma samples could be stored at $-70^{\circ} \mathrm{C}$ up to $48 \mathrm{hrs}$ without any significant change in ammonia concentration. Moreover, there was no difference in ammonia concentration between snap-frozen samples and samples stored at $-70^{\circ} \mathrm{C}$.

Duplicate $20 \mu \mathrm{l}$ aliquots of the whole venous blood were deproteinised in 200 $\mu \mathrm{l}$ of perchloric acid $(2.5 \%)$ and stored at $-20^{\circ} \mathrm{C}$ before being analysed for blood lactate concentration using a modification of the method described by Maughan (1982), and for blood glucose using the glucose oxidase method as described by Werner et al. (1970). Another $40 \mu \mathrm{l}(2 \times 20 \mu \mathrm{l})$ of the whole venous blood was used for the determination in duplicate of haemoglobin concentration by the cyanomethaemoglobin method (Boeringher Mannheim $\mathrm{GmbH}$ test- combination). Triplicate $20 \mu \mathrm{l}$ aliquots of blood were analyzed for haematocrit values using a micro-centrifuge (Hawksley Ltd). As mentioned previously, percentage changes in plasma volume were estimated from the haemoglobin and haematocrit values as described by Dill and Costill (1974).

The remainder of the whole venous blood was centrifuged for $15 \mathrm{~min}$ at 6000 rpm at temperature of between $3^{\circ}$ and $4^{\circ} \mathrm{C}$, and the plasma obtained was stored at $-20^{\circ} \mathrm{C}$ before being analysed at a later date. In study 1, this plasma was analysed for: FFA (Chromy et al. 1977); glycerol (Laurell and Tibbling, 1966); urea (Boeringher Mannheim kit); creatine kinase (fluorometrically using NADPH standards and Boeringher Mannheim reagents); insulin (Soeldner and Sloane,1965); cortisol (Farmos Diagnostica Orion Corporation, Turku, 
Finland 125 direct radioimmunoassay kit); growth hormone (solid phase twosite immunoradiometric assay, Netria laboratories, London, UK); and adrenaline and nor-adrenaline concentrations (Forster et al. 1991).

In studies 2, 3 and 4, the plasma obtained was analysed at a later date for FFA using a commercially available kit (Wako) and glycerol (Laurell and Tibbling, 1966) concentrations. In study 2 , part of the plasma was also stored at $-70^{\circ} \mathrm{C}$ before being analysed for adrenaline and nor-adrenaline concentrations.

In studies 2, 3 and 4, serum was also obtained by centrifugation for $15 \mathrm{~min}$ at $6000 \mathrm{rpm}$ and stored at $-70^{\circ} \mathrm{C}$. In study 2 , the serum obtained was analysed for insulin, cortisol and growth hormone concentrations (see study 1). In study 3 , serum was analysed for insulin concentration only, whereas in study 4 , serum was analysed for insulin, sodium and potassium concentrations.

The coefficient of variation $\{($ Standard Deviation/mean $) * 100\}$ of the blood, plasma and serum assays is shown in Table 3.2.

Table 3.2: Coefficient of variation of blood, plasma and serum metabolite assays $(\mathrm{n}=15)$.

$\begin{array}{ll}\text { Blood lactate } & 1.8 \% \\ \text { Blood glucose } & 1.5 \% \\ \text { Plasma and serum sodium } & 1.0 \% \\ \text { Plasma and serum potassium } & 0.9 \% \\ \text { Plasma ammonia } & 2.0 \% \\ \text { Plasma FFA } & 1.0 \% \\ \text { Plasma glycerol } & 3.1 \% \\ \text { Plasma urea } & 1.1 \% \\ \text { Plasma Creatine kinase } & 2.9 \% \\ \text { Plasma adrenaline } & 10.0 \% \\ \text { Plasma nor-adrenaline } & 3.0 \% \\ \text { Plasma and serum insulin } & 5.6 \% \\ \text { Plasma and serum cortisol } & 3.6 \% \\ \text { Plasma and serum Growth hormone } & 4.6 \%\end{array}$




\section{Collection of muscle samples}

Muscle samples were obtained from vastus lateralis by a percutaneous needle biopsy technique (Bergstrom, 1962) with suction being applied. All samples were taken under local anaesthetic (2-3 $\mathrm{ml}$ of $1 \%$ lignocaine), and their size ranged between 30 and $100 \mathrm{mg}$ wet weight. The vastus lateralis muscle was selected because, apart from being easily accesible, it was assumed to be heavily involved during treadmill running exercise. It is generally believed that gastrocnemius rather than quadriceps muscle is more involved during running exercise (Costill et al. 1974). However, a difference may exist between road and treadmill running in the instantaneous reaction force vectors due to the fact that, unlike the surface of the road, the belt of the treadmill is moving in the opposite direction to the runners direction. If the moments generated by the gastrocnemius muscle are different in the two situations, then a difference in the contribution of this muscle is also expected. The difference between road and treadmill running would explain the big difference in the gastrocnemius glycogen concentration observed after a road marathon race (Sherman et al. 1981) and a treadmill run to exhaustion (Madsen et al. 1990) (108 mmol. $\mathrm{kg} \mathrm{dm}^{-1}$ vs $434 \mathrm{mmol}^{\mathrm{kg} \mathrm{dm}} \mathrm{dm}^{-1}$ respectively). Average glycogen utilization rate was also much higher during the road running compared with treadmill running $\left(4.2 \mathrm{mmol} . \mathrm{kg} \mathrm{dm}^{-1} \cdot \mathrm{min}^{-1}\right.$ vs $2.2 \mathrm{mmol} . \mathrm{kg} \mathrm{dm}^{-1} \cdot \mathrm{min}^{-1}$ respectively). It should be noted that in both studies a significant amount of glycogen was still present in the muscles of the runners at the end of exercise.

It has also been suggested that following exhaustive road running no real difference exists in the glycogen depletion pattern of the gastrocnemius and the lateral portion of quadriceps muscles (Karlsson and Saltin, 1971). Moreover, anectodal evidence from our laboratory suggested that at the end of prolonged treadmill exercise the vast majority of the runners complained about pain and soreness of their quadriceps rather than gastrocnemius muscles. In a pilot study on two subjects, very low glycogen concentrations in the vastus lateralis muscle $\left(36-38 \mathrm{mmol} . \mathrm{kg} \mathrm{dm}^{-1}\right)$ were observed at the end of exhaustive treadmill running. It was hypothesised therefore that the quadriceps muscle is heavily involved during treadmill running. 


\section{Treatment and storage of muscle samples}

All muscle samples were immersed in liquid nitrogen within 2-3 s of sampling. The samples were then quickly removed from the needle, placed in plastic screw-top tubes (eppendorf $1.2 \mathrm{ml}$ vol.), and kept in liquid nitogen for $12-24 \mathrm{~h}$ until they were freeze dried $(12 \mathrm{~h})$.

\footnotetext{
8 weef $12-15$
}

Each sample was then divided into two parts before being stored at $-70^{\circ} \mathrm{C}$ in two plastic tubes. At a later date, one part of the sample was used for single fibre analysis; the other part of the freeze-dried muscle was dissected free of visible blood and connective tissue, powdered using an agate pestle and mortar, and placed in plastic tubes. The powdered sample was then washed twice with $1 \mathrm{ml}$ petroleum ether $\left(30-400^{\circ} \mathrm{C}\right)$ to remove the fat. Each time the sample was mixed and centrifuged before removing the ether. Finally, the tube was left open in a fume cupboard until the sample was completely dry. The powdered samples were weighed using an elecrical balance accurate to five decimal places, placed in plastic tubes and stored at $-70^{\circ} \mathrm{C}$. At a later date, the powdered muscle samples were analysed for mixed muscle metabolites.

\section{Mixed muscle metabolite analysis}

Extraction procedure: On the day of the analysis, mixed muscle metabolites were extracted by adding $1 \mathrm{ml}$ of $0.5 \mathrm{mmol}^{-1}$ perchloric acid $\left(\mathrm{HClO}_{4}\right)$, containing 1 mmol.1-1 EDTA.Na2 (ethylenediamine tetra-acetic sodium salt), for every $10 \mathrm{mg}$ of powder kept in liquid nitrogen so that the extracting solution froze on introduction into the tube (Harris et al. 1974). Each sample was then placed in the freezer at $-20{ }^{\circ} \mathrm{C}$ for a few minutes for the extracting solution to thaw. The samples were then placed on ice and agitated using a Stuart flask shaker for $30 \mathrm{~min}$, and then centrifuged at $6000 \mathrm{rpm}$ for $10 \mathrm{~min}$ at 3-4 ${ }^{\circ} \mathrm{C}$. The supernatant was then removed using a pipette, carefully measured, placed into clean plastic tubes, and neutralised by adding onefourth volume of 2.1 mol. $\mathrm{l}^{-1}$ potassium bicarbonate $\left(\mathrm{KHCO}_{3}\right)$. The samples were then mixed and left in the fridge at $3-4{ }^{\circ} \mathrm{C}$ with loose caps to let the formed $\mathrm{CO}_{2}$ to escape, before centrifuged for $5 \mathrm{~min}$ at 3-4 ${ }^{\circ} \mathrm{C}$. Following this, the supernatant was removed, placed into clean plastic tubes and kept on ice. The $\mathrm{pH}$ of the extract was found to be 7.0 , and $1 \mathrm{ml}$ of it was equivalent to $8 \mathrm{mg}$ of muscle powder (Harris et al. 1974). 
Mixed muscle metabolite assays: All assays for each neutralised muscle extract were performed within two days. The analysis of ATP, $\mathrm{PCr}$ and $\mathrm{Cr}$ was performed on the first day immediately following the extraction procedure. The remaining extract was stored at $-70^{\circ} \mathrm{C}$. The free glucose, G-6-P, lactate and glycogen were assayed on the following day. The concentration of these metabolites has been shown to be unaffected by repeated freeze-thaw cycles of the muscle extracts. Glycogen was assayed by hydrolysis in 1 mol.1-1 hydrochloric acid $(\mathrm{HCl})$ both on the neutralised extract (acid-soluble glycogen) and on the precipitated muscle pellet after the extraction procedure (acidinsoluble glycogen). The total mixed muscle glycogen concentration was calculated by adding the acid-soluble and acid-insoluble glycogen concentrations.

The muscle extracts were assayed for the different metabolites using modifications of the methods described by Harris et al. (1974) and Lowry and Passonneau (1972). Acid-insoluble glycogen was assayed photometrically (Cecil, CE 393) for glucose using a commercially available glucose kit (Boeringher Mannheim-Glucose test combination, GOD/Perid method). The rest of the muscle metabolites were assayed flurometrically (Locarte, model 89). Their analysis was based on enzyme catalysed reactions, the coenzymes $\mathrm{NAD}^{+}$and $\mathrm{NADP}^{+}$being simultaneously reduced to NADH and NADPH, respectively. Duplicate analysis was performed on every sample. Detailed procedures of every assay performed are presented in Appendix A.

Buffers, co-factors (Grade-I) and enzymes were obtained as standard commercial items from Boehringer and Sigma. All reagents were made up with double-distilled water the day before the analysis and kept at $4{ }^{\circ} \mathrm{C}$. Standards were prepared on the day of the analysis using Grade-I chemicals. The molarity of the standards was checked on the same day spectrophotometrically using the coefficient of extinction of NADH (Appendix $B)$. The standard curves were always linear $(r=0.999$ to $r=1.000)$. Metabolite concentrations were calculated using the resulting standard regression equation, and taking into account double-distilled water blanks, extract blanks, standards, dilution factors and the extraction factor. Where necessary, the concentrations of the metabolites were adjusted to the true molarity of the standards as calculated from the coefficient of extinction of NADH, and to the highest content of total creatine $(\mathrm{PCr}+\mathrm{Cr})$ in the samples of each subject. The aim of the latter.adjustment was to compensate for any admixture of elements 
like connective tissue and fat droplets, and also blood contamination of the muscle sample.

The coefficient of variation for all the assays is shown in Table 3.3. All muscle metabolite concentrations were determined in dry weight to avoid changes in concentration due to water shift during exercise.

Table 3.3: Coefficient of variation of muscle metabolite assays $(n=15)$.

$\begin{array}{ll}\text { Acid insoluble glycogen } & 0.9 \% \\ \text { Acid soluble glycogen } & 3.0 \% \\ \text { Adenosine triphosphate } & 3.2 \% \\ \text { Phosphocreatine } & 1.8 \% \\ \text { Creatine } & 2.7 \% \\ \text { Glucose } & 3.5 \% \\ \text { Glucose-6-phosphate } & 3.4 \% \\ \text { Lactate } & 1.6 \%\end{array}$


Separation and identification of individual fibres: The second part of the freeze-dried muscle kept at $-70^{\circ} \mathrm{C}$ was used to determine the glycogen content of single muscle fibres. This part was also washed twice with petroleum ether to remove the fat, and then left in the fume cupboard to dry.

Fragments of single muscle fibres were dissected under low power microscopy (magnification $\times 10$ ). Three pieces from the end of each individual fibre fragment were then cut off, mounted on three different glass slides and stored at $-70^{\circ} \mathrm{C}$. The remaining part of the individual fibre was then weighed on a quartz-fibre fishpole balance (Lowry and Passonneau, 1972) before being placed in a methanol-washed plastic tube (eppendorf $1.2 \mathrm{ml}$ vol.) and stored at -70 ${ }^{\circ} \mathrm{C}$. The reproducibility of the weight of a single fibre was $1 \%$.

At a later date, the mounted ends of the fibre fragments were stained in duplicate, and where necessary in triplicate, for myofibrillar ATPase to identify Type I (slow-twitch) and Type II (fast-twitch) fibres using a modification of the method of Brooke and Kaiser (1970). Following the identification of each dissected fibre, 5-10 fibres of each type were pooled for each sample, the total weight ranging from 15 to $30 \mu \mathrm{g}$. The pooled fibres were placed at the bottom of methanol-washed plastic tubes and stored at $-70^{\circ} \mathrm{C}$ until analysis.

Extraction procedure: On the day of the analysis, the tubes with the pooled fibres were removed from the freezer, left to thaw and centrifuged to ensure all fibres were gathered at the bottom of the tube. Glycogen was then extracted by adding $20 \mu \mathrm{KOH}\left(1 \mathrm{mol.1} \mathrm{l}^{-1}\right)$ to each tube. Following this, samples were gently agitated on a vortex mixer and then warmed in a water bath at $50^{\circ} \mathrm{C}$ for $15 \mathrm{~min}$. Samples were then removed from the water bath, and $60 \mu \mathrm{l}$ of doubledistilled de-ionised water was added before being mixed. This procedure resulted in the complete digestion of the fibres. The volume of each muscle extract was $80 \mu \mathrm{l}$.

Glycogen degradation: Following the extraction procedure, the extract of each muscle sample was neutralized by adding $80 \mu \mathrm{l}$ of $\mathrm{HCl} /$ Acetate buffer $(0.25$ mol..$^{-1} \mathrm{HCl}$ and $0.15 \mathrm{~mol}^{-1} \mathrm{l}^{-1}$ Acetate) to two tubes, each containing $40 \mu \mathrm{l}$. For every series of samples being degraded, one control sample (containing $40 \mu \mathrm{l}$ of $0.25 \mathrm{~mol} . \mathrm{l}^{-1} \mathrm{KOH}$ and $80 \mu \mathrm{l}$ of $\mathrm{HCl} /$ Acetate buffer) was also used. All samples 
were then mixed, and $4 \mu \mathrm{l}$ of the enzyme amyloglucosidase ( 6 units.mg ${ }^{-1}$ ) was added to the control sample $(\mathrm{Zc})$ and to one tube of each pair of muscle extracts $(\mathrm{Za})$, before being mixed once again. The other tube with the neutralised extract was analysed without prior glycogen degradation $(\mathrm{Zb})$. Samples were left to stand for $60 \mathrm{~min}$ at room temperature, centrifuged for $2 \mathrm{~min}$ and the supernatant was then removed, placed into clean plastic tubes and put on ice.

Glycogen assay: The muscle extract was assayed fluorometrically for glucose using a modification of the method by Harris et al. (1974). Detailed procedures of this assay are presented in Appendix $C$. The coefficient of variation of the assay was $4.5 \%$. Glucose concentration in each cuvette was calculated using a NADH standard curve, since the emmision light from the fluorimeter lamp was not stable. The slope of the NADH curve enabled the values determined by the fluorimeter to be related to the values accurately determined spectrophotometrically (see Appendix C). The glycogen concentration of each sample was calculated using the glucose concentration in cuvette, and taking into account double-distilled water blanks, control samples $(\mathrm{Zc})$, dilution and extraction factors and the total weight of the pooled fibres (see Appendix $C$ ).

In studies 3 and 4 , single fibre analysis was performed on the biopsy samples of six subjects. As with the mixed muscle metabolites, single muscle fibre glycogen concentration was expressed as mmol.kg dm-1. 


\section{CHAPTER 4}

\section{INFLUENCE OF CARBOHYDRATE-ELECTROLYTE DRINKS ON MARATHON RUNNING PERFORMANCE}

\subsection{INTRODUCTION}

Fatigue during prolonged heavy exercise is associated with glycogen depletion in working muscles (Hermansen et al. 1967). A reduction in liver glycogen stores and subsequent decrease in blood glucose concentration has also been identified as a possible cause of fatigue (Christensen and Hansen, 1939; Coyle et al. 1983). Therefore, it is not surprising that methods of increasing muscle glycogen concentrations before prolonged exercise have been successfully developed (Bergstrom et al. 1967; Sherman et al. 1981). Effective ways of supplementing the body's limited carbohydrate stores during exercise have also been the focus of attention over the last decade. Drinking carbohydrate-electrolyte beverages during exercise has the advantage that they not only provide a way of supplementing the body's limited carbohydrate stores but they also help off-set fluid lost from sweating and so delay the onset of severe dehydration (Coyle and Montain, 1992b).

The performance benefits of drinking carbohydrate-electrolyte solutions during prolonged submaximal cycling have been clearly confirmed in several studies (Coyle et al. 1983; 1986; Maughan et al. 1989; Wright et al. 1991). A wide range of concentrations of carbohydrate solutions have been shown to be effective in delaying fatigue and increasing endurance capacity (Lamb and Brodowicz, 1986).

Relatively few studies, however, have examined the influences of drinking carbohydrate-electrolyte solutions on running performance. In one of the few studies on the effects of drinking a dilute carbohydrateelectrolyte beverage on running performance, a higher running speed was observed at the end of a $30-\mathrm{km}$ treadmill time trial than when only water was ingested throughout the simulated race (Williams et al. 1990). However, there was no significant difference between the overall performance times during the two trials. In a subsequent study we found, 
using the same carbohydrate-electrolyte solution and experimental design, that the performance times in a $30-\mathrm{km}$ road race were significantly faster when the runners consumed the carbohydrate solution than when they drank water (Tsintzas et al. 1993). Only a limited number of measurements were made in these two studies and so we were unable to offer any metabolic or thermoregulatory explanations for the difference in running speeds between the trials. Furthermore, the differences in running speeds appeared only towards the end of the $30-\mathrm{km}$ races and so it is not unreasonable to suggest that simulated races over longer distances would show the effects of drinking a carbohydrate-electrolyte solution more clearly.

The purpose, therefore, of the present study was to examine the effects on performance of drinking two carbohydrate-electrolyte solutions and water during three treadmill marathons $(42.2 \mathrm{~km})$. 


\subsection{METHODS}

Subjects: Seven endurance-trained male runners volunteered to take part in this study. The subjects' mean ( \pm SEM) age, height, weight, $\mathrm{VO}_{2} \max$ and heart rate max were $44 \pm 5 \mathrm{yrs}, 176 \pm 2 \mathrm{~cm}, 66.0 \pm 2.4 \mathrm{~kg}, 58.4 \pm 1.4 \mathrm{ml} . \mathrm{kg}^{-}$ $1 . \mathrm{min}^{-1}$ and $180 \pm 5 \mathrm{bt} \cdot \mathrm{min}^{-1}$, respectively. The subjects covered between 65 and $160.9 \mathrm{~km}$ (40 to 100 miles) a week in training. Most of them had completed several marathon and ultramarathon races. As an additional measure of the fitness of each runner the $\% \mathrm{VO}_{2}$ max and running speed at blood lactate concentration of 2 mmol..$^{-1}$ were determined and found to be $78.7 \pm 6.8 \%$ and $3.99 \pm 0.48 \mathrm{~m} . \mathrm{s}^{-1}$, respectively.

Preliminary measurements: A series of preliminary tests were conducted after the subjects became familiar with the experimental procedures and treadmill running (see Chapter 3) in order to determine: (i) the relationship between running speed and oxygen uptake, (ii) the maximum oxygen uptake ( $\mathrm{VO}_{2}$ max), and (iii) the running speed and percentage $\mathrm{VO}_{2}$ max equivalent to a blood lactate concentration of $2 \mathrm{mmol.1^{-1 }}$ and 4 mmol.1-1. A prolonged treadmill run $(60-90 \mathrm{~min})$ was also performed by each subject prior to the first treadmill marathon. A difference in performance time was observed between runs 1 and 2, but not between runs 2 and 3 (run 1: $196.6 \pm 6.0 \mathrm{~min}$, run 2: $190.6 \pm 11.3 \mathrm{~min}$, run 3: $189.0 \pm$ 10.6). However, this difference between runs 1 and 2 could be due to the fact that during run 1 three subjects ingested water, during run 2 three ingested the A solution and during run 3 three ingested the B solution.

Protocol of the study: The runners were required to complete the marathon distance $(42,195 \mathrm{~m})$ in the fastest possible time on three occasions, 4 weeks apart (see Chapter 3). All three trials were performed in the laboratory on a level motorised treadmill under similar experimental and environmental conditions. On each occasion the subjects had to ingest either tap water ( $W$ trial), a 5.5\% lemon-lime flavoured carbohydrate solution (A trial) or a $6.9 \%$ orange-flavoured carbohydrate solution (B trial) in a series of feedings every $5 \mathrm{~km}$ of the run (Fig. 4.1). All trials were conducted following a 2-day normal standardised diet and a 10-12 hour overnight fast. Just before the warm-up and while standing on the treadmill the runners consumed $3 \mathrm{ml} . \mathrm{kg}^{-1}$ body weight (bw) of the assigned fluid. A further $2 \mathrm{ml} \cdot \mathrm{kg}^{-1}$ bw of the assigned fluid was ingested by the 
subjects every $5 \mathrm{~km}$ of the run. The warm-up consisted of 5 -min run at $60 \% \mathrm{VO}_{2}$ max. Immediately after the completion of the warm-up the running speed increased to $70 \% \mathrm{VO}_{2}$ max. In order to compare the effects of the three fluids on metabolism during running at a constant speed, the subjects ran the first $5 \mathrm{~km}$ of each trial at speed equivalent to $70 \% \mathrm{VO}_{2}$ max. Thereafter, they selected their own speed in order to cover the full marathon distance in the fastest possible time.

Venous blood samples were obtained at rest, at the end of the warm-up, every $5 \mathrm{~km}$ of the run and at the end of the marathon. One minute expired air samples were collected during the last minute of the warm-up, every 5 $\mathrm{km}$ of the run and during the final minute of the run. A rating of perceived exertion (Borg, 1973) and a simple 1-10 scale of perceived thirst were used at the same intervals. Following expired air and blood sampling, the assigned fluid was ingested by the subjects every $5 \mathrm{~km}$. The last fluid ingestion took place at $35 \mathrm{~km}$ of the run. Wet sponges were available to the subjects for use ad libitum throughout the runs. Heart rate and skin and rectal temperatures were continuously monitored and recorded throughout the marathons. The subject's body weight in nude was also obtained before and after the marathon run. The subjects returned to the laboratory approximately 24 hours after the completion of each $42.2-\mathrm{km}$ run where a venous blood sample was obtained for the determination of plasma creatine kinase concentration.

Analysis: Expired air samples were collected and analysed as previously described (see Chapter 3). Venous blood samples were analysed for blood lactate, glucose and haemoglobin concentrations, and haematocrit values; plasma ammonia, sodium, potassium, FFA, glycerol, urea, creatine kinase, insulin, cortisol, growth hormone, adrenaline and nor-adrenaline concentrations. Changes in plasma volume were measured as previously described (see Chapter 3).

Statistics: A two-way or one-way analysis of variance (ANOVA) were used where appropriate to compare differences between the three trials. Any significant differences shown were assessed by applying the Tukey post-hoc test. Statistical significance was accepted at $5 \%$ level. Results are presented as mean $\pm \mathrm{SEM}$. 
Fluid ingestions

Heart rate and body temperature measurements
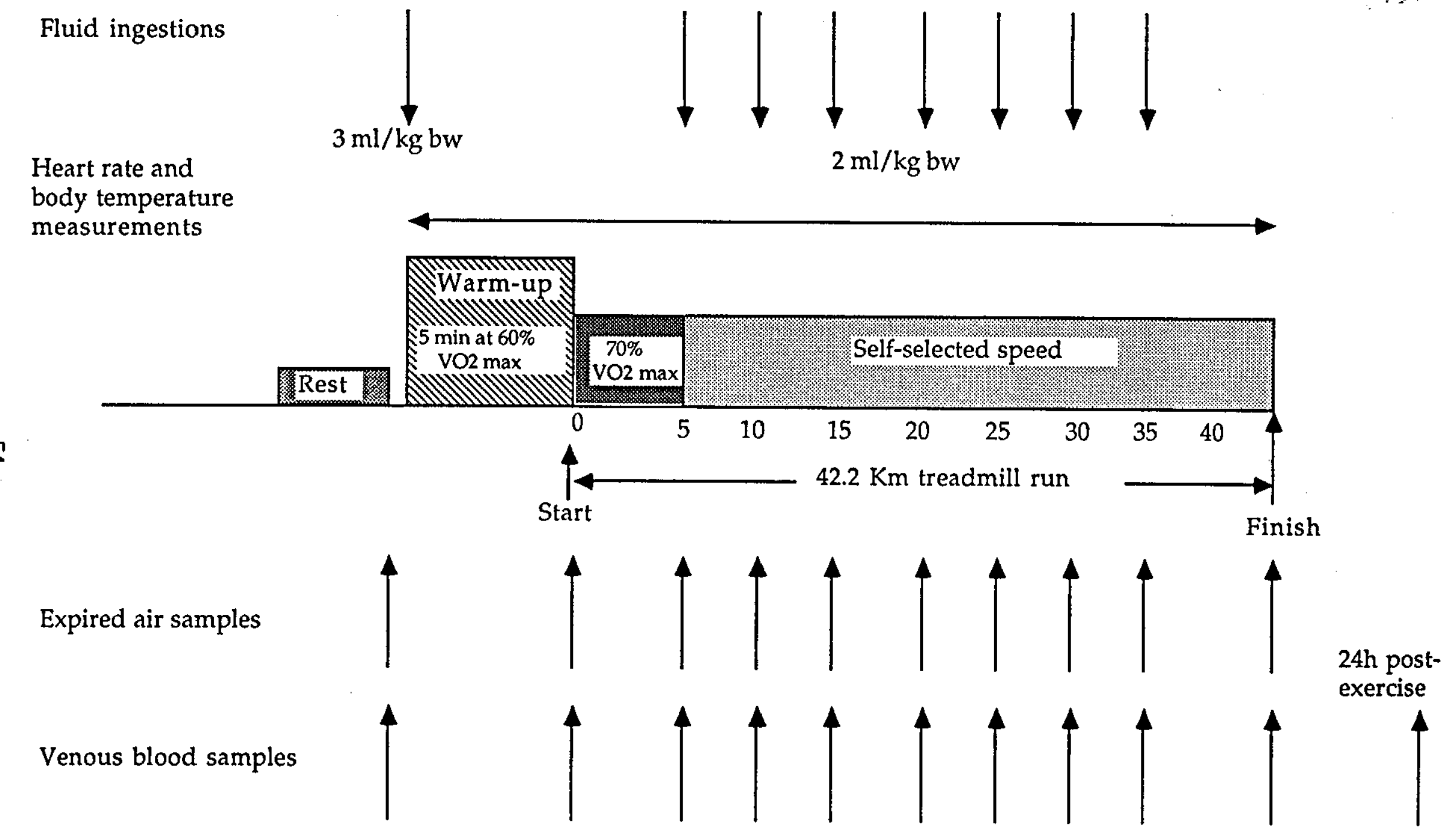


\subsection{RESULTS}

All three trials were conducted under similar environmental conditions. Dry bulb temperatures were $20.3 \pm 0.2{ }^{\circ} \mathrm{C}, 20.5 \pm 0.2{ }^{\circ} \mathrm{C}$ and $20.2 \pm 0.3^{\circ} \mathrm{C}$ during the $\mathrm{W}, \mathrm{B}$ and $\mathrm{A}$ trials respectively and relative humidity for the three conditions were $49.0 \pm 2.4 \%, 48.0 \pm 2.1 \%$ and $52.8 \pm 2.4 \%$ respectively.

Only during the A trial were the runners able to maintain their chosen speeds throughout the entire marathon run. Running speeds at the end of the $\mathrm{W}$ and $B$ trials were slower $(\mathrm{p}<0.05)$ than the speeds at $10 \mathrm{~km}$ and 25 $\mathrm{km}$ respectively (Fig. 4.2). As a result, overall performance time was faster $(p<0.05)$ for the A trial compared with the $W$ trial (Table 4.1). No differences were observed between either the $W$ and $B$ trials or the $B$ and A trials. Performance times, up to $25 \mathrm{~km}$ of the race, were similar in all three trials. It was over the last $17.2 \mathrm{~km}$ during the A trial that the subjects ran faster $(\mathrm{p}<0.01)$.

The mean $\% \mathrm{~V}_{2}$ max sustained during the $\mathrm{W}, \mathrm{B}$ and $\mathrm{A}$ runs were similar between trials $(70.1 \pm 2.1 \%$ vs $70.1 \pm 2.2 \%$ vs $73.5 \pm 3.1 \%$ respectively). However, at the end of the A trial the relative exercise intensity (\% $\mathrm{VO}_{2}$ max) was higher $(p<0.01)$ than that at the end of the $W$ trial. There was no difference between $W$ and $B$ trials (Table 4.2).

The heart rates during the marathon runs were similar in all three trials (Table 4.2). The average heart rates were $154 \pm 2,159 \pm 3$ and $157 \pm 2$ bt . in $^{-}$ 1 for the $W, B$ and $A$ trials respectively. The average heart rate during the $B$ trial was higher than the values observed during the $W$ trial $(p<0.05)$.

The average daily energy intake of the runners and the percentage contribution from carbohydrate for the two days preceding each marathon were $13.6 \pm 0.6 \mathrm{MJ}$ and $48.2 \pm 6.8 \%$ respectively. Running the treadmill marathon resulted in average energy expenditures of $10.0 \mathrm{MJ}$ during the $\mathrm{W}, \mathrm{B}$ and $\mathrm{A}$ trials.

Total volumes of fluid ingested throughout the $\mathrm{W}, \mathrm{B}$ and $\mathrm{A}$ trials were $1112 \pm 42 \mathrm{ml}, 1116 \pm 44 \mathrm{ml}$ and $1100 \pm 44 \mathrm{ml}$ respectively. During the $B$ trial the subjects ingested a total of $77 \pm 8 \mathrm{~g}$ of carbohydrate at a rate of $24 \pm 2$ g.h ${ }^{-1}$. In contrast, owing to the slightly lower carbohydrate concentration 
of the A drink, only $60 \pm 7 \mathrm{~g}$ carbohydrate was ingested during this trial, resulting in a carbohydrate provision rate of $19 \mathrm{~g} . \mathrm{h}^{-1}$.

There were no differences in the respiratory exchange ratios (RER values) between the three trials at any time during the marathons (Table 4.2). At the end of the marathon RER values were $0.77 \pm 0.02,0.82 \pm 0.02$ and $0.81 \pm$ 0.02 in the $W, B$ and $A$ trials respectively. The overall average rate of carbohydrate oxidation, as calculated from $\mathrm{VO}_{2}$ and $\mathrm{RER}$ values, was also similar in the $W, B$ and A trials $\left(1.2 \pm 0.1 \mathrm{~g} \cdot \mathrm{min}^{-1}\right.$ vs $1.5 \pm 0.1 \mathrm{~g} \cdot \mathrm{min}^{-1}$ vs 1.5 \pm 0.2 g. $\min ^{-1}$ ).

No differences were found between trials for perceived rate of exertion and perceived rate of thirst. Changes in body weight $(3.6 \pm 0.2 \mathrm{~kg}$ vs $3.7 \pm 0.2 \mathrm{~kg}$ vs $3.7 \pm 0.1 \mathrm{~kg})$, plasma volume $(-0.7 \pm 2.8 \%$ vs $+1.3 \pm 3.1 \%$ vs $+3.3 \pm 2.8 \%)$, average sweat rate $\left(1.0 \pm 0.11 . \mathrm{h}^{-1}\right.$ vs $1.0 \pm 0.11 . \mathrm{h}^{-1}$ vs $\left.1.0 \pm 0.1 \mathrm{l} . \mathrm{h}^{-1}\right)$ and rectal temperature were also not different between the $\mathrm{W}, \mathrm{B}$ and $\mathrm{A}$ trials respectively. Rectal temperature values at the end of the $W, B$ and $A$ trials were $38.4 \pm 1.0^{\circ} \mathrm{C}, 38.4 \pm 0.8{ }^{\circ} \mathrm{C}$ and $38.9 \pm 0.2^{\circ} \mathrm{C}$ respectively. In contrast, mean skin temperature was higher $(\mathrm{p}<0.05)$ in the A trial compared with both the $\mathrm{W}$ and $\mathrm{B}$ trials $\left(30.9 \pm 0.1{ }^{\circ} \mathrm{C}\right.$ vs $29.4 \pm 0.3^{\circ} \mathrm{C}$ vs $29.8 \pm 0.2{ }^{\circ} \mathrm{C}$ respectively).

Carbohydrate supplementation during the $42.2 \mathrm{~km}$ treadmill run did not result in higher blood glucose concentrations than during the $W$ trial (Fig. 4.3). However, during the $W$ trial blood glucose concentration at $15 \mathrm{~km}$ rose $(\mathrm{p}<0.05)$ above post-warm up values. Blood glucose was higher $(\mathrm{p}<$ $0.01)$ at the end of the $B$ trial than pre-exercise values.

There was no difference between the three trials in blood lactate concentrations (Fig. 4.4) and in plasma sodium and potassium concentrations. Post-exercise plasma sodium and potassium concentrations were not different from resting values in all three trials (Sodium: $136 \mathrm{mmol} .1^{-1}$; Potassium: $3.9 \mathrm{mmol}^{-1}{ }^{-1}$ )

Plasma ammonia concentration increased during all three trials. At the end of the A trial it was higher $(p<0.01)$ than the value obtained at the end of the $W$ trial (Fig. 4.5). There were strong correlations between performance times for $\mathrm{W}$ and $\mathrm{A}$ trials and plasma ammonia 
concentrations at $42.2 \mathrm{~km}(\mathrm{r}=-0.847, \mathrm{p}<0.05$ and $\mathrm{r}=-0.920, \mathrm{p}<0.01$ respectively). Plasma urea concentrations were also higher $(p<0.01)$ than pre-exercise values at the end of the $W, B$ and $A$ trials $\left(7.5 \pm 0.5\right.$ mmol. $1^{-1}$ from $6.1 \pm 0.5 \mathrm{mmol}^{-1}$ vs $7.4 \pm 0.4 \mathrm{mmol}^{-1}$ from $6.2 \pm 0.4 \mathrm{mmol}^{-1} \mathrm{1}^{-1} \mathrm{vs}$ $7.7 \pm 0.3 \mathrm{mmol} .1^{-1}$ from $6.4 \pm 0.4 \mathrm{mmol} . \mathrm{1}^{-1}$ respectively). No significant difference was observed, however, between the three trials.

An increase ( $p<0.01)$ in plasma FFA (Fig. 4.6) and plasma glycerol (Fig. 4.7) occurred at the end of the $42.2 \mathrm{~km}$ in all three trials. However, carbohydrate supplementation resulted in a lower $(p<0.05)$ plasma FFA and glycerol concentrations at $42.2 \mathrm{~km}$ when compared with the water trial

The hormonal responses to fluid ingestion during the $42.2 \mathrm{~km}$ run are shown in Table 4.3. When compared with resting values, plasma insulin concentration at the end of the run was lower $(p<0.05)$ in the $W$ and $A$ trials. Plasma cortisol concentration at $42.2 \mathrm{~km}$ was higher than preexercise values in all three trials (Table 4.3). Lower resting plasma growth hormone $(\mathrm{GH})$ concentration $(p<0.05)$ was observed in the $B$ trial than in the $\mathrm{W}$ trial. During exercise plasma $\mathrm{GH}$ rose only in the $\mathrm{B}$ trial $(\mathrm{p}<0.05)$. Plasma adrenaline concentrations at $42.2 \mathrm{~km}$ were higher than resting values in the $W$ and $A$ trials $(p<0.05)$. Elevated plasma nor-adrenaline concentrations were observed at the end of all three trials $(p<0.05)$. There was no difference between trials for plasma insulin, cortisol, $G H$, adrenaline and nor-adrenaline at the end of the marathon runs.

Plasma creatine kinase concentrations rose during the $42.2 \mathrm{~km}$ run in all trials (Fig. 4.8). No difference was observed between trials. During the recovery period plasma creatine kinase continued to rise and the day after the marathon it was higher $(p<0.05)$ than the values obtained immediately after each run (Fig. 4.8). Furthermore, plasma creatine kinase was higher $(p<0.05)$ in the A trial than in the $W$ trial. 
Table 4.1 : Performance times (min) for $W, B$ and $A$ trials during the $42.2-\mathrm{km}$ run (mean \pm $\mathrm{SEM}, \mathrm{n}=7$ )

\begin{tabular}{|c|c|c|c|c|c|c|c|c|}
\hline \multirow[t]{2}{*}{ Trial } & \multicolumn{8}{|c|}{ Distance (km) } \\
\hline & 5 & 10 & 15 & 20 & 25 & 30 & 35 & 42.2 \\
\hline \multirow[t]{2}{*}{$\mathbf{w}$} & $\overline{23.6}$ & 45.5 & 67.4 & 89.5 & 112.0 & 135.2 & 158.8 & 193.9 \\
\hline & \pm 0.9 & \pm 1.3 & \pm 1.8 & \pm 2.1 & \pm 2.5 & \pm 3.2 & \pm 3.9 & \pm 5.0 \\
\hline \multirow[t]{2}{*}{ B } & 23.7 & 46.2 & 68.4 & 90.4 & 112.0 & 134.2 & 156.9 & 192.4 \\
\hline & \pm 0.9 & \pm 1.3 & \pm 1.7 & \pm 1.9 & \pm 2.2 & \pm 2.5 & \pm 2.9 & \pm 3.3 \\
\hline \multirow[t]{2}{*}{$\mathbf{A}$} & 23.6 & 46.1 & 68.1 & 89.7 & 111.9 & 134.0 & 156.8 & $190.0^{*}$ \\
\hline & \pm 0.9 & \pm 1.3 & \pm 1.7 & \pm 2.1 & \pm 2.4 & \pm 2.7 & \pm 3.1 & \pm 3.9 \\
\hline
\end{tabular}


Table 4.2: VO2 (1.min-1), \%VO2 max, Heart Rate (HR) (bts.min ${ }^{-1}$ ) and Respiratory Exchange Ratio (RER) in the W, B and A trials during the 42.2-km run ( $\mathrm{n=7}$ )

\begin{tabular}{|c|c|c|c|c|c|c|}
\hline $\begin{array}{c}\text { Distance } \\
(\mathbf{k m})\end{array}$ & Trial & & VO2 & $\begin{array}{l}\text { \%VO2 } \\
\max \end{array}$ & HR & RER \\
\hline 5 & $\begin{array}{l}\text { W } \\
\text { B } \\
\text { A }\end{array}$ & $\begin{array}{l}\text { mean } \\
\pm \mathrm{SEM} \\
\text { mean } \\
\pm \mathrm{SEM} \\
\text { mean } \\
\pm \mathrm{SEM}\end{array}$ & $\begin{array}{l}2.5 \\
0.1 \\
2.5 \\
0.1 \\
2.6 \\
0.1\end{array}$ & $\begin{array}{r}66.3 \\
1.4 \\
65.4 \\
1.0 \\
66.6 \\
0.8\end{array}$ & $\begin{array}{r}139 \\
8 \\
141 \\
7 \\
144 \\
6\end{array}$ & $\begin{array}{l}0.83 \\
0.01 \\
0.84 \\
0.01 \\
0.85 \\
0.02\end{array}$ \\
\hline 15 & $\begin{array}{l}\text { W } \\
\text { B } \\
\text { A }\end{array}$ & $\begin{array}{l}\text { mean } \\
\pm S E M \\
\text { mean } \\
\pm S E M \\
\text { mean } \\
\pm S E M\end{array}$ & $\begin{array}{l}2.8 \\
0.1 \\
2.7 \\
0.1 \\
2.8 \\
0.1\end{array}$ & $\begin{array}{r}73.3 \\
2.0 \\
70.3 \\
2.4 \\
74.1 \\
3.3\end{array}$ & $\begin{array}{r}156 \\
6 \\
157 \\
6 \\
154 \\
4\end{array}$ & $\begin{array}{l}0.82 \\
0.01 \\
0.87 \\
0.02 \\
0.84 \\
0.02\end{array}$ \\
\hline 25 & $\begin{array}{l}\text { W } \\
\text { B } \\
\text { A }\end{array}$ & $\begin{array}{l}\text { mean } \\
\pm S E M \\
\text { mean } \\
\pm S E M \\
\text { mean } \\
\pm S E M\end{array}$ & $\begin{array}{l}2.6 \\
0.1 \\
2.7 \\
0.1 \\
2.8 \\
0.1\end{array}$ & $\begin{array}{r}69.3 \\
2.8 \\
72.3 \\
3.2 \\
74.5 \\
3.8\end{array}$ & $\begin{array}{r}157 \\
4 \\
163 \\
6 \\
160 \\
4\end{array}$ & $\begin{array}{l}0.81 \\
0.02 \\
0.86 \\
0.01 \\
0.83 \\
0.02\end{array}$ \\
\hline 35 & $\begin{array}{l}\text { W } \\
\text { B } \\
\text { A }\end{array}$ & $\begin{array}{l}\text { mean } \\
\pm S E M \\
\text { mean } \\
\pm S E M \\
\text { mean } \\
\pm S E M\end{array}$ & $\begin{array}{l}2.6 \\
0.1 \\
2.7 \\
0.1 \\
2.8 \\
0.2\end{array}$ & $\begin{array}{r}69.5 \\
3.2 \\
69.4 \\
3.7 \\
73.5 \\
5.1\end{array}$ & $\begin{array}{r}156 \\
5 \\
165 \\
5 \\
162 \\
4\end{array}$ & $\begin{array}{l}0.80 \\
0.01 \\
0.82 \\
0.01 \\
0.83 \\
0.02\end{array}$ \\
\hline 42.2 & $\begin{array}{l}\text { W } \\
\text { B } \\
\text { A }\end{array}$ & $\begin{array}{l}\text { mean } \\
\pm S E M \\
\text { mean } \\
\pm S E M \\
\text { mean } \\
\pm S E M\end{array}$ & $\begin{array}{l}2.5 \\
0.1 \\
2.7 \\
0.3 \\
2.9^{*} \\
0.2\end{array}$ & $\begin{array}{r}65.5 \\
4.8 \\
69.2 \\
6.6 \\
75.2^{*} \\
4.1\end{array}$ & $\begin{array}{r}159 \\
6 \\
163 \\
4 \\
164 \\
4\end{array}$ & $\begin{array}{l}0.77 a \\
0.02 \\
0.82 \\
0.02 \\
0.81 \\
0.02\end{array}$ \\
\hline
\end{tabular}

${ }^{*} \mathrm{p}<0.01$ from $W$ a $p<0.05$ from $5 \mathrm{~km}$ 


\begin{tabular}{|c|c|c|c|}
\hline $\begin{array}{l}\text { Plasma in } \\
\text { noradren } \\
\text { and after }\end{array}$ & $\begin{array}{l}\text { cortiso } \\
\text { concent } \\
.2-\mathrm{km} \mathrm{r}\end{array}$ & $\begin{array}{l}n \text { horm } \\
n \text { the } W \\
n \pm S E N\end{array}$ & $\begin{array}{l}\text { ne, adrenaline and } \\
B \text { and A trials before } \\
n=7)\end{array}$ \\
\hline Plasma hormone & Trial & Rest & $42.2 \mathrm{~km}$ \\
\hline Insulin (mU/1) & $\begin{array}{l}\text { W } \\
\text { B } \\
\text { A }\end{array}$ & $\begin{array}{l}5.7 \\
\pm 0.7 \\
6.3 \\
\pm 0.6 \\
6.1 \\
\pm 1.2\end{array}$ & $\begin{array}{l}3.1 \mathrm{~b} \\
\pm 0.4 \\
4.2 \\
\pm 0.5 \\
3.4 \mathrm{~b} \\
\pm 0.3\end{array}$ \\
\hline Cortisol (nmol/1) & $\begin{array}{l}\text { W } \\
\text { B } \\
\text { A }\end{array}$ & $\begin{array}{l}475 \\
\pm 31 \\
498 \\
\pm 41 \\
555 \\
\pm 39\end{array}$ & $\begin{array}{c}937 \mathrm{a} \\
\pm 117 \\
976 \mathrm{a} \\
\pm 101 \\
838 \mathrm{~b} \\
\pm 64\end{array}$ \\
\hline Growth hormone (mU/l) & $\begin{array}{l}\text { W } \\
\text { B } \\
\text { A }\end{array}$ & $\begin{array}{l}16.6 \\
\pm 4.7 \\
4.9 \mathrm{c} \\
\pm 1.8 \\
10.0 \\
\pm 3.7\end{array}$ & $\begin{array}{l}30.4 \\
\pm 7.2 \\
29.7 \mathrm{~b} \\
\pm 7.0 \\
23.5 \\
\pm 6.9\end{array}$ \\
\hline Adrenaline (nmol/l) & $\begin{array}{l}\text { W } \\
\text { B } \\
\text { A }\end{array}$ & $\begin{array}{l}0.56 \\
\pm 0.20 \\
0.63 \\
\pm 0.13 \\
0.40 \\
\pm 0.10\end{array}$ & $\begin{array}{l}1.29 \mathrm{~b} \\
\pm 0.29 \\
2.01 \\
\pm 0.92 \\
1.65 \mathrm{a} \\
\pm 0.36\end{array}$ \\
\hline Noradrenaline (nmol/l) & $\begin{array}{l}\text { W } \\
\text { B } \\
\text { A }\end{array}$ & $\begin{array}{l}2.16 \\
\pm 0.46 \\
2.05 \\
\pm 0.28 \\
1.98 \\
\pm 0.39\end{array}$ & $\begin{array}{l}8.39 \mathrm{~b} \\
\pm 1.74 \\
10.72 \mathrm{~b} \\
\pm 3.11 \\
9.34^{*} \\
\pm 1.55\end{array}$ \\
\hline
\end{tabular}

* $p=0.05$ from rest, a $p<0.01$ from rest, $b p<0.05$ from rest,

c $p<0.05$ from $W$ 


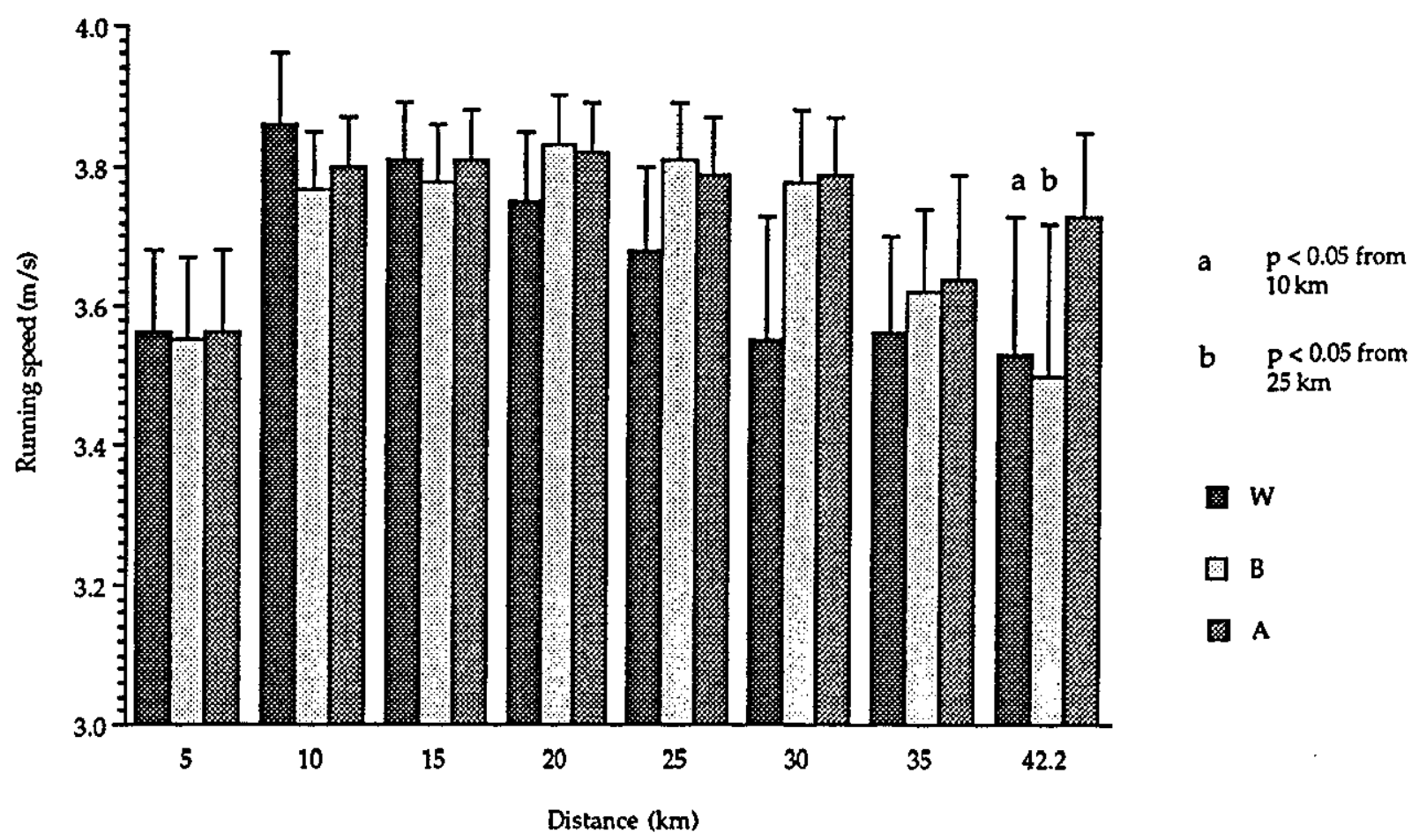

Fig. 4.2: Running speeds $(\mathrm{m} / \mathrm{s}$ ) for $W, B$ and $A$ trials during the $42.2-\mathrm{km}$ run (mean $\pm S E M, n=7$ ) 


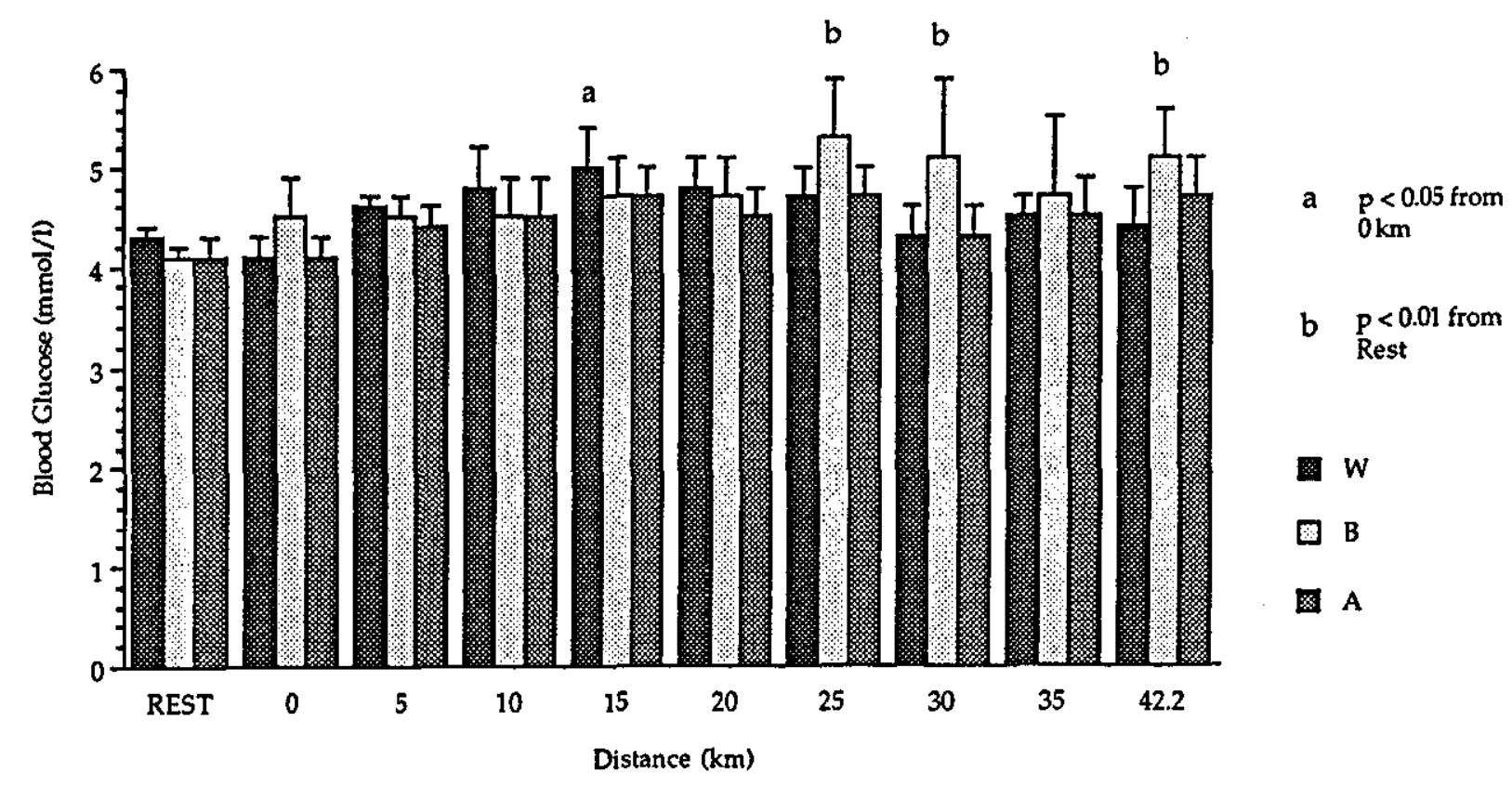

Fig. 4.3 : Blood glucose concentrations (mmol/l) during W, B and A trials over the $42.2-\mathrm{km}$ run (mean $\pm S E M, n=7$ )

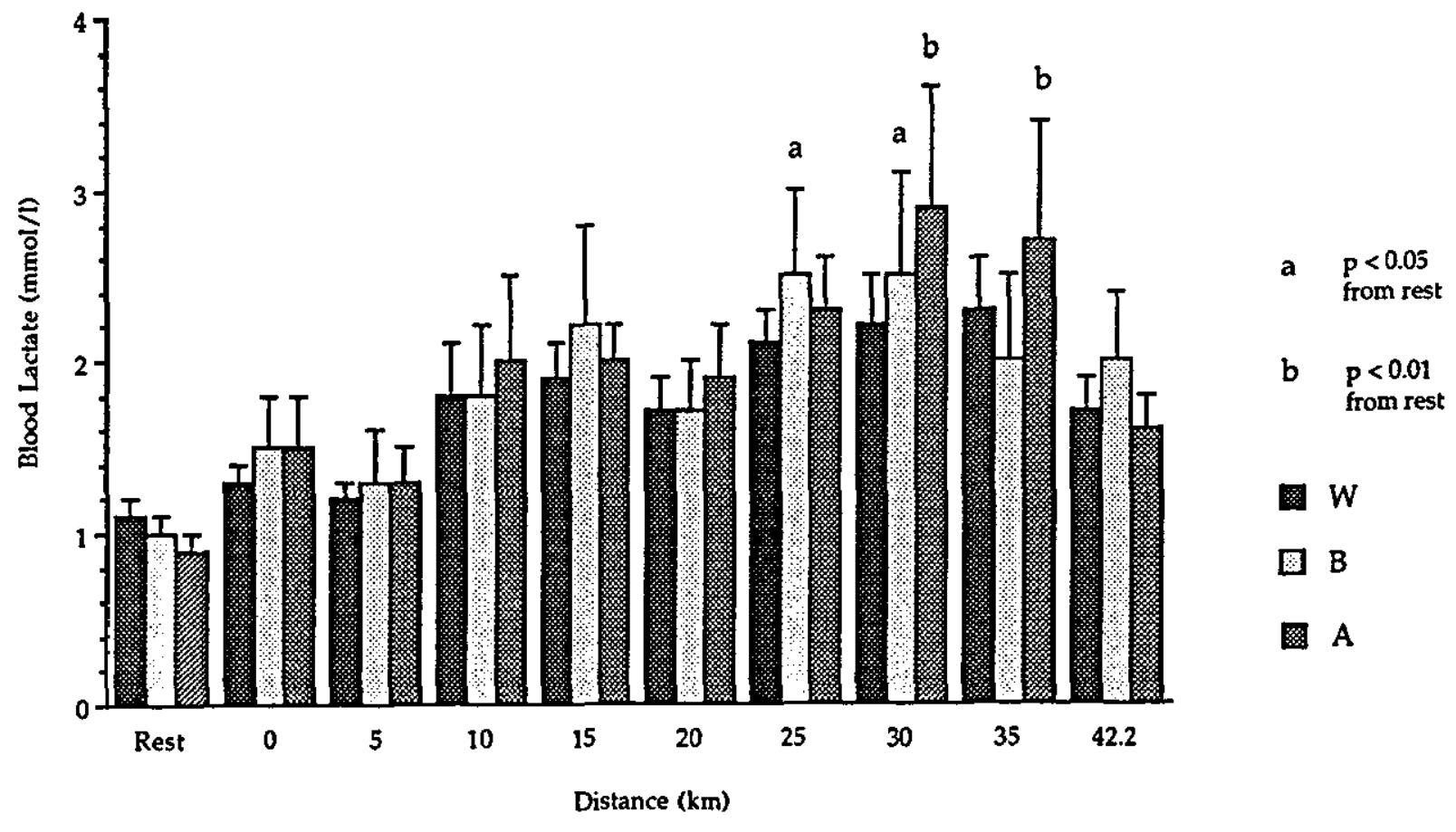

Fig. 4.4 : Blood lactate concentrations (mmol/l) during W, B and A trials over the $42.2 \mathrm{~km}$ run (mean $\pm S E M, n=7$ ) 


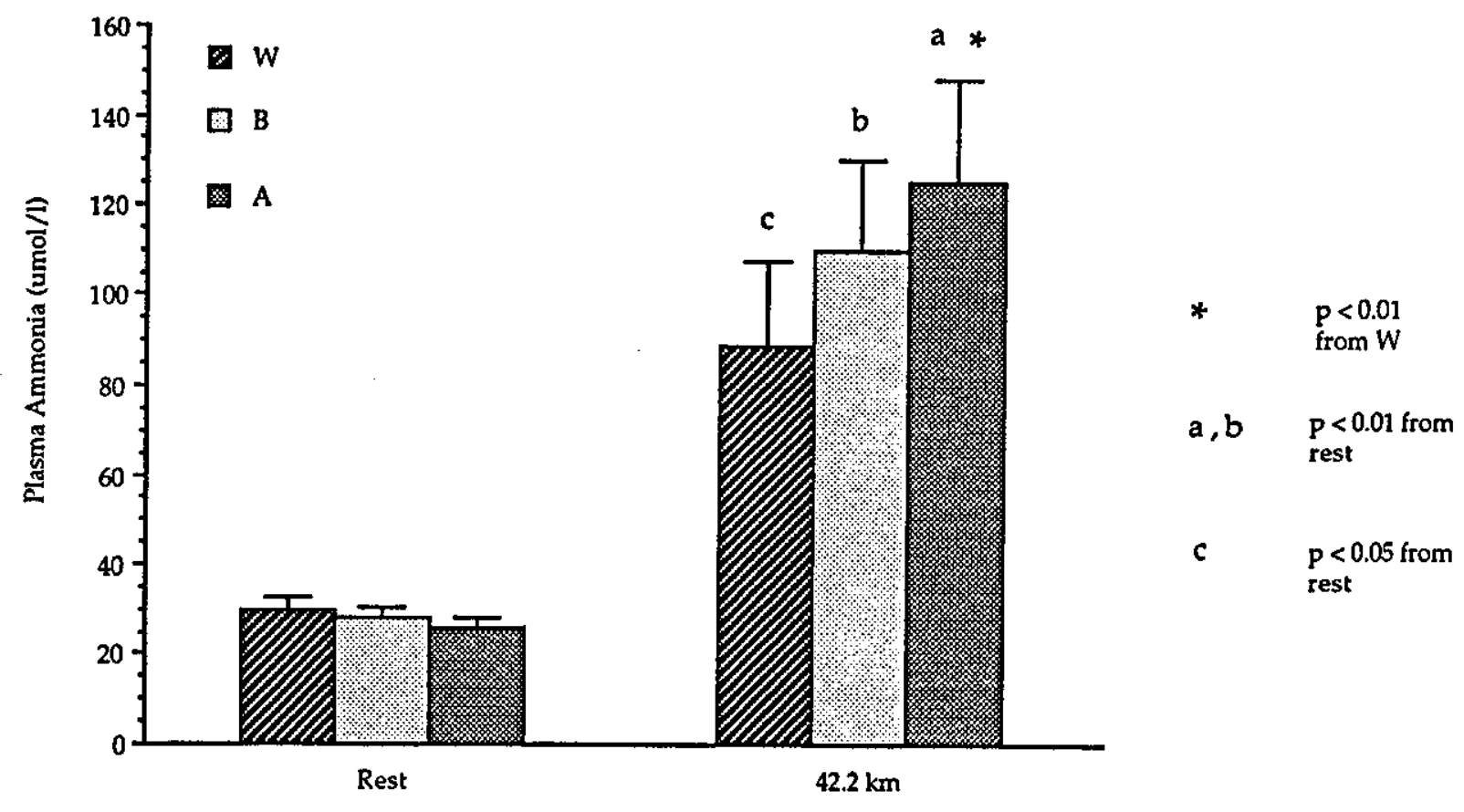

Fig. 4.5: Plasma ammonia concentrations ( $\mu$ mol/ $/$ ) for $W, B$ and $A$ trials during the 42.2-km run (mean $\pm S E M, n=7$ ) 


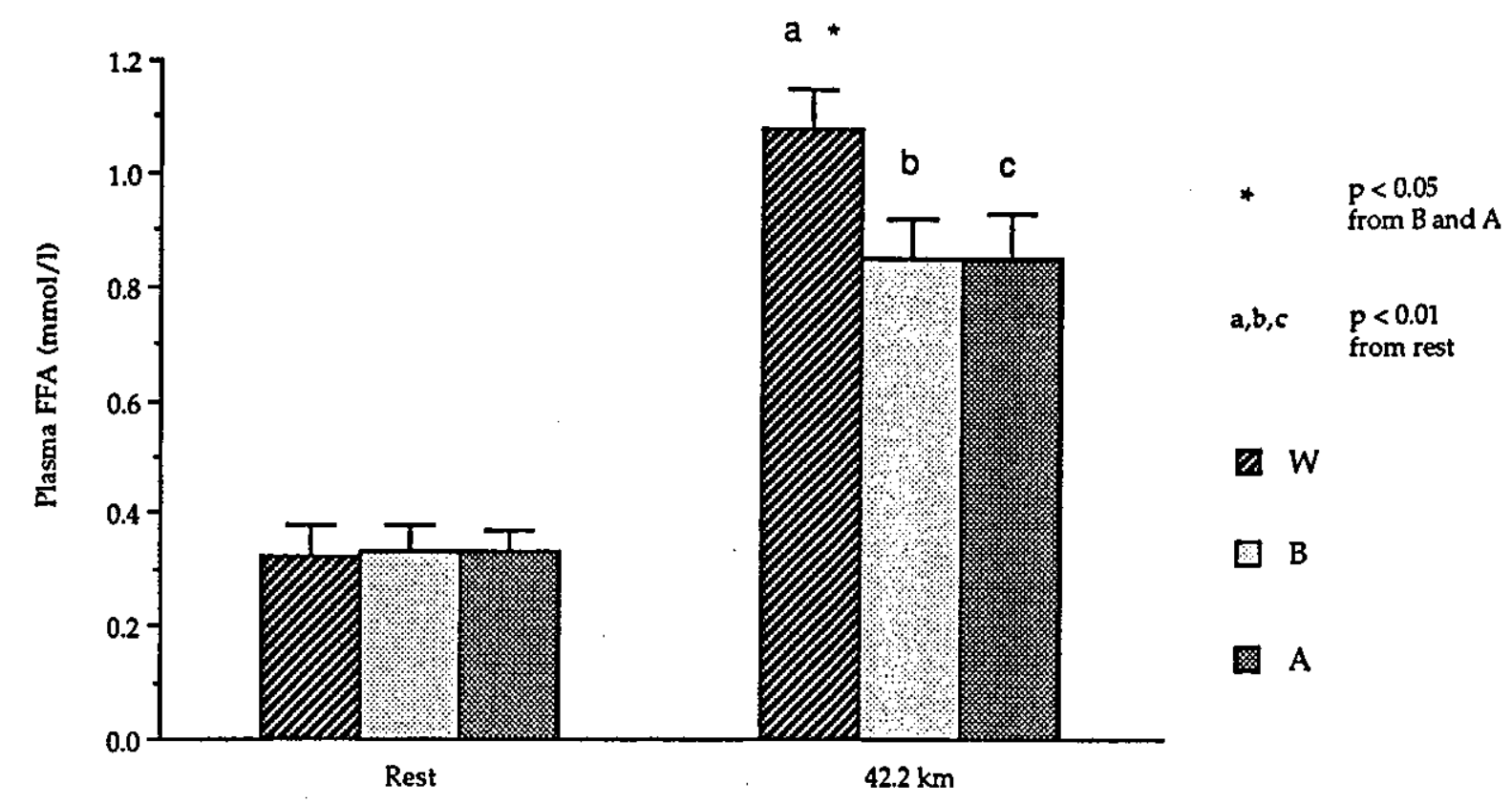

Fig. 4.6: Plasma FFA concentrations ( $(\mathrm{mmol} / \mathrm{d})$ for $W, B$ and $A$ trials during the $42.2-\mathrm{km}$ run (mean $\pm S E M, n=7$ )

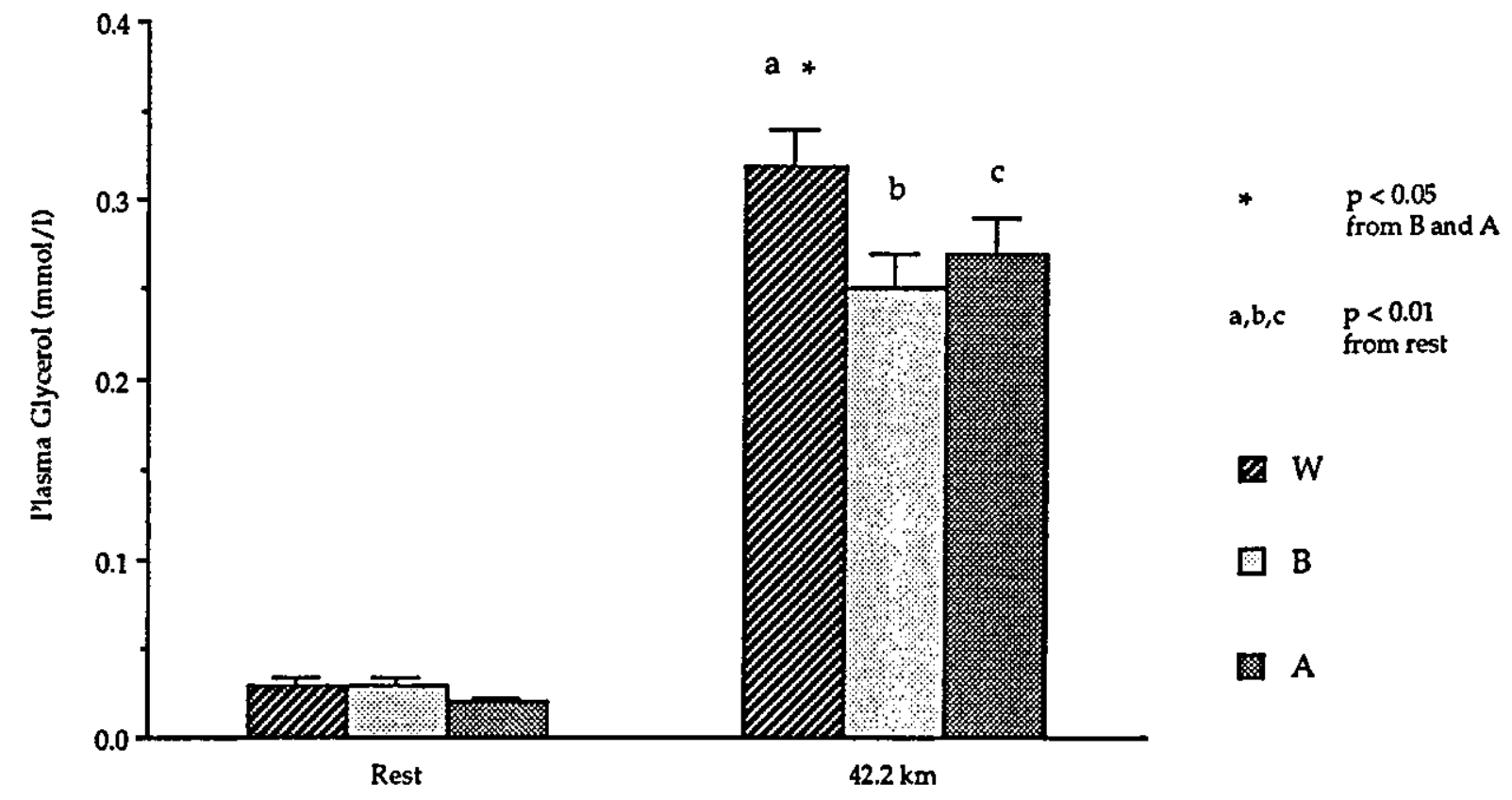

Fig. 4 7: Plasma glycerol concentrations (mmol/l) for $W, B$ and $A$ trials during the 42.2-km run (mean $\pm S E M, n=7$ ) 


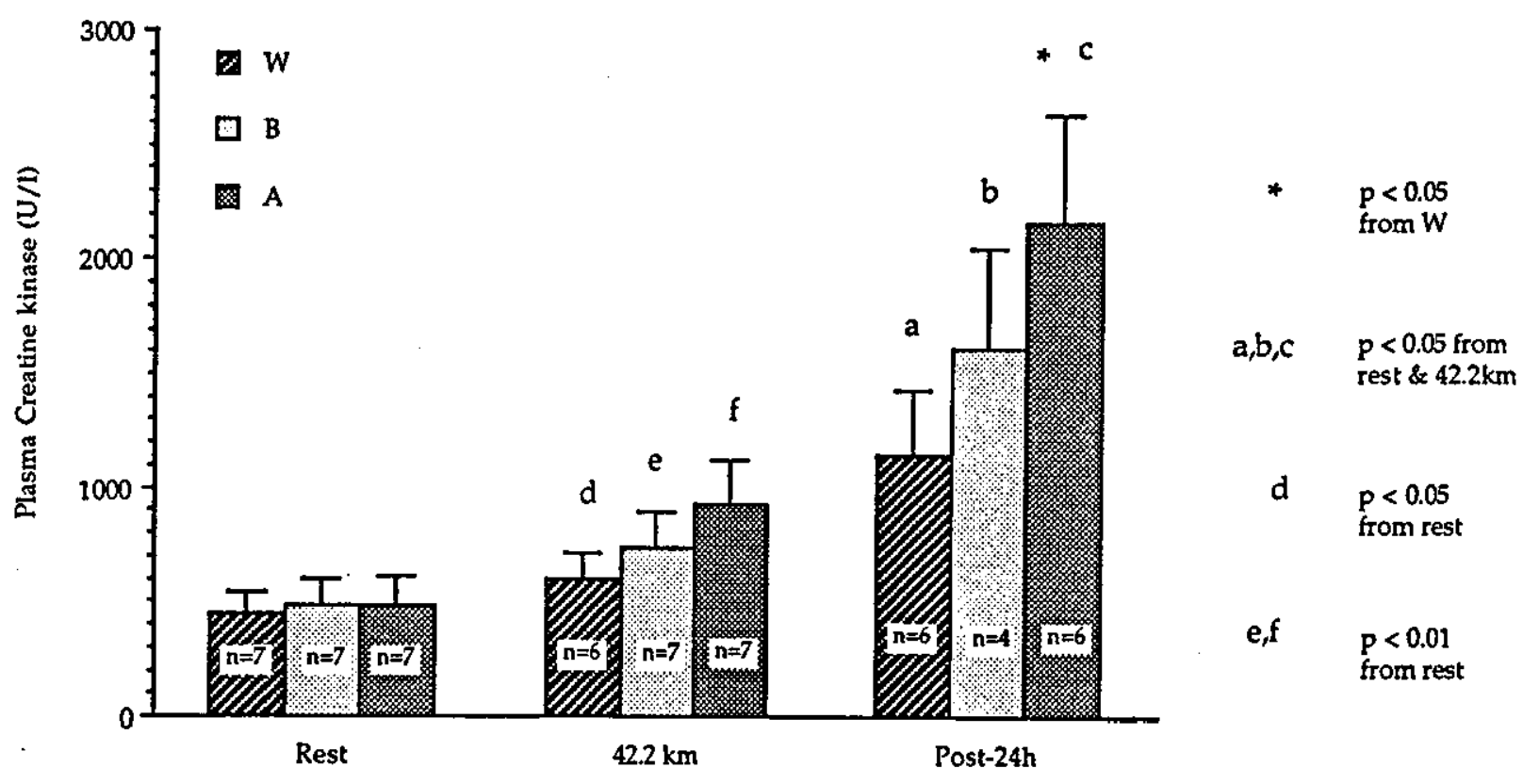

Fig. 4.8: Plasma creatine kinase concentrations (U/1) for W, B and A trials during the $42.2-\mathrm{km}$ run (mean \pm SEM)

Statistical analysis for the $B$ trial was performed for $n=4$. 


\subsection{DISCUSSION}

The main finding of this study was that drinking a 5.5\% carbohydrateelectrolyte solution throughout the marathon resulted in a better performance time than was achieved when the runners drank only water. No difference in performance times, however, was observed between the two carbohydrate trials, nor between the $B$ trial and the $W$ trial. In the $A$ trial the runners maintained their running speed for the whole of the marathon distance. In contrast, running speeds at the end of the $W$ and $B$ trials were significantly lower from 10 and $25 \mathrm{~km}$ of the run respectively. This result is consistent with previous studies from our laboratory for a 30$\mathrm{km}$ treadmill run (Williams et al. 1990) and a $30-\mathrm{km}$ road race (Tsintzas et al. 1993). The improvements in running performances were achieved as a result of drinking a 5\% carbohydrate-electrolyte solution which provided carbohydrate at a rate of $24 \mathrm{~g} \cdot \mathrm{h}^{-1}$, whereas in the present study the rate of carbohydrate ingestion was equivalent to $19 \mathrm{~g} \cdot \mathrm{h}^{-1}$. This is a low rate of $\mathrm{CHO}$ ingestion compared with most of the cycling studies which have shown improvements in endurance capacity. They have used $\mathrm{CHO}$ intakes that range from 22 to $111 \mathrm{~g}$. $\mathrm{h}^{-1}$ (Maughan et al. 1989; Fielding et al. 1985; Murray et al. 1991; Mitchell et al. 1989a; Coyle et al. 1986; Wright et al. 1991).

In a number of cycling studies, using similar exercise intensities and carbohydrate-electrolyte solutions, to those in present study, gastric emptying has been measured. Gastric emptying rates of between 7.7 and 8.2 ml.min ${ }^{-1}$ were reported in studies which used similar concentrations of CHO solutions, feeding frequencies ( $150 \mathrm{ml}$ every $15 \mathrm{~min}$ ) and exercise intensities (Houmard et al. 1991; Mitchell et al. 1989b). Assuming similar rates of gastric emptying in the present study, then virtually all of the carbohydrate-electrolyte solution would have been emptied during each marathon.

Studies using a wide range of concentrations of carbohydrate solutions during prolonged exercise have shown little differences in total and exogenous CHO oxidation rates (Rehrer et al. 1992; Maughan et. al. 1989). Thus irrespective of the concentration of the solution, only a part of the ingested $\mathrm{CHO}$ seems to be available for muscle metabolism. The excess $\mathrm{CHO}$ could be directed to and stored in the liver (Van Handel et al. 1980) 
and in non-exercising muscles (Rehrer et al. 1992) or even in non-active fibres of the exercising muscles (Kuipers et al. 1987).

One of the obvious explanations for the improvement in running performance reported in the present study is that the exogenous carbohydrate-electrolyte solution exerts a glycogen sparing effect in working muscles. This additional $\mathrm{CHO}$ complements the limited muscle glycogen stores and may extend exercise capacity by delaying glycogen depletion. There is support for glycogen-sparing from some (Bjorkman et al. 1984; Hargreaves et al. 1984) but not all cycling studies (Coyle et al. 1986; Coyle et al. 1991). Glycogen sparing, in the present study, would also explain the similar overall $\mathrm{CHO}$ oxidation rates found for all three trials. Valid comparisons of the $\mathrm{CHO}$ oxidation rates could not be made throughout the marathons because runners were free to choose their own running speeds. Nevertheless, they did maintain the same speeds for the first $5 \mathrm{~km}$ during each of the three marathons, during which there were no differences in the rates of $\mathrm{CHO}$ oxidation.

Although there were similar proportions of fat and carbohydrate oxidised during the three marathons, as suggested by RER values and similar changes in plasma insulin and adrenaline concentrations, plasma FFA and glycerol concentrations were lower in both $\mathrm{CHO}$ trials than in the $\mathrm{W}$ trial. It seems, therefore, that despite the decreased availability of plasma FFA, working muscles were able to oxidise a similar amount of fat in all three trials. Intra-muscular triglycerides are used during prolonged exercise and can cover a decrease in the supply of plasma fatty acids (Romijn et al 1993).

Under certain conditions, dehydration and the subsequent hyperthermia can decrease exercise capacity, well before muscle glycogen is depleted. In the present study, similar degrees of dehydration, changes in plasma volume, core temperatures and average sweat rates were observed in all three trials. Despite the relatively severe dehydration induced by the marathon in this study (5.5\% weight loss), core temperature was maintained within expected range $\left(36.6-38.9^{\circ} \mathrm{C}\right)$. This is consistent with previous studies (Pugh et al. 1967; Maughan et al. 1985; Myhre et al. 1985; Noakes et al. 1988). The rise in core temperature is directly related to the metabolic rate and thus running speed sustained during prolonged exercise (Maughan et al. 1985; Noakes et al. 1991a). Thus, the higher 
running speed and $\mathrm{V}_{2}$ observed at the end of the A trial compared with the $W$ trial generated a higher rate of heat production. Although rectal temperatures were the same in each trial, mean skin temperature was higher during the latter stages of the A trial compared with the values recorded for the $B$ and $W$ trials. Furthermore, because neither sweat rates nor changes in plasma volume were different between trials, an increased skin blood flow in the A trial would have accounted for the greater heat dissipation in that trial (Hamilton et al. 1991).

Fatigue occurs in skeletal muscle fibres when the total adenine nucleotide pool (TAN) is reduced to critically low levels. There is some evidence that the degradation of TAN may occur during prolonged submaximal exercise (Broberg and Sahlin, 1989). It has been suggested that continuing exercise when muscle glycogen concentrations are low leads to an increase in free ADP and AMP deaminase activity, which is responsible for the degradation of AMP. One indicator of increased AMP deaminase activity within the muscle cell is an increase in plasma ammonia $\left(\mathrm{NH}_{3}\right)$ concentration. Plasma $\mathrm{NH}_{3}$ concentration increases dramatically during prolonged exercise to exhaustion (Broberg and Sahlin, 1989). In the present study higher plasma $\mathrm{NH}_{3}$ concentrations might have been expected during the $\mathrm{W}$ trial because of the three trials, carbohydrate stores would have probably been lower during this trial. Surprisingly, plasma $\mathrm{NH}_{3}$ concentration was significantly lower in the $\mathrm{W}$ trial as compared with the A trial. The other source of $\mathrm{NH}_{3}$ is the oxidative metabolism of branched chain amino acids, which is an obligatory response to an increase in muscle metabolism (see Maclean et al. 1992). Therefore, the higher plasma $\mathrm{NH}_{3}$ concentration in the A trial may simply reflect an increased rate of muscle metabolism as a consequence of a higher running speed during the A trial compared with the $W$ and $B$ trials.

Skeletal muscle damage could also contribute to fatigue during marathon running. The release of creatine kinase $(\mathrm{CK})$ from skeletal muscle cell into the systemic circulation has been used to assess the extent of muscle damage during prolonged running (Apple et al. 1985; Rogers et al. 1985). Plasma CK concentrations peak 24 hours after marathon races; with values in the range of 800-3300 U.1-1 (Noakes, 1987; Rogers et al. 1985; HellstenWesting et al. 1991). In the present study plasma CK concentration was significantly higher 24 hours after the A trial, as compared with the $\mathrm{W}$ trial 
$\left(2164 \pm 469\right.$ U.1 $1^{-1}$ vs $1146 \pm 280$ U.1.1 $)$. The higher exercise intensity sustained towards the latter stages of the $A$ trial was probably responsible for the greater increase in plasma $C K$ concentration. Higher running speeds demand a greater muscle fibre recruitment which could lead to proportionally greater muscle damage and $\mathrm{CK}$ release into the blood (Rogers et al. 1985).

It is not clear, however, why the ingestion of the B solution was not equally effective in maintaining running speed after the first $25 \mathrm{~km}$ of the run. Differences in $\mathrm{CHO}$ concentration and composition, palatability and flavour between the two drinks could have contributed in some way to difference in performance or rather the lack of difference in performance between the $B$ drink and water. Interestingly, 6 out of the 7 runners in this study expressed their preference for the A solution, when compared with the $B$ solution, in terms of taste and palatability. In addition, three cases of gastrointestinal disturbance were observed during the $B$ trial compared with only one in the A trial. However, the reported gastrointestinal disturbances did not cause runners to decrease their running speeds during the treadmill time trials.

In summary, the ingestion of $\mathrm{A}(5.5 \% \mathrm{CHO})$ solution in comparison with the ingestion of the same volume of water before and during a $42-\mathrm{km}$ treadmill run improved endurance performance. Runners were able to maintain their running speeds for the whole of the marathon when drinking the A solution, which was not the case when they consumed either the B solution or water. 


\section{CHAPTER 5}

\section{INFLUENCE OF CARBOHYDRATE INGESTION ON RUNNING ENDURANCE CAPACITY}

\subsection{INTRODUCTION}

Carbohydrate ingestion either throughout or late in exercise has been shown to improve endurance capacity during prolonged cycling exercise of moderate intensity (Coyle et al. 1983; 1986; Coggan and Coyle, 1989). It has been suggested by those researchers that carbohydrate ingestion delays the onset of fatigue by simply preventing the decline in blood glucose concentration and maintaining carbohydrate oxidation at a high rate late in exercise rather than sparing muscle glycogen.

During prolonged running exercise without $\mathrm{CHO}$ supplementation, however, blood glucose and total $\mathrm{CHO}$ oxidation rate do not appear to decline prior to exhaustion. Yet, endurance performance has been shown to improve when $\mathrm{CHO}$ is ingested during this type of exercise. Indeed, in our previous studies carbohydrate as opposed to water ingestion during either a $30-\mathrm{km}$ road race (Tsintzas et al. 1993) or a $42.2-\mathrm{km}$ treadmill time trial (see Chapter 4) resulted in an improved endurance performance. Moreover, $\mathrm{CHO}$ ingestion did not affect total $\mathrm{CHO}$ oxidation rates. It was hypothesized, therefore, that if sparing of muscle glycogen is the mechanism underlying the ergogenic effect of $\mathrm{CHO}$ ingestion during running exercise, then $\mathrm{CHO}$ ingestion restricted to the early part of prolonged exercise should also enhance endurance capacity.

The aim of the present investigation, therefore, was to examine whether or not carbohydrate supplementation during the first hour of treadmill running at constant speed delays the onset of fatigue. 


\subsection{METHODS}

Subjects: Eleven male recreational runners volunteered to participate in this study. Nine subjects completed all three trials and two completed only two trials. The mean age, height, weight, $\mathrm{VO}_{2}$ max and $\mathrm{HR}$ max of the subjects were $27.0( \pm 1.9)$ years $( \pm \mathrm{SEM}), 174.7( \pm 2.2) \mathrm{cm}, 71.0( \pm 3.1) \mathrm{kg}, 61.7( \pm 1.8)$ $\mathrm{ml} . \mathrm{kg}^{-1} \cdot \mathrm{min}^{-1}$ and $193.5( \pm 2.5)$ bt.min ${ }^{-1}$, respectively.

Preliminary measurements: Following familiarization with treadmill running and experimental procedures, the subjects undertook three preliminary tests (see Chapter 3 ) in order to determine: (i) the relationship between running speed and oxygen uptake, (ii) the maximum oxygen uptake ( $\mathrm{VO}_{2}$ max), and (iii) the running speed and percentage $\mathrm{VO}_{2}$ max equivalent

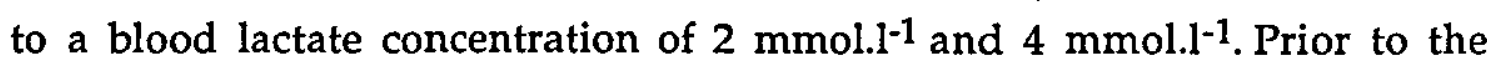
first experimental trial, the subjects undertook a 60 -min treadmill run at $70 \%$ $\mathrm{VO}_{2}$ max in order to fully familiarized themselves with the drinking pattern and the measurements used during the experimental trials. There was no order effect for the three runs to exhaustion (trial 1: $122.3 \pm 10.2 \mathrm{~min}$, trial 2: $122.8 \pm 9.0 \mathrm{~min}$, trial 3: $117.6 \pm 11.9 \mathrm{~min}$ ). A balanced randomization of the trials was applied for the nine subjects who completed all three runs (three subjects per treatment per trial).

Protocol of the study: The subjects were required to run to exhaustion at $70 \%$ $\mathrm{VO}_{2}$ max on a motorized treadmill on three different occasions separated by one week (Fig. 5.1). On the first and second occasions two isotonic carbohydrate-electrolyte solutions (a 5.5\% (A) and a $6.9 \%$ (C) solutions) were fed for the first hour of exercise, water was then fed until exhaustion. On the third occasion tap water (W) was fed throughout the run. The order of testing was randomly assigned and the experiment was conducted in a double-blind design with respect to $\mathrm{CHO}$ solutions.

All trials took place following a two-day normal standardised diet and a 10-12 hour overnight fast. A warm-up which consisted of 5-min run at $60 \% \mathrm{VO}_{2}$ max preceded each trial. The treadmill was then stopped for $5 \mathrm{~min}$ and the subject consumed $8 \mathrm{ml} . \mathrm{kg}^{-1}$ body weight (bw) of the assigned fluid while standing on the treadmill. A further $2 \mathrm{ml}^{\mathrm{kg}} \mathrm{kg}^{-1} \mathrm{bw}$ of the assigned fluid was ingested by the subjects every $20 \mathrm{~min}$ of the run. Immediately after this 5 min period, the treadmill running speed was brought up to $70 \% \mathrm{VO}_{2}$ max. The treadmill was stopped for $3 \mathrm{~min}$ at $60 \mathrm{~min}$ of the run. The subject simply 
remained in a standing position before he resumed running at $70 \% \mathrm{VO}_{2}$ max until exhaustion. The reason for this brief stop was that in a subsequent study using the same protocol muscle biopsy samples were obtained at this point of time. Blood samples were obtained at rest, every $20 \mathrm{~min}$ of the run and at exhaustion. One minute expired air samples were collected every 20 min and during the last min of the run. The Borg scale of perceived exertion (Borg, 1973) and a simple 1-10 scale of perceived thirst were also used at the same intervals. Following expired air and blood sampling, the assigned fluid was ingested by the subjects every $20 \mathrm{~min}$ of the run. Wet sponges were available to the subjects to be used ad libitum throughout the runs. Heart rate and skin and rectal temperatures were continuously monitored throughout the run. The subject's pre-exercise and post-exercise nude body weight was also obtained.

Dry bulb temperatures within the laboratory were $19.6 \pm 0.2{ }^{\circ} \mathrm{C}, 19.5 \pm 0.2{ }^{\circ} \mathrm{C}$ and $19.6 \pm 0.1{ }^{\circ} \mathrm{C}$ during the $\mathrm{W}, \mathrm{A}$ and $\mathrm{C}$ trials respectively. Relative humidity for the three conditions was $51.4 \pm 2.5 \%, 54.7 \pm 2.2 \%$ and $51.8 \pm 3.1 \%$ respectively.

Analysis: Expired air samples were collected and analysed as previously described (see Chapter 3). Venous blood samples were analysed for: blood lactate, glucose and haemoglobin concentrations and haematocrit values; plasma ammonia, sodium, potassium, FFA, glycerol, adrenaline and noradrenaline concentrations; and serum insulin, cortisol and growth hormone concentrations. Percentage changes in plasma volume were measured as previously described (see Chapter 3 ).

Statistical analysis: A two-way analysis of variance (ANOVA) for repeated measures on two factors (experimental treatment and sampling time) was used to compare differences between cardiovascular changes and blood metabolites for all trials of this study. Times to exhaustion, body weight changes and volumes of fluid ingested during the three trials were analysed by one-way ANOVA for correlated data. When using ANOVA any significant differences shown were assessed by applying the Tukey post-hoc test. Statistical significance was accepted at $5 \%$ level. Results are presented as mean \pm SEM. 
Fluid ingestions

Heart rate and body temperature measurements

कै
$8 \mathrm{ml} / \mathrm{kg} \mathrm{BW}$

$\downarrow$
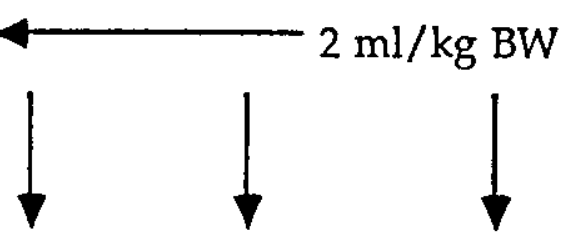
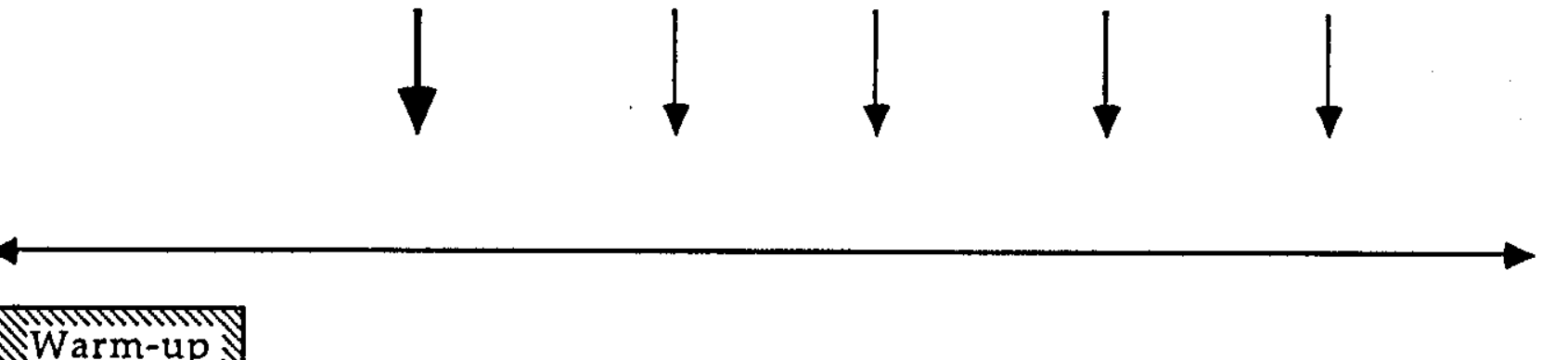

Warm-up

5 min at $60 \%$

5 min at $60 \%$
$V O 2 \max$
rest

rest Running at $70 \% \mathrm{VO} 2 \mathrm{max} \quad 3 \mathrm{~min}$ rest

$70 \% \mathrm{VO} 2 \max$

60

$\begin{array}{lllll}10 & 20 & 30 & 40 & 50\end{array}$

Time (min)

80

Start
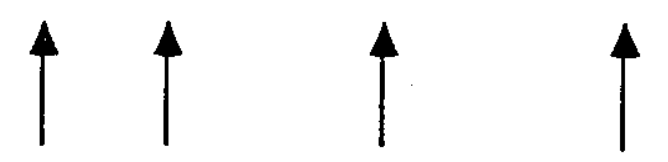

Exhaustion

Venous blood samples

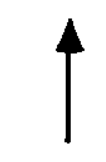

Expired air samples
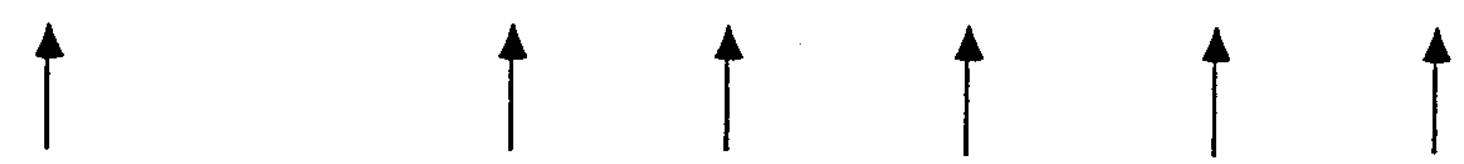

Fig.5.1: Schematic illustration of the study's protocol 


\subsection{RESULTS}

Performance times for the $W, A^{S}$ and $C^{6}$ trials were $109.6 \pm 9.6 \mathrm{~min}(n=11)$, $124.5 \pm 8.4 \mathrm{~min}(n=10)$ and $121.4 \pm 9.4(n=10) \mathrm{min}$, respectively (Fig. 5.2). Performance time for the A trial was significantly longer $(p<0.05)$ compared with the $W$ trial. No significant difference was found between either the $W$ and $\mathrm{C}$ or the two $\mathrm{CHO}$ trials. Times to exhaustion for all three trials were not correlated with $\mathrm{VO}_{2}$ max values; nor they were correlated with running speed (m. $\left.\mathrm{s}^{-1}\right)$ and $\% \mathrm{VO}_{2}$ max equivalent to blood lactic acid concentrations of $2 \mathrm{mmol.1} \mathrm{l}^{-1}$ or $4 \mathrm{mmoll}^{-1}$.

No difference was observed between the $\mathrm{W}, \mathrm{A}$ and $\mathrm{C}$ trials for total volume of fluid ingested during the runs ( $1229 \pm 93 \mathrm{ml}$ vs $1372 \pm 93 \mathrm{ml}$ vs $1311 \pm 108$ $\mathrm{ml}$, respectively). In the $A$ and $C$ trials $869 \pm 36 \mathrm{ml}$ and $852 \pm 42 \mathrm{ml}$, respectively, of the total fluid was ingested as $\mathrm{CHO}$ solution during the first hour of the run. As a result, a total of $47.8 \pm 2.0 \mathrm{~g}$ and $58.8 \pm 2.9 \mathrm{~g}$ of CHO was ingested during the same period of time.

The average $\% \mathrm{VO}_{2}$ max sustained during the $\mathrm{W}, \mathrm{A}$ and $\mathrm{C}$ trials was not different between the three trials $(70.2 \pm 1.0 \%$ vs $70.5 \pm 1.1 \%$ vs $70.9 \pm 1.4$ respectively). Neither was there any difference between trials in oxygen uptake $\left(\mathrm{VO}_{2}\right)$ and, hence, \%VO $2 \max$ at the various sampling points (Table 5.1). Respiratory Exchange Ratio (RER) was also not different between trials, except for the 80 min where RER was significantly higher $(p<0.05)$ in the $C$ trial compared with the $W$ trial (Table 5.1). Total carbohydrate oxidation rate, as calculated from $\mathrm{VO}_{2}$ and RER values, was similar at all points in the $\mathrm{W}$ and $\mathrm{A}$ trials. On the other hand, higher $\mathrm{CHO}$ oxidation rate was observed at $20 \mathrm{~min}, 40 \mathrm{~min}, 60 \mathrm{~min}$ and $80 \mathrm{~min}$ of exercise in the $C$ trial compared with the $\mathrm{W}$ trial. However, $\mathrm{CHO}$ oxidation rate was similar at exhaustion in all three trials (Table 5.1).

Similar mean heart rate was observed in the $W, A$ and $C$ trials $(166 \pm 2$ beats. min $^{-1}$ vs $164 \pm 2$ beats. min $^{-1}$ vs $168 \pm 2$ beats.min ${ }^{-1}$ respectively). Furthermore, as can be seen in Table 5.1, heart rate was not different at any sampling point between the three trials.

No difference was observed in mean skin temperature $\left(30.6 \pm 0.4{ }^{\circ} \mathrm{C}\right.$ vs $30.7 \pm$ $0.4^{\circ} \mathrm{C}$ vs $\left.30.3 \pm 0.4^{\circ} \mathrm{C}\right)$, rectal temperature at exhaustion $\left(39.1 \pm 0.3^{\circ} \mathrm{C}\right.$ vs 39.0 
$\pm 0.2{ }^{\circ} \mathrm{C}$ vs $39.2 \pm 0.4{ }^{\circ} \mathrm{C}$ ), body weight loss corrected for fluid ingested (2.8 \pm $0.3 \mathrm{~kg}$ vs $3.0 \pm 0.2 \mathrm{~kg}$ vs $2.9 \pm 0.3 \mathrm{~kg}$ ) and percentage change in plasma volume $(-1.3 \pm 2.3 \%$ vs $-3.3 \pm 2.4 \%$ vs $-5.2 \pm 1.7 \%)$ between the $\mathrm{W}, \mathrm{A}$ and $\mathrm{C}$ trials, respectively. Perceived rate of exertion and thirst were also not different between trials. It should be pointed out, however, that three cases of gastrointestinal discomfort during the run were recorded in each of the $W$ and $\mathrm{A}$ trials. In contrast, seven cases were observed during the $C$ trial.

Higher blood glucose concentration $(\mathrm{p}<0.01)$ was observed at $20 \mathrm{~min}$ in the A trial compared with the $W$ trial $\left(4.7 \pm 0.4 \mathrm{mmol}^{-1}\right.$ vs $3.7 \pm 0.2 \mathrm{mmol.1} \mathrm{l}^{-1}$ respectively) (Fig. 5.3). No significant difference was found in blood glucose concentration at any other point of time, including exhaustion, between the three trials. However, lower blood glucose concentration $(p<0.01)$ was observed at $80 \mathrm{~min}$ in all three trials compared with $60 \mathrm{~min}$. Interestingly, there was no decline in blood glucose concentration at the end of the $\mathrm{W}$ trial (Fig. 5.3).

Blood lactate (Fig. 5.4), plasma ammonia, serum insulin, serum cortisol, plasma adrenaline and plasma nor-adrenaline concentrations were also not significantly different between the three trials (Table 5.2). When compared with resting values, plasma ammonia concentration was higher $(p<0.01)$ at $60 \mathrm{~min}$ of the run in all three trials. No further increase was observed until exhaustion in the $W$ and $A$ trials. However, an increase $(p<0.01)$ was found at exhaustion compared with $60 \mathrm{~min}$ in the $\mathrm{C}$ trial. Plasma cortisol concentration was not elevated at $60 \mathrm{~min}$ compared with rest; at exhaustion, however, it was higher ( $p<0.01$ ) from $60 \mathrm{~min}$ in all three trials. Lower plasma insulin concentrations were observed at the end of all three trials compared with rest $(p<0.01)$. Plasma adrenaline concentrations were higher at exhaustion ( $p<0.01$ and $p<0.05$ ) but not at $60 \mathrm{~min}$, whereas plasma noradrenaline concentrations were higher $(p<0.01)$ both at $60 \mathrm{~min}$ and exhaustion compared with pre-exrcise values in all three trials.

On the other hand, serum growth hormone (Table 5.2), plasma FFA (Fig. 5.5) and plasma glycerol (Fig. 5.6) concentrations were significantly higher ( $\mathrm{p}<$ 0.05 ) at $60 \mathrm{~min}$ in the $\mathrm{W}$ trial compared with both the $\mathrm{CHO}$ trials. Serum $\mathrm{GH}$ and plasma FFA concentrations were not different from resting levels at 60 min of exercise in both the $\mathrm{CHO}$ trials. The replacement of $\mathrm{CHO}$ ingestion with water ingestion after this point of time provoked a dramatic increase in 
the concentration of these metabolites. As a result, there was no difference in the concentration of GH and FFA at exhaustion between the three trials. Plasma glycerol concentration was also not different at exhaustion between trials.

Plasma electrolyte concentrations are shown in Table 5.3. Plasma sodium concentrations did not change over time during the $\mathrm{W}, \mathrm{A}$ and $\mathrm{C}$ trials and at the point of exhaustion were similar in all three trials ( $139 \pm 1 \mathrm{mmol} . \mathrm{l}^{-1} \mathrm{vs}$ $138 \pm 1 \mathrm{mmol}^{-1}$ vs $140 \pm 1 \mathrm{mmol.1}-1$, respectively). On the other hand, a significant rise in plasma potassium concentration was observed at $20 \mathrm{~min}$ of the run in all three trials compared with rest $(p<0.01)$. Plasma potassium concentration remained elevated until exhaustion in all three trials. However, no difference was observed between trials at any sampling time. 
Table 5.1: Oxygen uptake $\left(\mathrm{VO}_{2}\right)\left(1 . \mathrm{min}^{-1}\right), \% \mathrm{VO}_{2}$ max, Respiratory Exchange Ratio (RER), CHO oxidation rate (CHO-OR) (g.min ${ }^{-1}$ ) and Heart Rate (HR) (bts.min ${ }^{-1}$ ) during the W, A and $C$ trials (mean $\pm S E M, n=11$ )

Variables

\begin{tabular}{|c|c|c|c|c|c|c|}
\hline $\mathrm{VO}_{2}$ & W & 3.0 & 3.0 & 3.1 & 3.0 & $3.2^{\mathrm{a}}$ \\
\hline & & \pm 0.1 & \pm 0.1 & \pm 0.1 & \pm 0.2 & \pm 0.1 \\
\hline & A & 3.1 & 3.1 & 3.0 & 3.1 & $3.2^{b}$ \\
\hline & & \pm 0.1 & \pm 0.1 & \pm 0.1 & \pm 0.1 & \pm 0.1 \\
\hline & $C$ & 3.1 & 3.1 & 3.1 & 3.1 & 3.2 \\
\hline & & \pm 0.1 & \pm 0.1 & \pm 0.1 & \pm 0.1 & \pm 0.2 \\
\hline$\% \mathrm{VO}_{2} \max$ & W & 69.6 & 69.7 & 70.8 & 70.1 & $72.6^{a}$ \\
\hline & & \pm 0.6 & \pm 1.0 & \pm 1.0 & \pm 1.0 & \pm 1.0 \\
\hline & A & 70.1 & 70.4 & 69.0 & 70.6 & $72.5^{\mathrm{b}}$ \\
\hline & & \pm 0.8 & \pm 0.8 & \pm 1.2 & \pm 0.8 & \pm 1.6 \\
\hline & C & 70.8 & 70.7 & 70.5 & 70.4 & 72.6 \\
\hline & & \pm 1.2 & \pm 1.4 & \pm 1.1 & \pm 1.2 & \pm 1.4 \\
\hline RER & W & 0.90 & 0.90 & 0.89 & 0.90 & 0.89 \\
\hline & & \pm 0.01 & \pm 0.01 & \pm 0.01 & \pm 0.01 & \pm 0.01 \\
\hline & A & 0.91 & 0.91 & 0.90 & 0.91 & 0.90 \\
\hline & & \pm 0.01 & \pm 0.01 & \pm 0.01 & \pm 0.01 & \pm 0.01 \\
\hline & C & 0.93 & 0.93 & 0.93 & $0.94^{*}$ & 0.91 \\
\hline & & \pm 0.01 & \pm 0.01 & \pm 0.01 & \pm 0.01 & \pm 0.01 \\
\hline CHO-OR & W & 2.35 & 2.41 & 2.34 & 2.37 & 2.35 \\
\hline & & \pm 0.15 & \pm 0.17 & \pm 0.12 & \pm 0.18 & \pm 0.18 \\
\hline & A & 2.55 & 2.51 & 2.43 & 2.57 & 2.46 \\
\hline & & \pm 0.15 & \pm 0.12 & \pm 0.13 & \pm 0.11 & \pm 0.1 \\
\hline & C & $2.76^{*}$ & $2.77^{*}$ & $2.72^{\mathrm{C}}$ & $2.91^{* *}$ & 2.60 \\
\hline & & \pm 0.14 & \pm 0.16 & \pm 0.14 & \pm 0.14 & \pm 0.23 \\
\hline HR & W & 162 & 167 & 169 & 167 & $176^{d}$ \\
\hline & & \pm 4 & \pm 5 & \pm 6 & \pm 6 & \pm \\
\hline & A & 159 & 163 & 164 & 168 & $174^{\mathrm{e}}$ \\
\hline & & \pm 3 & \pm 4 & \pm 4 & \pm 4 & \pm \\
\hline & $C$ & 165 & 170 & 168 & 170 & $178^{f}$ \\
\hline & & \pm 4 & \pm 5 & \pm 5 & \pm 5 & \pm 4 \\
\hline
\end{tabular}

$\underline{20-\min } \quad \underline{40-\mathrm{min}} \quad \underline{60-\mathrm{min}} \quad \underline{80-\mathrm{min}} \quad$ Exhaustion

.46

0.14

2.60

$176^{\mathrm{d}}$

$\pm 4$

$74^{\mathrm{e}}$

$178^{\mathrm{f}}$

$\pm 4$

\footnotetext{
* $\quad \mathrm{p}<0.05$ from $W$

** $\quad p<0.05$ from $W$ and $A$

a $\quad \mathrm{p}<0.05$ from $20 \mathrm{~min}$

b $\quad p<0.05$ from $60 \mathrm{~min}$

c $\quad p=0.05$ from $W$

$\mathrm{d}, \mathrm{e}, \mathrm{f} \quad \mathrm{p}<0.01$ from $20 \mathrm{~min}$
} 
Table 5.2: Plasma ammonia $\left(\mu m o l .1^{-1}\right)$, serum insulin $\left(m U .1^{-1}\right)$, serum cortisol (nmol..$\left.^{-1}\right)$, serum growth hormone $(\mathrm{GH})\left(\mathrm{mU} . \mathrm{l}^{-1}\right)$, plasma adrenaline (nmol. ${ }^{-1}$ ) and plasma nor-adrenaline (nmol. $1^{-1}$ ) concentrations for $W, A$ and $C$ trials during the run to exhaustion (mean $\pm S E M$, $\mathrm{n}=11$ )

Metabolites

Plasma ammonia $\quad$ W

A

C

Serum insulin

Serum cortisol

W

C

Serum $\mathrm{GH}$

Plasma

nor-adrenaline

W

A

C

A

C

Plasma adrenaline

A
Rest

$38.9 \pm 4.0$.

$29.9 \pm 4.1$

$31.9 \pm 3.5$

W
C

W

$5.4 \pm 0.6$

$5.2 \pm 1.0$

$5.4 \pm 0.5$

$619 \pm 34$

$653 \pm 42$

$602 \pm 31$

$6.7 \pm 2.4$
$2.3 \pm 0.7$
$1.5 \pm 0.4$

$0.59 \pm 0.28$

$0.24 \pm 0.04$

$0.20 \pm 0.04$

60-min

$95.7 \pm 13.7^{a}$

$98.0 \pm 11.5^{\mathrm{a}}$

$100.4 \pm 9.8^{\mathrm{a}}$

$3.2 \pm 0.8^{d}$

$3.8 \pm 0.5$

$4.5 \pm 0.6$

$561 \pm 68$

$567 \pm 74$

$576 \pm 55$

$27.4 \pm 6.4^{*, a}$

$10.6 \pm 2.1$

$11.0 \pm 3.1$

$1 \pm 7.1^{a}$

$32.8 \pm 6.1^{a, b}$

$34.8 \pm 5.0^{\mathrm{a}, \mathrm{b}}$

$2.38 \pm 0.42 \mathrm{c}, \mathrm{d}$

$3.81 \pm 0.94 \mathrm{a}, \mathrm{b}$

$3.45 \pm 0.66 \mathrm{a}, \mathrm{b}$

$1.00 \pm 0.41$

$0.78 \pm 0.23 \mathrm{~d}$

$2.56 \pm 0.27$

$13.19 \pm 2.19 a$

$17.48 \pm 2.70^{\mathrm{a}}$

$2.64 \pm 0.47$

$11.18 \pm 1.92^{\mathrm{a}}$

$12.94 \pm 1.21^{\mathrm{a}}$

C

$2.35 \pm 0.31$

$11.46 \pm 1.08 \mathrm{a}$

$15.49 \pm 2.50^{\mathrm{a}}$ 
Table 5.3: Plasma sodium and potassium concentrations (mmol. $1^{-1}$ ) for $W, A$ and $C$ trials during the run to exhaustion (mean $\pm S E M, n=11$ )

Metabolites

Plasma sodium

W

A

C

Plasma potassium

W

A

C

* $\mathrm{p}<0.01$ from Rest
Rest 20-min $\underline{60-\mathrm{min}}$ Exhaustion

$\begin{array}{llll}138 \pm 1 & 137 \pm 1 & 138 \pm 1 & 139 \pm 1 \\ 138 \pm 1 & 139 \pm 1 & 138 \pm 1 & 138 \pm 1 \\ 138 \pm 1 & 139 \pm 1 & 140 \pm 1 & 140 \pm 1\end{array}$

$3.9 \pm 0$

$4.9 \pm 0.1^{*}$

$4.7 \pm 0.1^{*}$

$4.7 \pm 0.1^{*}$

$3.9 \pm 0.1$

$4.8 \pm 0.1^{*}$

$4.7 \pm 0.1 *$

$4.5 \pm 0.1^{*}$

$3.8 \pm 0.1$

$4.9 \pm 0.1^{*}$

$4.7 \pm 0.1^{*}$

$4.8 \pm 0.2^{*}$ 


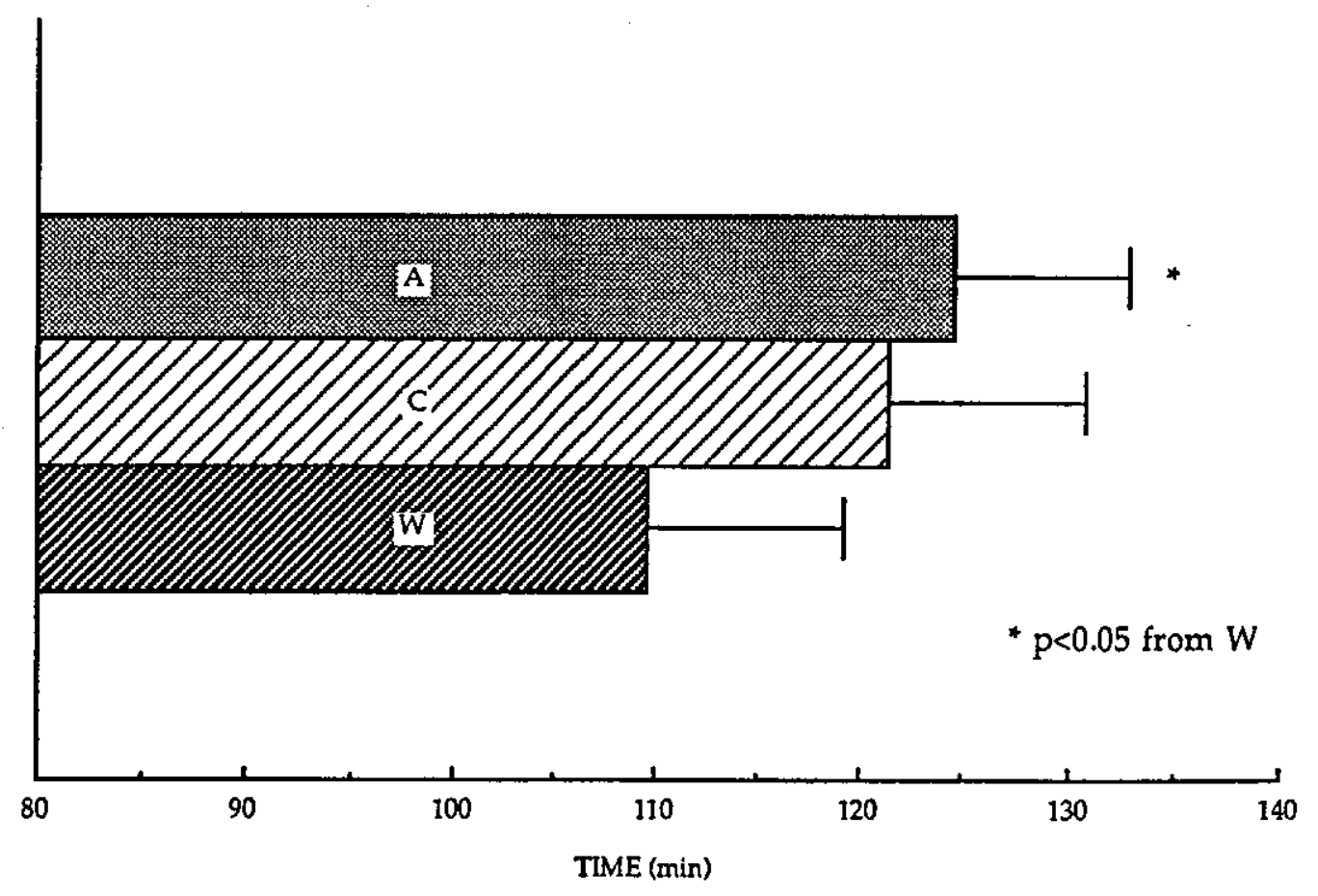

Fig. 5.2: Time to exhaustion ( $m i n)$ for the $W(n=11), A(n=10)$ and $C(n=10)$ trials (mean $\pm \mathrm{SEM}$ ) 


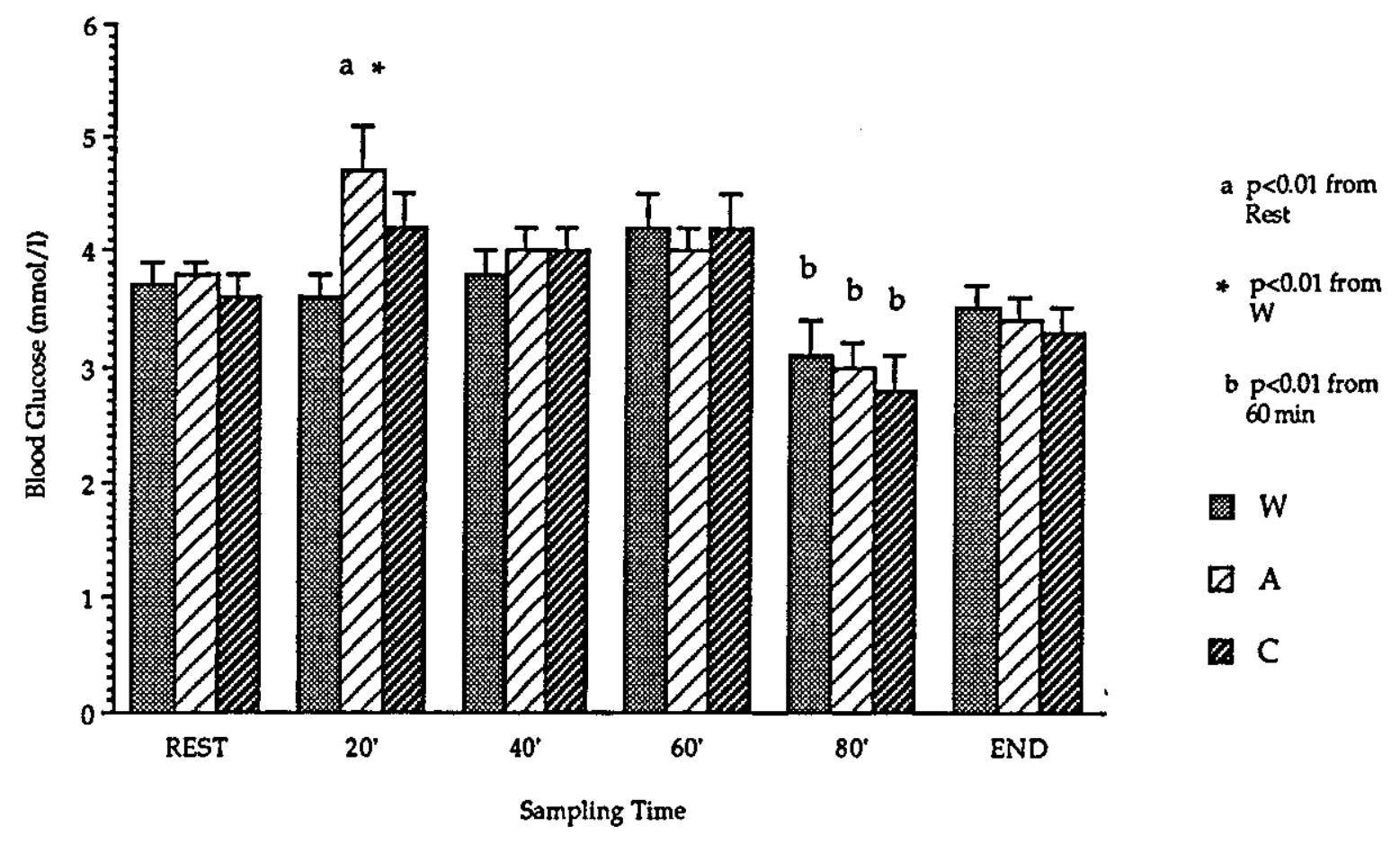

Fig. 5.3: Blood glucose concentrations (mmol/l) for the $W, A$ and $C$ trials during the run to exhaustion (mean $\pm S E M, n=11$ )

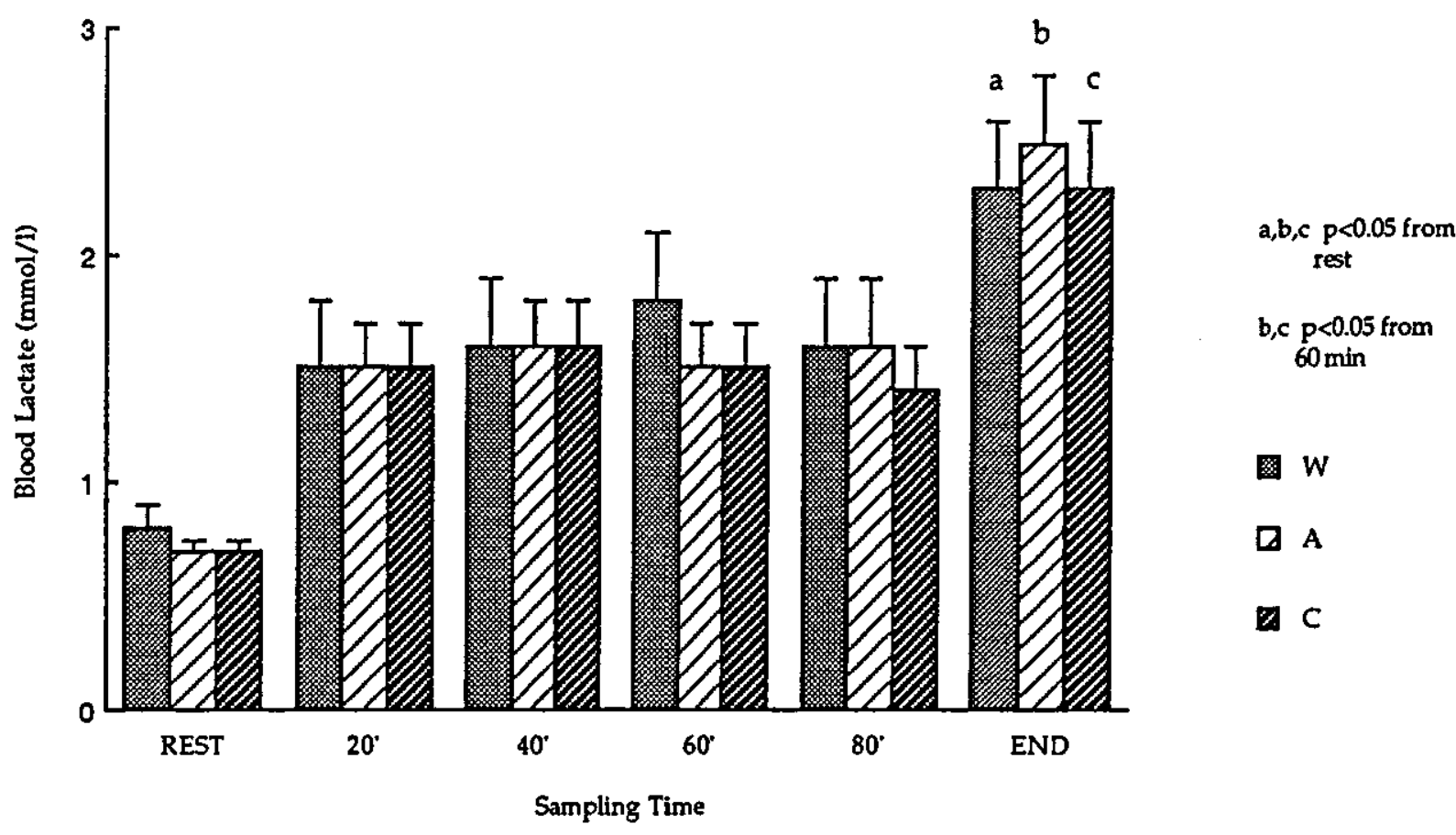

Fig. 5.4: Blood Lactate concentration (mmol/l) for $W, A$ and $C$ trials (mean $\pm S E M, n=11$ ) 


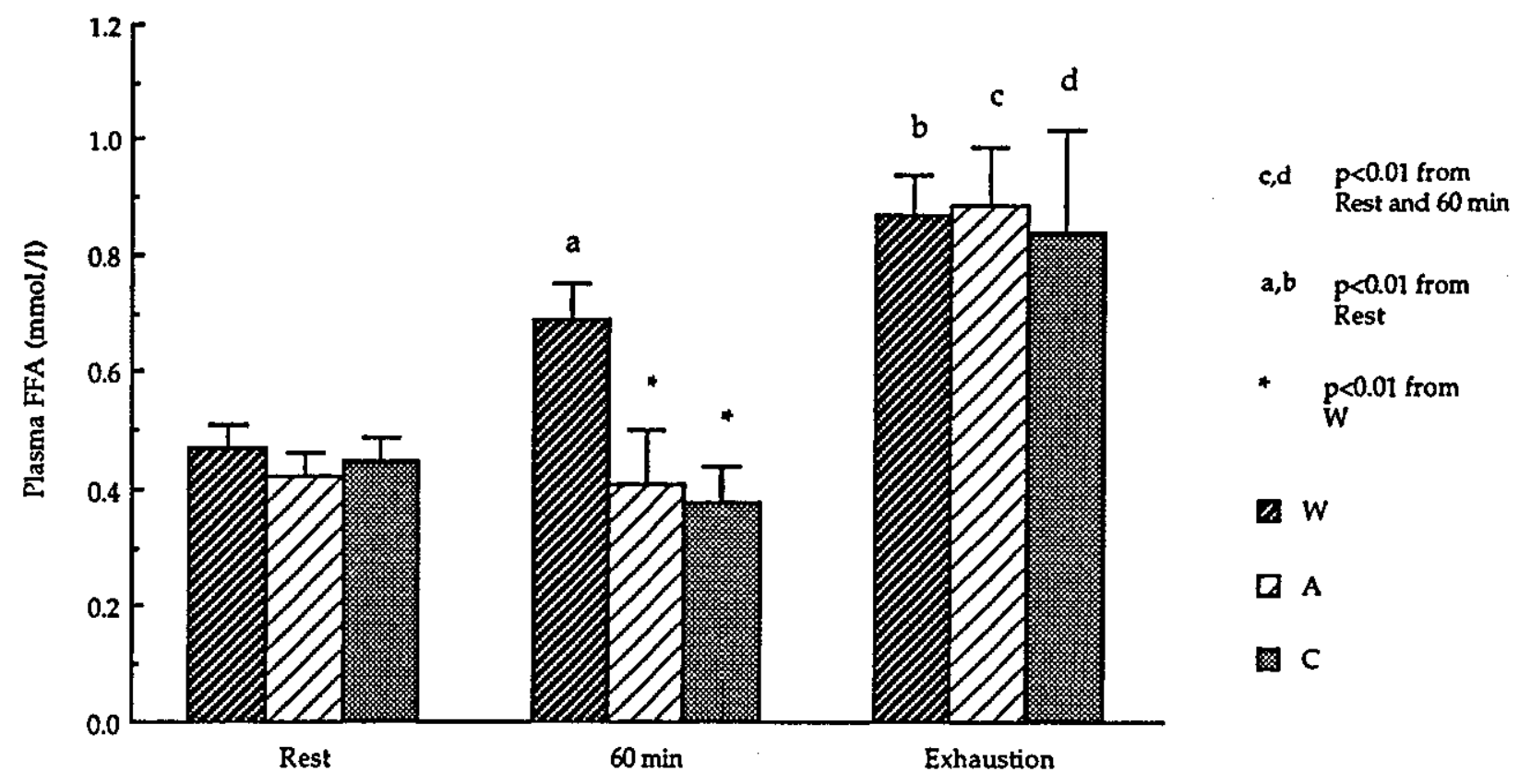

Fig. 5.5: Plasma FFA concentrations (mmol/1) during the $W, A$ and $C$ trials (mean $\pm S E M, n=11$ )

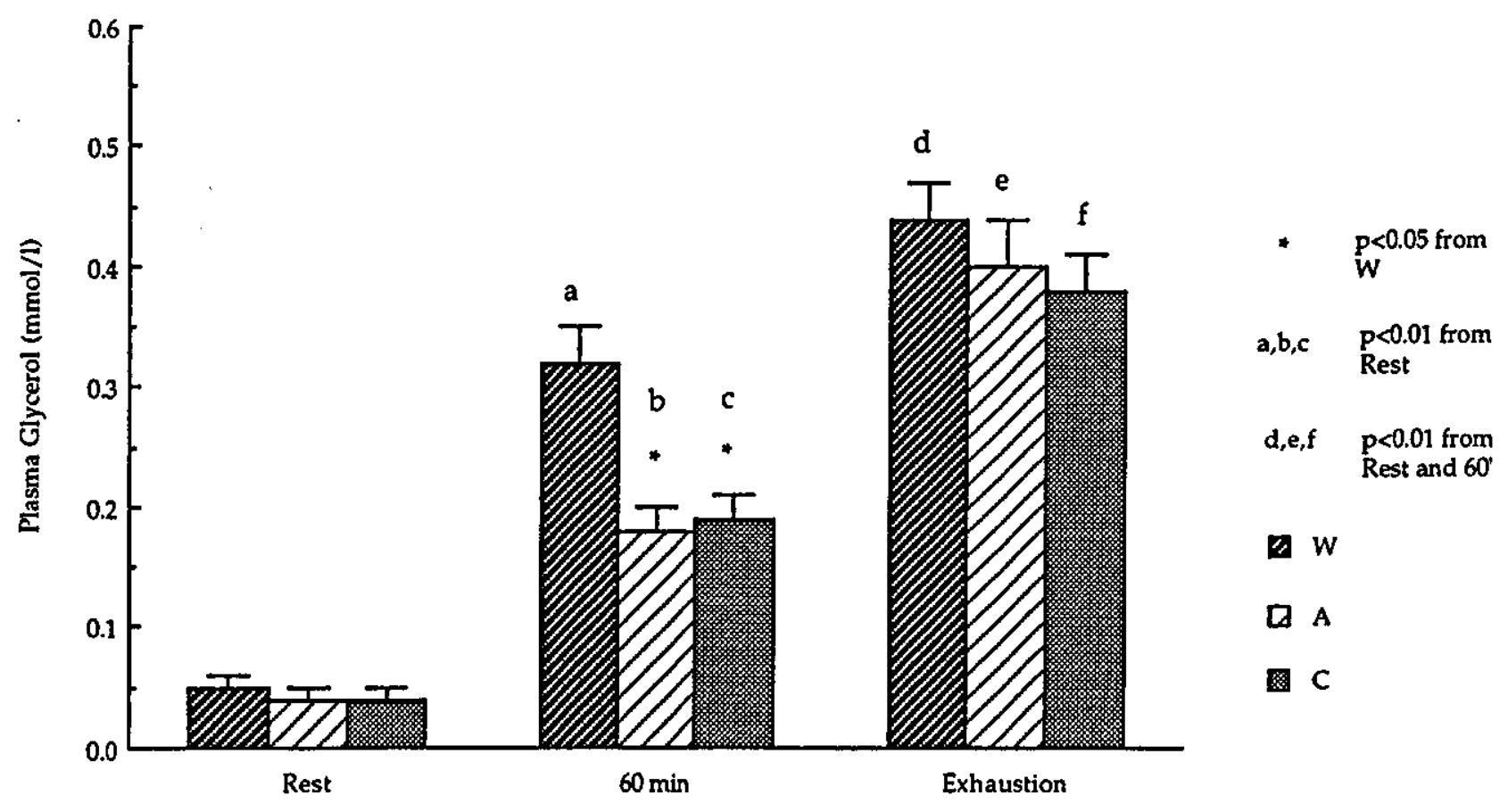

Fig. 5.6: Plasma glycerol concentrations (mmol/1) during the $W, A$ and $C$ trials (mean $\pm S E M, n=11$ ) 


\subsection{DISCUSSION}

The main finding of this study was a $14 \%$ improvement in endurance capacity as a result of ingesting a $5.5 \% \mathrm{CHO}$ solution during the first hour of prolonged running. A $6.9 \% \mathrm{CHO}$ solution, however, failed to improve endurance capacity compared with water. These results are in agreement with the previous study (see Chapter 4). In that study, an improved performance time over the marathon distance $(42.2 \mathrm{~km})$ was observed as a result of ingesting the same $5.5 \% \mathrm{CHO}$ solution compared with water ingestion. A $6.9 \% \mathrm{CHO}$ solution again did not show any significant difference from water.

Most of the studies in the literature have shown an improved endurance capacity for cycling exercise as a result of carbohydrate ingestion (Coyle et al. 1983; Coyle et al. 1986; Bjorkman et al. 1984; Maughan et al. 1989; Coggan and Coyle, 1989; Wright et al. 1991). In these studies a variety of $\mathrm{CHO}$ solutions between 4 and $50 \%$ have been used which resulted in a total $\mathrm{CHO}$ intake in the range of $36-406 \mathrm{~g}$. The improvement in endurance capacity ranged from $17 \%$ to $33 \%$.

On the other hand, as far as running exercise is concerned, conflicting results have been reported. Fruth and Gisolfi (1983) have shown no difference in running time to exhaustion at $70 \% \mathrm{VO}_{2} \max$ when placebo, glucose or fructose were ingested throughout the run. However, there was a tendency for a lower running time in the fructose trial compared with the other two trials. It should be pointed out that the small number of subjects $(n=5)$ and the relatively large standard error of performance times might have masked any beneficial effect of glucose on time to exhaustion. Riley et al. (1988) also failed to demonstrate an improved time to exhaustion in their subjects who ran at $70 \% \mathrm{VO}_{2}$ max following a 21 -h fast, when a $7 \% \mathrm{CHO}$ solution was ingested $20 \mathrm{~min}$ before and during the run as opposed to placebo intake. In that study the pre-exercise $\mathrm{CHO}$ feeding resulted in different plasma glucose and insulin concentrations at the start of exercise. It is possible that this different response coupled with the 21-hour fast might have altered the endurance capacity when $\mathrm{CHO}$ was ingested during the run. In contrast to the above results, Sasaki et al. (1987a) and Wilber and Moffat (1992) have observed an improved time to exhaustion when their subjects ran at $80 \%$ $\mathrm{VO}_{2}$ max as a result of ingesting $\mathrm{CHO}$ solutions compared with placebo (48\% 
and $29 \%$ improvement respectively). Furthermore, when a $5 \% \mathrm{CHO}$ solution was given during a run to exhaustion at $85 \%$ of maximum heart rate a $31 \%$ improvement was also reported compared with water ingestion (Macaraeg, 1983).

The improvement in endurance capacity in the present study averaged $14 \%$. This value is lower than those reported in other studies; it could be explained, however, by the low total $\mathrm{CHO}$ ingestion which averaged only 45 $\mathrm{g}$ and the restriction of the $\mathrm{CHO}$ ingestion to the early part of exercise. Additional benefits in the performance might have been gained if $\mathrm{CHO}$ had been ingested throughout the duration of exercise; a drinking pattern employed by most of the studies in the literature. In one study, however, $\mathrm{CHO}$ was ingested approximately $30 \mathrm{~min}$ prior to the point of fatigue in an attempt to reverse the decline in blood glucose concentration and $\mathrm{CHO}$ oxidation rate usually observed towards the end of prolonged cycling exercise. As a result a $21 \%$ improvement in endurance capacity was observed compared with placebo (Coggan and Coyle, 1989). It seems, however, that oral $\mathrm{CHO}$ supplementation at the actual point of fatigue would be of little benefit in preventing a decline in blood glucose concentration and delaying the onset of fatigue (Coggan and Coyle, 1987).

At this point it should be reminded that the ingestion of the $6.9 \% \mathrm{CHO}$ solution, which resulted in greater total $\mathrm{CHO}$ intake, did not significantly improve the time to exhaustion. This finding is in agreement with the previous study (see Chapter 4) but not with studies from other laboratories. Indeed, a 7\% CHO solution has been shown to delay the onset of fatigue during prolonged running (Wilber and Moffat, 1992; Millard-Stafford et al. 1992). Therefore, the critical difference between the two drinks used in the present study might not be the small difference in $\mathrm{CHO}$ concentration, but rather the effect of $\mathrm{CHO}$ composition on gastric emptying rate, intestinal absorption and $\mathrm{CHO}$ availability to the working muscles.

The initial high large bolus ( $8 \mathrm{ml} \cdot \mathrm{kg}^{-1}$ body weight) and the subsequent repeated drinking of small volumes $\left(2 \mathrm{ml} . \mathrm{kg}^{-1}\right)$ were designed in order to promote a rapid gastric emptying rate of the ingested solution (Rehrer et al. (1990). It has been suggested that gastric volume is a very important determinant of the rate of gastric emptying of fluids (Noakes et al. 1991b). Indeed, in the study by Rehrer et al. (1990) it was shown that almost all of a 
7.2\% CHO solution emptied the stomach within the first 60 min of exercise;

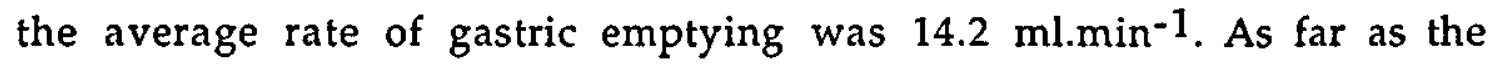
solutions used in the present study are concerned, no difference in gastric emptying rate was observed between the $\mathrm{A}$ and the $\mathrm{C}$ solutions (Maughan, personal communication). In agreement with the data from Rehrer et al. (1990), it was found that almost $95 \%$ of both solutions $\left(8 \mathrm{ml} . \mathrm{kg}^{-1}\right.$ body weight) emptied the stomach within the first $60 \mathrm{~min}$. In contrast, equivalent amount of water emptied the stomach within the first $30 \mathrm{~min}$. Thus, a difference in the gastric emptying rate between the two $\mathrm{CHO}$ solutions used in the present study can not explain the failure of the $C$ solution to significantly improve endurance capacity compared with the water trial.

The rate of intestinal absorption of the $\mathrm{CHO}$ solutions was not measured in this study. However, it is of interest to note that seven cases of gastrointestinal discomfort were recorded when the $6.9 \% \mathrm{CHO}$ solution was ingested; only three cases of such discomfort were observed when either water or the $5.5 \%$ solution were ingested. It is possible that gastrointestinal discomfort was associated with a reduced intestinal absorption of the $6.9 \%$ CHO solution (Brouns, 1991). The lower blood glucose response to the $6.9 \%$ solution compared with the $5.5 \%$ solution further supports such a link. On the other hand, an argument against such a hypothesis is the observation of higher $\mathrm{CHO}$ oxidation rates during the first $80 \mathrm{~min}$ of exercise when the $6.9 \%$ solution was ingested compared with water and the $5.5 \% \mathrm{CHO}$ solution. Nevertheless, the ingestion of the $6.9 \% \mathrm{CHO}$ was associated with higher occurrence of gastrointestinal discomfort, a factor which might have been responsible for the failure of this solution to significantly improve endurance capacity.

The results from this study demonstrate that during running exercise blood glucose concentration does not appear to decrease prior to the point of fatigue. Furthermore, $\mathrm{CHO}$ oxidation rates are similar when fed water or $\mathrm{CHO}$. During exercise without $\mathrm{CHO}$ ingestion serum catecholamines, cortisol and Growth Hormone $(\mathrm{GH})$ increased with time in spite of an unchanged blood glucose concentration. This supports the suggestion that blood glucose concentration is not the sole determinant of hormonal response to prolonged exercise (Galbo et al. 1979). Carbohydrate ingestion or infusion during prolonged exercise has been shown to suppress the increase in cortisol secretion usually observed during exercise without $\mathrm{CHO}$ ingestion or 
infusion (Tabata et al. 1991; Deuster et al. 1992). In the present study, $\mathrm{CHO}$ ingestion did not alter the plasma cortisol and catecholamine response to exercise in the presence of either unchanged (trial C) or elevated (trial A) blood glucose concentration. It has to be pointed out, however, that blood samples for hormonal determination were obtained only at rest, $60 \mathrm{~min}$ and exhaustion. Thus, a different hormonal response to the $\mathrm{CHO}$ feeding might have occurred earlier in exercise.

In contrast, $\mathrm{CHO}$ ingestion resulted in the suppression of $\mathrm{GH}$ concentration at $60 \mathrm{~min}$ of exercise. Plasma FFA and glycerol concentrations were also suppressed during the first hour of exercise. An interesting finding of this study, however, is that plasma FFA and glycerol and serum GH suppression was reversed as soon as $\mathrm{CHO}$ feeding was replaced with water ingestion from 60 min until exhaustion during both the $\mathrm{CHO}$ trials. At the point of exhaustion no difference in these metabolites was observed between the three trials. Since the changes in GH response paralleled those in FFA and glycerol, it seems that during prolonged exercise secretion of GH is very important for fat mobilization from adipose tissue. Furthermore, the reduced plasma FFA concentration in the $\mathrm{CHO}$ trials might have suppressed hepatic glucose production by reducing hepatic glycogenolysis (Felber et al. 1993). Indeed, $\mathrm{CHO}$ ingestion during exercise might result in reduced hepatic glycogenolysis and gluconeogenesis (Coggan and Coyle, 1991). Moreover, CHO supplementation in moderate amounts might reduce hepatic glucose production without any detectable changes in mobilization of metabolic substrates (Vissing et al. 1988). Although serum glucagon concentration was not determined in this study, the lower catecholamine to insulin ratio observed at $60 \mathrm{~min}$ of exercise in the $\mathrm{CHO}$ trials compared with $\mathrm{W}$ would support such a suggestion.

It should be pointed out that in most of the cycling studies, blood glucose concentration and the rate of total $\mathrm{CHO}$ oxidation gradually decrease during prolonged exercise performed without $\mathrm{CHO}$ ingestion, whereas they are maintained at higher levels when $\mathrm{CHO}$ is ingested during exercise (Coyle et al. 1986). Thus, according to Coyle and his colleagues, $\mathrm{CHO}$ ingestion during prolonged exercise improves performance primarily by maintaining blood glucose oxidation at sufficiently high rates late in exercise rather than by sparing the limited muscle glycogen in the active muscles. 
As mentioned previously, the results from this study show that during running exercise blood glucose concentration and $\mathrm{CHO}$ oxidation rate do not appear to decrease prior to the point of fatigue. Furthermore, despite the reversal of metabolic responses after $60 \mathrm{~min}$ in the $\mathrm{CHO}$ trials and the resulting similarity in these responses to the three trials at the point of exhaustion, the ingestion of the $5.5 \%$ CHO solution during the first hour of exercise resulted in a delay of the onset of fatigue. It is possible that, unlike cycling, during running exercise sparing of glycogen in the active muscles could be the mechanism by which $\mathrm{CHO}$ ingestion exerts its ergogenic effect.

It is also possible that a greater degree of dehydration and/or hyperthermia might explain the earlier onset of fatigue in the water trial. Changes in plasma volume, body weight loss, skin and core temperatures, and plasma electrolyte concentrations were similar in all three trials of this study. This is despite the shorter exercising time when water was ingested. Thus, it appears that the rate of dehydration and/or hyperthermia might have been faster in the water trial. Stated differently, the rate of rehydration when ingesting the $5.5 \%$ CHO solution might have been faster compared with plain water ingestion. This is despite the faster gastric emptying rate of water compared with both the $\mathrm{CHO}$ solutions. This further supports the notion that gastric emptying rate is not the sole determinant of the actual rate at which any solution enters the circulation. The rate of intestinal absorption has to be considered when studying the effectiveness of a given solution on rehydration during exercise. Indeed, a $6 \% \mathrm{CHO}$ solution has been shown to produce a faster fluid absorption from the small intestine compared with water (Gisolfi et al. 1990).

In summary, the results of this study showed that the ingestion of a 5.5\% $\mathrm{CHO}$ solution, unlike a $6.9 \%$ solution, during the first hour of exercise resulted in an improved time to exhaustion compared with water ingestion. It seems that sparing of muscle glycogen, rather than maintenance of blood glucose and $\mathrm{CHO}$ oxidation rate toward the latter stages of exercise, might have been responsible for this improvement in running endurance capacity. In spite of similar gastric emptying rates between the two $\mathrm{CHO}$ solutions, the ingestion of the $6.9 \% \mathrm{CHO}$ solution was associated with higher occurrence of gastrointestinal discomfort, a factor which might have been responsible for the failure of this solution to significantly improve endurance capacity. 


\section{CHAPTER 6}

\section{EFFECT OF CARBOHYDRATE INGESTION ON MUSCLE GLYCOGEN UTILIZATION IN TYPE I AND TYPE II FIBRES}

\subsection{INTRODUCTION}

There is some evidence that carbohydrate ingestion during prolonged exercise delays the onset of fatigue by decreasing the rate of muscle glycogen utilization (Bjorkman et al. 1984; Erickson et al. 1987). On the other hand, other investigators have shown that carbohydrate ingestion exerts its ergogenic effect by simply preventing the decline in blood glucose concentration and carbohydrate oxidation rate late in exercise, rather than sparing muscle glycogen (Coyle et al. 1986). All of these studies have used cycling as the mode of exercise. However, there is a lack of information relating to running exercise. Furthermore, little is known about the effect of carbohydrate ingestion on muscle glycogen utilization in different fibre types.

It is possible that during running exercise sparing of muscle glycogen rather than maintenance of blood glucose at euglycaemic levels is responsible for an improvement in endurance capacity. This hypothesis is based on observations from previous studies (see Chapters 4 and 5) that during running exercise without $\mathrm{CHO}$ ingestion blood glucose concentration and carbohydrate oxidation rate do not decline prior to fatigue. Moreover, carbohydrate oxidation rates do not seem to be affected by carbohydrate ingestion. Yet, endurance capacity was improved when $\mathrm{CHO}$ was ingested during the early part of exercise (see Chapter 5).

The aim of this study, therefore, was to examine whether or not carbohydrate supplementation during treadmill running at constant speed influences muscle glycogen utilization in Type I (slow twitch) and Type II (fast twitch) fibres. 


\subsection{METHODS}

Subjects: Seven, male, recreational runners participated in this study. The mean age, height, weight, $\mathrm{VO}_{2} \max$ and $\mathrm{HR} \max$ of the subjects were 29.2 $( \pm 2.1)$ years $( \pm S E M), 176.3( \pm 1.8) \mathrm{cm}, 75.2( \pm 4.1) \mathrm{kg}, 54.5( \pm 2.0) \mathrm{ml} . \mathrm{kg}^{-1} \cdot \mathrm{min}^{-}$ 1 and $190.0( \pm 2.2)$ bt. $\mathrm{min}^{-1}$, respectively.

Preliminary measurements: Following familiarization with treadmill running and experimental procedures, the subjects undertook two preliminary tests in order to determine: (i) the relationship between running speed and oxygen uptake, and (ii) the maximum oxygen uptake ( $\mathrm{VO}_{2} \mathrm{max}$ ). The protocols of these tests have been described elsewhere (see Chapter 3). One week before the first experimental trial, the subjects undertook a 60 -min treadmill run at $70 \% \mathrm{VO}_{2}$ max in order to fully familiarize themselves with the drinking pattern and the measurements used during the experimental trials.

Protocol of the study: The subjects completed two $60-\mathrm{min}$ runs at $70 \% \mathrm{VO}_{2}$ max on a motorized treadmill seven days apart (Fig. 6.1). On each occasion the subjects consumed either an isotonic, 5.5\% carbohydrate-electrolyte solution (CHO) or water $(\mathrm{W})$ throughout the run. The order of testing was randomly assigned. The $5.5 \%$ CHO solution was chosen because in our previous studies the ingestion of the same solution, unlike water, enhanced both endurance performance over a $42.2 \mathrm{~km}$ treadmill time trial (Chapter 4) and endurance capacity during a run to exhaustion at $70 \% \mathrm{VO}_{2}$ $\max$ (Chapter 5).

Both experimental trials took place following a 3-day standardised normal diet and a 12-14 hour overnight fast. A warm-up which consisted of 5-min run at $60 \% \mathrm{VO}_{2}$ max preceded each trial. The treadmill was then stopped for $5 \mathrm{~min}$ and the subject consumed $8 \mathrm{ml}^{\mathrm{kg}} \mathrm{kg}^{-1}$ body weight (bw) of the assigned fluid while standing on the treadmill. A further $2 \mathrm{ml} . \mathrm{kg}^{-1}$ of the assigned fluid was ingested by the subjects at 20 and $40 \mathrm{~min}$ of the run. Immediately after this 5 min period, the treadmill running speed was increased to $70 \% \mathrm{VO}_{2}$ max. Muscle biopsy samples were obtained at rest and immediately after the completion of the run. Blood samples were taken at rest, $10 \mathrm{~min}, 20 \mathrm{~min}, 40 \mathrm{~min}$ and at the end of the run. One minute expired air samples were collected at $10 \mathrm{~min}, 20 \mathrm{~min}, 40 \mathrm{~min}$ and during 
the last min of the run. The Borg scale of perceived exertion (Borg, 1973) and a simple 1-10 scale of perceived thirst were also used at the same intervals. Following expired air and blood sampling, the assigned fluid was ingested by the subjects at 20 and $40 \mathrm{~min}$ of the run. Wet sponges were available to the subjects to be used ad libitum throughout the runs. Heart rate was recorded continuously during the run. The subject's pre-exercise and post-exercise nude body weight was also obtained.

Dry bulb temperatures within the laboratory were $22.9 \pm 0.4{ }^{\circ} \mathrm{C}$ and $22.7 \pm$ $0.4^{\circ} \mathrm{C}$ during the $\mathrm{W}$ and $\mathrm{CHO}$ trials respectively and relative humidity for the two conditions were $50.0 \pm 1.0 \%$ and $51.3 \pm 2.1 \%$ respectively.

Analysis: Expired air samples were collected and analysed as previously described (see Chapter 3). Venous blood samples were analysed for blood lactate, glucose and haemoglobin concentrations and haematocrit values; plasma. FFA and glycerol; and serum insulin. Percentage changes in plasma volume were measured as previously described (see Chapter 3).

Muscle biopsy samples were quickly removed from the needle and immediately immersed in liquid nitrogen until they were freeze dried. Each sample was then divided into two parts before being stored at $-70 \mathrm{o}$. At a later date, one part of the freeze-dried muscle was dissected free of visible blood and connective tissue, powdered and washed twice with petroleum ether to remove the fat. Muscle metabolites (Glycogen, Glucose, G-6-P, Lactate, ATP, PCr and $\mathrm{Cr}$ ) were extracted and determined enzymatically as previously described (see Chapter 3).

The second part of the freeze-dried muscle, which was also washed twice with petroleum ether to remove the fat, was used to determine the glycogen content of single muscle fibres as previously described (see Chapter 3). Single fibre analysis was performed on the biopsy samples of six subjects.

Statistical analysis: A two-way analysis of variance (ANOVA) for repeated measures on two factors (experimental treatment and samplimg time) was used to compare differences between cardiovascular changes, blood metabolites and muscle metabolites for both trials. Body weight changes, plasma volume changes and volumes of fluid ingested during the trials 
were analysed by students $t$-test for correlated data. When using ANOVA any significant differences shown were assessed by applying the Tukey posthoc test. Statistical significance was accepted at a 5\% level. Results are presented as mean \pm SEM. 
Fluid ingestions
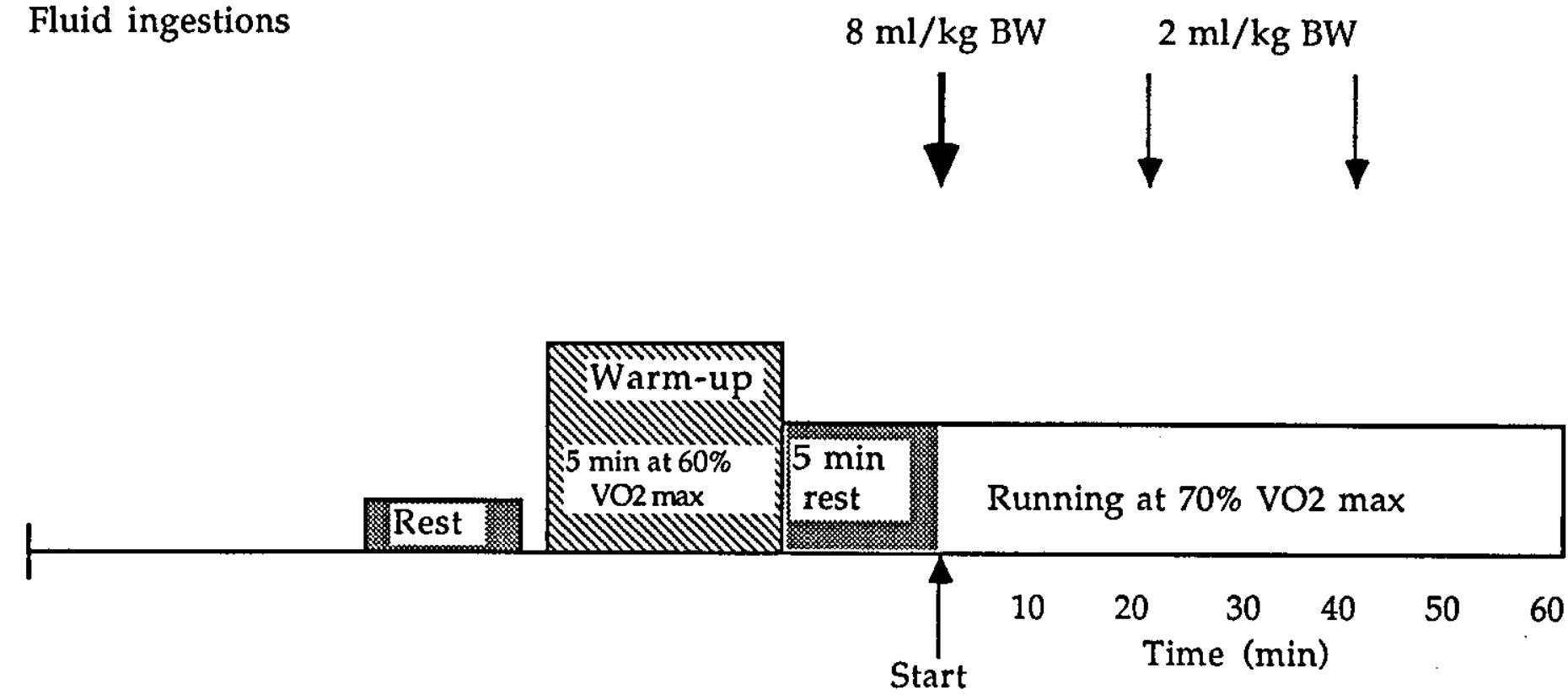

Venous blood samples
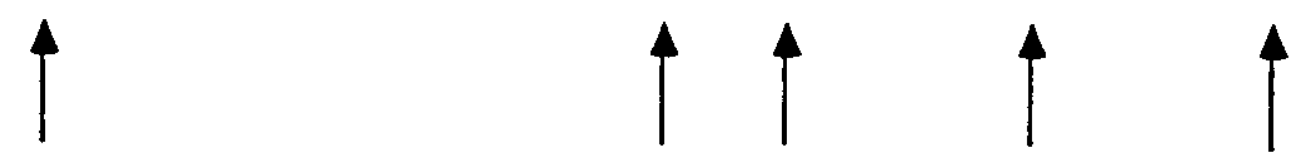

Muscle samples

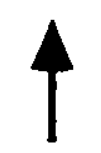

4

Expired air samples
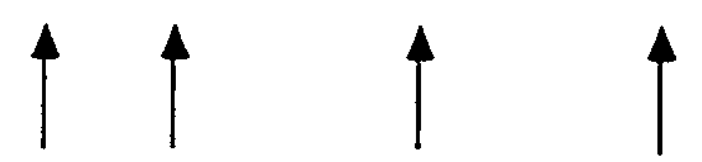

Fig. 6.1: Schematic illustration of the study's protocol 


\subsection{RESULTS}

The average $\% \mathrm{VO}_{2}$ max sustained during the $\mathrm{W}$ and $\mathrm{CHO}$ trials was $73.1 \pm$ $1.0 \%$ and $71.9 \pm 1.7 \%$, respectively. There was no difference in $\% \mathrm{VO}_{2} \max$ between trials (Table 6.1). Respiratory Exchange ratio (RER) were also similar between trials (Table 1). Carbohydrate oxidation rate, as calculated from $\mathrm{VO}_{2}$ and RER values, averaged $2.44 \pm 0.30 \mathrm{~g} \cdot \mathrm{min}^{-1}$ and $2.51 \pm 0.27$ g. min $^{-1}$ during the $\mathrm{W}$ and $\mathrm{CHO}$ trials, respectively. No difference was found between trials (Table 6.1). Carbohydrate ingestion did not alter the heart rate response to exercise. In both trials, however, heart rate was higher at the end of the run as compared with the 10 min point $(p<0.01)$ (Table 6.1).

The subjects ingested a total of $894 \pm 50 \mathrm{ml}$ and $896 \pm 49 \mathrm{ml}$ of fluid during the $\mathrm{W}$ and $\mathrm{CHO}$ trials, respectively. In the latter trial, this fluid ingestion resulted in the consumption of $49.3 \pm 2.7 \mathrm{~g}$ of $\mathrm{CHO}$. In both trials, $60 \mathrm{~min}$ of running induced a body weight loss of $1.5 \pm 0.2 \mathrm{~kg}$ or $2.1 \pm 0.2 \%$. Body weight loss was corrected for fluid intake. Percent change of plasma volume was also similar between the two trials. A reduction of $7.3 \pm 1.6 \%$ and $6.2 \pm 1.5 \%$ was observed in the $\mathrm{W}$ and $\mathrm{CHO}$ trials, respectively. Perceived rate of exertion ranged from 10 to 13 on the Borg scale, and there were no differences between trials. Similarly, no difference was found between the trials for perceived rate of thirst.

Blood glucose concentration was higher $(p<0.01)$ throughout the run during the $\mathrm{CHO}$ trial compared with the $\mathrm{W}$ trial (Fig. 6.2), whereas serum insulin concentration (Fig. 6.3) was only higher at $20 \mathrm{~min}$ of exercise (10.5 \pm $1.6 \mathrm{mU.1} \mathrm{I}^{-1}$ vs $\left.5.2 \pm 0.3 \mathrm{mU.1} \mathrm{l}^{-1}, \mathrm{p}<0.01\right)$. On the other hand, no difference was observed between trials in blood lactate concentration (Fig. 6.4). Carbohydrate ingestion resulted in lower plasma FFA (Fig. 6.5) and glycerol (Fig. 6.6) concentrations throughout the run compared with water $(p<$ 0.01 ). Water ingestion produced a steady increase in the concentrations of both metabolites. On the other hand, at $20 \mathrm{~min}$ of exercise in the CHO trial plasma FFA concentration declined below resting values $(p<0.05)$. At the end of the run, however, the plasma FFA values returned to those recorded before exercise. 
Table 6.2 shows that glycogen utilization was lower in the CHO trial compared with W $\left(108.7 \pm 16.3 \mathrm{mmol}^{\mathrm{kg} \mathrm{dm}}{ }^{-1}\right.$ vs $150.9 \pm 19.9 \mathrm{mmol}^{\mathrm{kg} \mathrm{dm}}{ }^{-}$ 1 respectively, $\mathrm{p}<0.01)$. The average rates of glycogen utilization during the $\mathrm{CHO}$ and $\mathrm{W}$ trials were also different $\left(1.8 \pm 0.3 \mathrm{mmol} . \mathrm{kg} \mathrm{dm}^{-1} \cdot \mathrm{min}^{-1} \mathrm{vs}\right.$ $2.5 \pm 0.3 \mathrm{mmol} . \mathrm{kg} \mathrm{dm}^{-1} \cdot \mathrm{min}^{-1}$ respectively, $\mathrm{p}<0.01$ ). These results clearly demonstrate a $28 \%$ reduction in glycogen utilization as a result of $\mathrm{CHO}$ ingestion compared with water ingestion $(p<0.01)$. Carbohydrate ingestion also resulted in lower muscle G6P $\left(1.9 \pm 0.3 \mathrm{mmol}^{\mathrm{kg} \mathrm{dm}} \mathrm{m}^{-1}\right.$ vs $2.6 \pm 0.2$

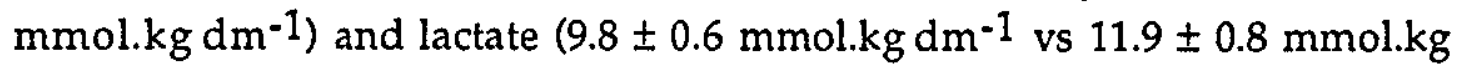
$\mathrm{dm}^{-1}$ ) concentrations at the end of the run compared with $W$ (Table 6.2).

Muscle glycogen concentrations (mmol glucose.kg dm-1 ) in Type I and Type II fibres are shown in Table 6.3. Muscle glycogen concentration at rest was higher $(p<0.01)$ in Type II fibres $\left(367.0 \pm 25.0 \mathrm{mmol}\right.$ glucose. $\mathrm{kg} \mathrm{dm}^{-1}$ in the $W$ trial and $377.5 \pm 30.2 \mathrm{mmol}$ glucose. $\mathrm{kg} \mathrm{dm}^{-1}$ in the CHO trial) when compared with Type I fibres $\left(301.9 \pm 30.2 \mathrm{mmol}\right.$ glucose. $\mathrm{kg} \mathrm{dm}^{-1}$ in the $\mathrm{W}$ trial and $305.0 \pm 34.0 \mathrm{mmol}$ glucose. $\mathrm{kg} \mathrm{dm}^{-1}$ in the $\mathrm{CHO}$ trial). Exercise without $\mathrm{CHO}$ ingestion resulted in a greater $(\mathrm{p}<0.01)$ degradation of muscle glycogen in Type I fibres $(66.2 \%$ of the resting value, $\mathrm{p}<0.01)$ compared with Type II fibres (20.0\% of the resting value, $p<0.01$ ). The ingestion of the $\mathrm{CHO}$ solution resulted in sparing of glycogen in Type I fibres (38.1\% degradation of glycogen as opposed to $66.2 \%$ during the $\mathrm{W}$ trial, $\mathrm{p}=0.01$ ). On the other hand, glycogen utilization was $17.5 \%$ in Type II fibres and it was not different from $W$.

In the $\mathrm{W}$ trial, the rate of glycogen utilization in Type I fibres averaged $3.3 \pm$ $0.3 \mathrm{mmol} . \mathrm{kg} \mathrm{dm}^{-1} \cdot \mathrm{min}^{-1}$ over the $60 \mathrm{~min}$ run. In Type II fibres the corresponding rate was almost three times lower averaging $1.2 \pm 0.3$ mmol.kg dm${ }^{-1} \cdot \mathrm{min}^{-1}(\mathrm{p}<0.01)$. The ingestion of $\mathrm{CHO}$ resulted in lower rate of glycogen utilization in Type I fibres compared with $W(1.9 \pm 0.4$ mmol. $\mathrm{kg} \mathrm{dm}^{-1} \cdot \mathrm{min}^{-1}$ vs $3.3 \pm 0.3 \mathrm{mmol} . \mathrm{kg} \mathrm{dm}^{-1} \cdot \mathrm{min}^{-1}$ respectively, $\mathrm{p}<$ 0.01 ). The rate of glycogen utilization in Type II fibres was, however, unaffected by $\mathrm{CHO}$ ingestion compared with water $\left(1.1 \pm 0.2 \mathrm{mmol} . \mathrm{kg} \mathrm{dm}{ }^{-}\right.$ 1 . $\mathrm{min}^{-1}$ vs $1.2 \pm 0.3 \mathrm{mmol} . \mathrm{kg} \mathrm{dm}^{-1} \cdot \mathrm{min}^{-1}$ respectively). In the $\mathrm{CHO}$ trial, the rate of glycogen utilization in Type I fibres was higher than in Type II fibres (1.9 $\pm 0.4 \mathrm{mmol} . \mathrm{kg} \mathrm{dm}^{-1} \cdot \mathrm{min}^{-1}$ vs $1.1 \pm 0.2 \mathrm{mmol} . \mathrm{kg} \mathrm{dm}^{-1} \cdot \mathrm{min}^{-1}$ respectively). This difference, however, failed to reach statistical significance $(p=0.06)$. 
Table 6.1: $\% \mathrm{VO}_{2}$ max, Respiratory Exchange Ratio (RER), $\mathrm{CHO}$ oxidation rate (CHO-OR) (g.min ${ }^{-1}$ ) and Heart Rate (HR) (bt.min ${ }^{-1}$ ) during the $W$ and CHO trials (mean $\pm S E M, n=7$ )

Variables

$\% \mathrm{VO}_{2} \max$

$\begin{array}{lllll}\text { W } & 71.3 & 71.7 & 73.9^{*} & 75.4^{* *} \\ \text { SEM } & \pm 1.0 & \pm 1.0 & \pm 1.0 & \pm 1.0 \\ \text { C HO } & 71.4 & 70.7 & 72.1 & 73.4 \\ \text { SEM } & \pm 1.8 & \pm 1.4 & \pm 1.7 & \pm 2.0\end{array}$

RER

$\begin{array}{lrrrr}\text { W } & 0.94 & 0.90 & 0.88 & 0.89 \\ \text { SEM } & \pm 0.03 & \pm 0.01 & \pm 0.02 & \pm 0.02 \\ \text { C HO } & 0.92 & 0.91 & 0.91 & 0.94 \\ \text { SEM } & \pm 0.02 & \pm 0.02 & \pm 0.02 & \pm 0.03\end{array}$

CHO-OR

$\begin{array}{lrrrr}\text { W } & 2.75 & 2.34 & 2.25 & 2.43 \\ \text { SEM } & \pm 0.38 & \pm 0.21 & \pm 0.28 & \pm 0.34 \\ \text { CHO } & 2.55 & 2.42 & 2.37 & 2.70 \\ \text { SEM } & \pm 0.23 & \pm 0.22 & \pm 0.23 & \pm 0.38\end{array}$

HR

$$
\begin{aligned}
& W \\
& \text { SEM } \\
& \text { CHO } \\
& \text { SEM } \\
& \\
& \text { min }
\end{aligned}
$$

* $p<0.01$ from $10 \mathrm{~min}$

$\underline{10-\min } \quad \underline{20-\mathrm{min}} \quad \underline{40-\mathrm{min}} \quad \underline{60-\mathrm{min}}$ 
Table 6.2 : Muscle metabolites (mmol.kg dm-1) in m.quadriceps femoris before and after the $60-\mathrm{min}$ run in the $\mathrm{W}$ and CHO trials (mean $\pm S E M, n=7$ )

\begin{tabular}{|c|c|c|c|c|}
\hline & \multicolumn{2}{|c|}{$W$} & \multicolumn{2}{|c|}{$\mathrm{CHO}$} \\
\hline & PRE & POST & PRE & POST \\
\hline Glycogen & $342.3 \pm 21.0$ & $191.4 \pm 28.7^{* *}$ & $343.7 \pm 27.2$ & $235.0 \pm 33.1$ \\
\hline Glucose & $1.9 \pm 0.2$ & $3.3 \pm 0.5$ & $2.3 \pm 0.2$ & $4.3 \pm 0.3$ \\
\hline G6P & $1.3 \pm 0.1$ & $2.6 \pm 0.2^{*}$ & $1.2 \pm 0.1$ & $1.9 \pm 0.3$ \\
\hline Lactate & $6.1 \pm 0.7$ & $11.9 \pm 0.8^{* *}$ & $5.9 \pm 0.5$ & $9.8 \pm 0.6$ \\
\hline ATP & $24.4 \pm 1.0$ & $22.4 \pm 0.5$ & $25.6 \pm 0.9$ & $22.5 \pm 0.7$ \\
\hline $\mathrm{PCr}$ & $79.6 \pm 4.6$ & $59.8 \pm 5.4$ & $76.1 \pm 4.4$ & $59.8 \pm 4.6$ \\
\hline
\end{tabular}

Significantly different from $\mathrm{CHO}:{ }^{*} \mathrm{p}<0.05{ }^{* *} \mathrm{p}<0.01$

Table 6.3: Muscle glycogen concentrations (mmol glucose.kg dm${ }^{-1}$ ) in Type I fibres and Type II fibres at rest and $60 \mathrm{~min}$ in the $\mathrm{W}$ and CHO trials (mean $\pm S E M, n=6$ ).

\begin{tabular}{|c|c|c|c|c|c|c|c|}
\hline \multicolumn{4}{|c|}{$w$} & \multicolumn{4}{|c|}{ CHO } \\
\hline \multicolumn{2}{|c|}{ PRE-EXERCISE } & \multicolumn{2}{|c|}{ POST-EXERCISE } & \multicolumn{2}{|c|}{ PRE-EXERCISE } & \multicolumn{2}{|c|}{ POST-EXERCISE } \\
\hline TYPE I & TYPE II & TYPE I & TYPE II & TYPE I & TYPE II & TYPE I & TYPE II \\
\hline 301.9 & $367.0^{\mathrm{b}}$ & 102.0 & 293.6 & 305.0 & $377.5^{\mathrm{b}}$ & $188.9^{a}$ & 311.6 \\
\hline \pm 30.2 & \pm 25.0 & \pm 18.5 & \pm 29.1 & \pm 34.0 & \pm 30.2 & \pm 33.6 & \pm 36.2 \\
\hline
\end{tabular}

$a=p=0.01$ from $W$

$\mathrm{b}=\mathrm{p}<0.01$ from Type I 


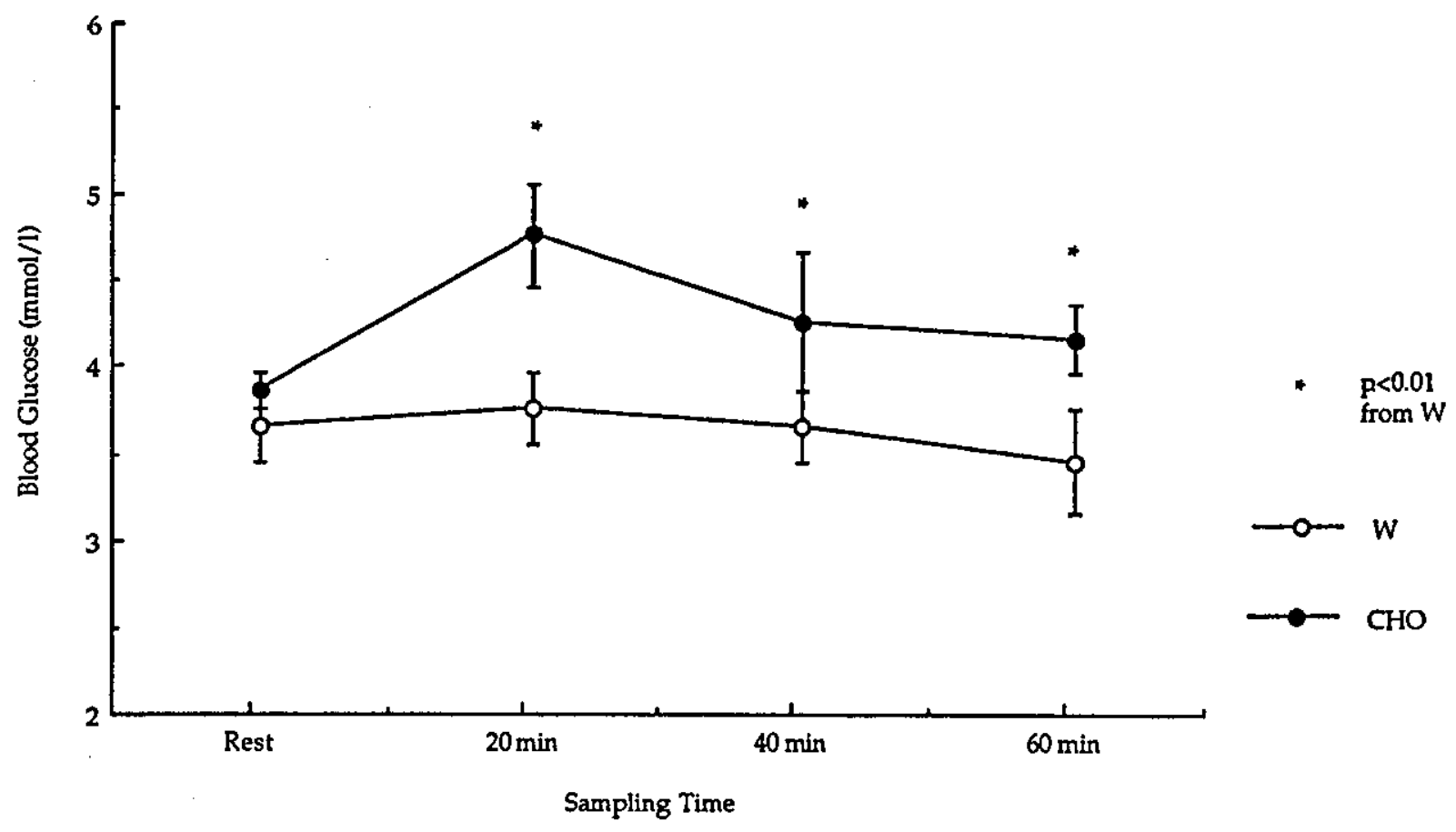

Fig. 6.2: Blood glucose concentrations (mmol/1) during the $\mathrm{W}$ and $\mathrm{CHO}$ trials (mean $\pm \mathrm{SEM}, \mathrm{n}=7$ )

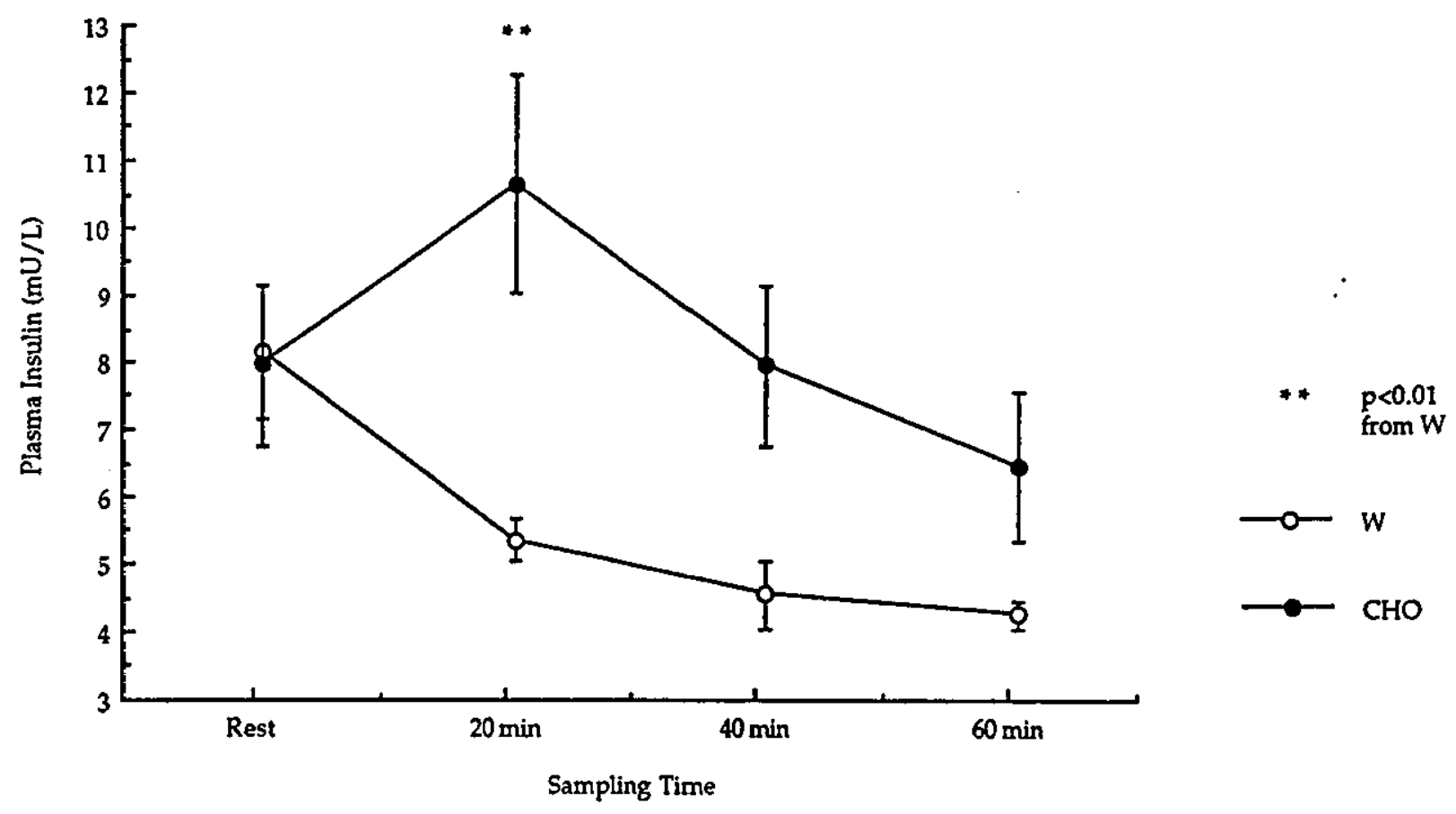

Fig. 6.3: Plasma insulin concentrations (mU/1) during the $\mathrm{W}$ and $\mathrm{CHO}$ trials (mean \pm SEM, $n=7$ ) 


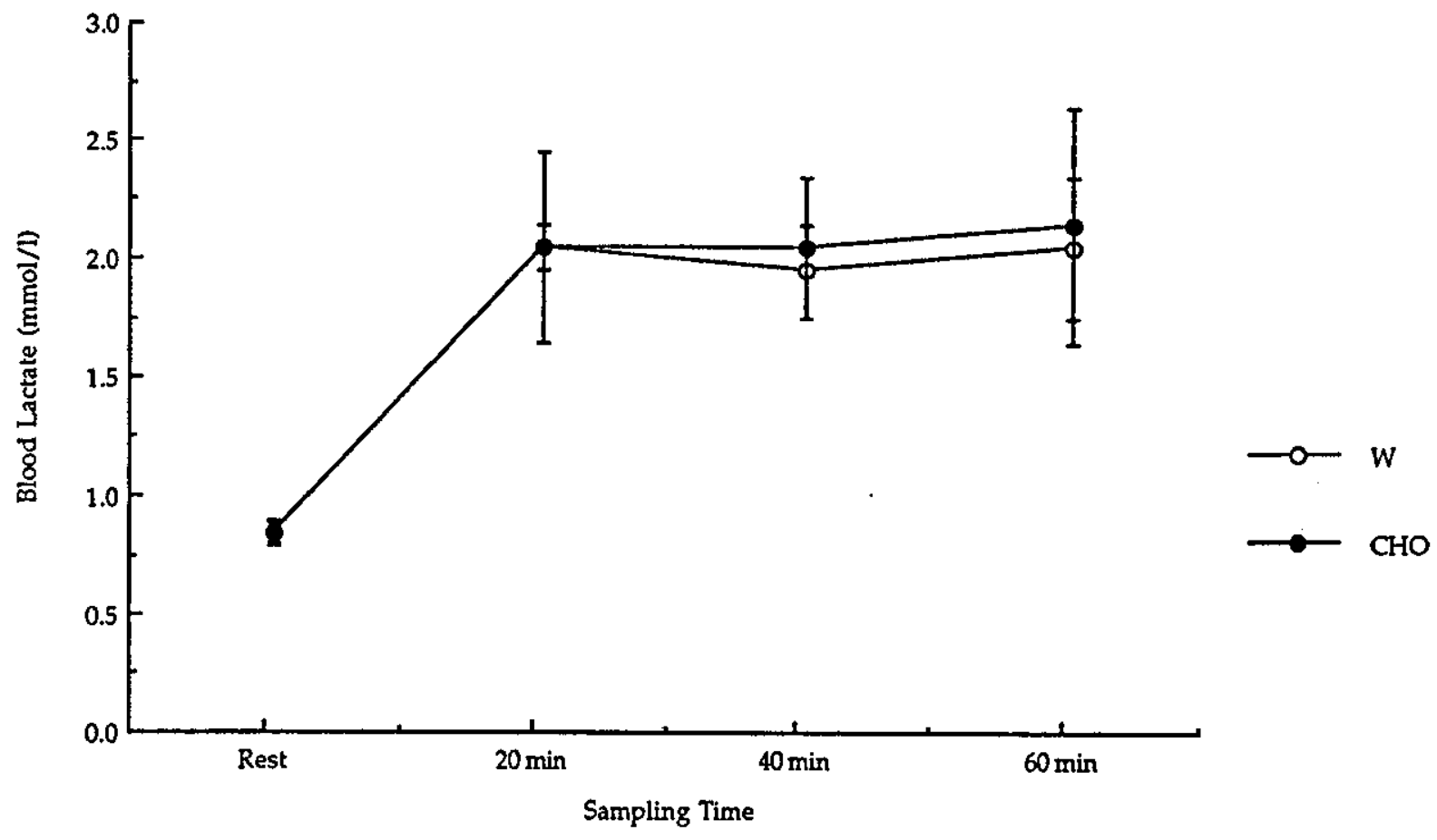

Fig. 6.4: Blood lactate concentrations (mmol/) during the $W$ and $C H O$ trials (mean $\pm S E M, n=7$ ) 


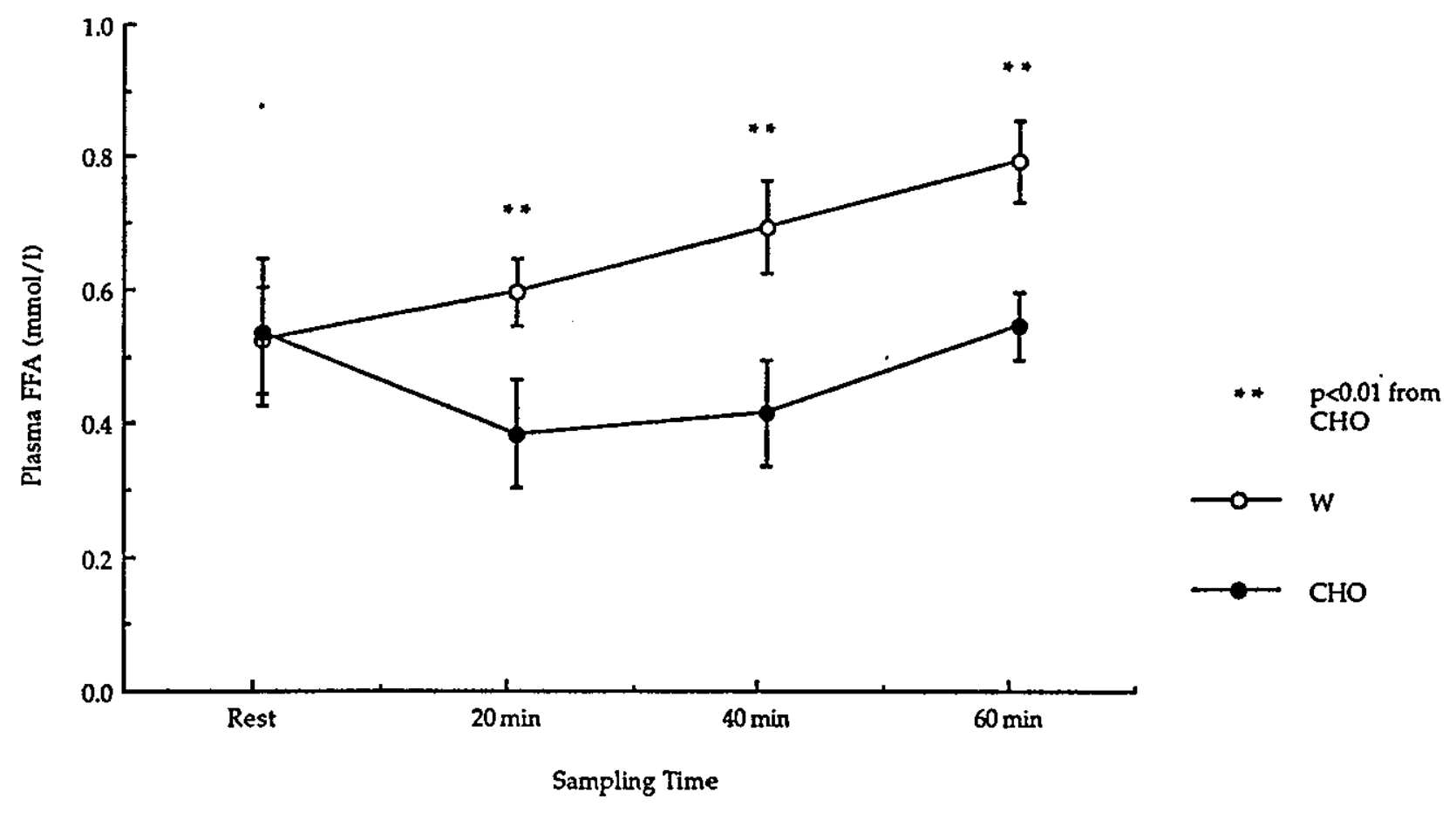

Fig. 6.5: Plasma FFA concentrations (mmol/l) during the $W$ and $C H O$ trials (mean $\pm S E M, n=7$ )

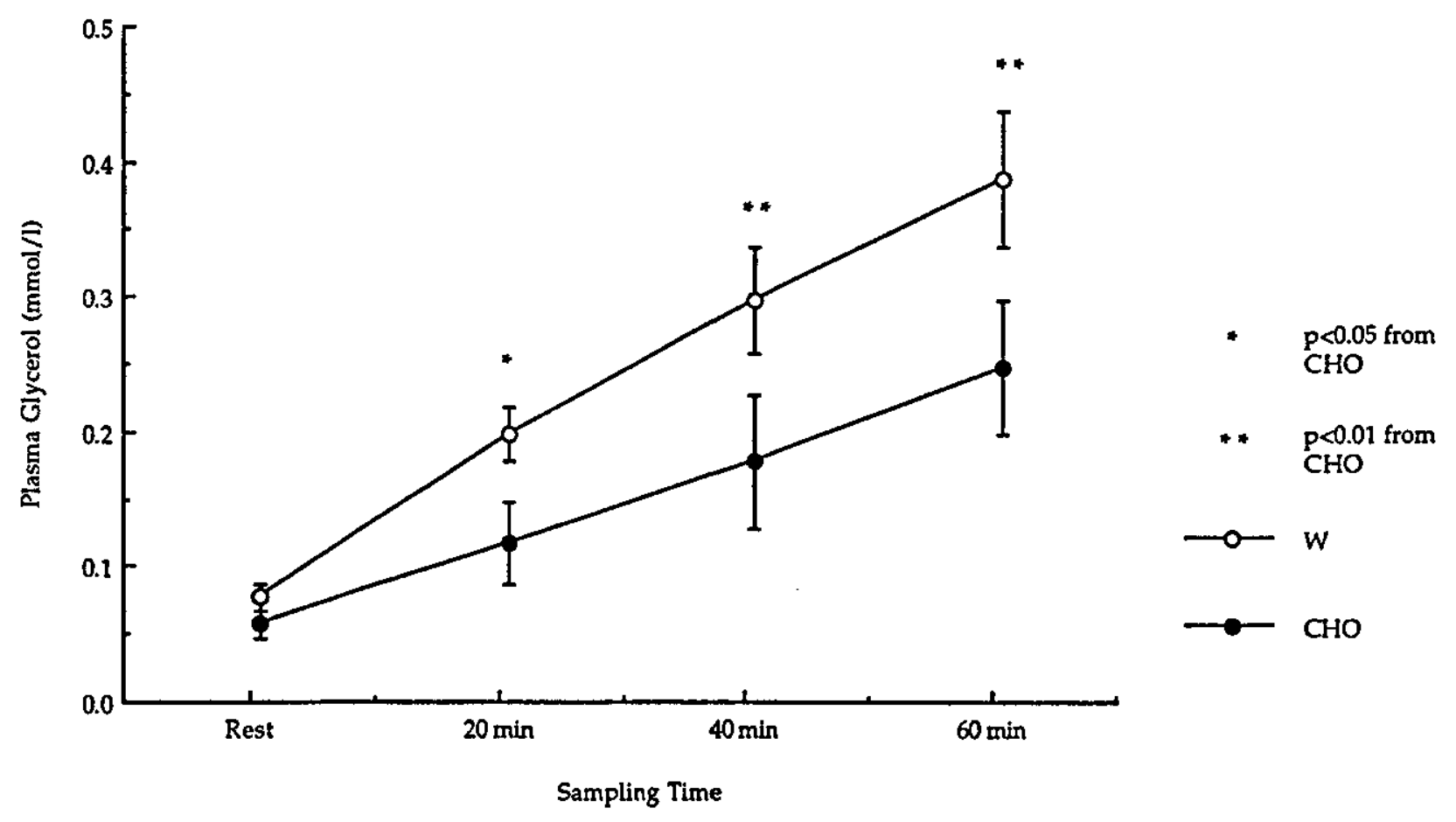

Fig. 6.6: Plasma glycerol concentrations (mmol//) during the $W$ and $\mathrm{CHO}$ trials (mean $\pm S E M, n=7$ ) 


\subsection{DISCUSSION}

The main finding of this study was that the ingestion of a $5.5 \% \mathrm{CHO}$ solution during a $60 \mathrm{~min}$ run resulted in a $28 \%$ sparing of muscle glycogen. Moreover, this reduced muscle glycogen utilization was confined mainly in Type I rather than Type II fibres.

The results of the present study are, therefore, in agreement with previous studies which have shown that both intravenous infusion of glucose (Bergstrom and Hultman, 1967) and oral ingestion of $\mathrm{CHO}$ solutions (Hargreaves et al. 1984; Bjorkman et al. 1984; Erickson et al. 1987) decrease the rate of glycogen utilization during submaximal exercise.

Other studies, however, have failed to demonstrate a glycogen sparing effect as a result of $\mathrm{CHO}$ ingestion when compared with water placebo (Coyle et al. 1986; Flynn et al. 1987; Hargreaves and Briggs, 1988; Mitchell et al. 1989a). In most of these studies, however, pre-exercise muscle glycogen concentrations were above normal and much higher compared with the present study. It is not known to what extent this factor might have contributed to the absence of glycogen sparing effect as a result of $\mathrm{CHO}$ ingestion. Furthermore, all of the above studies have used cycling as a mode of exercise. The existing evidence suggests that blood glucose concentration and the rate of total $\mathrm{CHO}$ oxidation gradually decrease during cycling exercise performed without $\mathrm{CHO}$ ingestion, whereas they are maintained at higher levels when $\mathrm{CHO}$ is ingested during exercise (Coyle et al. 1986). Thus, subjects are able to exercise longer when receiving $\mathrm{CHO}$ by simply being able to maintain blood glucose oxidation at sufficiently high rates late in exercise rather than by sparing the limited muscle glycogen in the active muscles (Coyle et al. 1986).

Observations from our laboratory, however, have shown that during constant pace running without $\mathrm{CHO}$ ingestion blood glucose concentration and carbohydrate oxidation rate do not decline prior to fatigue (Chapters 4 and 5). Moreover, the results from previous studies (Williams et al. 1990; Chapters 4 and 5) and the present study have shown that carbohydrate oxidation rates do not seem to be affected by carbohydrate ingestion. It follows, therefore, that $\mathrm{CHO}$ ingestion during running exercise would not 
affect endurance capacity by simply preventing the decline in blood glucose concentration and carbohydrate oxidation rate.

The results of this study suggest that during running exercise sparing of muscle glycogen could be the mechanism by which $\mathrm{CHO}$ ingestion exerts its ergogenic effect. The ingestion of almost any solution containing $\mathrm{CHO}$ would restore a declining blood glucose concentration and, perhaps, in this way maintain, and even increase, blood glucose oxidation during the latter stages of prolonged exercise. This is probably the case in cycling studies where a variety of $\mathrm{CHO}$ solutions of different compositions and concentrations were shown to improve endurance capacity (Coyle et al. 1983; Coyle et al. 1986; Bjorkman et al. 1984; Maughan et al. 1989; Coggan and Coyle, 1989; Wright et al. 1991). What is not known, however, is the exact mechanism by which a certain $\mathrm{CHO}$ solution could affect the rate of glycogen utilization. The question then rises as to whether or not any $\mathrm{CHO}$ solution could interfere with either glycogen breakdown or resynthesis. If not, this could explain the conflicting results obtained from studies investigating the effect of $\mathrm{CHO}$ ingestion on running endurance capacity (Macaraeg, 1983; Sasaki et al. 1987a; Wilber and Moffat, 1992; Riley et al. 1988; Fruth and Gisolfi, 1983).

The reduced breakdown of muscle glycogen in the $\mathrm{CHO}$ trial could theoretically be explained by a decreased activity of phosphorylase $\mathbf{a}$ the active form of the enzyme responsible for glycogen breakdown (Chasiotis et al. 1982). An altered adrenaline response is believed to affect glycogen breakdown (Richter et al. 1982) mainly through beta-adrenoceptors (Williams, 1986). There is evidence that beta-adrenoceptor density is twice as high in Type I fibres compared with Type II fibres (Martin et al. 1989). In a previous study of running at a constant pace using the same protocol and $\mathrm{CHO}$ solution, no difference was found in plasma adrenaline concentrations either before or at $60 \mathrm{~min}$ between the $\mathrm{CHO}$ and control trials (Study 2, Chapter 5). Unfortunately, plasma adrenaline response was not measured throughout the $60 \mathrm{~min}$ run. It is possible that this response might have been different in the first part of the $\mathrm{CHO}$ trial where the glycogenolytic rate is faster and most of the glycogen sparing might have occurred. However, it has been suggested that during submaximal exercise plasma adrenaline might not be as important a regulator of carbohydrate metabolism as it is of fat metabolism (Spriet et al. 1992). 
The activity of the enzyme phosphorylase was not measured in the present study. Prolonged exercise results in a rapid conversion of the a form of phosphorylase back to $\underline{b}$ form (Chasiotis et al. 1982). However, although reduced in concentration the activity of the a form is actually increased (Chasiotis, personal communication). The lower muscle G-6-P concentration observed at the end of the $\mathrm{CHO}$ trial compared with $\mathrm{W}$ might simply reflect a lower glycogenolytic rate which could have been brought about by a decreased activity of the $\underline{\mathbf{a}}$ form of the phosphorylase enzyme.

An increase in fat metabolism has a glycogen sparing effect (Costill et al. 1977; Hickson et al. 1977). An increased fat metabolism would result in inhibition of glycolysis at the PFK step, a subsequent accumulation of G-6-P, which could lead to inhibition of glycogenolysis by inhibiting phosphorylase $\mathfrak{b}$ activity (Aragon et al. 1980). However, the total fat oxidation rates, as calculated from $\mathrm{RER}$ and $\mathrm{VO}_{2}$ values, were similar in the two trials. This is despite the significant suppression of plasma FFA as a result of $\mathrm{CHO}$ ingestion. It seems that intramuscular triacylglycerol breakdown was increased in the latter trial to compensate for the reduced availability of plasma FFA (Romijin et al. 1993). Moreover, G-6-P concentration was lower and not higher at the end of the $\mathrm{CHO}$ trial compared with $W$. Thus, in the presence of similar contribution of fat metabolism to energy supply in the working muscles with and without carbohydrate ingestion, the exact mechanism by which $\mathrm{CHO}$ ingestion affects glycogen breakdown remains to be investigated.

The higher glycogen concentration found at rest in Type II fibres compared with Type I fibres is in agreement with a previous study (Greenhaff et al. 1991) and possibly reflects the higher glycogenolytic capacity of these fibres. The contribution, however, of these fibres towards energy supply to the working muscles at the exercise intensity employed in this study $\left(70 \% \mathrm{VO}_{2}\right.$ max) was small as reflected by the total amount of glycogen breakdown in these fibres. Furthermore, it is possible that this contribution was confined within the first 10-15 min of exercise, a period where glycogen breakdown and consequently lactate accumulation are very high (Spriet et al. 1992). Taking into account that glycogen concentration was not determined in the subgroups of Type II fibres, namely IIA, IIAB and IIB, it is also likely that most of the glycogen breakdown measured in Type II fibres actually 
occurred in Type IIA fibres. Indeed, it has been shown that most of Type IIA fibres are recruited right from the beginning of exercise and manifest a similar rate of glycogen utilization to Type I fibres at an exercise intensity which elicits about $75 \% \mathrm{VO}_{2} \max$ (Vollestad et al. 1984).

In the present study, an almost three times higher rate of glycogen utilization was observed in Type I fibres compared with Type II fibres when water was ingested. A similar finding was reported by Essen (1978) during cycling exercise of similar duration but lower intensity (50-60\% $\mathrm{VO}_{2}$ max). In that study, however, the actual rate of glycogen utilization in Type 1 fibres was higher compared with the present study $\left(4.6 \mathrm{mmol} . \mathrm{kg} \mathrm{dm}^{-1}\right.$. $\mathrm{min}^{-}$ 1 vs $3.3 \mathrm{mmol} . \mathrm{kg} \mathrm{dm}^{-1} \cdot \mathrm{min}^{-1}$ respectively). Considering the lower intensity in the study by Essen (1978), it could be argued that a heavier workload is probably imposed on the quadriceps muscle during cycling compared with running exercise. However, this by no means implies that vastus lateralis muscle is less involved than gastrocnemius muscle during running exercise. Indeed, in one study, biopsy samples were obtained from the gastrocnemius muscle before and after a 70 -min treadmill run at $78 \% \mathrm{VO}_{2}$ max (Madsen et al. 1990). Despite the higher initial muscle glycogen concentration in that study, the average rate of glycogen utilization in the mixed muscle was similar $\left(2.2 \mathrm{mmol} . \mathrm{kg} \mathrm{dm}^{-1} \cdot \mathrm{min}^{-1}\right)$ to the value observed in the present study $\left(2.5 \mathrm{mmol} . \mathrm{kg} \mathrm{dm}^{-1}\right.$. $\left.\mathrm{min}^{-1}\right)$ where biopsy samples were taken from the vastus lateralis muscle. This would suggest that, at least during treadmill running, vastus lateralis muscle is as heavily involved as the gastrocnemius muscle (see also Chapter 3 ).

In another study, a much higher rate of glycogen utilization (i.e. 8.6 mmol.kg dm${ }^{-1} \cdot \mathrm{min}^{-1}$ ) in Type I fibres was found after $60 \mathrm{~min}$ of cycling exercise at $61 \% \mathrm{VO}_{2} \max$ (Vollestad et al. 1984). This discrepancy in the results between the studies by Essen (1978) and Vollestad et al. (1984) could be explained by the different methodology employed in those studies. A direct biochemical analysis on single fibres was performed by Essen (1978), as in the present study. On the other hand, Vollestad et al. (1984) employed a histochemical method to quantify glycogen content in single fibres.

The high glycogen utilization in Type I fibres confirms the role of this population of fibres as the main contributor to locomotion during submaximal exercise. This is also evident by the fact that almost all of the 
glycogen sparing occurred in Type I fibres; Type II fibres were unaffected by $\mathrm{CHO}$ ingestion. A further implication of this, is that if glycogen sparing was brought about by enhanced glycogen resynthesis as opposed to decreased glycogen breakdown, one would expect glycogen sparing to be restricted to Type II fibres which are recruited to minor extent during $60 \mathrm{~min}$ of exercise at $70 \% \mathrm{VO}_{2}$ max. Thus, it could be argued that a lower glycogen breakdown and not increased glycogen resynthesis is responsible for the observed glycogen sparing in this study.

In the present study similar total $\mathrm{CHO}$ and fat oxidation rates with and without $\mathrm{CHO}$ feeding were observed. Under such conditions, a decreased glycogen utilization in Type I fibres would reflect a higher oxidation of blood glucose by those fibres as a result of $\mathrm{CHO}$ ingestion. Thus, it seems that skeletal muscle fibres are capable of increasing blood glucose uptake not only during the latter stages of prolonged exercise (Coyle et al. 1986) or when their muscle glycogen content is depleted (Gollnick et al. 1981), but also early in exercise in the presence of sufficient glycogen content. Although a suppressed plasma FFA concentration could favour an increased blood glucose uptake by the active muscles (Pernow and Saltin, 1971), the increased glucose uptake is probably insulin-mediated.

In rats it has been shown that Type I fibres possess a higher content of GLUT-4, which is a glucose transporter protein isoform responsible for facilitated glucose uptake across the cell membrane, compared with Type II fibres (Henrikssen et al. 1990). It is not surprising, therefore, that a high positive correlation has been observed between insulin-stimulated glucose uptake and GLUT-4 content in rat muscle (Brozinick et al. 1992). In the present study, higher insulin concentration was observed at $20 \mathrm{~min}$ of exercise in the CHO trial compared with $\mathrm{W}$. It is of interest to note, however, that the glucose and insulin responses to $\mathrm{CHO}$ ingestion were moderate, yet muscle glycogen utilization was significantly reduced. This is consistent with the results of Bergstrom and Hultman (1967) who reported a decreased glycogen utilization as a result of glucose infusion-induced hyperglycaemia; but in contrast to the findings by Coyle et al. (1991) where hyperglycaemia failed to induce a glycogen sparing effect.

In conclusion, the results of this study show that drinking a $5.5 \% \mathrm{CHO}-$ electrolyte solution before and during a $60-\mathrm{min}$ treadmill run at $70 \% \mathrm{VO}_{2}$ 
max decreases glycogen utilization compared with water ingestion. Moreover, this sparing of muscle glycogen is restricted to the Type I muscle fibres. 


\section{CARBOHYDRATE INGESTION AND MUSCLE GLYCOGEN DEPLETION PATTERN IN DIFFERENT FIBRE TYPES DURING EXHAUSTIVE RUNNING}

\section{Z.1 INTRODUCTION}

In previous studies, using running as the mode of exercise, carbohydrate ingestion, compared with water, improved endurance performance over a $30-\mathrm{km}$ road race (Tsintzas et al. 1993), a $42.2-\mathrm{km}$ treadmill time trial (Chapter 4), and endurance capacity expressed as running time to exhaustion (Chapter 5).

As far as the mechanism underlying this improvement in endurance capacity and performance is concerned, it has been suggested that carbohydrate ingestion delays the onset of fatigue by either preventing the decline in blood glucose concentration and carbohydrate oxidation rate late in exercise (Coyle et al. 1986), or by decreasing the rate of muscle glycogen utilization (Bjorkman et al. 1984; Erickson et al. 1987). In our previous study, sparing of muscle glycogen was demonstrated as a result of ingesting a $5.5 \% \mathrm{CHO}$ solution during $60 \mathrm{~min}$ of constant pace treadmill running (Chapter 6). This $\mathrm{CHO}$ solution was the same as the one used in Studies 1 and 2 (Chapters 4 and 5). Furthermore, it was shown that this decrease in glycogen utilization was confined mainly to Type I fibres. Since Type I fibres are the main fibres recruited during prolonged exercise of a submaximal nature (Vollestad et al. 1984), it was hypothesized that a reduced glycogen utilization in Type I fibres is associated with the delay of the onset of fatigue when carbohydrate is ingested during exercise.

Sparing of muscle glycogen would only explain an improvement in endurance capacity if glycogen depletion was the main cause of fatigue during prolonged submaximal exercise. Indeed, glycogen depletion has been shown to coincide with fatigue during prolonged cycling exercise (Hermansen et al. 1967). However, during running exercise, mixed muscle glycogen does not appear to deplete at the point of exhaustion (Costill et al. 1971; Costill et al. 1973a; Karlsson and Saltin, 1971; Madsen et al. 1990). These results suggest that muscle glycogen depletion may not be a major 
contributor to fatigue (Madsen et al. 1990). On the other hand, studies using histochemical methods to differentiate between the glycogen content in Type I and Type II fibres have shown a selective glycogen depletion in Type I fibres to occur during prolonged running exercise (

Costill et al. 1973a). Thus, the main aim of this study was to determine the pattern of glycogen depletion in different types of fibres at the point of exhaustion during prolonged running exercise with and without carbohydrate ingestion. 


\subsection{METHODS}

Subjects: Eight male, recreational runners volunteered to participate in this study. The mean age, height, weight, $\mathrm{VO}_{2} \max$ and $\mathrm{HR} \max$ of the subjects were $29.7( \pm 1.7)$ years ( \pm SEM), $178.0( \pm 1.0) \mathrm{cm}, 74.1( \pm 3.1) \mathrm{kg}, 61.8$ $( \pm 2.3) \mathrm{ml} . \mathrm{kg}^{-1} \cdot \mathrm{min}^{-1}$ and $192.6( \pm 1.9) \mathrm{bt.min}-1$, respectively.

Preliminary measurements: Following familiarization with treadmill running and experimental procedures, the subjects undertook two preliminary tests in order to determine: (i) the relationship between running speed and oxygen uptake, and (ii) the maximum oxygen uptake ( $\mathrm{VO}_{2}$ max). The protocols of these tests have been described previously (see Chapter 3). Prior to the first experimental trial, the subjects undertook a $60-90$ min treadmill run at $70 \% \mathrm{VO}_{2}$ max in order to fully familiarized themselves with the drinking pattern and the measurements used during the experimental trials.

Protocol of the study: The subjects were required to run to exhaustion at $70-75 \% \mathrm{VO}_{2}$ max on a motorized treadmill on two different occasions separated by one week (Fig. 7.1). On the first occasion placebo (PL) was ingested throughout the run. On the second occasion a $5.5 \%$ carbohydrateelectrolyte solution ( $\mathrm{CHO}$ ) was ingested throughout the run. A random order of trials was not employed because the main aim of the study was to investigate the metabolic environment within the muscle not only at the point of exhaustion when subjects ingested placebo or carbohydrate, but also at that point during the $\mathrm{CHO}$ trial that coincided with placebo exhaustion. Thus, the experiment was conducted in a single-blind design. The 5.5\% CHO solution was chosen because in the previous studies the ingestion of the same solution, unlike water, enhanced both endurance performance over a $42.2 \mathrm{~km}$ treadmill time trial (Chapter 4) and endurance capacity (Chapter 5 ). The same solution was also shown to spare muscle glycogen over a 60 -min run at $70 \% \mathrm{VO}_{2} \max$ (Chapter 6).

Both experimental trials were performed after 3 days on a normal standardised diet and a 12-16 hour overnight fast (see Chapter 3). A warmup which consisted of a 5 -min run at $60 \% \mathrm{VO}_{2}$ max preceded each trial. Following the warm-up, the treadmill was stopped for $5 \mathrm{~min}$ and the subject consumed $8 \mathrm{ml} \cdot \mathrm{kg}^{-1}$ body weight (bw) of the assigned fluid while 
standing on the treadmill. A further $2 \mathrm{ml} . \mathrm{kg}^{-1}$ was ingested by the subjects every $20 \mathrm{~min}$ of the run. Immediately after this $5 \mathrm{~min}$ period, the treadmill running speed was increased to speed equivalent to $70 \% \cdots \mathrm{VO}_{2}$ max. In the first run, which was always a placebo trial, muscle samples were obtained at rest and immediately after the completion of the run. Venous blood samples were obtained at rest, $10 \mathrm{~min}, 20 \mathrm{~min}$ and every $20 \mathrm{~min}$ of the run thereafter, and at the point of exhaustion. One minute expired air samples were taken at $10 \mathrm{~min}, 20 \mathrm{~min}$ and every $20 \mathrm{~min}$ of the run thereafter, and during the last min of the run. The Borg scale of perceived exertion (Borg, 1973) was also used at the same intervals. Following expired air and blood sampling, the runners drank the placebo solution every $20 \mathrm{~min}$ of the run. Wet sponges were available to the subjects to be used ad libitum throughout the run. Heart rate was recorded continuously during the run. The subject's pre-exercise and post-exercise body weight in nude was also obtained.

During the second trial ( $\mathrm{CHO}$ trial) exactly the same procedures were followed. An expired air sample and a venous sample were obtained at the point which coincided with the point of exhaustion in the first trial. At this point the treadmill was stopped for $2-3 \mathrm{~min}$ and the subject was quickly transferred to an adjacent couch where a muscle sample was obtained from the vastus lateralis. Following this, the subject quickly resumed his $70-75 \% \mathrm{VO}_{2}$ max speed on the treadmill until exhaustion. The last expired air sample and a $10 \mathrm{ml}$ venous blood sample were collected during the last minute of the run (exhaustion point). Immediately after the completion of the run a third muscle sample was obtained.

Dry bulb temperatures within the laboratory were $19.8 \pm 0.3{ }^{\circ} \mathrm{C}$ and $18.4 \pm$ $0.3^{\circ} \mathrm{C}$ during the $\mathrm{PL}$ and $\mathrm{CHO}$ trials respectively. Relative humidity for the two conditions were $48.9 \pm 2.4 \%$ and $53.6 \pm 2.6 \%$ respectively.

Analysis: Expired air samples were collected and analysed as previously described (see Chapter 3). Venous blood samples were analysed for blood lactate, glucose and haemoglobin concentrations, and haematocrit values; plasma FFA and glycerol concentrations; and serum insulin, sodium and potassium concentrations. Percentage changes in plasma volume were measured as previously described (see Chapter 3 ). 
Muscle biopsy samples were quickly removed from the needle and immediately immersed in liquid nitrogen until they were freeze dried and stored at $-70^{\circ} \mathrm{C}$. At a later date one part of the freeze-dried muscle was dissected free of visible blood and connective tissue, powdered and washed twice with petroleum ether to remove the fat. Muscle metabolites (Glycogen, Glucose, G-6-P, ATP, PCr and $\mathrm{Cr}$ ) were extracted and determined enzymatically as previously described (see Chapter 3 ). The second part of the freeze-dried muscle, which was also washed twice with petroleum ether in order to remove the fat, was used to determine the glycogen content of single muscle fibres (see Chapter 3). Single fibre analysis was performed on the biopsy samples of six subjects.

Statistical analysis: A two-way analysis of variance (ANOVA) for repeated measures on two factors (experimental treatment and samplimg time) was used to compare differences between cardiovascular changes and metabolic responses to both trials. Times to exhaustion, body weight and plasma volume changes, and volumes of fluid ingested during the two trials were analysed by students $t$-test for correlated data. When using ANOVA any significant differences shown were assessed by applying the Tukey post-hoc test. Statistical significance was accepted at a 5\% level. Results are presented as mean \pm SEM. 
Fluid ingestions

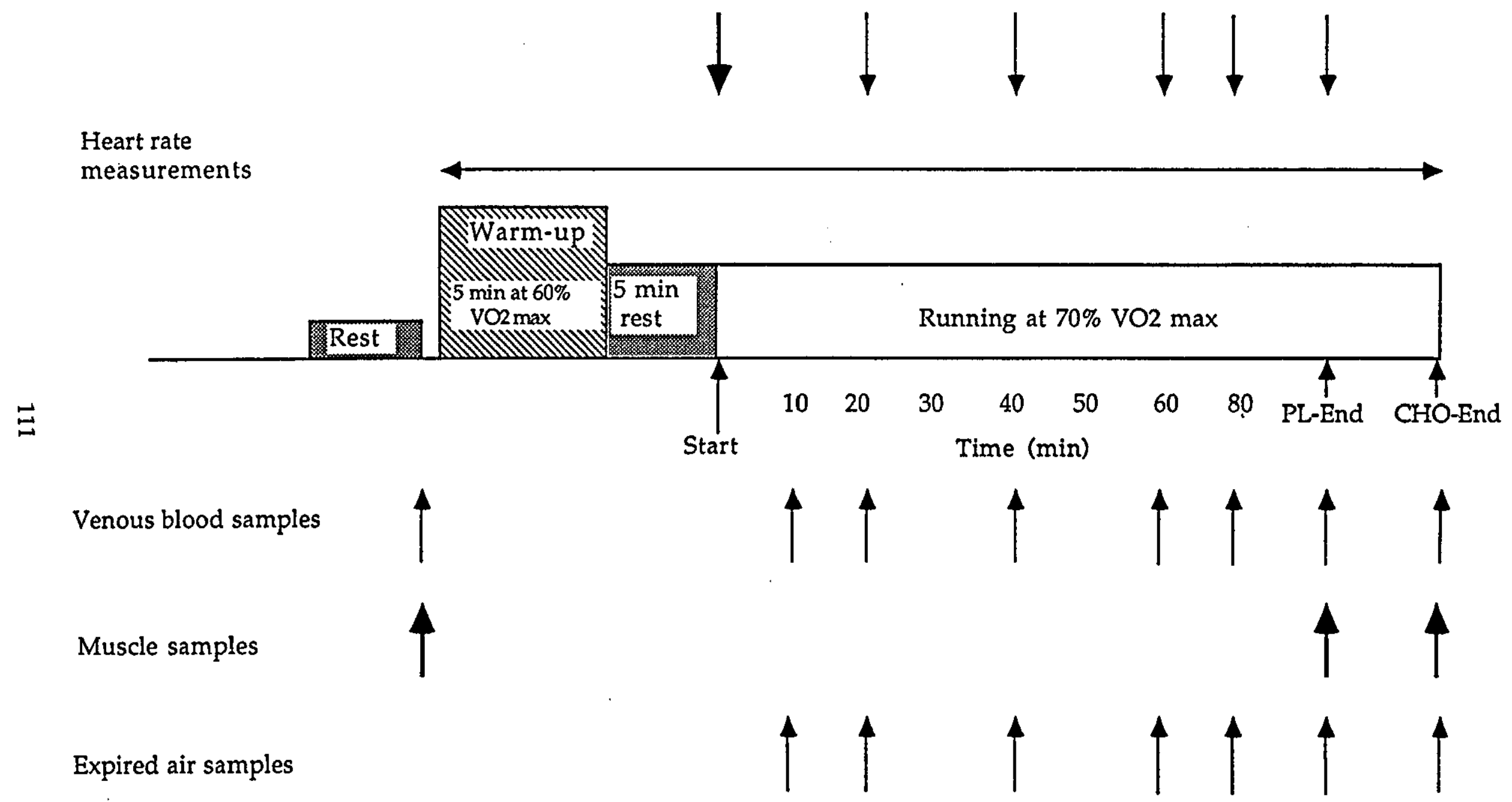




\subsection{RESULTS}

Running times to exhaustion were $104.3 \pm 8.6 \mathrm{~min}$ and $132.4 \pm 12.3 \mathrm{~min}$ in the PL and CHO trials respectively. Running time to exhaustion in the CHO trial was significantly longer $(p<0.01)$ compared with the PL trial.

Due to the longer time to exhaustion in the $\mathrm{CHO}$ trial, the total volume of fluid ingested during this trial was significantly larger $(p<0.01)$ compared with placebo ingestion ( $1328 \pm 55 \mathrm{ml}$ vs $1202 \pm 65 \mathrm{ml}$, respectively). In the CHO trial, $887 \pm 37 \mathrm{ml}$ were ingested during the first hour of the run resulting in an intake of $48.8 \pm 2.0 \mathrm{~g}$ of carbohydrate. During the last $72 \mathrm{~min}$ of the CHO trial a further $441 \pm 63 \mathrm{ml}$ of fluid were ingested, resulting in the consumption of $24.2 \pm 3.4 \mathrm{~g}$ of carbohydrate. In total, $73.0 \pm 3.0 \mathrm{~g}$ of carbohydrate were ingested throughout the $\mathrm{CHO}$ trial.

The average $\% \mathrm{VO}_{2}$ max values sustained during the $\mathrm{PL}$ and $\mathrm{CHO}$ trials were not different between trials $(76.1 \pm 0.4 \%$ vs $76.7 \pm 0.5 \%$, respectively). Neither was there any difference between trials in oxygen uptake $\left(\mathrm{VO}_{2}\right)$ and, hence, \% $\mathrm{VO}_{2} \max$ at the various sampling points (Table 7.1). Respiratory Exchange Ratio (RER) was also not different between trials (Table 7.1). Total carbohydrate and fat oxidation rates, as calculated from $\mathrm{VO}_{2}$ and RER values, were similar at all sampling points, including exhaustion, in the two trials (Table 7.1). The average carbohydrate oxidation rate was $3.18 \pm 0.10$ g.min ${ }^{-1}$ in the PL trial and $3.02 \pm 0.06$ g.min $\mathrm{min}^{-1}$ in the $\mathrm{CHO}$ trial. No difference was observed in the amount of $\mathrm{CHO}$ oxidised between the PL trial and the first $104 \mathrm{~min}$ of the CHO trial (331.7 \pm $10.4 \mathrm{~g}$ vs $314.1 \pm 6.2 \mathrm{~g}$ respectively). The total amount of $\mathrm{CHO}$ oxidised during the entire $\mathrm{CHO}$ trial was $399.8 \pm 7.9 \mathrm{~g}$.

Heart rate was not different at any sampling point between the two trials (Table 7.1). The average heart rate during the PL and $\mathrm{CHO}$ trials was $171 \pm 2$ beats. $\min ^{-1}$ and $170 \pm 2$ beats. $\mathrm{min}^{-1}$ respectively.

Body weight loss, corrected for fluid ingested, was greater in the $\mathrm{CHO}$ trial compared with PL $(3.0 \pm 0.3 \mathrm{~kg}$ vs $2.3 \pm 0.2 \mathrm{~kg}, \mathrm{p}<0.05)$. The percentage body weight loss was $4.0 \pm 0.4 \%$ and $3.2 \pm 0.3 \%$ in the CHO and PL trials respectively $(p<0.05)$. On the other hand, the percentage change in plasma volume was not different between the PL and $\mathrm{CHO}$ trials $(0.4 \pm$ 
$1.5 \%$ vs $1.8 \pm 1.3 \%$, respectively). Perceived rate of exertion was also not different between trials. In the PL trial six cases of gastrointestinal discomfort were recorded during the run to exhaustion. In contrast, four cases were observed during the CHO trial. It seems, however, that in both trials the symptoms of gastrointestinal discomfort were not severe enough to force the subjects to stop exercising.

Higher blood glucose concentrations ( $p<0.01$ ) were observed at 10 and 20 min in the CHO trial compared with the PL trial $\left(5.2 \pm 0.2 \mathrm{mmol}^{-1}\right.$ vs 3.9 $\pm 0.1 \mathrm{mmol.1}-1$ at $10 \mathrm{~min}$ and $5.2 \pm 0.3 \mathrm{mmol.} \mathrm{l}^{-1}$ vs $4.0 \pm 0.2 \mathrm{mmol} . \mathrm{l}^{-1}$ at 20 min, respectively). Serum insulin concentration (Fig. 7.3) followed a similar pattern to the blood glucose concentration being higher $(p<0.01)$ at 10 and $20 \mathrm{~min}$ in the CHO trial compared with PL (10.6 $\pm 2.8 \mathrm{mIU}^{-1}$ vs $4.5 \pm 0.4 \mathrm{mIU}^{-\mathrm{l}^{-1}}$ at $10 \mathrm{~min}$ and $10.6 \pm 2.1 \mathrm{mIU}^{-1}$ vs $3.7 \pm 0.5 \mathrm{mIU} . \mathrm{l}^{-1}$ at 20 min, respectively). No significant difference was found in blood glucose concentrations (Fig. 7.2) and serum insulin concentrations (Fig. 7.3) from 40 min until exhaustion between the two trials .

Blood lactate, serum sodium and serum potassium concentrations were not significantly different between the two trials (Table 7.2). In both trials, all three metabolites were significantly elevated at the point of exhaustion compared with rest $(p<0.01)$. Interestingly, a significant rise in serum potassium concentration was also observed at $10 \mathrm{~min}$ of the run in both trials compared with rest $(p<0.01)$.

On the other hand, plasma FFA (Fig. 7.4) and plasma glycerol (Fig. 7.5) concentrations were significantly higher $(p<0.01)$ from 20 min until exhaustion in the PL trial compared with the $\mathrm{CHO}$ trial. It is of interest to note that in both trials plasma FFA concentrations at $10 \mathrm{~min}$ were lower $(p<0.01)$ compared with the resting values. In the $\mathrm{CHO}$ trial it was not until the $104 \mathrm{~min}$ point (the point coinciding with exhaustion during the placebo trial) that the FFA concentration rose above resting values, whereas in the PL trial it occurred after $80 \mathrm{~min}$ (Fig. 7.4). On the other hand, plasma glycerol concentrations rose above resting values at $20 \mathrm{~min}$ in the PL trial and at $40 \mathrm{~min}$ in the $\mathrm{CHO}$ trial (Fig. 7.5).

As figure 7.6 shows, higher glycogen concentration was observed in the CHO trial (at the point coinciding with exhaustion during the PL trial) 
when compared with the value measured at the end of the PL trial (125.6 \pm $22.3 \mathrm{mmol} . \mathrm{kg} \mathrm{dm}^{-1}$ vs $59.8 \pm 7.9 \mathrm{mmol} . \mathrm{kg} \mathrm{dm}^{-1} \mathrm{p}<0.05$, respectively). A further $76.4 \pm 14.2 \mathrm{mmol} . \mathrm{kg} \mathrm{dm}^{-1}$ of glycogen $(p<0.01)$ were utilised in the $\mathrm{CHO}$ trial from the point coinciding with placebo exhaustion (104 min) until exhaustion (132 min) (Fig. 7.6). Thus, at the point of exhaustion there was no difference in muscle glycogen concentration between the PL and CHO trials $(59.8 \pm 7.9$ mmol.kg dm-1 vs $49.2 \pm 8.1$ mmol.kg dm-1, respectively). During the first $104 \mathrm{~min}$ in the $\mathrm{PL}$ and $\mathrm{CHO}$ trials the average rates of glycogen utilization were $3.2 \pm 0.3 \mathrm{mmol} . \mathrm{kg} \mathrm{dm}^{-1} \cdot \mathrm{min}^{-1}$ and $2.5 \pm$ $0.1 \mathrm{mmol} . \mathrm{kg} \mathrm{dm}^{-1} \cdot \mathrm{min}^{-1}$ respectively $(\mathrm{p}<0.05)$. In the $\mathrm{CHO}$ trial, the average rate of glycogen utilization was maintained at $2.6 \pm 0.7 \mathrm{mmol} . \mathrm{kg}$ $\mathrm{dm}^{-1} \cdot \mathrm{min}^{-1}$ for the period between the point coinciding with placebo exhaustion (104 $\mathrm{min}$ ) and exhaustion (132 $\mathrm{min}$ ).

Resting muscle glycogen concentration in Type II fibres was higher compared with Type I fibres ( $p<0.01$ ). The sparing of mixed muscle glycogen observed in the CHO trial was almost totally restricted to Type I fibres $\left(87.1 \pm 18 \mathrm{mmol} . \mathrm{kg} \mathrm{dm}^{-1}\right.$ vs $31.6 \pm 10.3 \mathrm{mmol} . \mathrm{kg} \mathrm{dm}^{-1} \mathrm{p}<0.01$, respectively, Table 7.3). No difference in glycogen concentration of Type II fibres was observed at $104 \mathrm{~min}$ between the two trials. At the point of exhaustion in the CHO trial (132 $\mathrm{min}$ ), the glycogen concentration in Type I fibres was lower than at $104 \mathrm{~min}(p<0.05)$; but it was similar to the value observed at the end of the PL trial $\left(28.1 \pm 7.1 \mathrm{mmol} . \mathrm{kg} \mathrm{dm}^{-1}\right.$ vs $31.6 \pm 10.3$ mmol.kg dm ${ }^{-1}$, respectively). Glycogen concentration of Type II fibres was also no different at the point of exhaustion between the two trials (Table 7.3).

During the PL trial the average rates of glycogen utilization in Type I and Type II fibres were similar $\left(2.7 \pm 0.3 \mathrm{mmol} . \mathrm{kg} \mathrm{dm}^{-1} \cdot \mathrm{min}^{-1}\right.$ vs $3.3 \pm 0.5$ mmol. $\mathrm{kg} \mathrm{dm}^{-1} \cdot \mathrm{min}^{-1}$ respectively). In the $\mathrm{CHO}$ trial, however, and for the same duration of exercise $(104 \mathrm{~min})$ the average rate of glycogen utilization in Type I fibres was lower than that in Type II fibres $(2.1 \pm 0.3 \mathrm{mmol} . \mathrm{kg} \mathrm{dm}-$ $1 . \mathrm{min}^{-1}$ vs $3.1 \pm 0.2 \mathrm{mmol} . \mathrm{kg} \mathrm{dm}^{-1} \cdot \mathrm{min}^{-1}$ respectively, $\left.\mathrm{p}<0.05\right)$. For the same period of time, the rate of glycogen utilization in Type I fibres was lower in the CHO trial compared with PL $\left(2.1 \pm 0.3 \mathrm{mmol} . \mathrm{kg} \mathrm{dm}^{-1} \cdot \mathrm{min}^{-1}\right.$ vs $2.7 \pm 0.3 \mathrm{mmol} . \mathrm{kg} \mathrm{dm}-1 \cdot \mathrm{min}^{-1}$ respectively, $\mathrm{p}<0.01$ ). In the CHO trial, the rates of glycogen utilization in Type I and Type II fibres were similar for the period between the point coinciding with placebo exhaustion (104 
min) until exhaustion ( $132 \mathrm{~min})\left(2.1 \pm 0.6 \mathrm{mmol} . \mathrm{kg} \mathrm{dm}^{-1} \cdot \mathrm{min}^{-1}\right.$ vs $2.0 \pm 1.1$ mmol.kg dm${ }^{-1} \cdot \mathrm{min}^{-1}$ respectively). The latter rates of glycogen utilization were also not different from the corresponding rates during the first 104 min.

Mixed muscle ATP, PCr, creatine, free glucose and G-6-P concentrations were not different at any sampling point between trials (Table 7.4). However, in the PL trial both ATP and PCr concentrations were lower ( $p<$ 0.05) at the point of exhaustion compared with the resting values. On the other hand, no such decrease was observed in the $\mathrm{CHO}$ trial at either the point coinciding with PL exhaustion or at exhaustion (Table 7.4). 
Table 7.1: Oxygen uptake $\left(\mathrm{VO}_{2}\right)\left(1 . \mathrm{min}^{-1}\right), \% \mathrm{VO}_{2}$ max, respiratory exchange ratio (RER), carbohydrate oxidation rate (CHO-OR) (g.min ${ }^{-1}$ ), fat oxidation rate (FAT-OR) (g.min ${ }^{-1}$ ) and heart rate (bt.min ${ }^{-1}$ ) at $10 \mathrm{~min}, 20 \mathrm{~min}, 40 \mathrm{~min}, 60 \mathrm{~min}, 80 \mathrm{~min}$, placebo exhaustion (104 $\mathrm{min}$ ) and $\mathrm{CHO}$ exhaustion (132 $\mathrm{min}$ ) in the PL and CHO trials (mean $\pm S E M, n=8$ )

\begin{tabular}{|c|c|c|c|c|c|c|c|c|}
\hline & & $\begin{array}{l}10 \\
\min \end{array}$ & $\begin{array}{l}20 \\
\min \end{array}$ & $\begin{array}{l}40 \\
\min \\
\end{array}$ & $\begin{array}{l}60 \\
\mathrm{~min} \\
\end{array}$ & $\begin{array}{l}80 \\
\mathrm{~min} \\
\end{array}$ & $\begin{array}{c}104 \\
\min \\
\end{array}$ & $\begin{array}{l}132 \\
\min \end{array}$ \\
\hline & PL & 3.4 & 3.4 & 3.5 & 3.5 & 3.5 & 3.5 & \\
\hline \multirow[t]{4}{*}{$\mathrm{VO}_{2}$} & & \pm 0.1 & \pm 0.1 & \pm 0.1 & \pm 0.1 & \pm 0.1 & \pm 0.1 & \\
\hline & $\mathrm{CHO}$ & 3.4 & 3.5 & 3.5 & 3.5 & 3.5 & 3.5 & 3.7 \\
\hline & & \pm 0.1 & \pm 0.1 & \pm 0.1 & \pm 0.1 & \pm 0.1 & \pm 0.2 & \pm 0.2 \\
\hline & PL & 74.5 & 75.2 & 76.1 & 77.2 & 76.8 & 76.9 & \\
\hline \multirow[t]{4}{*}{$\% \mathrm{VO}_{2} \max$} & & \pm 1.5 & \pm 1.5 & \pm 1.7 & \pm 1.6 & \pm 1.5 & \pm 2.3 & \\
\hline & $\mathrm{CHO}$ & 74.5 & 75.8 & 76.2 & 77.1 & 77.1 & 76.6 & 79.4 \\
\hline & & \pm 1.0 & \pm 1.3 & \pm 1.3 & \pm 0.9 & \pm 0.9 & \pm 1.2 & \pm 3.6 \\
\hline & PL & 0.95 & 0.96 & 0.95 & 0.95 & 0.92 & 0.94 & \\
\hline \multirow[t]{4}{*}{ RER } & & \pm 0.01 & \pm 0.02 . & \pm 0.02 & \pm 0.03 & \pm 0.02 & \pm 0.03 & \\
\hline & $\mathrm{CHO}$ & 0.93 & 0.91 & 0.92 & 0.92 & 0.92 & 0.93 & 0.89 \\
\hline & & \pm 0.01 & \pm 0.02 & \pm 0.01 & \pm 0.02 & \pm 0.02 & \pm 0.03 & \pm 0.02 \\
\hline & PL & 3.35 & 3.45 & 3.31 & 3.24 & 3.01 & 3.10 & \\
\hline \multirow[t]{4}{*}{ CHO-OR } & & \pm 0.22 & \pm 0.23 & \pm 0.24 & \pm 0.26 & \pm 0.35 & \pm 0.30 & \\
\hline & $\mathrm{CHO}$ & 3.08 & 3.14 & 3.03 & 3.10 & 3.12 & 3.02 & 2.66 \\
\hline & & \pm 0.26 & \pm 0.23 & \pm 0.27 & \pm 0.30 & \pm 0.42 & \pm 0.38 & \pm 0.37 \\
\hline & PL & 0.32 & 0.29 & 0.37 & 0.42 & 0.55 & 0.48 & \\
\hline \multirow[t]{3}{*}{ FAT-OR } & & \pm 0.06 & \pm 0.07 & \pm 0.10 & \pm 0.11 & \pm 0.11 & \pm 0.12 & \\
\hline & $\mathrm{CHO}$ & 0.44 & 0.52 & 0.49 & 0.49 & 0.48 & 0.52 & 0.72 \\
\hline & & \pm 0.08 & \pm 0.10 & \pm 0.09 & \pm 0.11 & \pm 0.13 & \pm 0.14 & \pm 0.13 \\
\hline \multirow[t]{4}{*}{ Heart Rate } & PL & 164 & 167 & 169 & 174 & 173 & $179 a$ & \\
\hline & & \pm 3 & \pm 3 & \pm 4 & \pm 4 & \pm 4 & \pm 4 & \\
\hline & $\mathrm{CHO}$ & 162 & 164 & 170 & 172 & 171 & 173 & $175^{a}$ \\
\hline & & \pm 3 & \pm 3 & \pm 3 & \pm 4 & \pm 4 & \pm 4 & \pm 3 \\
\hline
\end{tabular}

a $\mathrm{p}<0.01$ from rest 
Table 7.2: Blood lactate (mmol. $1^{-1}$ ), serum sodium (mmol. $1^{-1}$ ) and potassium (mmol. $1^{-1}$ ) concentrations at rest, $10 \mathrm{~min}, 20 \mathrm{~min}, 40 \mathrm{~min}, 60 \mathrm{~min}, 80 \mathrm{~min}$, placebo exhaustion (104 min) and $\mathrm{CHO}$ exhaustion (132 $\mathrm{min}$ ) in the $\mathrm{PL}$ and $\mathrm{CHO}$ trials (mean $\pm \mathrm{SEM}, \mathrm{n}=8$ )

\begin{tabular}{|c|c|c|c|c|c|c|c|c|c|}
\hline Metabolites & & Rest & $\begin{array}{l}10 \\
\min \\
\end{array}$ & $\begin{array}{l}20 \\
\min \end{array}$ & $\begin{array}{l}40 \\
\min \\
\end{array}$ & $\begin{array}{l}60 \\
\min \end{array}$ & $\begin{array}{l}80 \\
\min \\
\end{array}$ & $\begin{array}{l}104 \\
\min \end{array}$ & $\begin{array}{l}132 \\
\min \end{array}$ \\
\hline \multirow[t]{2}{*}{ i. } & PL & 0.7 & $2.2^{\mathrm{a}}$ & 2.4 & 2.6 & 2.8 & 2.4 & $3.1^{\mathrm{b}}$ & \\
\hline & & \pm 0.1 & \pm 0.4 & \pm 0.4 & \pm 0.5 & \pm 0.6 & \pm 0.4 & \pm 0.5 & \\
\hline \multicolumn{10}{|l|}{ Blood } \\
\hline \multirow[t]{4}{*}{ Lactate } & $\mathrm{CHO}$ & 0.6 & $2.2^{\mathrm{a}}$ & 2.4 & 2.2 & 2.4 & 2.4 & 2.8 & $3.0^{\mathrm{b}}$ \\
\hline & & \pm 0.1 & \pm 0.3 & \pm 0.3 & \pm 0.4 & \pm 0.4 & \pm 0.4 & \pm 0.5 & \pm 0.4 \\
\hline & PL & 140 & 141 & 141 & 142 & 142 & 142 & $143 a$ & \\
\hline & & \pm 0.4 & \pm 0.4 & \pm 0.5 & \pm 0.4 & \pm 0.5 & \pm 0.5 & \pm 0.8 & \\
\hline \multicolumn{10}{|l|}{ Serum } \\
\hline \multirow[t]{4}{*}{ Sodium } & $\mathrm{CHO}$ & 140 & 141 & 141 & 142 & 142 & 142 & 142 & $144^{a}$ \\
\hline & & \pm 0.3 & \pm 0.4 & \pm 0.4 & \pm 0.4 & \pm 0.3 & \pm 0.3 & \pm 0.4 & \pm 0.5 \\
\hline & PL & 4.3 & $5.3 \mathrm{a}$ & 5.5 & 5.6 & 5.7 & 5.8 & $5.9 \mathrm{a}$ & \\
\hline & & \pm 0.1 & \pm 0.1 & \pm 0.1 & \pm 0.1 & \pm 0.2 & \pm 0.2 & 0.3 & \\
\hline \multicolumn{10}{|l|}{ Serum } \\
\hline \multirow[t]{2}{*}{ Potassium } & $\mathrm{CHO}$ & 4.3 & $5.3^{\mathrm{a}}$ & 5.3 & 5.4 & 5.5 & 5.6 & 5.7 & $5.6^{\mathrm{a}}$ \\
\hline & & \pm 0.1 & \pm 0.1 & \pm 0.1 & \pm 0.1 & \pm 0.2 & \pm 0.2 & \pm 0.2 & \pm 0.2 \\
\hline
\end{tabular}

a $\mathrm{p}<0.01$ from rest

$\mathrm{b} \mathrm{p}<0.05$ from $10 \mathrm{~min}$ 
Table 7.3: Muscle glycogen concentrations (mmol.kg dm${ }^{-1}$ ) in Type I fibres and Type II fibres at rest and exhaustion in the PL trial and at rest, the time coinciding with PL exhaustion, and exhaustion in the CHO trial (mean $\pm S E M, n=6$ ).

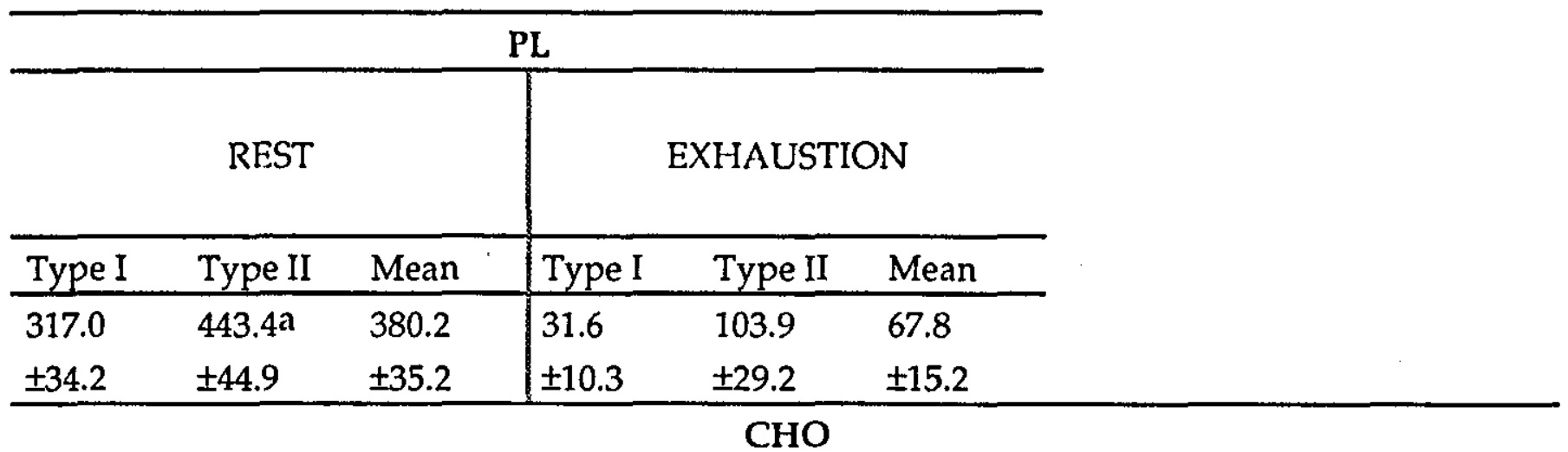

\begin{tabular}{|c|c|c|c|c|c|c|c|c|}
\hline \multicolumn{3}{|c|}{ REST } & \multicolumn{3}{|c|}{$\begin{array}{l}\text { TIME COINCIDING WITH } \\
\text { PL EXHAUSTION }\end{array}$} & \multicolumn{3}{|c|}{ EXHAUSTION } \\
\hline Type I & Type II & Mean & Type I & Type II & Mean & Type I & Type II & Mean \\
\hline 302.2 & $461.9 a$ & 382.1 & $87.1^{*}$ & 137.2 & 112.1 & $28.1^{b}$ & 79.9 & 54.0 \\
\hline \pm 34.5 & \pm 40.2 & \pm 34.5 & \pm 18.0 & \pm 41.2 & \pm 28.1 & \pm 7.1 & \pm 17.3 & \pm 10.5 \\
\hline
\end{tabular}

$*=p<0.01$ from PL $\quad a=p<0.01$ from Type I $\quad b=p<0.05$ from time coinciding with PL exhaustion 
Table 7.4 : Muscle metabolite concentrations (mmol. $\mathrm{kg} \mathrm{dm}^{-1}$ ) at rest and exhaustion in the PL trial and at rest, the time coinciding with PL exhaustion, and exhaustion in the $\mathrm{CHO}$ trial (mean $\pm S E M, n=8$ ).

\begin{tabular}{l|cc|ccc}
\hline & \multicolumn{2}{|c|}{ PL } & \multicolumn{2}{c}{ CHO } \\
\hline & & & & \multicolumn{2}{c}{$\begin{array}{c}\text { Time coinciding } \\
\text { with PL } \\
\text { Metabolites }\end{array}$} \\
& Rest & Exhaustion & Rest & Exhaustion \\
\hline ATP & & & $25.4 \pm 0.7$ & $25.0 \pm 0.8$ \\
PCr & $25.4 \pm 1.2$ & $23.8 \pm 1.2 \mathrm{~b}$ & $25.3 \pm 1.1$ & $71.5 \pm 3.9$ & $69.6 \pm 3.1$ \\
CREATINE & $81.3 \pm 2.7$ & $57.5 \pm 7.5 \mathrm{~b}$ & $79.0 \pm 3.4$ & $50.9 \pm 3.5 \mathrm{c}$ & $60.5 \pm 4.7 \mathrm{c}$ \\
GLUCOSE & $35.4 \pm 3.7$ & $63.9 \pm 5.6 \mathrm{c}$ & $31.7 \pm 3.1$ & $3.9 \pm 0.6$ & $3.3 \pm 0.5$ \\
G-6-P & $1.9 \pm 0.1$ & $4.7 \pm 1.3$ & $2.0 \pm 0.2$ & $3.1 \pm 0.3$ & $3.9 \pm 0.7$ \\
\hline
\end{tabular}

${ }^{*} \mathrm{p}<0.05$ from $\mathrm{PL}$

a $\mathrm{p}<0.01$ from time coinciding with PL exhaustion

$\mathrm{b} p<0.05$ from rest

$\mathrm{cp}<0.01$ from rest 


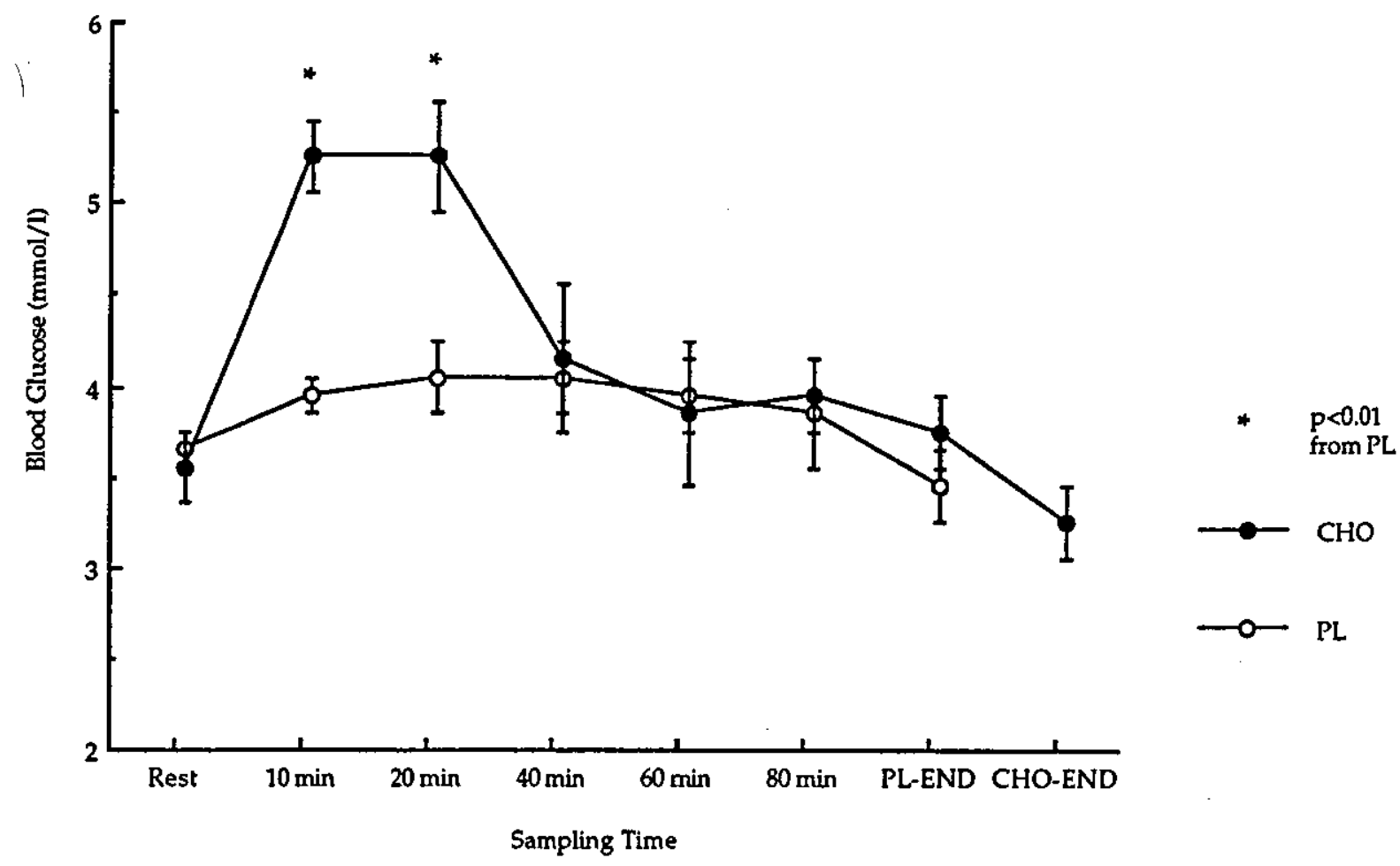

Fig. 7.2: Blood glucose concentrations (mmol/1) during the $\mathrm{PL}$ and $\mathrm{CHO}$ trials (mean \pm SEM, $n=8$ )

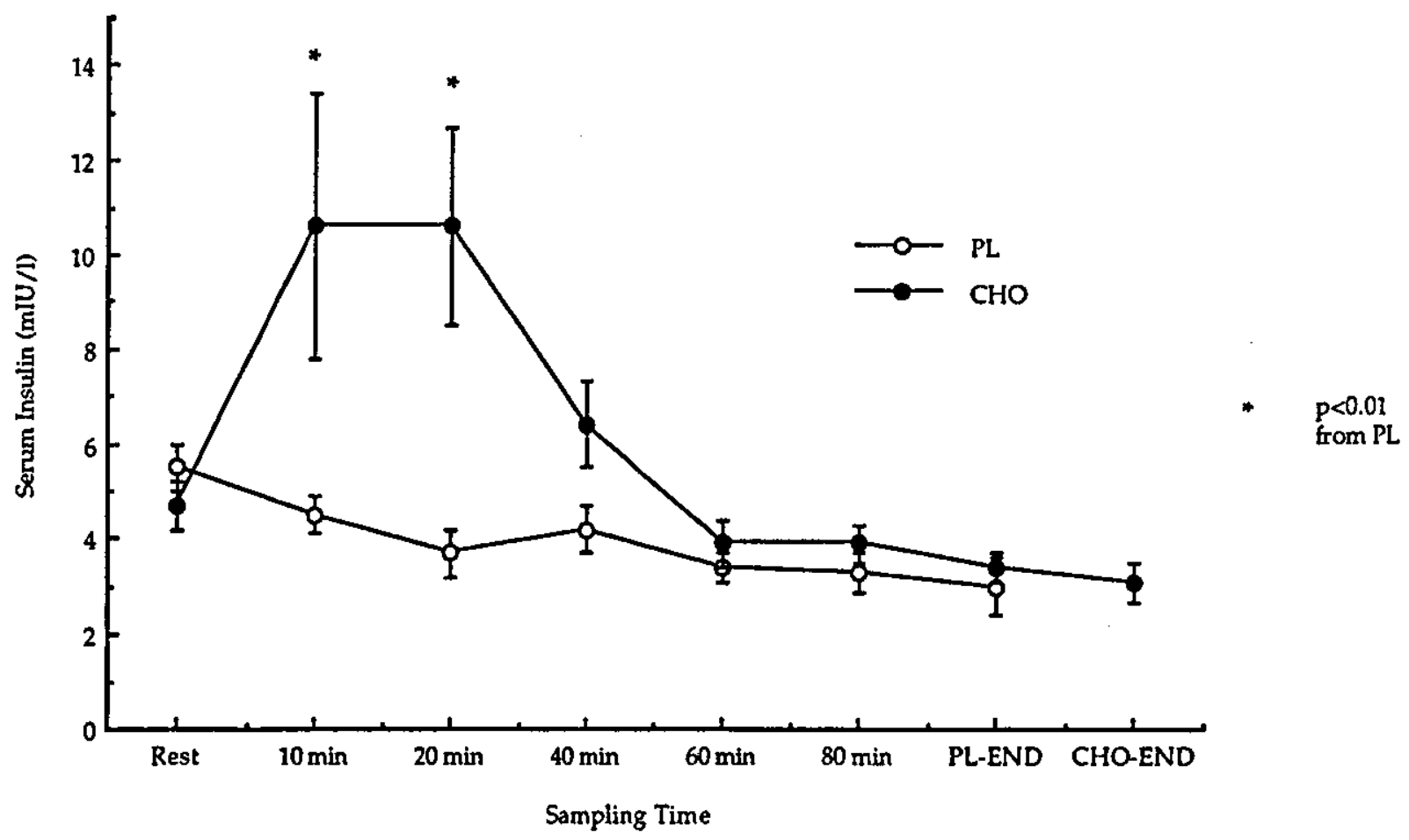

Fig. 7.3: Serum insulin concentrations (mIU/l) during the $\mathrm{PL}$ and $\mathrm{CHO}$ trials (mean \pm SEM, $n=8$ ) 


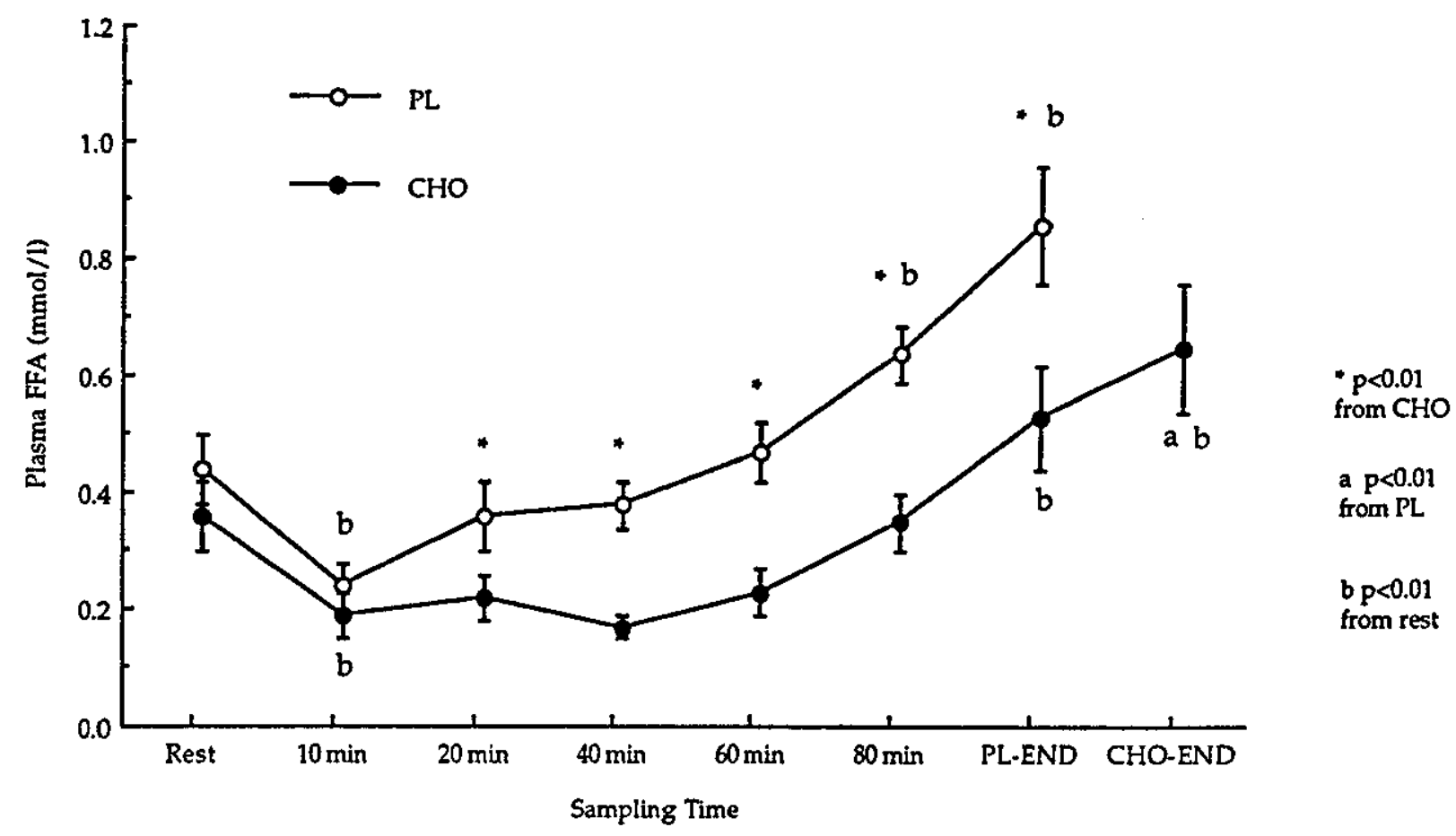

Fig. 7.4: Plasma Free Fatty Acids (FFA) concentrations (mmol/) in the PL and CHO trials (mean \pm SEM, $n=8$ )

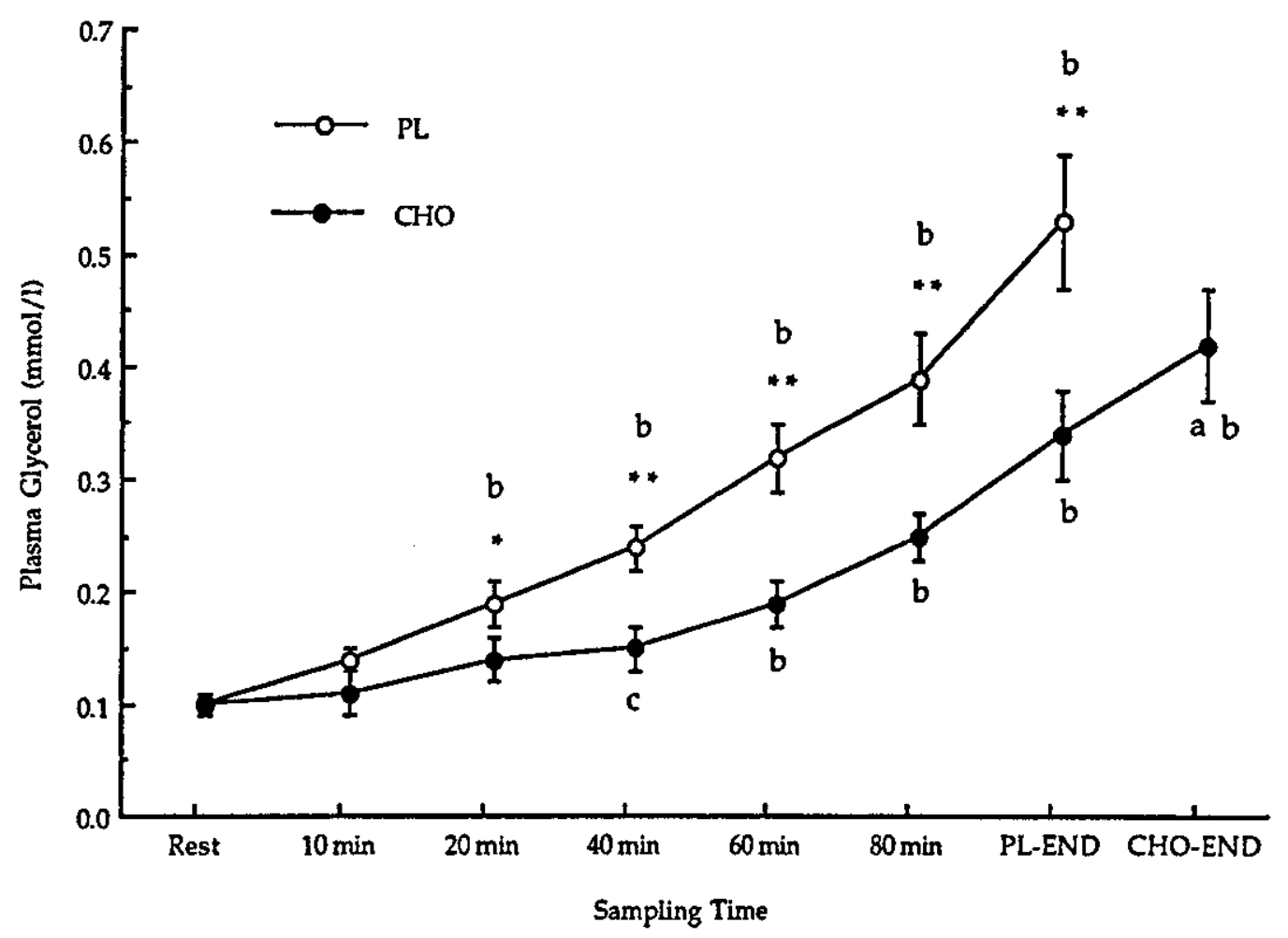

* $\mathrm{p}<0.05$ from $\mathrm{CHO}$

** $p<0.01$ from $\mathrm{CHO}$

a p $<0.05$ from PL.

b $\mathrm{p}<0.01$ from rest

Fig. 7.5: Plasma glycerol concentrations (mmol//) in the PL and CHO trials (mean $\pm S E M, n=8$ ) 


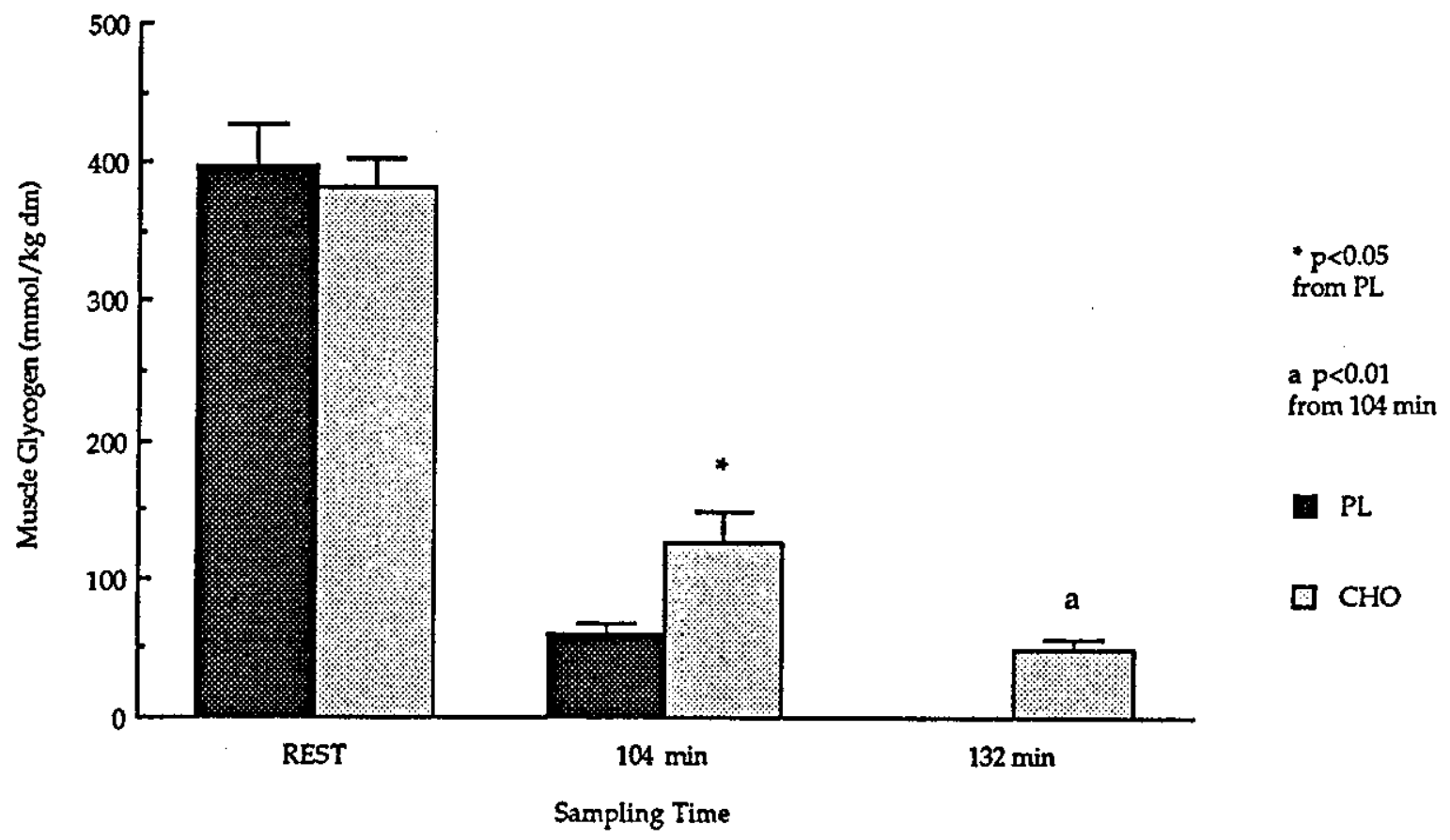

Fig. 7.6: Muscle glycogen concentrations (mmol/kg dm) at rest, PL exhaustion (104 min) and $\mathrm{CHO}$ exhaustion (132 $\mathrm{min}$ ) (mean $\pm S E M, n=8)$ 


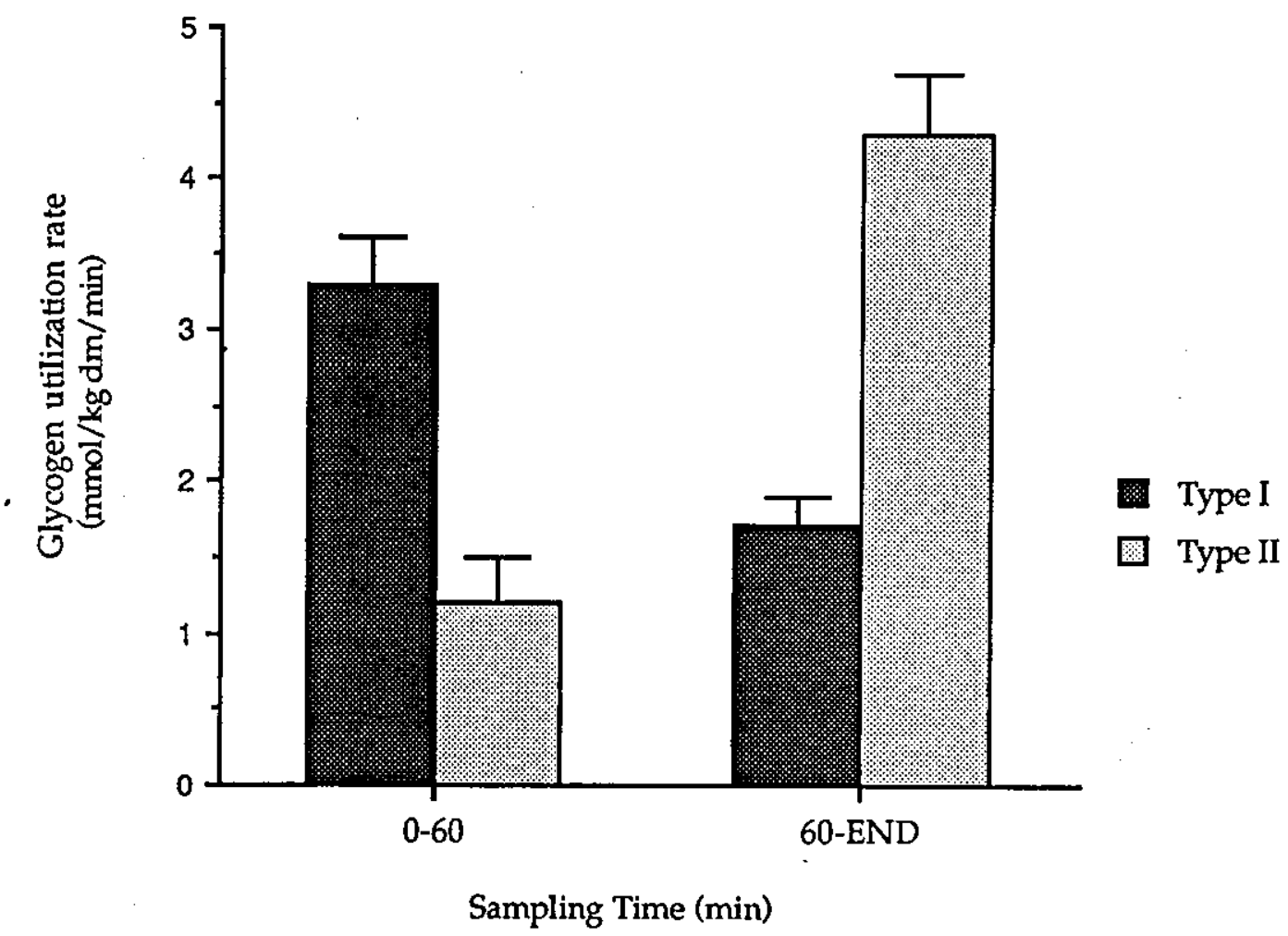

Fig. 7.7: Glycogen utilization rates in Type I and Type II fibres during prolonged running exercise without $\mathrm{CHO}$ ingestion (mean $\pm S E M, n=6$ ) (Combined data from this study and study 3 ).

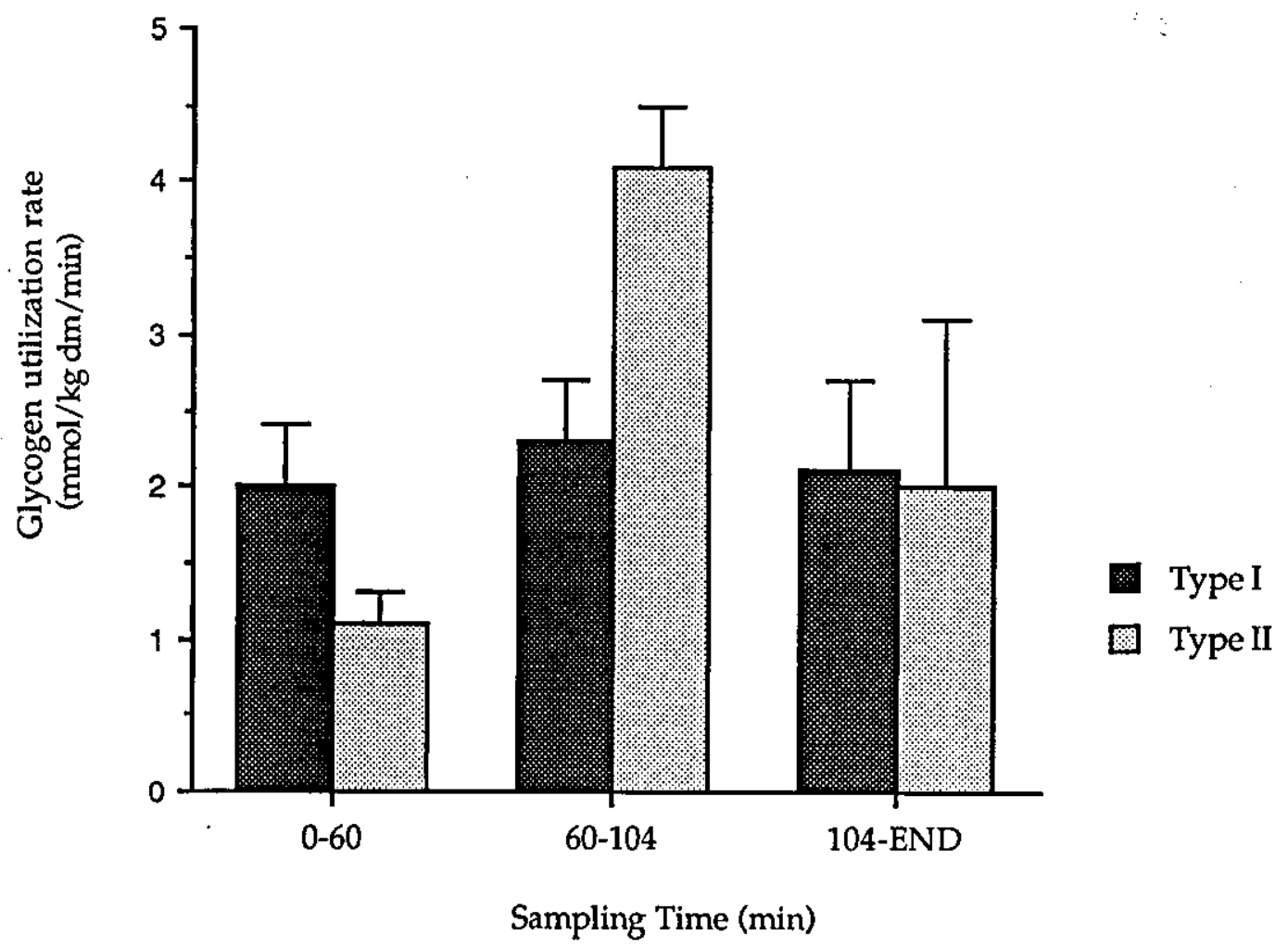

Fig. 7.8: Glycogen utilization rates in Type I and Type II fibres during prolonged running exercise with $\mathrm{CHO}$ ingestion (mean $\pm S E M, n=6$ ) (Combined data from this study and study 3 ). 


\section{Z4DISCUSSION}

There were three main findings from this study. First, a $27 \%$ improvement in endurance capacity was observed as a result of carbohydrate ingestion compared with placebo. Second, a higher muscle glycogen concentration was found at the point coinciding with placebo exhaustion in the $\mathrm{CHO}$ trial compared with the value measured at the point of exhaustion in the PL trial. This sparing of muscle glycogen was almost totally confined to Type I fibres and, thus, allowed the subjects to exercise an additional half an hour when carbohydrate was ingested. Third, a selective depletion of glycogen in Type I fibres was demonstrated following running exercise to exhaustion with or without carbohydrate ingestion.

The improvement in endurance capacity observed in the present study confirms the findings from a previous study (Chapter 5) and studies from other laboratories (Coyle et al. 1983; Coyle et al. 1986; Bjorkman et al. 1984; Maughan et al. 1989; Coggan and Coyle, 1989; Wright et al. 1991; Sasaki et al. 1987a; Macaraeg, 1983).

In a previous study (Chapter 6) a reduced glycogen breakdown in Type I fibres was observed as a result of $\mathrm{CHO}$ compared with placebo ingestion during 60 minutes of running at an intensity similar to that used in the present study, with the same CHO solution, the same drinking pattern, and subjects of similar training status. The extent of glycogen sparing after $60 \mathrm{~min}$ in the earlier study was comparable to the one observed after 104 min in the present study. It is not unreasonable, therefore, to suggest that during exercise with $\mathrm{CHO}$ ingestion almost the entire sparing of glycogen in Type I fibres occurs during the first $60 \mathrm{~min}$ of exercise and it is still present at the point coinciding with exhaustion during the placebo trial. It is suggested that the higher glycogen content of Type I fibres at that point allowed the subjects to continue the exercise for an additional half an hour. The finding that depletion of muscle glycogen in Type I fibres coincided with fatigue in both trials would further support this hypothesis.

In a previous study (Chapter 5) a $14 \%$ improvement was observed in endurance capacity as a result of ingesting the same $\mathrm{CHO}$ solution as in the present study for the first hour of exercise; water was then ingested until 
exhaustion. If glycogen sparing is indeed the single factor responsible for an improvement in endurance capacity, and it does occur during the first $60 \mathrm{~min}$ of exercise, a similar improvement would be expected in endurance capacity with either continuous $\mathrm{CHO}$ ingestion throughout exercise or $\mathrm{CHO}$ ingestion restricted to the early phase of exercise (i.e. 60 $\min )$. However, the $14 \%$ improvement in endurance capacity observed when $\mathrm{CHO}$ was fed early in exercise (Chapter 5) is almost half of the improvement found in this study when $\mathrm{CHO}$ was ingested throughout exercise (27\%). Thus, providing that the order of the trials in the present study (i.e. placebo trial always being first) did not have any positive effect on endurance capacity, it seems that sparing of muscle glycogen, although the main contributor to this improvement, is not the only one. It is possible that other factors related to $\mathrm{CHO}$ ingestion from $60 \mathrm{~min}$ until exhaustion might also play a role in the delay of the onset of fatigue.

One such factor could be sparing of liver glycogen as a result of $\mathrm{CHO}$ ingestion. It has been shown that $\mathrm{CHO}$ ingestion results in a reduced liver glucose production compared with placebo ingestion (Bosch et al. 1991). This effect could be very important in terms of maintaining blood glucose homeostasis, in a situation where liver glycogen stores are already very low and the contribution of blood glucose oxidation to energy supply is increased. It should be noted that in the present study subjects exercised following a 12-16 hour overnight fast. An overnight fast has been shown to reduce liver glycogen to a large extent (Hultman and Nilsson, 1971; Nilsson and Hultman, 1973). The fact that in the present study blood glucose homeostasis was not disturbed during running without $\mathrm{CHO}$ ingestion would not support an important role of liver glycogen sparing in the delay of the onset of fatigue when $\mathrm{CHO}$ was ingested.

Another factor relating to $\mathrm{CHO}$ ingestion and its ergogenic effect could be the suppression of plasma FFA. In this study plasma FFA concentrations were lower throughout the $\mathrm{CHO}$ trial compared with placebo trial. In our previous study, however, the suppression of FFA during the first $60 \mathrm{~min}$ of exercise, following $\mathrm{CHO}$ ingestion, was reversed when water instead of $\mathrm{CHO}$ was ingested from $60 \mathrm{~min}$ until exhaustion (Chapter 5). This reversal of the FFA response resulted in similar plasma FFA concentrations at the end of the $\mathrm{CHO}$ and control trials. An increase in plasma FFA concentration has been shown to decrease glucose uptake by the working 
muscles (Hargreaves et al. 1991). Thus, by lowering the plasma FFA concentration with $\mathrm{CHO}$ ingestion throughout exercise a higher glucose uptake may be maintained by the active muscles, at a time where the contribution of blood glucose to energy supply is very high (Coggan, 1991). On the other hand, discontinuation of $\mathrm{CHO}$ ingestion after the first hour of exercise could result in lower blood glucose uptake, and in this way it might contribute to an earlier onset of fatigue compared with continuous $\mathrm{CHO}$ ingestion. The fact, however, that in both studies $\mathrm{CHO}$ oxidation rates did not decline prior to fatigue with or without $\mathrm{CHO}$ ingestion, would not fully support such a hypothesis.

Recently, $\mathrm{CHO}$ ingestion and its effect on plasma FFA concentration has also been linked with factors relating to central causes of fatigue during prolonged exercise (Davis et al. 1992). Both plasma FFA and tryptophan compete with each other for binding sites on plasma albumin molecules. A lower plasma FFA concentration would lead to an increased concentration of tryptophan in the albumin-bound state, resulting in a decreased plasma free tryptophan concentration (Curzon et al. 1973). A decreased plasma free tryptophan concentration would lead to a decreased amount of tryptophan to cross the blood-brain barrier; as tryptophan is a precursor for serotonin (5-HT) synthesis, a decreased synthesis of 5-HT might occur (Newsholme et al. 1987). Since brain serotonin has been associated with pain and arousal, it is possible that a decrease in serotonin synthesis would attenuate the effect of central fatigue on the ability to sustain the exercise intensity (Blomstrand et al. 1991). Thus, it has been suggested that $\mathrm{CHO}$ ingestion by lowering plasma FFA concentration might have an ergogenic effect on the central origin of fatigue (Davis et al. 1992). This could also explain the difference in endurance capacity observed between the continuous $\mathrm{CHO}$ ingestion on the one hand, and the $\mathrm{CHO}$ ingestion early in exercise on the other hand. It will not explain, however, the failure of the $6.9 \% \mathrm{CHO}$ solution to improve endurance capacity and performance in Studies 1 and 2 (Chapters 4 and 5), despite the similar changes in FFA mobilization and oxidation.

Nevertheless, central to the assumption that sparing of muscle glycogen is the main factor by which $\mathrm{CHO}$ ingestion during running exercise exerts its ergogenic effect, is that glycogen depletion is the main cause of fatigue 
during prolonged submaximal exercise in thermally neutral environments.

Early studies have shown that fatigue during prolonged cycling exercise to exhaustion is associated with glycogen depletion in active skeletal muscles (Hermansen et al. 1967; Ahlborg et al. 1967; Saltin and Karlsson, 1971). Thus, depletion of glycogen in the working muscles was proposed as the limiting factor during exercise of an intensity between $65-85 \% \mathrm{VO}_{2} \max$ (Saltin and Karlsson, 1971). In some studies, however, a significant amount of whole muscle glycogen remained at the end of prolonged exercise; for example, after a 30-km outdoor race (Costill et al. 1973a), a marathon race (Sherman et al. 1983), a treadmill run to exhaustion (Madsen et al. 1990) and cycling to exhaustion (Coyle et al. 1986). However, histochemical analysis of glycogen in individual fibres revealed that Type I fibres were almost glycogen depleted in some (Costill et al. 1973a; Sherman et al. 1983; Coyle et al. 1986) but not all studies (Madsen et al. 1990). On the other hand, Type II fibres showed only a small use of glycogen. The results of the present study suggest that selective. depletion of glycogen in Type I fibres, which are heavily recruited during prolonged exercise, might explain the development of fatigue during this type of exercise. Indeed, at the end of the run to exhaustion muscle glycogen in Type I fibres was very low $\left(28.1 \pm 7.1 \mathrm{mmol} . \mathrm{kg} \mathrm{dm}^{-1}\right.$ in the CHO trial and $31.6 \pm 10.3 \mathrm{mmol} . \mathrm{kg}$ $\mathrm{dm}^{-1}$ in the PL triall. Interestingly, this value is the lowest ever reported following running exercise to exhaustion.

As both muscle ATP and PCr concentrations were lower at exhaustion compared with the resting values, it seems that the depletion of glycogen in Type I fibres and the consequent decrease in glycogenolysis in these fibres resulted in the inability of these fibres to resynthesise ATP from ADP at a rate required to sustain the exercise intensity. A mismatch between ATP resynthesis and utilization would result in increases in ADP and AMP concentrations and activation of AMP deaminase activity (Broberg and Sahlin, 1989). An increased activity of AMP deaminase would lead to an increased formation of IMP and ammonia (NH3) in the working muscles (Broberg and Sahlin, 1989; Norman et al. 1988) and a subsequent release of $\mathrm{NH}_{3}$ into the systemic circulation (Broberg and Sahlin, 1988). Although plasma $\mathrm{NH}_{3}$ concentration was not measured in the present study, in a previous study using a similar protocol, plasma $\mathrm{NH}_{3}$ 
concentration was increased by three to four times the resting value at the end of prolonged exhaustive exercise (Chapter 5). At the end of such exercise an increased IMP concentration has also been observed in glycogen depleted muscle fibres (Norman et al. 1988). Indeed, a significant inverse relationship between IMP and glycogen concentrations has been shown to exist during prolonged exercise (Spencer et al. 1991). The decreased cellular phosphate potential (ATP/[ADP*Pi]) observed under such conditions has been related to the impairment of the contractile process and the subsequent development of fatigue (Sahlin et al. 1990).

In the presence of a declining rate of glycogen utilization in Type I fibres during the latter stages of exercise without $\mathrm{CHO}$ ingestion (see fig. 7.7), a sustained total $\mathrm{CHO}$ oxidation rate throughout exercise would reflect a greater blood glucose oxidation in Type I fibres (Gollnick et al. 1981). It seems, however, that neither increased blood glucose uptake by the active muscles nor an increased rate of glycogen utilization by Type II fibres could compensate for the decreased availability of glycogen in Type I fibres and thus delay the onset of fatigue in the placebo trial.

Indeed, combining the glycogen data from this study and from a previous study where muscle samples were obtained after $60 \mathrm{~min}$ of exercise (Chapter 6), revealed an almost four times higher rate of glycogen utilization in Type II fibres during the second hour of exercise compared with the first hour. On the other hand, the corresponding rate in Type I fibres was almost three times lower during the second hour of exercise compared with the first hour (Fig. 7.7). Carbohydrate ingestion, however, decreased the rate of glycogen utilization in Type I fibers during the first hour of exercise but did not affect it from that point onwards; glycogen utilization was maintained in Type I fibres right until exhaustion (Fig. 7.8). A slight, but not significant, drop in the rate of glycogen utilization in Type II fibres was observed after $104 \mathrm{~min}$ of exercise. In the presence of ample amounts of glycogen in these fibres, the reason behind this decrease is not known. It could explain, however, the apparent inability of these fibres to fully compensate for the decreased contribution of Type I fibres to energy supply.

On the other hand, following $\mathrm{CHO}$ ingestion during exercise of similar duration to the placebo trial, glycogen concentration in Type I fibres was 
almost three times higher compared with placebo; glycogen concentration in Type II fibres was unaffected by $\mathrm{CHO}$ ingestion. As total $\mathrm{CHO}$ oxidation rates were similar during this period in both trials, it seems that blood glucose contribution to the energy metabolism was increased in the $\mathrm{CHO}$ trial compared with placebo. This increased blood glucose oxidation is probably the direct result of increased serum insulin and blood glucose concentration in the initial stages of exercise and an increased availability of exogenous glucose throughout the exercise bout due to $\mathrm{CHO}$ ingestion (Coggan, 1991). The higher glycogen concentration, coupled with maintained muscle ATP and $\mathrm{PCr}$ concentrations, at the point coinciding with placebo exhaustion would explain the ability of the subjects to exercise for an additional half an hour during the CHO trial compared with PL. At the point of exhaustion in the $\mathrm{CHO}$ trial glycogen depletion in Type I fibres was similar to that observed in the PL trial. Moreover, total $\mathrm{CHO}$ oxidation rate although slightly lower was not statistically significant from that found at placebo exhaustion. Thus, again despite the ability to oxidise carbohydrate at a high rate, fatigue coincided with depletion of glycogen in Type I fibres.

Recently, $\mathrm{CHO}$ ingestion during $135 \mathrm{~min}$ of cycling exercise has been shown to attenuate the decrease in hexo-monophosphates and tricarboxylic acid cycle intermediates, and the accumulation of IMP in the working muscles compared with placebo ingestion (Spencer et al. 1991). Interestingly, in that study the aforementioned metabolic effects of $\mathrm{CHO}$ ingestion were accompanied by a higher muscle glycogen concentration at the point of exhaustion (i.e. $135 \mathrm{~min}$ ); as a result, in a subsequent bout of exercise the subjects were able to exercise for an additional $22 \mathrm{~min}$ compared with only $0.6 \mathrm{~min}$ in the placebo trial. This would further support a link between glycogen depletion and inability to resynthesize ATP at the rate of its utilization.

However, the results of the present study clearly showed that at least during running exercise glycogen depletion occurs only in Type I fibres. Thus, a possible impairment of the contractile process during this type of exercise might be restricted to these type of fibres (i.e. slow twitch) and not to the whole muscle. Under these conditions it is not known why the fast twitch fibres, which possessed a substantial amount of glycogen at the point of exhaustion, could not compensate for the loss of the contractile 
force in Type I fibres. Therefore, further research needs to concentrate on energy metabolism of different types of fibres to investigate the exact mechanism linking glycogen depletion and fatigue.

In conclusion, the results of this study showed that the ingestion of a 5.5\% $\mathrm{CHO}$ solution during exercise increased running time to exhaustion by $27 \%$ compared with placebo ingestion. The ingestion of carbohydrate was also associated with a $28 \%$ reduction in utilization of muscle glycogen in Type I fibres. Fatigue during both trials coincided with selective glycogen depletion in Type I fibres. 


\section{CHAPTER 8}

\section{GENERAL SUMMARY}

The aim of the studies in this thesis was to investigate the performance and metabolic responses to ingestion of carbohydrate-electrolyte solutions during prolonged treadmill running.

In all studies the subjects were of comparable training status, most of the experimental procedures used were similar, and most of the changes associated with $\mathrm{CHO}$ or water ingestion were consistent. The main findings from all studies were drawn together in order to (i) try to develop an experimental model relating carbohydrate and water ingestion to the ability to perform prolonged heavy running exercise and (ii) address some questions which this model can not answer.

According to this model (Fig. 8.1), running either over the marathon distance $(42.2 \mathrm{~km})$ or to exhaustion at a constant pace without $\mathrm{CHO}$ ingestion does not result in low blood glucose concentrations or the development of hypoglycaemic symptoms at the end of the exercise; a situation frequently observed at the end of exhaustive cycling. Total $\mathrm{CHO}$ oxidation rates also did not decline prior to fatigue, suggesting that total $\mathrm{CHO}$ availability was not associated with the onset of fatigue. During the first hour of running without $\mathrm{CHO}$ ingestion, glycogen utilization in Type I fibres was almost three times higher compared with that in Type II fibres. This would imply that the former are the main fibres recruited during the early phase of prolonged running. As a result, during the latter part of exercise the glycogen utilization in Type I fibres was decreased.

On the other hand, glycogen utilization in Type II fibres increased up to four times compared with the first hour of exercise reflecting an increased recruitment of this type of fibres as Type I fibres became glycogen depleted. Fatigue was associated with glycogen depletion in Type I fibres only. However, a considerable amount of glycogen was left in Type II fibres. Despite their increased recruitment during the latter stages of the exercise, their inability to fully compensate for the reduced contribution of Type I fibres to energy supply could not be explained. 


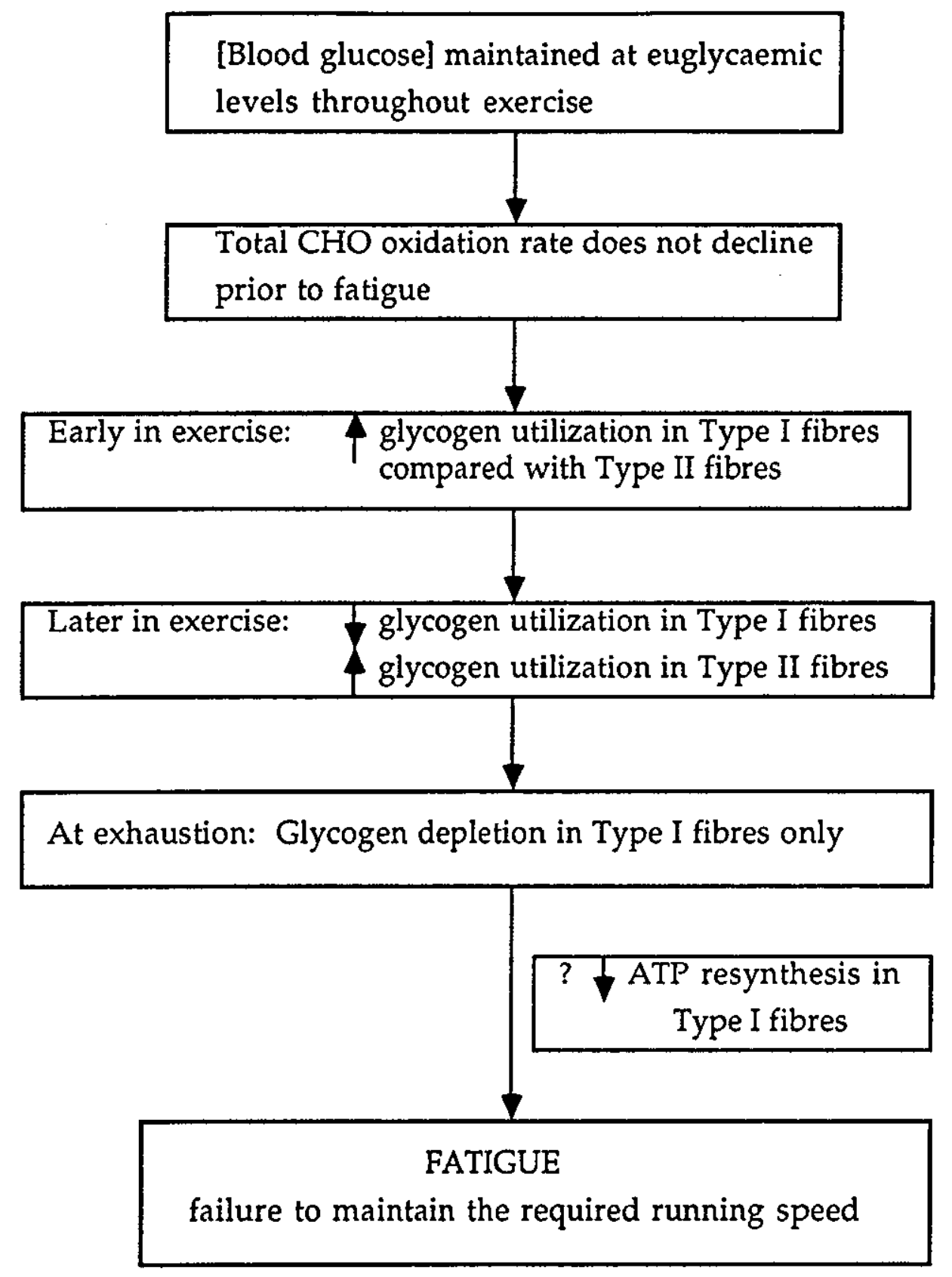

Fig. 8.1: Physiological and metabolic responses to prolonged running without $\mathrm{CHO}$ ingestion 
Compared with the control trial, the ingestion of a $5.5 \% \mathrm{CHO}$ solution resulted in a $2 \%$ faster performance time over a $42.2-\mathrm{km}$ treadmill run (Chapter 4) and a 14-27\% longer running time to exhaustion (Chapters 5 and 7). In contrast, the consumption of a $6.9 \% \mathrm{CHO}$ solution failed to induce a significant improvement in performance compared with the control (Chapters 4 and 5).

Despite an increased blood glucose and plasma insulin concentrations and a suppressed FFA and glycerol mobilization, carbohydrate ingestion during exercise did not alter total $\mathrm{CHO}$ and fat utilization rates compared with the control (Fig. 8.2). However, after $60 \mathrm{~min}$ of exercise a reduction in mixed muscle glycogen utilization was observed as a result of $\mathrm{CHO}$ ingestion when compared with the control trial. Furthermore, the ingestion of the $\mathrm{CHO}$ solution resulted in a $42 \%$ sparing of glycogen in Type I (slow twitch) fibres only. Glycogen concentration in Type II fibres was unaffected by $\mathrm{CHO}$ ingestion.

When $\mathrm{CHO}$ was ingested during exercise longer than $60 \mathrm{~min}$, this sparing of muscle glycogen in Type I fibres was still present at the point coinciding with exhaustion during the placebo trial (a mean of $104 \mathrm{~min}$ ). It is of interest to note that the amount of muscle glycogen spared in Type I fibres during the first $60 \mathrm{~min}$ of exercise was comparable to the value observed after $104 \mathrm{~min}$ of exercise $\left(84 \mathrm{mmol} . \mathrm{kg} \mathrm{dm}^{-1}\right.$ vs $70 \mathrm{mmol.kg} \mathrm{dm}-1$, respectively). These findings suggest that sparing of muscle glycogen as a result of $\mathrm{CHO}$ ingestion occurs early rather than later in exercise. This would imply that $\mathrm{CHO}$ ingestion increased blood glucose utilization right from the start of exercise (Fig. 8.2).

The spared glycogen became available during the latter stages of the $\mathrm{CHO}$ run and, therefore, could account for the $27 \%$ longer running time to exhaustion observed in Chapter 7. During this latter part of the run glycogen utilization in Type I fibres was maintained until the point of exhaustion whereas glycogen utilization in Type II fibres started to decline. Similar to the control run, the Type I fibres were glycogen depleted at the point of exhaustion whereas considerable amount of glycogen remained in Type II fibres. 


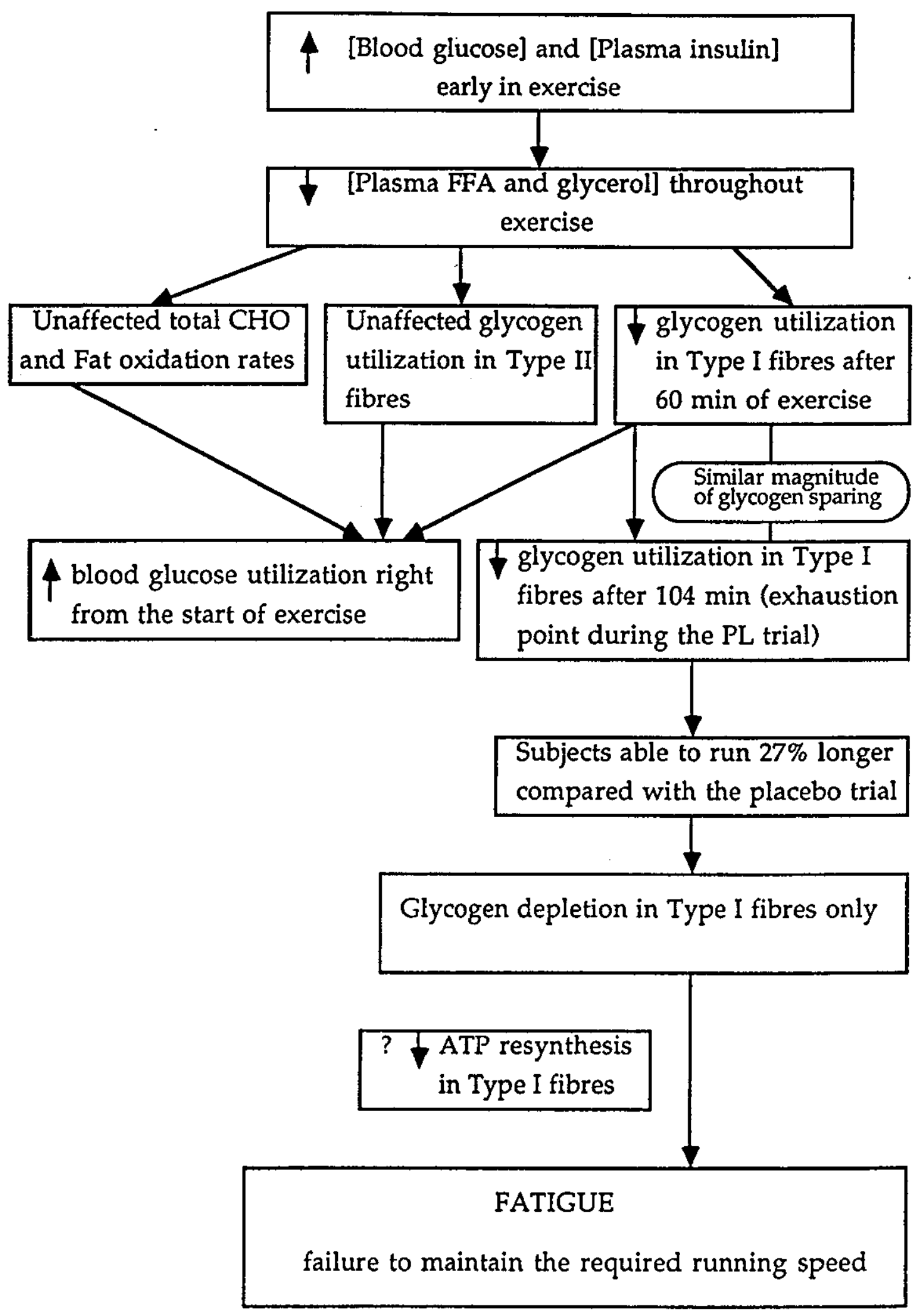

Fig. 8.2: Physiological and metabolic responses to prolonged running with $\mathrm{CHO}$ ingestion 
Irrespective of which fluid was ingested, neither blood glucose concentration nor total $\mathrm{CHO}$ oxidation rate were decreased prior to fatigue, yet the contribution of glycogen in Type I fibres to energy metabolism did decrease. These findings suggest that it was not the overall $\mathrm{CHO}$ availability which limited performance, but rather the availability of glycogen in Type I fibres. Indeed, despite the apparent ability of blood glucose and muscle glycogen in Type II fibres to supply all the $\mathrm{CHO}$ (2.5 -3.0 g. $\min ^{-1}$ ) required to sustain the exercise intensity, fatigue occurred when the glycogen was depleted in Type I fibres. This could be explained by an insufficient resynthesis of ATP in these fibres. The decreased mixed muscle ATP and PCr observed in Chapter 7 following placebo ingestion would support such a hypothesis. The absence of these changes however at the end of the $\mathrm{CHO}$ trial, yet fatigue still coincided with glycogen depletion in Type I fibres, would argue against such a hypothesis. Nevertheless, future research should concentrate on the energy metabolism of Type I fibres to understand the relationship between glycogen depletion and the onset of fatigue.

It has been suggested that blood glucose uptake during exercise is inversely related to the muscle mass involved (Richter et al. 1988). As the active muscle mass is greater during running compared with cycling, this would explain the absence of hypoglycaemia during the former compared with the latter (Coggan,1991). Thus, during running an increased provision of exogenous glucose would be aimed at preserving the limited glycogen stores rather than restoring a declining blood glucose. In cycling, however, blood glucose does not change during the first part of prolonged exercise; yet $\mathrm{CHO}$ ingestion has failed to produce a glycogen sparing in most of the cycling studies. Furthermore, the exact mechanism, by which glucose uptake by the active muscles affects glycogen utilization is at present unknown.

A wide range of $\mathrm{CHO}$ solutions of different concentration and composition have been shown to improve endurance cycling capacity. However, the few running studies conducted have produced conflicting results. In the present studies, the failure of the $6.9 \% \mathrm{CHO}$ solution to improve endurance capacity and performance might reflect the difference between running and cycling. Although this could be true, it should be reminded that the ingestion of other sports drinks of similar $\mathrm{CHO}$ concentration (7\%) 
has been shown to delay the onset of fatigue during prolonged running both in the field (Millard-Stafford et al. 1992) and in the laboratory (Wilber and Moffat, 1992). Since similar gastric emptying rates were observed between the $5.5 \%$ and $6.9 \% \mathrm{CHO}$ solutions used in the present studies, the reason behind the apparent failure of the $6.9 \%$ solution to improve endurance capacity was not clear.

It is of interest to note that a greater occurrence of gastrointestinal (GI) symptoms was associated with the ingestion of the $6.9 \%$ solution compared with the $5.5 \%$ solution. Although a direct link between fatigue and the GI symptoms was not observed, it is possible that the associated discomfort experienced by the subjects interfered with the proper function of the central nervous system resulting in a decreased central drive. The extent to which central drive for endurance running is affected by discomfort or other factors is largely unknown. It is also possible that the occurrence of GI discomfort was associated with a decreased intestinal absorption of the $6.9 \% \mathrm{CHO}$ solution. Therefore, the critical difference between the $5.5 \%$ and the $6.9 \%$ CHO-electrolyte drinks used in the present studies might not be the small difference in $\mathrm{CHO}$ concentration, but rather the effect of $\mathrm{CHO}$ composition on intestinal absorption and occurrence of GI symptoms.

It is possible that pre-exercise nutritional status could also affect the responses to $\mathrm{CHO}$ ingestion during subsequent exercise. In these studies the subjects exercised following an overnight fast preceded by a normal food intake for the days before each trial, which resulted in a normal preexercise muscle glycogen concentration. In some studies which failed to observe a glycogen sparing effect, the initial muscle glycogen concentration was almost double the values reported in the studies of this thesis; yet endurance capacity was improved. Higher initial glycogen concentration would induce a higher glycogen utilization during subsequent exercise, resulting in a high concentration of G6P. Under these conditions, glucose phosphorylation to G6P would be a limiting step for blood glucose utilization since G6P concentration is the major inhibitor of hexokinase in human skeletal muscle. This, would explain the failure of $\mathrm{CHO}$ ingestion to affect the rate of glycogen utilization in those studies with high preexercise muscle glycogen concentrations. 
Most of the studies were also performed following an overnight fast; a practice which will drastically reduce liver glycogen. In contrast, a preexercise meal would probably normalize liver glycogen content; it could also increase muscle glycogen concentration (Coyle et al. 1985). Recent studies have shown that following a CHO-rich pre-exercise meal, $\mathrm{CHO}$ ingestion during cycling (Wright et al. 1991) and running (Chryssanthopoulos et al. unpublished) does improve endurance capacity compared with placebo ingestion.

It seems that, at least during running, $\mathrm{CHO}$ ingestion impoves the capacity to perform prolonged heavy exercise by sparing the limited stores of glycogen in the working muscles. The important role of glycogen as an energy fuel during this type of exercise was highlighted by the observation that its depletion in Type I fibres coincided with the onset of fatigue. However, the possibility that some other phenomenon occuring at the same time was the true cause of fatigue under these conditions can not be excluded. 


\section{REFERENCES}

Ahlborg, J.J., Bergstrom J., Ekelund, L. G., Hultman, E. (1967): Muscle glycogen and muscle electrolytes during prolonged physical activity. Acta Physiol. Scand. 70: 129-142.

Ahlborg, G. and Felig, P. (1982): Lactate and glucose exchange across the forearm, legs, and splanchnic bed during and after prolonged leg exercise. J. Clin. Invest. 69: 45-54.

Apple, F.S., Rogers, M.A., Casal, D.C., Sherman, W.M., Ivy, J.L. (1985): Creatine kinase-MB isoenzyme adaptations in stressed human skeletal muscle of marathon runners. J. Appl. Physiol. 59: 149-153.

Aragon, J.J., Tornheim, J.K. and Lowenstein, J.M. (1980): On a possible role of IMP in the regulation of phosphorylase activity in skeletal muscle. FEBS Lett. 117, Suppl. : K56-K64.

Armstrong, L.E., Hubbard, R.W., Jones, B.H. and Daniels, J.T. (1986): Preparing Alberto Salazar for the heat of the 1984 Olympic marathon. Physician Sportsmed. 14: 73-81.

Asmussen, E. (1979): Muscle fatigue. Med. Sci. Sports Exerc. 11: 313-321.

Barr, S.I., Costill, D.L. and Fink, W.J. (1991): Fluid replacement during prolonged exercise: effects of water, saline, or no fluid. Med. Sci. Sports Exerc. 23 (7): 811-817.

Bebb, J., Brewer, J., Patton, A., Williams, C. (1984): Endurance running and the influence of diet on fluid intake. J. Sports Sci. 2: 198A.

Beckers, E.J., Rehrer, N.J., Brouns, F., Ten Hoor, F. and Saris, W.H.M. (1988): Determination of total gastric volume, gastric secretion and residual meal using the double sampling technique of George. Gut, 29: 1725-1729.

Bergstrom, J., Hermansen, L., Hultman, E., Saltin, B. (1967): Diet, muscle glycogen and physical performance. Acta Physiol. Scand. 71: 140-150. 
Bergstrom, J. (1962): Muscle electrolytes in man. Scan. J. Clin. Lab. Invest. Suppl. 68: 1-100.

Bergstrom, J. and Hultman, E. (1967): A study of glycogen metabolism during exercise in man. Scand. J. Clin. Invest. 19: 218-228.

Bergstrom, J., Hultman, E., Jorfeldt, L., Pernow, B. and Wahren, J. (1969): Effect of nicotinic acid on physical working capacity and on metabolism of muscle glycogen in man. J. Appl. Physiol. 26: 170-176.

Bjorkman, O., Sahlin, K., Hagenfeldt, L. and Wahren, J. (1984): Influence of glucose and fructose ingestion on the capacity for long-term exercise in well-trained men. Clinical Physiology, 4: 483-494.

Blomstrand, E., Hassmen, P., Ekblom, B. and Newsholme, E.A. (1991): Administration of branched-chain amino acids during sustained exerciseeffects on performance and on plasma concentration of some amino acids. Eur. J. Appl. Physiol. 63: 83-88.

Bonen, A., McDermott, J.C. and Tan, M.H. (1990) : Glucose transport in skeletal muscle. In: Taylor, A.W., Gollnick, P.D., Green, H.J., Ianuzzo, C.D., Noble, E.G., Metivier, G. and Sutton, J.R. (Eds). Biochemistry of exercise VII, Vol. 21: 295-317.

Bosch, A.N., Noakes, T.D. and Dennis, S. (1991): Carbohydrate ingestion during prolonged exercise: a liver glycogen sparing effect in glycogen loaded subjects. Med. Sci. Sports Exerc. 23 (4): S152.

Borg, G.A.V. (1973): Perceived exertion: a note on 'history' and methods. Med. Sci. Sports Exerc. 5(2): 90-93.

Broberg, S. and Sahlin, K. (1989): Adenine nucleotide degradation in human skeletal muscle during prolonged exercise. J. Appl. Physiol. 67(1): 116-122.

Broberg, S. and Sahlin, K. (1988): Hyperammonaemia during prolonged repeated submaximal exercise: an effect of glycogen depletion?. J. Appl. Physiol. 65: 2475-2477. 
Brooke, M.H. and Kaiser, K.K. (1970): Muscle fiber types: how many and what kind? Arch. Nuerol. 23: 369-379.

Brouns, F. (1991): Etiology of gastrointestinal disturbances during endurance events. Scand. J. Med. Sci. Sports, 1: 66-77.

Brozinick, J.T. Jr, Etgen, G.J. Jr, Yaspelkis, B.B. III and Ivy, J.L. (1992): Contraction-activated glucose uptake is normal in insulin-resistant muscle of the obese Zucker rat. J. Appl. Physiol. 73 (1): 382-387.

Burgess, M.L., Robertson, R.J., Davis, J.M. and Norris, J.M. (1991): PRE, blood glucose and carbohydrate oxidation during exercise: effects of glucose feedings. Med. Sci. Sports Exerc. 23 (3): 353-359.

Carruthers, A. (1990): Facilitated diffusion of glucose. Physiological Reviews, 70 (4): 1135-1176.

Chasiotis, D., Sahlin, K. and Hultman, E. (1982): Regulation of glycogenolysis in human skeletal muscle at rest and during exercise. J. Appl. Physiol. 53:708-715.

Chasiotis, D. (1985): Effect of adrenalin infusion on cAMP and glycogen phosphorylase in fast-twitch and slow-twitch rat muscle. Acta Physiol. Scand. 125: 537-540.

Christensen, E.H., Hansen, O. (1939): Arbeitsfahigkeit und Ehrnahrung. Skand. Arch. Physiol. 81: 160-175.

Chromy, V., Gergel, J., Voznicek, J., Krobholzova, L., Musil, J. (1977): Assay of serum free fatty acids by extraction, photometric procedures. Clin. Chim. Acta 80: 327-332.

Claremont, A.D., Costill, D.L., Fink, W. and Van Handel, P. (1976): Heat tolerance following diuretic induced dehydration. Med. Sci. Sports $8(4)$ : 239-243. 
Coggan, A.R. and Coyle, E.F. (1987): Reversal of fatigue during prolonged exercise by carbohydrate infusion or ingestion. J. Appl. Physiol. 63: 23882395.

Coggan, A.R. and Coyle, E.F. (1989): Metabolism and performance following carbohydrate ingestion late in exercise. Med. Sci. Sports Exerc. 21(1): 59-65.

Coggan, A.R. and Coyle, E.F. (1991): Carbohydrate ingestion during prolonged exercise: Effects on metabolism and performance. In: Holloszy, J.O. (Ed.). Exercise and sport sciences reviews. Vol. 19, 1-40.

Coggan, A.R., Spina, R.J., Kohrt, W. M., Bier, D.M. and Hollotzy, J.O. (1991): Plasma glucose kinetics in a well-trained cyclist fed glucose throughout exercise. Inter. J. Sport Nutrition, 1: 279-288.

Coggan, A.R., Kohrt, W. M., Spina, R.J., Bier, D.M. and Hollotzy, J.O. (1990): Endurance training decreases plasma glucose turnover and oxidation during moderate intensity exercise in men. J. Appl. Physiol. 68: 990-996.

Coggan, A.R. (1991): Plasma glucose metabolism during exercise in humans. Sports Med. 11 (2): 102-124.

Constantin-Teodosiu, D. (1992): Regulation of pyruvate dehydrogenase complex activity and acetyl group formation in skeletal muscle during exercise. PhD Thesis, Karolinska Institute, Sweden.

Conlee, R.K. (1987): Muscle glycogen and exercise endurance : A twentyyear perspective. Exer. Sports Sci. Revs 15 : 1-28.

Conlee, R.K., McLane, J.A., Rennie, M.J., Winder, W.W. and Holloszy, J.O. (1979): Reversal of phosphorylase activity in muscle despite continued contractile activity. Am. J. Physiol. 237 (6): R291-R296.

Cooper, H., Levitan, R., Fordtran, J.S. and Ingelfinger, F.J. (1966): A method for studying absorption of water and solute from the human small intestine. Gastroenterology 50: 1-7. 
Costill, D.L., Gollnick, P.D., Jansson, E.D., Saltin, B. and Stein, E.M. (1973a): Glycogen depletion pattern in human muscle fibres during distance running. Acta Physiol. Scand. 89: 374-383.

Costill, D.L., Bennett, A., Branam, G. and Eddy, D. (1973b): Glucose ingestion at rest and during prolonged exercise. J. Appl. Physiol. 34(6): 764769.

Costill, D.L, Sparks, K., Gregor, R. and Turner, C. (1971): Muscle glycogen utilization during exhaustive running. J. Appl. Physiol. 31: 353-356.

Costill, D.L. and Saltin, B. (1974): Factors limiting gastric emptying during rest and exercise. J. Appl. Physiol. 37(5): 679-683.

Costill, D.L., Coyle, E., Dalsky, G., Evans, W., Fink, W. and Hoopes, D. (1977): Effects of elevated plasma FFA and insulin on muscle glycogen usage during exercise. J. Appl. Physiol. 43(4): 695-699.

Costill, D.L. and Miller, J.M. (1980): Nutrition for endurance sport: carbohydrate and fluid balance. Int. J. Sports Med. 1: 2-14.

Costill, D.L., Jansson, E., Gollnick, P.D. and Saltin, B. (1974): Glycogen utilization in leg muscle of men during level and uphill running. Acta Physiol. Scand. 91: 475-481.

Coyle, E.F., Costill, D.L., Fink, W.J. and Hoopes, D.G. (1978): Gastric emptying rates for selected athletic drinks. Res. Quart. 49 (2): 119-124.

Coyle, E.F., Hagberg, J.M., Hurley, B.F., Martin, W.H., Ehsani, A.A. and Holloszy, J.O. (1983): Carbohydrate feeding during prolonged strenuous exercise can delay fatigue. J. Appl. Physiol. 55(1): 230-235.

Coyle, E.F., Coggan, A.R., Hemmert, M.K., Lowe, R.C. and Walters, T.J. (1985): Substrate usage during prolonged exercise following a preexercise meal. J. Appl. Physiol. 59: 429-433.

Coyle, E.F., Coggan, A.R., Hemmert, M.K. and Ivy, J.L. (1986): Muscle 
glycogen utilisation during prolonged strenuous exercise when fed carbohydrate. J. Appl. Physiol. 61(1): 165-172.

Coyle, E.F., Hamilton, M.T., Alonso, J.G., Montain, S.J., Ivy, J.L. (1991): Carbohydrate metabolism during intense exercise when hyperglycemic. J. Appl. Physiol. 70(2): 834-840.

Coyle, E.F., Montain, S.J. (1992a): Carbohydrate and fluid ingestion during exercise: are there trade offs? Med. Sci. Sports Exerc. 24(6): 671-678.

Coyle, E.F., Montain, S.J. (1992b): Benefits of fluid replacement with carbohydrate during exercise. Med. Sci. Sports Exerc. 24(9): S324-S330.

Curzon, G., Friedel, J. and Knott, P.J.(1973): The effect of fatty acids on the binding of tryptophan to plasma protein. Nature 242: 198-200.

Danforth, W.H. (1965): Activation of glycolytic pathway in muscle. In: Control of Energy metabolism, edited by Chance, B. and Estabrook, R.W. New York : Academic.

Davis, J.M., Lamb, D.R., Burgess, W.A. and Bartoli, W.P. (1987): Accumulation of deuterium oxide in body fluids after ingestion of $\mathrm{D}_{2} \mathrm{O}$ labeled beverages. J. Appl. Physiol. 63 (5): 2060-2066.

Davis, J.M., Lamb, D.R., Pate, R.R., Slentz, C.A., Burgess, W.A. and Bartoli, W.P. (1988a): Carbohydrate-electrolyte drinks: effects on endurance cycling in the heat. Am. J. Clin. Nutr. 48: 1023-1030.

Davis, J.M., Burgess, W.A., Slentz, C.A., Bartoli, W.P. and Pate, R.R. (1988b): Effects of ingesting $6 \%$ and $12 \%$ glucose/electrolyte beverages during prolonged intermittent cycling in the heat. Eur. J. Appl. Physiol. 57: 563-569.

Davis, J.M., Burgess, W.A., Slentz, C.A. and Bartoli, W.P. (1990): Fluid availability of sports drinks differing in carbohydrate type and concentration. Am. J. Clin. Nutr. 51: 1054-1057. 
Davis, J.M., Bailey, S.P., Woods, J.A., Galiano, F.J., Hamilton, M.T. and Bartoli, W.P. (1992): Effects of carbohydrate feedings on plasma free tryptophan and branched-chain amino acids during prolonged cycling. Eur. J. Appl. Physiol. 65: 513-519.

Deuster, P.A., Singh, A., Hofmann, A., Moses, F.M. and Chrousos, G.C. (1992): Hormonal responses to ingesting water or a carbohydrate beverage during a 2 h run. Med. Sci. Sports Exerc. 24 (1): 72-79.

Dietz, M.R., Chiasson, J.L., Soderling, T.R. and Exton, J.H. (1980): Epinephrine regulation of skeletal muscle glycogen metabolism. J. Biol. Chem., 255: 2301-2307.

Dill, D.B. and Costill, D.L. (1974): Calculation of percentage changes in volumes of blood plasma, and red cells in dehydration. J. Appl. Physiol., $37,247-248$.

Donaldson, S.K. (1990): Fatigue of sarcoplasmic reticulum: failure of excitation-contraction coupling in skeletal muscle. In: Taylor, A.W., Gollnick, P.D., Green, H.J., Ianuzzo, C.D., Noble, E.G., Metivier, G. and Sutton, J.R. (Eds). Biochemistry of exercise VII, Vol. 21: 49-57.

Douen, A.G., Ramlal, T., Klip, A., Young, D.A., Cartee, G.D. and Holloszy, J.O. (1989): Exercise-induced increase in glucose transporters in plasma membranes of rat skeletal muscle. Endocrinology 124: 304-309.

Douen, A.G., Ramlal, Rastogi, S., Bilan, P.J., Cartee, G.D., Vranic, M., Holloszy, J.O. and Klip, A. (1990): Exercise induces recruitment of the insulin responsive glucose transporter. Evidence for distinct intracellular insulin and exercise-recruitable transporter pools in skeletal muscle. J. Biol. Chem. 265: 134327-13430.

Douglas, P.S., O'Toole, M.L., Douglas, W., Hiller, B., Hackney, K. and Reichek, N. (1987): Cardiac fatigue after prolonged exercise. Circulation 76, 6: 1206-1213.

Duchman, S.M., Bleiler, T.L., Schedl, H.P., Summers, R.W. and Gisolfi, 
C.V. (1990): Effects of gastric function on intestinal composition of oral rehydration solutions. Med. Sci. Sports Exerc. 22 (Suppl. 2): S89.

Edwards, R.H.T., Harris, R.C., Hultman, E., Kaijser, L., Koh, D. and Nordesjo, L.O. (1972): Effect of temperature on muscle energy metabolism and endurance during successive isometric contractions, sustained to fatigue, of the quadriceps muscle in man. J. Physiol. Lond. 220: 335-352.

Edwards, T.L., Santeusanio, D.M. and Wheeler, K.B. (1984): Field test of the effects of carbohydrate solutions on endurance performance, selected blood serum chemistries, perceived exertion, and fatigue in world class cyclists. Med. Sci. Sports Exerc. 16:190.

Erickson, M.A., Schwartzkopf and McKenzie, R.D. (1987): Effects of caffeine, fructose and glucose ingestion on muscle glycogen utilization during exercise. Med. Sci. Sports Exerc. 19, 579-583.

Essen, B. (1978): Glycogen depletion of different fibre types in human skeletal muscle during intermittent and continuous exercise. Acta Physiol. Scand. 103: 446-455.

Felber, J.P., Acheson, K.J. and Tappy, L. (1993): From obesity to diabetes. J. Wiley and sons. Chichester.

Felig, P.A., Cherif, A., Minagawa, A. and Wahren, J. (1982): Hypoglycemia during prolonged exercise in normal men. N. Engl. J. Med., 306: 895-900.

Fenn, C.E., Leiper, J.B., Light, I.M. and Maughan, R.J. (1983): Effects of oral administration of fluid, electrolytes and substrate on endurance capacity in man. J. Physiol. 341: 66P.

Fielding, R.A., Costill, D.L., Fink, W.J., King, D.S., Hargreaves, M., Kovaleski, J.E. (1985): Effect of carbohydrate feeding frequencies and dosage on muscle glycogen use during exercise. Med. Sci. Sports Exerc. 17(4): 472476.

Fink, W.J., Costill, D.L. and van Handel, P.J. (1975): Leg muscle metabolism during exercise in the heat and cold. Eur. J. Appl. Physiol., 34: 183-190. 
Flynn, M.G., Costill, D.L., Hawley, J.A., Fink W.J., Neufer, P.D., Fielding, R.A. and Sleeper, M.D. (1987): Influence of selected carbohydrate drinks on cycling performance and glycogen use. Med. Sci. Sports Exerc. 19(1): 37-40.

Fordtran, J.S. and Saltin, B. (1967): Gastric emptying and intestinal absorption during prolonged severe exercise. J. Appl. Physiol., 23(3): 331335.

Fordtran, J.S. (1975): Stimulation of active and passive sodium absorption by sugars in the human jejunum. The Journal of Clinical Investigation, Vol. 55: 728-737.

Forster, C.D., Taylor, J. Y. and Macdonald, I.A. (1991): The assay of the catecholamine content of small volumes of human plasma. In: Rollema, H., Westerink, B., Drifjhout, W.J. (Ed.). Monitoring molecules in neuroscience. Conference on in vivo methods, 173-175.

Fortney, S.M., Wenger, C.B., Bore, J.R. and Nadel, E.R. (1984): Effects of hyperosmolality on control of blood flow and sweating. J. Appl. Physiol., 57: 1688-1695.

Fortney, S.M., Nadel, E.R., Wenger, C.B. and Bove, J.R. (1981): Effect of blood volume on sweating rate and body fluids in exercising humans. J. Appl. Physiol. 51: 1594-1600.

Foster, C., Costill, D.L. and Fink, W.J. (1980): Gastric emptying characteristics of glucose and glucose polymer solutions. Res. Quart. 51(2): 299-305.

Fruth, J.M. and Gisolfi, C.V. (1983): Effects of carbohydrate consumption on endurance performance: Fructose versus Glucose. In: Fox, E.L. (Ed). Nutrient utilization during exercise. Ross Laboratories, Columbus, 68-75.

Galbo, H., Holst, J.J., Christensen, N.J. (1975): Glucagon and plasma catecholamine responses to graded and prolonged exercise in man. J. Appl. Physiol., 38: 70-76. 
Galbo, H., Christensen, N.J., Holst, J.J. (1977): Glucose-induced decrease in glycagon and epinephrine responses to exercise in man. J. Appl. Physiol. 42: 525-530.

Galbo, H., Holst, J.J. and Christensen, N.J. (1979): The effect of different diets and of insulin on the hormonal responses to prolonged exercise. Acta Physiol. Scand., 107: 19-32.

Giles, D. and Maclaren, D. (1984): Effects of caffeine and glucose ingestion on metabolic and respiratory functions during prolonged exercise. J. Sports Sciences, 2: 35-46.

Gisolfi, C.V., Summers, R.W., Schedl, H.P., Bleiler, T.L. and Oppliger, R.A. (1990): Human intestinal water absorption: direct vs. indirect measurements. Am. J. Physiol. 258 (21): G216-G222.

Gollnick, P.D., Piehl, K., Sanbert, C.W., Armstrong, R.B. and Saltin, B. (1972): Diet, exercise, and glycogen in human muscle fibres. J. Appl. Physiol., 33: 421-425.

Gollnick, P.D., Armstrong, R.B., Saubert, C.W., Sembrowich, W.L., Shepherd, R.E. and Saltin, B. (1973): Glycogen depletion patterns in human skeletal muscle fibres during prolonged work. Pflugers Arch. 344: $1-12$.

Gollnick, P.D., Pernow, B., Essen, B. Jansson, E. and Saltin, B. (1981): Availability of glycogen and plasma FFA for substrate utilization in leg muscle of man during exercise. clin. Physiol. Oxd.1: 27-42.

Greenhaff, P.L., Ren, J.M., Soderlund, K. and Hultman, E. (1991): Energy metabolism in single human muscle fibres during contraction without and with epinephrine infusion. Am. J. Physiol. 260 (Endocrinol. Metab. 23): E713-E718.

Greenleaf, J.E. (1992): Problem: thirst, drinking behavior, and involuntary dehydration. Med. Sci. Sports Exerc. 24 (6): 645-656. 
Hamilton, M.T., Gonzalez-Alonso, J., Montain, J., Coyle, E.F. (1991): Fluid replacement and glucose infusion during exercise prevent cardiovascular drift. J. Appl. Physiol., 71(3), 871-877.

Hargreaves, M., Costill, D.L., Coggan, A., Fink, W.J., Nishibata, I. (1984): Effect of carbohydrate feedings on muscle glycogen utilization and exercise performance. Med. Sci. Sports Exerc. 16(3): 219-222.

Hargreaves, M., and Briggs, C.A. (1988): Effect of carbohydrate ingestion on exercise metabolism. J. Appl. Physiol. 65: 1533-1555.

Hargreaves, M. and Richter, E.A. (1988): Regulation of skeletal muscle glycogenolysis during exercise. Can. J. Spt. Sci., 13(4): 197-203.

Hargreaves, M., Kiens, B. and Richter, E.A. (1991): Effect of increased plasma free fatty acid concentrations on muscle metabolism in exercising men. J. Appl. Physiol. 70 (1): 194-201.

Harris, R.C., Hultman; E. and Nordesjo, L.O. (1974): Glycogen, glycogenolytic intermediates and high energy phosphates determined in biopsy samples of musculus quadriceps femoris of man at rest. Methods and variance of values. Scand. J. Clin. Lab. Invest. 33: 109-120.

Hawley, J.A., Dennis, S.C., Laidler, B.J., Bosch, A.N., Noakes, T.D. and Brouns F. (1991): High rates of exogenous carbohydrate oxidation from starch ingested during prolonged exercise. J. Appl. Physiol. 71 (5): 18011805.

Hawley, J.A., Dennis, S.C. and Noakes, T.D. (1992): Oxidation of carbohydrate ingested during prolonged endurance exercise. Sports Medicine 14 (1): 27-42.

Hellsten-Westing, Y., Sollevi, A., Sjodin, B. (1991): Plasma accumulation of hypoxanthine, uric acid and creatine kinase following exhausting runs of differing durations in man. Eur. J. Appl. Physiol. 62: 380-384.

Henrikssen, E.J., Bourey, R.E., Rodnick, Koranyi,L., Permutt, M.A. and Holloszy, J.O. (1990): Glucose transporter protein content and glucose 
transport capacity in rat skeletal muscle. Am. J. Physiol. 259 (Endocrinol. Metab. 22): E593-E598.

Herbert, W.J. (1983): Water and Electrolytes. In: Ergogenic Aids in Sport, edited by Williams, M.H. Human Kinetics Publishers. Champaign, IL 61820.

Hermansen, L., Hultman, E., Saltin, B. (1967): Muscle glycogen during prolonged severe exercise. Acta Physiol. Scand. 71: 129-139.

Hickson, R.C., Rennie, M.J., Conlee, R.K., Winder, W.W. and Holloszy, J.O. (1977): Effects of increased plasma fatty acids on glycogen utilization and endurance. J. Appl. Physiol. 43 (5): 829-833.

Houmard, J.A., Egan, P.C., Johns, R.A., Neufer, P.D., Chenier, T.C., Israel, R.G. (1991): Gastric emptying during $1 \mathrm{~h}$ of cycling and running at $75 \% \mathrm{VO} 2$ max. Med. Sci. Sports Exerc. 23(3): 320-325.

Hultman, E. and Nilsson, L.H. (1971): Liver glycogen in man. Effect of different diets and muscular exercise. In Pernow, B. and Saltin, B. (eds): Muscle metabolism during exercise. Plenum Press, New York, pp. 143-151.

Hultman, E., Spriet, L.L. and Södelund, K. (1987): Energy metabolism and fatigue in working muscle. In: Exercise, benefits, limits and adaptations, edited by MacLeod, D., Maughan, R., Nimmo, M., Reilly, T. and Williams, C. E. \& F. N. SPON. London. New York.

Hultman, E. and Harris, R.C. (1988): Carbohydrate metabolism. In: Poortmans, J.R. (ed.): Principles of exercise Biochemistry. Med. Sport Sci. Basel, Karger, 27: 78-119.

Hunt, J.N. and Stubbs, D.F. (1975): The volume and energy content of meals as a determinant of gastric emptying. J. Physiol. (London) 245: 209215.

Hunt, J.B., Elliott, E.J., Fairclough, P.D. and Farthing, M.J.G. (1988): Effects of [Na] on water and $\mathrm{Na}$ absorption from hypotonic oral rehydration solutions (ORS). Clin. Sci. 74 (Suppl. 18): 2P. 
Ivy, J.L., Costill, D.L., Fink, W.J., and Lower, R.W. (1979): Influence of caffeine and carbohydrate feedings on endurance performance. Med. Sci. Sports, 11: 6-11.

Ivy, J.L., Miller, W., Dover, V., Goodyear, L.G., Sherman, W.M., Farrell, S. and Williams, H. (1983): Endurance improved by ingestion of a glucose polymer supplement. Med. Sci. Sports. Exerc. 15(6): 466-471.

Jansson, E. (1981): Acid soluble and insoluble glycogen in human skeletal muscle. Acta Physiol. Scand. 113: 337-340.

Jansson, E., Hjemdahl, P. and Kaijser, L. (1986): Epinephrine-induced changes in muscle carbohydrate metabolism during exercise in male subjects. J. Appl. Physiol. 60: 1466-1470.

Jeukendrup, A.E. (1993): Exogenous carbohydrate oxidation from soluble and insoluble carbohydrate sources. Int. J. Sports Med. 14 (5): 296.

Jones, B.M.J., Brown, B.E., Loran, J.S., Edgerton, D., Kennedy, J.F., Stead, J.A. and Silk, D.B.A. (1983): Glucose absorption from starch hydrolysates in the human jejunum. Gut, 24: 1152-1160.

Jones, B.M.J., Higgins, B.E. and Silk, D.B.A. (1987): Glucose absorption from maltotriose and glucose oligomers in the human jejunum. Clinical Science, 72: 409-414.

Jones, D.A. and Round, J.M. (1992): Skeletal muscle in health and disease. A textbook of muscle physiology. Manchester University Press. Manchester and New York.

Kaijser, L. (1992): Heart muscle metabolism and function during heavy exercise. In: Marconnet, P., Komi, P.V., Saltin, B. and Sejersted, O.M. (Eds). Muscle fatigue mechanisms in exercise and training. Medicine and Sport Science, Vol. 34: 182-194.

Karlsson, J. and Saltin, B. (1971): Diet, muscle glycogen, and endurance performance. J.Appl. Physiol. 31(2): 203-206. 
Katz, A., Sahlin, K. and Broberg, S. (1991): Regulation of glucose utilization in human skeletal muscle during moderate dynamic exercise. Am. J. Physiol. 260 (23): E411-E415.

Katz, A., Broberg, S., Sahlin, K. and Wahren, J. (1986): Leg glucose uptake during maximal dynamic exercise in humans. Am. J. Physiol. 251 (14): E65E70.

Kingwell, B., McKenna, M.J., Sandstrom, E.R. and Hargreaves, M. (1989): Effect of glucose polymer ingestion on energy and fluid balance during exercise. J. Sports Sci. 7: 3-8.

Kjaer, M., Christensen, N.J., Sonne, B., Richter, E.A. and Galbo, H. (1985): The effect of exercise on epinephrine turnover in trained and untrained man. J. Appl. Physiol. 59: 1061-1067.

Kjaer, M., Engfred, K., Fernandes, A., Secher, N.H. and Galbo, H. (1990): Influence of sympathoadrenergic activity on hepatic glucose production during exercise in man. Med. Sci. Sports Exerc. 22 (Suppl. 2): S54.

Krzentowski, G., Pirnay, F., Luyckx, A.S., Lacroix, M., Mosora, F. and LeFebvre, P.J. (1984): Effect of physical training on utilization of a glucose load given orally during exercise. Am. J. Physiol. 246: E412-E417.

Kuipers, H., Keizer, H.A., Brouns, F., Sarris, W.H.M. (1987): Carbohydrate feeding and glycogen synthesis during exercise in man. Pflu. Arch. 410: 652-656.

Lamb, D.R., Brodowicz, G.R. (1986): Optimal use of fluids of varying formulations to minimise exercise-induced disturbances in homeostasis. Sports med. 3: 247-274.

Laurell, S. and Tibbling, G. (1966): An enzymatic fluorometric micromethod for the determination of glycerol. Clin. Chim. Acta 13: 312316.

Leiper, J.B. and Maughan, R.J. (1986a): The effect of luminal tonicity on 
water absorption from a segment of the intact human jejunum. J. Physiol. 378: $95 \mathrm{P}$.

Leiper, J.B. and Maughan, R.J. (1986b): Absorption of water and electrolytes from hypotonic, isotonic and hypertonic solutions. J. Physiol. 373: 90P.

Lowry, O.H. and Passonneau, J.V. (1972): A flexible system of enzymatic analysis, 189-193, Academic Press, New York.

Macaraeg, P.V.J. (1983): Influence of carbohydrate electrolyte ingestion on running endurance. In: Fox, E.L. (Ed). Nutrient utilization during exercise. Ross Laboratories, Columbus, 91-96

MacLean, D.A., Spriet, L.L. and Graham,T.E. (1992): Plasma amino acid and ammonia responses to altered dietary intakes prior to prolonged exercise in humans. Can. J. Physiol. Pharmacol. 70: 420-427.

Madsen, K., Pedersen, P.K., Rose, P. and Richter, E.A. (1990): Carbohydrate supercompensation and muscle glycogen utilization during exhaustive running in highly trained athletes. Eur. J. Appl. Physiol. 61: 467-472.

Martin III, W.H., Coggan, A.R., Spina, R.J. and Saffitz, J.E. (1989): Effect of fiber type and training on beta-adrenoceptor density in human skeletal muscles. Am. J. Physiol. 257: E736-E742.

Massicotte, D., Peronnet, F., Allah, C., Hillaire-Marcel, C., Ledoux, M. and Brisson, G.(1986): Metabolic response to [13 C]glucose and [ $\left.{ }^{13} \mathrm{C}\right]$ fructose ingestion during exercise. J. Appl. Physiol. 61(3): 1180-1184.

Massicotte, D., Peronnet, F., Brisson, G., Bakkouch, K. and Hillaire-Marcel, C. (1989): Oxidation of a glucose polymer during exercise. J. Appl. Physiol. 66(1): 179-183.

Maughan, R.J. (1982): A simple rapid method for the determination of glucose, lactate, pyruvate, alanine, 3-hyroxybutyrate and acetoacetate on a single $20 \mu \mathrm{l}$ blood sample. Clin. Chem. Acta 122: 231-240. 
Maughan, R.J., Leiper, J.B., Thompson, J. (1985): Rectal temperature after marathon running. Br. J. Sports Med. 19: 192-196.

Maughan, R.J., Fenn, C.E., Gleeson, M. and Leiper, J.B. (1987): Metabolic and circulatory responses to the ingestion of glucose polymer and glucose/electrolyte solutions during exercise in man. Eur. J. Appl. Physiol. 56: 356-362.

Maughan, R.J., Fenn, C.E., Leiper, J.B. (1989): Effects of fluid, electrolyte and substrate ingestion on endurance capacity. Eur. J. Appl. Physiol. 58: 481-486.

Maughan, R.J., Leiper, J.B. and McGaw, B.A. (1990): Effects of exercise intensity on absorption of ingested fluids in man. Exp. Physiol. 75: 419-421.

Maughan, R.J. (1991): Fluid and electrolyte loss and replacement in exercise. J. Sports Sciences, 9: 117-142.

Miles, P.D.G., Finegood, D.T., Lavina, H., Lickley, A. and Vranic, M. (1992): Regulation of glucose turnover at the onset of exercise in the dog. J. Appl. Physiol. 72 (6): 2487-2494.

Millard-Stafford, M.L., Sparling, P.B., Rosskopf, L.B. and Dicarlo, L.J. (1992): Carbohydrate-electrolyte replacement improves distance running performance in the heat. Med. Sci. Sports Exerc. 24 (8): 934-940.

Mitchell, J.B. Costill, D.L., Houmard, J.A., Flynn, M.G., Fink, W.J. and Beltz, J.D. (1988): Effects of carbohydrate ingestion on gastric emptying and exercise performance. Med. Sci. Sports Exerc., 20(2): 110-115.

Mitchell, J.B., Costill, D.L, Houmard, J.A., Fink, W.J., Pascoe, D.D., and Pearson, D.R. (1989a): Influence of carbohydrate dosage on exercise performance and glycogen metabolism. J. Appl. Physiol. 67(5): 1843-1849.

Mitchell, J.B., Costill, D.L., Houmard, J.A., Fink, W.J., Robergs, R.A., Davis, J.A. (1989b): Gastric emptying: influence of prolonged exercise and carbohydrate concentration. Med. Sci. Sports Exerc. 21: 269-274. 
Mitchell, J.B. and Voss, K.W. (1991): The influence of volume on gastric emptying and fluid balance during prolonged exercise. Med. Sci. Sports Exerc. 23 (3): 314-319.

Montain, S.J. and Coyle, E.F. (1992): Fluid ingestion during exercise increases skin blood flow independent of increases in blood volume. J. Appl. Physiol. 73 (3): 903-910.

Murray, R., Paul, G.L., Seifert, J.G., Eddy, D.E. (1991): Responses to varying rates of carbohydrate ingestion during exercise. Med. Sci. Sports Exerc. 23 (6): 713-718.

Murray, R. (1987): The effects of consuming carbohydrate-electrolyte beverages on gastric emptying and fluid absorption during and following exercise. Sports Med. 4: 322-351.

Myhre, L.G., Hartung, G.H.; Nunneley, S.A., Tucker, D.M. (1985): Plasma volume changes in middle-aged male and female subjects during marathon running. J. Appl. Physiol. 59: 559-563.

Naveri, H., Tikkanen, H., Kairento, A.L. and Harkonen, M. (1989): Gastric emptying and serum insulin levels after intake of glucose-polymer solutions. Eur. J. Appl. Physiol. 58: 661-665.

Nesher, R., Karl, I.E. and Kipnis, D.M. (1985): Dissociation of effects of insulin and contraction on glucose transport in rat epitrochlearis muscle. Am. J. Physiol. 249: C226-C232.

Neufer, P.D., Costill, D.L., Fink, W.J., Kirwan, P., Field, R.A. and Flynn, M.G. (1986): Effects of exercise and carbohydrate composition on gastric emptying. Med. Sci. Sports Exerc. 18(6): 658-662.

Neufer, P.D., Young, A.J. and Sawka, M.N. (1989): Gastric emptying during walking and running : effects of varied exercise intensity. Eur. J. Appl. Physiol. 58: 440-445.

Newsholme, E.A. and Start, C. (1973): Regulation in metabolism. WileyInterscience, London. 
Newsholme, E.A., Acworth, I.N. and Blomstrand, E. (1987): Amino acids, brain neurotransmitters and a functional link between muscle and brain that is important in sustained exercise. In: Benzi, G. (Ed): Advances in myochemistry. John Libby Eurotext, London, 127-138.

Nielsen, B., Savard, G., Richter, E.A., Hargreaves, M. and Saltin, B. (1990): Muscle blood flow and muscle metabolism during exercise and heat stress. J. Appl. Physiol. 69 (3): 1040-1046.

Nilsson, L.H. and Hultman, E. (1973): Liver glycogen in man - the effect of total starvation or a carbohydrate-poor diet followed by carbohydrate refeeding. Scand. J. Clin. Lab. Invest. 32: 325-330.

Norman, B., Sollevi, A. and Jansson, E. (1988): Increased IMP content in glycogen-depleted muscle fibres during submaximal exercise in man. Acta Physiol. Scand. 133: 97-100.

Noakes, T.D. (1987): Effect of exercise on serum enzyme activities in humans. Sports Medicine 4: 245-267.

Noakes, T.D., Adams, B.A., Myburgh, K.H., Greef, C., Lotz, T., Nathan, M. (1988): The danger of an inadequate water intake during prolonged exercise. Eur. J. Appl. Physiol. 57: 210-219.

Noakes, T.D., Myburgh, K.H., Plessis, J.D., Lang, L., Lambert, M., Van Der Riet, C., Schall, R. (1991a): Metabolic rate, not percent dehydration, predicts rectal temperature in marathon runners. Med. Sci. Sports Exerc. 23 (4): 443449.

Noakes, T.D., Rehrer, N.J., and Maughan, R.J. (1991b): The importance of volume in regulating gastric emptying. Med. Sci. Sports Exerc. 23 (3): 307313.

Owen, M.D., Kregel, K.C., Wall, P.T. and Gisolfi, C.V. (1986): Effects of ingesting carbohydrate beverages during exercise in the heat. Med. Sci. Sports Exerc. 18(5): 568-575. 
Pallikarakis, N., Jandrain, B., Pirnay, F., Mosora, F., Lacroix, M., Luyckx, A.S. and Lefebvre, P.J. (1986): Remarkable metabolic availability of oral glucose during long-duration exercise in humans. J. Appl. Physiol. 60(3): 1035-1042.

Pappenheimer, J.R. (1990): Paracellular intestinal absorption of glucose, creatinine, and mannitol in normal animals: relation to body size. Am. J. Physiol. 259 (22): G290-G299.

Paul, A.A., Southgate, D.A.T. (1978): McCance and Widdowson; the composition of foods. HMSO, London.

Pernow, B. and Saltin, B. (1971): Availability of substrates and capacity for prolonged heavy exercise in man. J. Appl. Physiol. 31: 416-422.

Peronnet, F., Massicotte, D., Brisson, G. and Hillaire-Marcel, C. (1990): Use of $13 \mathrm{C}$ substrates for metabolic studies in exercise: methodological considerations. J. Appl. Physiol. 69 (3): 1047-1052.

Peronnet, F., Adopo, E. and Massicotte, D. (1992): Exogenous substrate utilization during prolonged exercise: studies with $13 \mathrm{C}$-labeling. In: Marconnet, P., Komi, P.V., Saltin, B. and Sejersted, O.M. (Eds). Muscle fatigue mechanisms in exercise and training. Medicine and Sport Science, Vol. 34: 195-206.

Peronnet, F., Adopo, E. and Massicotte, D., Brisson, G. and Hillaire-Marcel, C. (1993): Comparison of two methods for computing exogenous substrate oxidation using ${ }^{13} \mathrm{C}$-labeling. Med. Sci. Sports Exerc. 25 (2): 297-302.

Pirnay, F., Lacroix, M., Mosora, F., Luyckx, A. and Lefebvre, P. (1977a): Glucose oxidation during prolonged exercise evaluated with naturally labelled 13Cglucose. J. Appl. Physiol. 43(2): 258-261.

Pirnay, F., Lacroix, M., Mosora, F., Luyckx, A. and Lefebvre, P. (1977b): Effect of glucose ingestion on energy substrate utilisation during prolonged muscular exercise. Eur. J. Appl. Physiol. 36, 247-254. 
Pirnay, F., Crielaard, J.M., Pallikarakis, N., Lacroix, M., Mosora, F., Krzentowski, G., Luyckx, A.S. and Lefebvre, P. (1982): Fate of exogenous glucose during exercise of different intensities in humans. J. Appl. Physiol. 53(6): 1620-1624.

Ploug, T., Galbo, H. and Richter, E.A. (1984): Increased muscle glucose uptake during contractions: no need for insulin. Am. J. Physiol. 247: E726E731.

Pugh, L.G.C.E., Corbett, J.L., Johnson, R.H. (1967): Rectal temperatures, weight losses, and sweat rates in marathon running. J. Appl. Physiol. 23 (3): 347-352.

Rehrer, N.J., Beckers, E., Brouns, F., Ten Hoor, F. and Saris W.H.M. (1989): Exercise and training effects on gastric emptying of carbohydrate beverages. Med. Sci. Sports Exerc., 21(5): 540-549.

Rehrer, N.J., Brouns, F., Beckers, E., Ten Hoor, F., and Saris, W.H.M. (1990): Gastric emptying with repeated drinking during running and bicycling. Int. J. Sports Med. 11:238-243.

Rehrer, N.J., Wagenmakers, A.J.M., Beckers, E.J., Halliday, D., Leiper, J.B., Brouns, F., Maughan, R.J., Westerterp, K. and Saris, W.H. (1992): Gastric emptying, absorption, and carbohydrate oxidation during prolonged exercise. J. Appl. Physiol. 72 (2): 468-475.

Ren, J.M. and Hultman, E. (1989): Regulation of glycogenolysis in human skeletal muscle. J. Appl. Physiol. 67 (6): 2243-2248.

Richter, E.A., Ruderman, N.B., Gavras, H. Belur, E.R. and Galbo, H. (1982): Muscle glycogenolysis during exercise: dual control by epinephrine and contractions. Am. J. Physiol. 242 (Endocrinol. Metab. 5): E25-E32.

Richter, E.A. and Galbo, H. (1986): High glycogen levels enhance glycogen breakdown in isolated contracting skeletal muscle. J. Appl. Physiol., 61: 827-831. 
Richter, E.A., Kiens, B., Saltin, B., Christensen, N.J. and Savard, G. (1988): Skeletal muscle glucose uptake during dynamic exercise in humans: role of muscle mass. Am. J. Physiol. 254 (17): E555-E561.

Riley, M.L., Israel, R.G., Holbert, D., Tapscott, E.B. and Dohm, G.L. (1988): Effect of carbohydrate ingestion on exercise endurance and metabolism after a 1-day fast. Int. J. Sports Med. 9: 320-324.

Rogers, M.A., Stull, G.A., Apple, F.S. (1985): Creatine kinase isoenzyme activities in men and women following a marathon race. Med. Sci. Sports Exerc. 17 (6): 679-682.

Romijn, J.A., Coyle, E.F., Sidossis, L., Gastaldelli, A. Horowitz, J.F., Endert, E. and Wolfe, R.R. (1993): Regulation of endogenous fat and carbohydrate metabolism in relation to exercise intensity and duration. Am. J. Physiol. 265 (28): E380-E391.

Ryan, A.J., Bleiler, T.L., Carter, J.E. and Gisolfi, C.V. (1989): Gastric emptying during prolonged cycling exercise in the heat. Med. Sci. Sports Exerc. 21 (1): 51-58.

Sahlin, K., Katz, A. and Broberg, S. (1990): Tricarboxylic acid cycle intermediates in human muscle during prolonged exercise. Am. J. Physiol. 259 (Cell Physiol. 28): C834-C841.

Saltin, B. and Karlsson, J. (1971): Muscle glycogen utilization during work of different intensities. In: Muscle metabolism during exercise, edited by Pernow, B. and Saltin, B. Plenum Press, New York.

Saltin, B., Gagge, A.P., Bergh, V. and Stolwijk, J.A.J. (1972): Body temperatures and sweating during exhaustive exercise. J. Appl. Physiol., 32: 635-643.

Saltin, B., Bangsbo, J., Graham, T.E., Johansen, L. (1992): Metabolism and performance in exhaustive intense exercise; different effects of muscle glycogen availability, previous exercise and muscle acidity. In: Marconnet, P., Komi, P.V., Saltin, B. and Sejersted, O.M. (Eds). Muscle fatigue 
mechanisms in exercise and training. Medicine and Sport Science, Vol. 34: 87-114.

Sasaki, H., Maeda, J., Usui, S. and Ishiko, T. (1987a): Effect of sucrose and caffeine ingestion on performance of prolonged strenuous running. Int. J. Sports Med. 8(4): 261-265.

Sasaki, H., Takaoka, I. and Ishiko, T. (1987b): Effects of sucrose or caffeine ingestion on running performance and biochemical responses to endurance running. Int. J. Sports Med. 8(3): 203-207.

Sawka, M.N., Young, A.J., Francesconi, R.P., Muza, S.R. and Pandolf, K.B. (1985): Thermoregulatory and blood responses during exercise at graded hypohydration levels. J. Appl. Physiol. 59: 1394-1401.

Sawka, M.N. (1992): Physiological consequences of hypohydration: exercise performance and thermoregulation. Med. Sci. Sports Exerc. 24 (6): 657-670.

Seidman, D.S., Ashkenazi, I., Arnon, R., Shapiro, Y. and Epstein, Y. (1991): The effect of glucose polymer beverage ingestion during prolonged outdoor exercise in the heat. Med. Sci. Sports Exerc. 23 (4): 458-462.

Sherman, W.M., Costill, D.L., Fink, W.J., Miller, J.M. (1981): Effect of exercise and diet manipulation on muscle glycogen and its subsequent utilization during performance. Int. J. Sports Med. 2: 114-118.

Sherman, W.M., Costill, D.L., Fink, W.J., Hagerman, F.C., Armstrong, L.E., Murray, T.F. (1983): Effect of $42.2 \mathrm{Km}$ footrace and subsequent rest or exercise on muscle glycogen and enzymes. J. Appl. Physiol., 55(4): 12191224.

Sjogaard, G. (1986): Water and electrolyte fluxes during exercise and their relation to muscle fatigue. Acta Physiol. Scand. 128 (Suppl. 556): 129-136.

Sjogaard, G., Adams, R.P. and Saltin, B. (1985): Water and ion shifts in skeletal muscle of humans with intense dynamic knee extension. Am. J. Physiol. 248 (17): R190-R196. 
Slentz, C.A., Davis, J.M., Settles, D.L., Pate, R.R. and Settles, S.J. (1990): Glucose feedings and exercise in rats: glycogen use, hormone responses, and performance. J. Appl. Physiol. 69 (3): 989-994.

Soeldner, J. and Sloane, D. (1965): Critical variables in the radioimmunoassay of serum insulin using the double antibody technique. Diabetes 14: 771-779.

Sole, C.C. and Noakes, T.D. (1989): Faster gastric emptying for glucosepolymer and fructose solutions than for glucose in humans. Eur. J. Appl. Physiol. 58: 605-612.

Spencer, M.K., Yan, Z. and Katz, A. (1991): Carbohydrate supplementation attenuates IMP accumulation in human muscle during prolonged exercise. Am. J. Physiol. 261 (Cell Physiol. 30): C71-C76.

Spriet, L.L., MacLean, D.A., Dyck, D.J., Hultman, E., Cederblad, G. and Graham, T.E. (1992): Caffeine ingestion and muscle metabolism during prolonged exercise in humans. Am. J. Physiol. 262 (Endocrinol. Metab. 25): E891-E898.

Stanley, W.C. and Connett, R.J. (1991): Regulation of muscle carbohydrate metabolism during exercise. FASEB J. 5: 2155-2159.

Tabata, I., Ogita, F., Miyachi, M. and Shibayama, H. (1991): Effect of low blood glucose on plasma CRF, ACTH, and cortisol during prolonged physical exercise. J. Appl. Physiol. 71 (5): 1807-1812.

Taylor, H. R., Buskirk, E. R., Henschell, A. (1955): Maximum oxygen uptake as an objective measure of cardio-respiratory performance. J. Appl. Physiol. 8: 73-80.

Tsintzas, O. K., Liu, R., Williams, C., Gaitanos, G.C., and Campbell, I.G. (1993): The effect of carbohydrate ingestion during exercise on a $30-\mathrm{km}$ race. Int. J. Sport Nutr. 3 (2): 127-139.

Van Handel, P.J., Fink, W.J., Branan, G., Costill, D.L. (1980): Fate of ${ }^{14} \mathrm{C}$ glucose ingested during prolonged exercise. Int. J. Sports Med. 1: 127-131. 
Vissing, J., Sonne, B. and Galbo, H. (1988): Regulation of hepatic glucose production in running rats studied by glucose infusion. J. Appl. Physiol. 65(6): 2552-2557.

Vist, G.E. and Maughan, R.J. (1993): Factors affecting the rate of gastric emptying in man. Inter. J. Sports Med. 14 (5): 298.

Vollestad, N.K., Vaage, O. and Hermansen, L. (1984): Muscle glycogen depletion patterns in type I and subgroups of Type II fibres during prolonged severe exercise in man. Acta Physiol. Scand. 122: 433-441.

Wagenmakers, A.J.M., Coakley, J.H. and Edwards, R.H.T. (1990): Metabolism of branched-chain amino acids and ammonia during exercise: clues from McArdle's disease. Int. J. Sports Med. 11: S101-S113.

Wagenmakers, A.J.M., Beckers, E.J., Brouns, F., Kuipers, H., Soeters, P.B., Van Der Vusse, G.J. and Sarris, W.H.M. (1991): Carbohydrate supplementation, glycogen depletion, and amino acid metabolism during exercise. Am. J. Physiol. 260 (23): E883-E890.

Wahren, J., Felig, P., Ahlborg, G. and Jorfeldt, L. (1971): Glucose metabolism during leg exercise in man. J. Clin. Invest. 50: 2715-2725.

Wasserman, D.H. Lickley, H.L. and Vranic, M. (1984): Interactions between glucagon and other counterregulatory hormones during normoglycemic and hypoglycemic exercise. J. Clin. Invest. 74: 1404-1413.

Wasserman, D.H., Spalding, J.A., Brooks Lacy, D., Colburn, C.A., Goldstein, R.E. and Cherrington, A.D. (1989): Glucagon is a primary controller of hepatic glycogenolysis and gluconeogenesis during muscular work. Am. J. Physiol. 257 (20): E108-E117.

Wasserman, D.H., Williams, P.E., Brooks Lacy, D., Bracy, D. and Cherrington, A.D. (1990): Hepatic nerves are not essential to the increase in hepatic glucose production during muscular work. Am. J. Physiol. 259 (22): E195-E203. 
Wells, C.L., Schrader, T.A., Stern, J.R. and Krahenbuhl, G.S. (1985): Physiological responses to a 20-mile run under three fluid replacement treatments. Med. Sci. Sports Exerc. 17 (3): 364-369.

Werner, W., Rey, H., Wielinger, H. (1970): Z. Analyt. Chem. 252: 224-228.

Wheeler, K.B. and Banwell, J.G. (1986): Intestinal water and electrolyte flux of glucose-polymer electrolyte solutions. Med. Sci. Sports Exerc. 18 (4): 436439.

Williams, C. and Nute, M.G. (1983): Some physiological demands of a half marathon race in recreational runners. Br. J. Sports Med. 17: 152-161.

Williams, C., Nute, M.G. and Walker, M.P. (1988): Influence of carbohydrate supplementation on running performance. Proc. Nutr. Soc.

Williams, C., Nute, M.G., Broadbank, L., Vinall, S. (1990): Influence of fluid intake on endurance running performance. Eur. J. Appl. Physiol. 60: 112-119.

Williams, C., Brewer, J. and Walker, M. (1992): The effect of a high carbohydrate diet on running performance during a $30-\mathrm{km}$ treadmill time trial. Eur. J. Appl. Physiol. 65: 18-24.

Williams, R.S. (1986): Adrenergic receptors of skeletal muscle. In: Biochemistry of Exercise VI. Ed. Saltin, B. Human Kinetics Publishers, Champaign, Illinois, 77-86.

Wright, D.A., Sherman, W.M., Dernbach, A.R. (1991): Carbohydrate feedings before, during, or in combination improve cycling endurance performance. J. Appl. Physiol. 71 (3): 1082-1088.

Wilber, R. L. and Moffat, R. J. (1992): Influence of carbohydrate ingestion on blood glucose and performance in runners. Int. J. Sport Nutr. 2: 317-327.

Young, S.N. (1986): The clinical psychopharmacology of tryptophan. In: Wurtman, R.J., Wurtman, J.J. (Eds). Nutrition and the brain, vol. 7. Raven, NY, 49-88. 


\section{APPENDIX A}

\section{MIXED MUSCLE METABOLITE ASSAYS}

\section{1/ Adenosine triphosphate and Phosphocreatine}

Principle:

$\mathrm{PCr}+\mathrm{ADP} \_\mathrm{CPK} \longrightarrow$ Creatine + ATP

ATP + Glucose __HK — $\longrightarrow$ ADP + G6P

$\mathrm{G} 6 \mathrm{P}+\mathrm{NADP}+{ }_{-} \mathrm{G} 6 \mathrm{P}-\mathrm{DH} \longrightarrow$ 6-P-Gluconolactone + NADPH $+\mathrm{H}^{+}$

\section{Reagents:}

Buffer: TRIS- $\mathrm{HCl} 50 \mathrm{mmol} / \mathrm{l}, \mathrm{BSA} 0.02 \%$, pH 8.1

Cofactor: NADP $5 \mathrm{mmol} / \mathrm{l}$

Enzymes: CK $1260 \mathrm{U} / \mathrm{ml}$; HK $28 \mathrm{U} / \mathrm{ml}$; G6P-DH $14 \mathrm{U} / \mathrm{ml}$

Reagents: ADP $10 \mathrm{mmol} / \mathrm{l}$; Glucose $10 \mathrm{mmol} / \mathrm{l}$; Dithiothreitol $50 \mathrm{mmol} / \mathrm{l}$; $\mathrm{MgCl}_{2} 0.1 \mathrm{~mol} / 1$

Standard: ATP $2 \mathrm{mmol} / \mathrm{l}$ (stock solution); $\mathrm{PCr} 2 \mathrm{mmol} / \mathrm{l}$

Additional reagents: Carbonate buffer $20 \mathrm{mmol} / \mathrm{l}, \mathrm{pH}$ 10.0; TRIS- $\mathrm{HCl} 20$ $\mathrm{mmol} / \mathrm{l}, \mathrm{BSA} 0.02 \%, \mathrm{pH} 8.1$ (diluent for enzyme solutions)

Working standards were prepared daily from the stock solution as follows:

stock solution $(\mu \mathrm{l})$

water $(\mu \mathrm{l})$

ATP concentration ( $\mu \mathrm{mol} / \mathrm{l})$

$\mathrm{PCr}$ concentration $(\mu \mathrm{mol} / \mathrm{l})$

$\begin{array}{rrrrr}0 & 25 & 50 & 100 & 200 \\ 2000 & 1975 & 1950 & 1900 & 1800 \\ 0 & 25 & 50 & 100 & 200 \\ & & & & 200\end{array}$

Immediately prior to analysis sufficient reaction mixture for 3 sets of duplicate blanks, standards and samples was prepared as follows: 


\section{Reaction mixture (final concentrations):}

Buffer 1ml; NADP $10 \mu \mathrm{l}(50 \mu \mathrm{mol} / \mathrm{l}) ;$ ADP $10 \mu \mathrm{l}(0.1 \mathrm{mmol} / \mathrm{l})$; Glucose $10 \mu \mathrm{l}$ (0.1 mmol/l); Dithiothreitol $10 \mu \mathrm{l}(0.5 \mathrm{mmol} / \mathrm{l}) ; \mathrm{MgCl}_{2} 50 \mu \mathrm{l}$ ( $\left.5 \mathrm{mmol} / \mathrm{l}\right)$; G6P$\mathrm{DH} 5 \mu \mathrm{l}(0.07 \mathrm{U} / \mathrm{ml})$

\section{Procedure:}

$1 / 20 \mu \mathrm{l}$ of extract was pipetted into a fluorimeter tube and diluted with $100 \mu \mathrm{I}$ of double-distilled water. Following this, $10 \mu \mathrm{l}$ of the diluted extract was transferred into 3 pair of tubes ( 3 sets of duplicate samples). Three sets of water blanks and 1 set of standards were also prepared using $10 \mu \mathrm{l}$ volumes.

$2 / 200 \mu \mathrm{l}$ of reaction mixture was added to one pair of tubes and blanks (G6P reagent)

$3 / 5 \mu \mathrm{l}$ of $\mathrm{HK}$ was added per $\mathrm{ml}$ of remaining reaction mixture. $200 \mu \mathrm{l}$ of this reaction mixture was then added to the second pair of tubes, blanks and standards (ATP reagent).

4/ $10 \mu \mathrm{l}$ of CK was added per ml of remaining reaction mixture. $200 \mu \mathrm{l}$ of this reaction mixture was then added to the third pair of tubes and blanks ( $\mathrm{PCr}$ reagent).

5/ A further $1 \mathrm{ml}$ of $\mathrm{PCr}$ reagent was added to $50 \mu \mathrm{l}$ of $0.2 \mathrm{mmol} / \mathrm{l} \mathrm{PCr}$ standard, and the reaction followed in the fluorimeter to confirm it was running, and to check the time course of incubation. After incubating for 30$40 \mathrm{~min}$ at room temperature, $1 \mathrm{ml}$ of carbonate buffer was added to each tube, and after thorough mixing fluorescence was measured. The $\mathrm{PCr}$ reagent gives the sum of PCr, ATP and G6P, the ATP reagent the sum of ATP and G6P, and the G6P reagent G6P on its own. The concentrations of ATP and $P C r$ were determined from the standard curve following the subtraction of G6P from $(A T P+G 6 P)$, and $(A T P+G 6 P)$ from $(P C r+A T P+G 6 P)$, respectively. The initial dilution factor was also taken into account. 


\section{2/Creatine}

Principle:

Creatine + ATP $\longrightarrow \mathrm{CK} \longrightarrow \mathrm{PCr}+\mathrm{ADP}$

$\mathrm{ADP}+\mathrm{PEP} \_\mathrm{PK} \longrightarrow \mathrm{ATP}+$ Pyruvate

Pyruvate $+\mathrm{NADH}+\mathrm{H}^{+} \_\mathrm{LDH} \longrightarrow$ Lactate $+\mathrm{NAD}^{+}$

\section{Reagents:}

Buffer: Low fluorescence imidazole, $50 \mathrm{mmol} / \mathrm{l}$, $\mathrm{pH} 7.5$

Cofactor: NADH $1 \mathrm{mmol} / 1$

Enzymes: CK $1260 \mathrm{U} / \mathrm{ml} ;$ PK $75 \mathrm{U} / \mathrm{ml} ; \mathrm{LDH} 240 \mathrm{U} / \mathrm{ml}$

Reagents: ATP $10 \mathrm{mmol} / \mathrm{l} ;$ PEP $2 \mathrm{mmol} / \mathrm{l} ; \mathrm{MgCl}_{2} 0.1 \mathrm{~mol} / \mathrm{l} ; \mathrm{KCl} 3 \mathrm{~mol} / \mathrm{l}$;

EDTA $0.1 \mathrm{~mol} / 1$

Standard: Creatine $2 \mathrm{mmol} / 1$ (stock solution)

Additional reagents: Carbonate buffer $20 \mathrm{mmol} / \mathrm{l}, \mathrm{pH}$ 10.0; TRIS- $\mathrm{HCl} 20$ $\mathrm{mmol} / 1, \mathrm{BSA} 0.02 \%, \mathrm{pH} 8.1$ (diluent for enzyme solutions)

Working standards were prepared daily from the stock solution as follows:

stock solution $(\mu \mathrm{l})$

water $(\mu \mathrm{l})$

creatine concentration $(\mu \mathrm{mol} / \mathrm{l})$

$\begin{array}{cccr}0 & 50 & 100 & 150 \\ 2000 & 1950 & 1900 & 1850 \\ 0 & 50 & 100 & 150\end{array}$

Immediately prior to the analysis sufficient reaction mixture for 2 sets of duplicate blanks, standards and samples was prepared as follows:

\section{Reaction mixture (final concentrations):}

Buffer $1 \mathrm{ml} ; \mathrm{MgCl}_{2} 50 \mu \mathrm{l}(5 \mathrm{mmol} / \mathrm{l}) ; \mathrm{KCl} 10 \mu \mathrm{l}(30 \mathrm{mmol} / \mathrm{l}) ;$ ATP $20 \mu \mathrm{l}(0.2$ $\mathrm{mmol} / \mathrm{l}) ;$ PEP $25 \mu \mathrm{l}(25 \mathrm{umol} / \mathrm{l}) ; \mathrm{NADH} 15 \mu \mathrm{l}(15 \mathrm{umol} / \mathrm{l}) ;$ EDTA $1 \mu \mathrm{l}(0.1$ $\mathrm{mmol} / \mathrm{l}) ; \mathrm{LDH} 2 \mu \mathrm{l}(0.48 \mathrm{U} / \mathrm{ml}) ;$ PK $10 \mu \mathrm{l}(0.75 \mathrm{U} / \mathrm{ml})$ 


\section{Procedure:}

$1 / 20 \mu \mathrm{l}$ of extract was pipetted into a fluorimeter tube and diluted with $100 \mu \mathrm{l}$ of double distilled water. Following this, $10 \mu \mathrm{l}$ of the diluted extract was transferred into 2 pair of tubes ( 2 sets of duplicate samples). Two sets of water blanks and 1 set of standards were also prepared using $10 \mu \mathrm{l}$ volumes.

$2 / 200 \mu \mathrm{l}$ of reaction mixture was added to one pair of tubes and blanks (ADP reagent)

$3 / 10 \mu \mathrm{l}$ of $\mathrm{CK}$ was added per $\mathrm{ml}$ of remaining reaction mixture. $200 \mu \mathrm{l}$ of this reaction mixture was then added to the second pair of tubes, blanks and standards (Creatine reagent).

4/ A further $1 \mathrm{ml}$ of the creatine reagent was added to $50 \mu \mathrm{l}$ of the $150 \mu \mathrm{mol} / \mathrm{l}$ creatine standard and the reaction in this tube followed on the chart recorder. After incubating for 60-65 min at room temperature, $1 \mathrm{ml}$ of carbonate buffer was added to each tube. Following thorough mixing fluorescence was measured. The Creatine reagent gives the sum of $\mathrm{Cr}, \mathrm{ADP}$ and pyruvate and the ADP reagent the sum of ADP and pyruvate. Concentrations of Creatine were determined from the standard curve following subtraction of (ADP+Pyruvate) from ( $\mathrm{Cr}+\mathrm{ADP}+\mathrm{Pyruvate})$. The initial dilution factor was also taken into account. 


\section{3) Lactate}

\section{Principle:}

Lactate $+\mathrm{NAD}^{+}-\mathrm{LDH} \longrightarrow$ Pyruvate $+\mathrm{NADH}+\mathrm{H}^{+}$

\section{Reagents:}

Buffer: Hydrazine $1.1 \mathrm{~mol} / \mathrm{l}$, pH 9.0 with $1 \mathrm{mmol} / \mathrm{l}$ EDTA

Cofactor: NAD $50 \mathrm{mmol} / 1$

Enzymes: LDH $5500 \mathrm{U} / \mathrm{ml}$ (undiluted)

Standard: Lactate $2 \mathrm{mmol} / \mathrm{l}$ (stock solution)

Additonal reagent: Carbonate buffer $20 \mathrm{mmol} / \mathrm{l}, \mathrm{pH} 10.0$

Working standards were prepared daily from the stock solution as follows:

Stock solution $(\mu \mathrm{l})$

Water $(\mu \mathrm{l})$

Lactate concentration $(\mu \mathrm{mol} / \mathrm{l})$

$\begin{array}{rrrr}0 & 50 & 100 & 150 \\ 2000 & 1950 & 1900 & 1850 \\ 0 & 50 & 100 & 150\end{array}$

Immediately prior to the analysis sufficient reaction mixture for 1 set of duplicate samples, water blanks and standards plus $1 \mathrm{ml}$ to follow the reaction on the chart recorder was prepared as follows:

Reaction mixture (final concentration):

Buffer $1 \mathrm{ml}$; NAD $10 \mu \mathrm{l}(0.5 \mathrm{mmol} / \mathrm{l})$; LDH $8 \mu \mathrm{l}(44 \mathrm{U} / \mathrm{ml})$

\section{Procedure:}

$1 / 10 \mu \mathrm{l}$ of undiluted extract was added to 1 set of duplicate samples, water blanks and standards

$2 / 0.2 \mathrm{ml}$ of reaction mixture was then added to each tube. 
3/ A further $1 \mathrm{ml}$ of reaction mixture was added to $50 \mu \mathrm{l}$ of $0.15 \mathrm{mmol} / \mathrm{l}$ lactate standard and the reaction in this tube followed on the chart recorder.

4/ After $30 \mathrm{~min}$ incubation, $1 \mathrm{ml}$ of carbonate buffer was added, the contents were mixed and fluorescence was read. Lactate concentration was calculated from the standard curve. 


\section{4) Glycogen}

The assay for glycogen was carried out on both the acid precipitated muscle pellet (acid insoluble glycogen) and the neutralised extract (acid soluble glycogen) by analysis of glucosyl units obtained after acid hydrolysis of glycogen by the method of Jansson (1981).

\section{Acid Hydrolysis:}

\section{Reagents:}

$\mathrm{HCl} 1 \mathrm{~mol} / 1 ; \mathrm{NaOH} 6 \mathrm{~mol} / \mathrm{l}$

\section{Procedure:}

For each muscle sample, $0.1 \mathrm{ml}$ of $1 \mathrm{~mol} / 1 \mathrm{HCl}$ per $1 \mathrm{mg}$ of muscle powder was added to the precipitated muscle pellet. $0.1 \mathrm{ml}$ of $1 \mathrm{~mol} / 1 \mathrm{HCl}$ was also added to $20 \mu \mathrm{l}$ of undiluted neutralised extract. Both samples were gently mixed and incubated for 2 hours in tightly sealed screw top micro-reaction (eppendorf) tubes in a boiling water bath. The tubes were then centrifuged for $1 \mathrm{~min}$ and left at room temperature to cool down. The acid hydrolysed extract only was neutralised with $15 \mu \mathrm{l}$ of $6 \mathrm{~mol} / \mathrm{l} \mathrm{NaOH}$.

Acid insoluble glycogen was assayed spectrophotometrically using a Glucose Test Combination, GOD/Perid method. Acid soluble glycogen was assayed fluorimetrically for glucose.

\section{Acid insoluble glycogen}

\section{Principle:}

glucose $+\mathrm{O}_{2}+\mathrm{H}_{2} \mathrm{O} \_\mathrm{GOD} \longrightarrow$ Gluconate $+\mathrm{H}_{2} \mathrm{O}_{2}$

$\mathrm{H}_{2} \mathrm{O}_{2}+\mathrm{ABTS} \longrightarrow \mathrm{POD} \longrightarrow$ coloured complex $+\mathrm{H}_{2} \mathrm{O}$

where:

$\mathrm{GOD}=$ glucose oxidase

$P O D=$ horseradish peroxidase

ABTS = di-ammonium 2,2'-azino-bis (3-ethylbenzothiazoline-6-sulphonate) 
The intensity of the colour change is directly proportional to the concentration of glucose. Therefore, the glucose concentration of a sample can be calculated by using a standard of known concentration.

\section{Reagents:}

Buffer/enzymes/chromogen: made up as directed in kit (phosphate buffer $0.1 \mathrm{~mol} / \mathrm{l}$, pH 7.0; POD $0.8 \mathrm{U} / \mathrm{ml}$; GOD $10 \mathrm{U} / \mathrm{ml}$; ABTS $1.0 \mathrm{mg} / \mathrm{ml}$ ). This was found to remain active for several months when stored protected from light at $0-4^{\circ} \mathrm{C}$.

Standard: Glucose $0.505 \mathrm{mmol} / \mathrm{l}$

\section{Procedure:}

Five blanks, five standards and duplicate samples were prepared by adding $10 \mu \mathrm{l}$ of double-distilled water, standard and the unneutralised hydrolysed extract of the precipitated muscle pellet, respectivelly, to $2 \mathrm{ml}$ of the GOD/Perid reagent. The samples were incubated at room temperature for 30 mins and their absorbance $(A)$ was then determined on a spectrophotometer at $436 \mathrm{~nm}$.

Acid insoluble glycogen concentration (glucosyl units $\mathrm{mmol} / \mathrm{kg} \mathrm{dm}$ ) was calculated using the following equation:

$$
\text { [Glycogen] }=(\text { A sample } / \text { A standard }) \times 0.505 \times 100
$$




\section{Acid soluble glycogen}

\section{Principle:}

Glucose + ATP _ HK $\longrightarrow$ G6P + ADP

$\mathrm{G} 6 \mathrm{P}+\mathrm{NADP}^{+} \_\mathrm{G} 6 \mathrm{P}-\mathrm{DH} \longrightarrow$ _-P-Gluconolactone + NADPH $+\mathrm{H}^{+}$

\section{Reagents:}

Buffer: TRIS-HCl $0.1 \mathrm{~mol} / \mathrm{l}, \mathrm{BSA} 0.02 \%, \mathrm{pH} 8.1$

Cofactor: NADP $5 \mathrm{mmol} / \mathrm{l}$

Enzymes: HK $28 \mathrm{U} / \mathrm{ml}$; G6P-DH $7 \mathrm{U} / \mathrm{ml}$

Reagents: ATP $50 \mathrm{mmol} / \mathrm{l} ; \mathrm{MgCl}_{2} 0.1 \mathrm{~mol} / \mathrm{l}$; EDTA $0.1 \mathrm{~mol} / \mathrm{l}$; Dithiothreitol $50 \mathrm{mmol} / \mathrm{l} ; \mathrm{HCl} 1 \mathrm{~mol} / \mathrm{l}$

Standard: Glucose $0.505 \mathrm{mmol} / \mathrm{l}$ (stock solution)

Additional reagents: Carbonate buffer $20 \mathrm{mmol} / \mathrm{l}, \mathrm{pH}$ 10.0; TRIS- $\mathrm{HCl} 20$ $\mathrm{mmol} / \mathrm{l}, \mathrm{BSA} 0.02 \%$, pH 8.1 (diluent for enzyme solutions).

Working standards were prepared daily from the stock solution as follows:

stock solution $(\mu \mathrm{l})$

water $(\mu \mathrm{l})$

glucose concentration $(\mu \mathrm{mol} / \mathrm{l}) \quad 0$

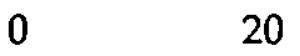

505

0

485

20

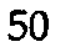

455

50
100

405

100

Immediately prior to the analysis sufficient reaction mixture for two sets of duplicate blanks, standards and samples was prepared as follows:

Reaction mixture (final concentrations):

Buffer $1 \mathrm{ml}$ : NADP $6 \mu \mathrm{l}(30 \mathrm{umol} / \mathrm{l})$; ATP $6 \mu \mathrm{l}(0.3 \mathrm{mmol} / \mathrm{l}) ; \mathrm{MgCl}_{2} 10 \mu \mathrm{l}(1$ $\mathrm{mmol} / \mathrm{l})$; EDTA $5 \mu \mathrm{l}(0.5 \mathrm{mmol} / \mathrm{l}) ;$ Dithiothreitol $10 \mu \mathrm{l}(0.5 \mathrm{mmol} / \mathrm{l}) ; \mathrm{G} 6 \mathrm{P}-\mathrm{DH}$ $3 \mu \mathrm{l}(0.02 \mathrm{U} / \mathrm{ml})$ 


\section{Procedure:}

1/ Two sets of $20 \mu \mathrm{l}$ of water blanks and samples (neutralised hydrolysed extract) and 1 set of standards were pipetted into fluorometric tubes.

$2 / 200 \mu \mathrm{l}$ of reaction mixture was added to one set of samples and blanks (G6P reagent)

$3 / 5 \mu \mathrm{l}$ of $\mathrm{HK}$ was added per $\mathrm{ml}$ of remaining reaction mixture. $200 \mu \mathrm{l}$ of this reaction mixture was then added to the second pair of samples, blanks and standards (Glucose reagent).

4/ A further $1 \mathrm{ml}$ of the Glucose reagent was added to $50 \mu \mathrm{l}$ of the $100 \mu \mathrm{mol} / 1$ glucose standard and the reaction in this tube followed on the chart recorder. Following a $30 \mathrm{~min}$ incubation at room temperature, $1 \mathrm{ml}$ of carbonate buffer was added to each tube, and after thorough mixing fluorescence was measured. Acid soluble glycogen concentration (as glucosyl units) was calculated from the the standard curve after subtracting G6P values from values obtained in step 3 above. The resulting value was multiplied by $(0.125 * 6.75)$ to correct for the dilution involved in the extraction and acid hydrolysis. Muscle free glucose was subtracted from the result to give the true acid-soluble glycogen concentration. This assay also enabled the concentration of G-6-P to be determined by using appropriate standards. 


\section{5/ Free glucose assay}

\section{Principle:}

Glucose + ATP _ HK $\longrightarrow$ G6P + ADP

$\mathrm{G} 6 \mathrm{P}+\mathrm{NADP}^{+} \_\mathrm{G} 6 \mathrm{P} \cdot \mathrm{DH} \longrightarrow$ _-P-Gluconolactone + $\mathrm{NADPH}+\mathrm{H}^{+}$

\section{Reagents:}

Buffer: TRIS-HCl $0.1 \mathrm{~mol} / \mathrm{l}, \mathrm{BSA} 0.02 \%, \mathrm{pH} 8.1$

Cofactor: NADP $5 \mathrm{mmol} / 1$

Enzymes: HK $28 \mathrm{U} / \mathrm{ml}$; G6P-DH $7 \mathrm{U} / \mathrm{ml}$

Reagents: ATP $50 \mathrm{mmol} / \mathrm{l} ; \mathrm{MgCl}_{2} 0.1 \mathrm{~mol} / \mathrm{l}$; EDTA $0.1 \mathrm{~mol} / \mathrm{l}$; Dithiothreitol $50 \mathrm{mmol} / \mathrm{l} ; \mathrm{HCl} 1 \mathrm{~mol} / \mathrm{l}$

Standard: Glucose $0.505 \mathrm{mmol} / \mathrm{l}$ (stock solution)

Additional reagents: Carbonate buffer $20 \mathrm{mmol} / \mathrm{l}, \mathrm{pH}$ 10.0; TRIS- $\mathrm{HCl} 20$ $\mathrm{mmol} / \mathrm{l}, \mathrm{BSA} 0.02 \%, \mathrm{pH} 8.1$ (diluent for enzyme solutions)

Working standards were prepared daily from the stock solution as follows:

$\begin{array}{lllcl}\text { stock solution }(\mu \mathrm{l}) & 0 & 20 & 50 & 100 \\ \text { water }(\mu \mathrm{l}) & 505 & 485 & 455 & 405 \\ \text { glucose concentration }(\mu \mathrm{mol} / \mathrm{l}) & 0 & 20 & 50 & 100\end{array}$

Immediately prior to the analysis sufficient reaction mixture for one set of duplicate blanks, standards and samples was prepared as follows:

\section{Reaction mixture (final concentrations):}

Buffer $1 \mathrm{ml}$ : NADP $6 \mu \mathrm{l}(30 \mathrm{umol} / \mathrm{l}) ;$ ATP $6 \mu \mathrm{l}(0.3 \mathrm{mmol} / \mathrm{l}) ; \mathrm{MgCl}_{2} 10 \mu \mathrm{l}(1$ mmol/l); EDTA $5 \mu \mathrm{l}(0.5 \mathrm{mmol} / \mathrm{l})$; Dithiothreitol $10 \mu \mathrm{l}(0.5 \mathrm{mmol} / \mathrm{l})$; G6P-DH $3 \mu \mathrm{l}(0.02 \mathrm{U} / \mathrm{ml})$; HK $5 \mu \mathrm{l}(0.14 \mathrm{U} / \mathrm{ml})$ 


\section{Procedure:}

$1 / 20 \mu \mathrm{l}$ of water blanks, samples (neutralised undiluted extract) and standards were pipetted into fluorometric tubes.

$2 / 200 \mu \mathrm{l}$ of reaction mixture was added to each tube.

3/ A further $1 \mathrm{ml}$ of the reaction mixture was added to $50 \mu \mathrm{l}$ of the $100 \mu \mathrm{mol} / \mathrm{l}$ glucose standard and the reaction in this tube followed on the chart recorder. Following a $30 \mathrm{~min}$ incubation at room temperature, $1 \mathrm{ml}$ of carbonate buffer was added to each tube, and after thorough mixing fluorescence was measured. Glucose concentration was calculated from the standard curve. G6P was subtracted from the result to give the true glucose concentration. 


\section{APPENDIX B}

\section{SPECTROPHOTOMETRIC ASSAYS FOR VALIDATION OF STANDARDS}

All assays were carried out in a $1 \mathrm{ml}$ semi-micro cuvette at $340 \mathrm{~nm}$, reading against air, and were followed to completion on a chart recorder. The concentration of the standard was calculated using the following equation:

$$
[C]=V c *\left(E_{2}-E_{1}\right) / 6.22 * V a
$$

where: $[\mathrm{C}]=$ the concentration of the standard $(\mathrm{mmol} / \mathrm{l})$

$\mathrm{Vc}=$ the final cuvette volume $(\mathrm{ml})$

$\mathrm{Va}=$ the volume of standard added (ml)

$E 1=$ the initial absorbance

$E 2$ = the final absorbance

6.22 = the extinction coefficient for NADH/NADPH at $340 \mathrm{~nm}$ 


\section{1/Adenosine triphosphate}

Principle:

ATP + Glucose __HK___ A ADP + G6P

$\mathrm{G} 6 \mathrm{P}+\mathrm{NADP}+$-_G6P-DH_— $\longrightarrow$ 6-P-Gluconolactone + NADPH $+\mathrm{H}^{+}$

\section{Reagents:}

Buffer: TRIS-HCl $50 \mathrm{mmol} / 1, \mathrm{BSA} 0.02 \%$, pH 8.1

Cofactor: NADP $50 \mathrm{mmol} / \mathrm{l}$

Enzymes: HK $28 \mathrm{U} / \mathrm{ml}$; G6P-DH $7 \mathrm{U} / \mathrm{ml}$

Other reagents: Glucose $0.1 \mathrm{~mol} / 1 ; \mathrm{MgCl}_{2} 0.1 \mathrm{~mol} / 1$

Standard: ATP $2 \mathrm{mmol} / 1$

\section{Reaction mixture (final concentrations):}

Buffer $1 \mathrm{ml}$; NADP $50 \mu \mathrm{l}(0.5 \mathrm{mmol} / \mathrm{l}) ; \mathrm{MgCl}_{2} 50 \mu \mathrm{l}(5 \mathrm{mmol} / \mathrm{l})$; Glucose 10 $\mu \mathrm{l}(1 \mathrm{mmol} / \mathrm{l}) ; \mathrm{G} 6 \mathrm{P}-\mathrm{DH} 10 \mu \mathrm{l}(0.07 \mathrm{U} / \mathrm{ml})$

\section{Procedure:}

$1 \mathrm{ml}$ of reaction mixture was added to $20 \mu \mathrm{l}$ of $2 \mathrm{mmol} / \mathrm{l}$ ATP standard. E1 was then read. $10 \mu \mathrm{l}$ of $\mathrm{HK}$ was then added and the reaction followed to completion ( $5 \mathrm{~min}$ ). E2 was then read. A further $10 \mu \mathrm{l}$ of $\mathrm{HK}$ was then added to determine the absorbance of the enzyme $\left(E_{H K}\right)$. The concentration of standard was calculated using the formula (1), where: $E=$ $\mathrm{E}_{2}-\mathrm{E}_{1}-\mathrm{E}_{\mathrm{HK}}$ 


\section{2/ Creatine}

\section{Principle:}

Creatine + ATP _

$\mathrm{ADP}+\mathrm{PEP}$ _- PK___

Pyruvate $+\mathrm{NADH}+\mathrm{H}^{+}-\mathrm{LDH} \longrightarrow$ Lactate $+\mathrm{NAD}^{+}$

\section{Reagents:}

Buffer: Low fluorescence imidazole, $50 \mathrm{mmol} / \mathrm{l}, \mathrm{pH} 7.5$

Cofactor: NADH $5 \mathrm{mmol} / \mathrm{l}$

Enzymes: CK $1500 \mathrm{U} / \mathrm{ml}$; PK $75 \mathrm{U} / \mathrm{ml}$; LDH $60 \mathrm{U} / \mathrm{ml}$

Reagents: ATP $10 \mathrm{mmol} / \mathrm{l} ;$ PEP $30 \mathrm{mmol} / \mathrm{l} ; \mathrm{MgCl}_{2} 0.1 \mathrm{~mol} / \mathrm{l} ; \mathrm{KCl} 3 \mathrm{~mol} / \mathrm{l}$

Standard: Creatine $2 \mathrm{mmol} / 1$

\section{Reaction mixture (final concentrations):}

Buffer $1 \mathrm{ml} ; \mathrm{MgCl}_{2} 50 \mu \mathrm{l}(5 \mathrm{mmol} / \mathrm{l}) ; \mathrm{KCl} 10 \mu \mathrm{l}(75 \mathrm{mmol} / \mathrm{l}) ; \mathrm{NADH} 15 \mu \mathrm{l}$ $(0.075 \mathrm{mmol} / \mathrm{l}) ;$ ATP $50 \mu \mathrm{l}(5 \mathrm{mmol} / \mathrm{l}) ;$ PEP $6.5 \mu \mathrm{l}(0.2 \mathrm{mmol} / \mathrm{l}) ;$ PK $10 \mu \mathrm{l}$ $(0.75 \mathrm{U} / \mathrm{ml}) ; \mathrm{LDH} 4 \mu \mathrm{l}(0.2 \mathrm{U} / \mathrm{ml})$

\section{Procedure:}

$1 \mathrm{ml}$ of reaction mixture was added to $20 \mu \mathrm{l}$ of standard. E1 was then read. $10 \mu \mathrm{l}$ of $\mathrm{CK}(15 \mathrm{U} / \mathrm{ml})$ was then added and the reaction followed to completion $(60 \mathrm{~min})$. E2 was then read. The concentration of standard was calculated using the formula (1). 


\section{3/ Glucose-6-phosphate}

Principle:

$\mathrm{G} 6 \mathrm{P}+\mathrm{NADP}^{+}+\mathrm{G} 6 \mathrm{P}-\mathrm{DH} \longrightarrow$ 6-P-Gluconolactone + $\mathrm{NADPH}+\mathrm{H}^{+}$

\section{Reagents:}

Buffer: TRIS- $\mathrm{HCl} 0.1 \mathrm{~mol} / \mathrm{l}, \mathrm{BSA} 0.02 \%$, pH 8.1

Cofactor: NADP $5 \mathrm{mmol} / 1$

Enzymes: G6P-DH $7 \mathrm{U} / \mathrm{ml}$

Reagent: EDTA $0.1 \mathrm{~mol} / 1$

Standard: G-6-P $2 \mathrm{mmol} / 1$

Reaction mixture (final concentrations):

Buffer $1 \mathrm{ml}$; NADP $10 \mu \mathrm{l}(0.05 \mathrm{mmol} / \mathrm{l})$; EDTA $5 \mu \mathrm{l}(0.5 \mathrm{mmol} / 1)$

\section{Procedure:}

$1 \mathrm{ml}$ of reaction mixture was added to $20 \mu \mathrm{l}$ of standard. E1 was then read. $10 \mu \mathrm{l}$ of G6P-DH $(0.07 \mathrm{U} / \mathrm{ml})$ was then added and the reaction followed to completion (3-5 $\mathrm{min}$ ). E2 was then read. The concentration of standard was calculated using the formula (1). 


\section{4) Lactate}

\section{Principle:}

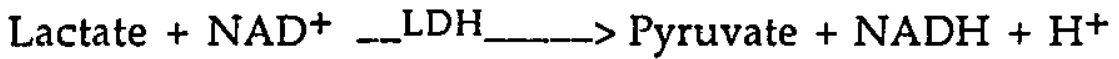

\section{Reagents:}

Buffer: Hydrazine $1.1 \mathrm{~mol} / \mathrm{l}$, pH 9.0 with $1 \mathrm{mmol} / \mathrm{l}$ EDTA

Cofactor: NAD $50 \mathrm{mmol} / \mathrm{l}$

Enzymes: LDH $5500 \mathrm{U} / \mathrm{ml}$ (undiluted)

Standard: Lactate $2 \mathrm{mmol} / 1$

Reaction mixture (final concentrations):

Buffer $1 \mathrm{ml}$; NAD $40 \mu \mathrm{l}(2 \mathrm{mmol} / \mathrm{l})$

\section{Procedure:}

$1 \mathrm{ml}$ of reaction mixture was added to $20 \mu \mathrm{l}$ of standard. E1 was then read. $15 \mu \mathrm{l}$ of $\mathrm{LDH}(82.5 \mathrm{U} / \mathrm{ml})$ was then added and the reaction followed to completion (15-20 min). E2 was then read. The concentration of standard was calculated using the formula (1). 


\section{5/ Phosphocreatine}

Principle:

$\mathrm{PCr}+$ ADP __CK____> Creatine + ATP

ATP + Glucose __HK____ A ADP + G6P

G6P + NADP _ G6P-DH___ 6-P-Gluconolactone + NADPH + $\mathrm{H}^{+}$

\section{Reagents:}

Buffer: TRIS- $\mathrm{HCl} 50 \mathrm{mmol} / \mathrm{l}, \mathrm{BSA} 0.02 \%, \mathrm{pH} 8.1$

Cofactor: NADP $50 \mathrm{mmol} / \mathrm{l}$

Enzymes: CK $380 \mathrm{U} / \mathrm{ml}$; HK $28 \mathrm{U} / \mathrm{ml}$; G6P-DH $7 \mathrm{U} / \mathrm{ml}$

Reagents: ADP $50 \mathrm{mmol} / \mathrm{l}$; Glucose $10 \mathrm{mmol} / \mathrm{l} ; \mathrm{MgCl}_{2} 0.1 \mathrm{~mol} / 1$

Standard: PCr $2 \mathrm{mmol} / \mathrm{l}$

\section{Reaction mixture (final concentrations):}

Buffer $1 \mathrm{ml}$; NADP $50 \mu \mathrm{l}(2.5 \mathrm{mmol} / \mathrm{l}) ; \mathrm{ADP} 10 \mu \mathrm{l}(0.5 \mathrm{mmol} / \mathrm{l}) ; \mathrm{MgCl}_{2} 50 \mu \mathrm{l}$ $(5 \mathrm{mmol} / \mathrm{l}) ;$ Glucose $10 \mu \mathrm{l}(1 \mathrm{mmol} / \mathrm{l}) ; \mathrm{G} 6 \mathrm{P}-\mathrm{DH} 10 \mu \mathrm{l}(0.07 \mathrm{U} / \mathrm{ml}) ; \mathrm{HK} 10 \mu \mathrm{l}$ $(0.28 \mathrm{U} / \mathrm{ml})$

\section{Procedure:}

$1 \mathrm{ml}$ of reaction mixture was added to $20 \mu \mathrm{l}$ of $2 \mathrm{mmol} / \mathrm{l}$ ATP standard. E1 was then read. $10 \mu \mathrm{l}$ of $\mathrm{CK}$ was then added and the reaction followed to completion (10 min). E2 was then read. A further $10 \mu \mathrm{l}$ of $\mathrm{CK}$ was then added to determine the absorbance of the enzyme $\left(E_{C K}\right)$. The concentration of standard was calculated using the formula (1), where: $E=E_{2}-E_{1}-E_{C K}$ 


\section{APPENDIX C}

\section{SINGLE MUSCLE FIBRE GLYCOGEN ASSAY}

\section{Principle:}

$$
\begin{aligned}
& \mathrm{ATP}+\text { Glucose _-HK__ } \longrightarrow \mathrm{G}-6-\mathrm{P}+\mathrm{ADP} \\
& \mathrm{NADP}+\mathrm{G}-6-\mathrm{P} \_\mathrm{G} 6 \mathrm{P}-\mathrm{DH} \_\longrightarrow \text { _ } \\
&
\end{aligned}
$$

\section{$\underline{\text { Reagents: }}$}

Buffer: Tethanolamine-Hydrochloride (TEA-HCl), $400 \mathrm{mmol} / \mathrm{l}$, $\mathrm{pH} 7.8$

Cofactors: NADP $10 \mathrm{mmol} / \mathrm{l} ; \mathrm{MgCl}_{2} 100 \mathrm{mmol} / \mathrm{l}$; EDTA $20 \mathrm{mmol} / \mathrm{l}$; DTT $50 \mathrm{mmol} / \mathrm{l}$; ATP $32 \mathrm{mmol} / \mathrm{l}$; BSA in $\mathrm{NaHCO}_{3}(1 \%$ solution)

Enzymes: Hexokinase 11.2 U/ml; G6P-DH $350 \mathrm{U} / \mathrm{ml}$

Standard: Glucose $20 \mathrm{mmol} / 1$ (stock solution) The working standard $(100 \mu \mathrm{mol} / \mathrm{l})$ was prepared daily from the stock solution as follows: $50 \mu \mathrm{l}$ of stock solution into 10 $\mathrm{ml}$ of double-distilled water.

Immediately prior to the analysis sufficient reaction mixture for blanks, standards, control and samples was prepared as follows:

\section{Reaction mixture:}

Buffer $1 \mathrm{ml} ; \mathrm{MgCl}_{2} 160 \mu \mathrm{l}$; EDTA $100 \mu \mathrm{l}$; DTT $40 \mu \mathrm{l}$; NADP $200 \mu \mathrm{l}$; ATP 144 $\mu \mathrm{l} ; \mathrm{BSA}, \mathrm{NaHCO}_{3} 160 \mu \mathrm{l} ; \mathrm{H}_{2} \mathrm{O} 250 \mu \mathrm{l} ; \mathrm{G} 6 \mathrm{P}-\mathrm{DH} 0.32 \mu \mathrm{l}$ 


\section{Procedure:}

i/ Reaction mixture $(0.6 \mathrm{ml})$ was added to cuvettes containing $50 \mu \mathrm{l}$ of double-distilled water (blank), standard, control and samples.

ii/ The cuvettes were mixed and incubated for $15 \mathrm{~min}$.

iii/ Fluorescence was then read.

iv/ Following this, $10 \mu \mathrm{l}$ of hexokinase was added to each cuvette before they were mixed and incubated for $15 \mathrm{~min}$.

v/ Fluorescence was then read.

vi/ Where enough sample was left the above procedure was repeated (duplicate analysis). 
i/ A $5 \mathrm{mmol} / \mathrm{l} \mathrm{NADH}$ stock solution was prepared

ii/ Six NADH standards were then prepared from the stock solution as follows:

Stock solution $(\mu \underline{1})$

0

10

25

50

75

100
Water (ml)

2.5

2.5

2.5

2.5

2.5

2.5 $\sim \mathrm{NADH}] \mu \mathrm{mol} / \mathrm{l}$

0

20

50

100

150

200

iii/ The absorbance of the NADH standards was then measured against the absorbance of water (blank) on a spectrophotometre at $340 \mathrm{~nm}$.

iv/ The exact concentration of the NADH standards (mmol/l) was then calculated using the formula:

\section{Absorbance of standard - Absorbance of blank / 6.22}

where: 6.22 is the coefficient of extinction of NADH at $340 \mathrm{~nm}$.

v/ Following this, $50 \mu \mathrm{l}$ of each NADH standard was pipetted into fluorometric cuvettes. Double-distilled water $(0.6 \mathrm{ml})$ was then added to each cuvette. The cuvettes were mixed and their fluoresence was read.

$\mathrm{vi} /$ The fluoresence of the standards was then plotted against the product of the concentrations of the NADH standards and the dilution factor (i.e. $50 / 650$ ). The resulting regression equation enabled the calculation of the slope $\mathrm{K}$, which related the values detrmined by the fluorimeter to the values accurately determined by the spectrophotometer. 
Determination of glucose concentration in each cuvette

The concentration of glucose in each cuvette $(Z)$ was calculated using the formula:

$$
Z=\left\{\left(V_{2} * Y_{2}-V_{1}^{*} Y_{1}\right)-\left(V_{2} * X_{2}-V_{1} * X_{1}\right)\right\} / V_{2} * K
$$

where: $\quad V_{1}=$ volume in cuvette without hexokinase added

$\mathrm{V}_{2}=$ volume in cuvette with hexokinase added

$\mathrm{X}_{1}=$ Fluorescence of blank before hexokinase added

$\mathrm{X}_{2}=$ Fluorescence of blank after hexokinase added

$Y_{1}=$ Fluorescence of standard, control or sample before hexokinase added

$Y_{2}=$ Fluorescence of standard, control or sample after hexokinase added 
Determination of glycogen concentration (G) from glucose concentration in each cuvette

The glycogen concentration (G) of each sample was calculated using the formula:

$$
G=\{(Z a-Z b-Z c) * E F * V / W) * D
$$

where: $\quad \mathrm{Za}=$ concentration of glucose in cuvette containing sample with the enzyme amyloglucosidase $\mathrm{Zb}=$ concentration of glucose in cuvette containing the sample without the enzyme amyloglucosidase

$\mathrm{Zc}=$ concentration of glucose in cuvette with control sample

$\mathrm{EF}=$ Dilution factor in extraction and degradation

$\mathrm{V}=$ Volume of $\mathrm{KOH}$ in which pooled fibres were extracted

$\mathrm{W}=$ weight of pooled fibres $(\mu \mathrm{g})$

$\mathrm{D}=$ Dilution factor in glucose assay (13.2) 
